\title{
RISK-BASED APPROACH IN HighWAY GeOMETRIC DESIGN
}

\author{
By \\ Mohamed Sarhan \\ B.Sc., Ain Shams University \\ M.A.Sc., Carleton University
}

A thesis submitted to the Faculty of Graduate Studies and Research in the partial fulfilment of the requirements of the degree of

Doctor of Philosophy

In Civil Engineering

Department of Civil and Environmental Engineering Carleton University

Ottawa, Ontario, Canada

(C) copyright

2008, Mohamed Sarhan

The Doctor of Philosophy in Civil Engineering is a joint program with the University of Ottawa Administrated by the Ottawa-Carleton Institute for Civil Engineering 


$\begin{array}{ll}\begin{array}{l}\text { Library and } \\ \text { Archives Canada }\end{array} & \begin{array}{l}\text { Bibliothèque et } \\ \text { Archives Canada }\end{array} \\ \begin{array}{l}\text { Published Heritage } \\ \text { Branch }\end{array} & \begin{array}{l}\text { Direction du } \\ \text { Patrimoine de l'édition }\end{array} \\ \begin{array}{l}\text { 395 Wellington Street } \\ \text { Ottawa ON K1A 0N4 } \\ \text { Canada }\end{array} & \begin{array}{l}\text { 395, rue Wellington } \\ \text { Ottawa ON K1A 0N4 } \\ \text { Canada }\end{array}\end{array}$

Your file Votre référence ISBN: 978-0-494-43910-4 Our file Notre référence ISBN: 978-0-494-43910-4

NOTICE:

The author has granted a nonexclusive license allowing Library and Archives Canada to reproduce, publish, archive, preserve, conserve, communicate to the public by telecommunication or on the Internet, loan, distribute and sell theses worldwide, for commercial or noncommercial purposes, in microform, paper, electronic and/or any other formats.

The author retains copyright ownership and moral rights in this thesis. Neither the thesis nor substantial extracts from it may be printed or otherwise reproduced without the author's permission.
AVIS:

L'auteur a accordé une licence non exclusive permettant à la Bibliothèque et Archives Canada de reproduire, publier, archiver, sauvegarder, conserver, transmettre au public par télécommunication ou par l'Internet, prêter, distribuer et vendre des thèses partout dans le monde, à des fins commerciales ou autres, sur support microforme, papier, électronique et/ou autres formats.

L'auteur conserve la propriété du droit d'auteur et des droits moraux qui protège cette thèse. $\mathrm{Ni}$ la thèse ni des extraits substantiels de celle-ci ne doivent être imprimés ou autrement reproduits sans son autorisation.
In compliance with the Canadian Privacy Act some supporting forms may have been removed from this thesis.

While these forms may be included in the document page count, their removal does not represent any loss of content from the thesis.
Conformément à la loi canadienne sur la protection de la vie privée, quelques formulaires secondaires ont été enlevés de cette thèse.

Bien que ces formulaires aient inclus dans la pagination, il n'y aura aucun contenu manquant.

\section{Canada}




\section{Abstract}

In current geometric design guides, the highway elements are designed using the deterministic approach. This approach considers below average driver characteristics and vehicle capabilities to produce designs that would satisfy the majority of road users. However, a design following this approach could be conservative and uneconomic since the probability of having simultaneous critical values for all design parameters could be very low. In addition, the current design methods rely on either horizontal or vertical projection of the road alignment, which may cause either underestimation or overestimation of the road supply.

In this research, a comprehensive approach for geometric design was developed based on reliability theory. The approach was applied to the sight distance design basis using the required sight distance (RqSD) as a demand and the distance actually available to drivers (AvSD) as a supply. Both simulation and analytical reliability techniques were used in the analysis. For RqSD calculations, a new approach was developed to account for the continuous change of longitudinal grade, expected loss of friction on horizontal curves, and it considers both deceleration rate and friction coefficient. As for AvSD calculations, both two and three dimensional values were calculated taking advantage of two existing programs based on the finite element technique.

Using Visual Basic.Net programming language, a computer program was developed; namely sight distance evaluation system (SDES). Using SDES, profiles can be generated for RqSD and/or AvSD at pre-determined intervals. At a given road section, 
RqSD and AvSD distributions can be evaluated to determine the probability of hazard $(\mathrm{POH})$, which reflects the percentage of drivers who may suffer insufficient sight distance. $\mathrm{POH}$ profile was further evaluated in a real case study using data from Highway 61, Ontario. The $\mathrm{POH}$ values less than $0.05 \%$ have mainly resulted along the entire route except for a few sections that had a maximum $\mathrm{POH}$ of $7.45 \%$. In addition, a reliabilitybased approach was developed to locate lateral obstructions along three-dimensional alignments using a certain $\mathrm{POH}$. Two design aids were further developed for design and evaluation purposes on 400-m curves and 2H:1V side slope. 


\section{Acknowledgments}

The author would like to express his deep appreciation and grateful thanks to Dr. Yasser Hassan for his invaluable time, endless assistance, and continual guidance in supervising this thesis. My sincere gratitude also goes to Dr. A. O. Abd El Halim, Carleton University, for his support and encouragement and Dr. J. J. Salinas, Carleton University, for his help and guidance. Additional gratitude is extended to all my professors, fellow colleagues, and friends for their help.

Financial support by Carleton University and the Natural Sciences and Engineering Research Council (NSERC) is gratefully acknowledged.

Also, I would like to express my deepest gratitude to my parents and all family members whom for their prayer and wishes I was able to deliver this research. Finally, special thanks and appreciation are due to my beloved wife Amal and my two little daughters Nour and Jana whom their love, understanding, and encouragement have made this research successful. 


\section{Table of Contents}

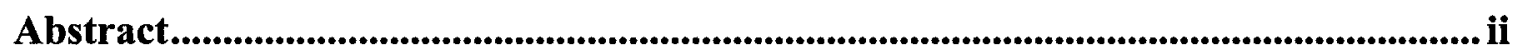

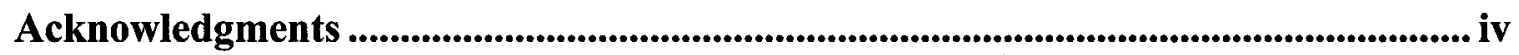

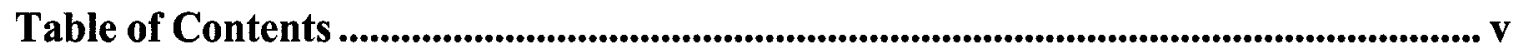

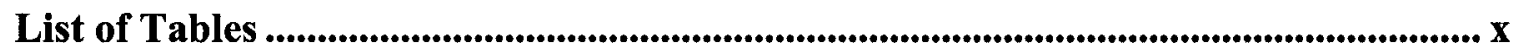

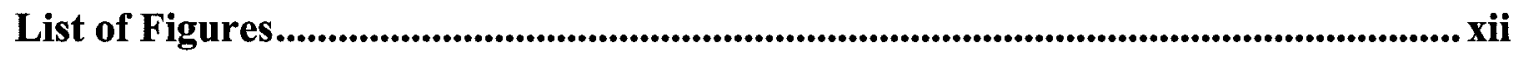

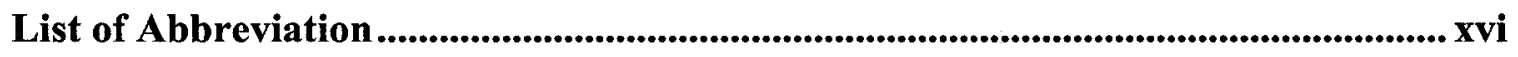

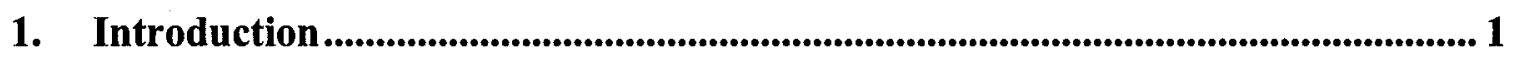

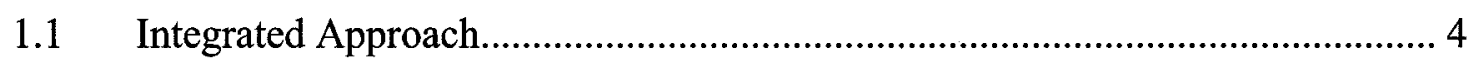

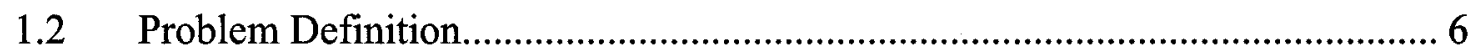

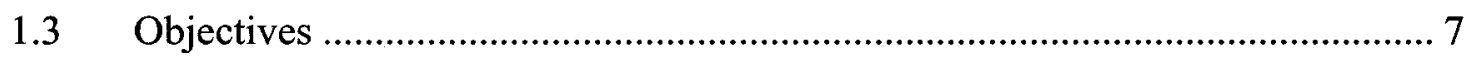

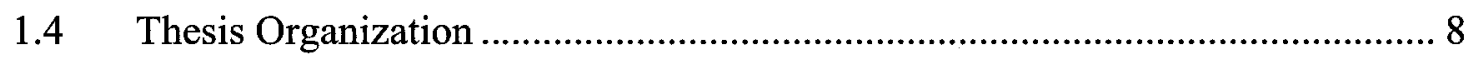

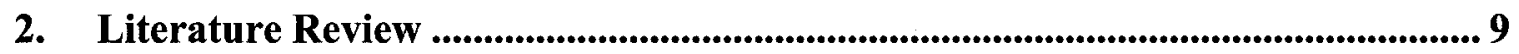

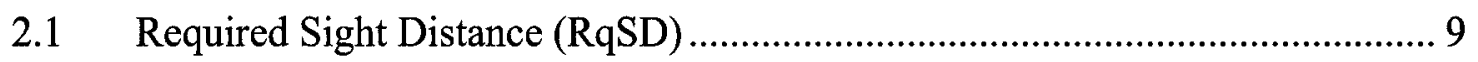

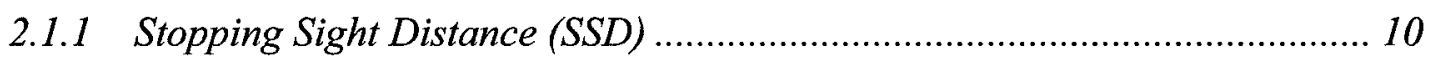

2.1.2 Decision Sight Distance (DSD) ....................................................... 13

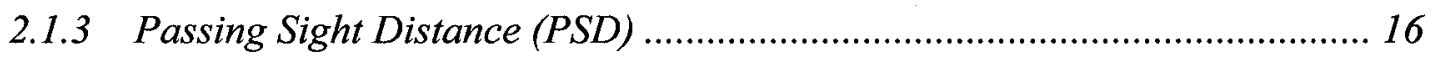

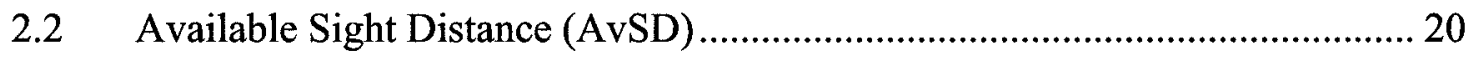

2.2.1 Sight Distance Based on Horizontal Alignment..................................... 21

2.2.2 Sight Distance Based on Vertical Alignment ....................................... 24

2.2.3 Sight Distance Based on Combined Alignment ...................................... 28

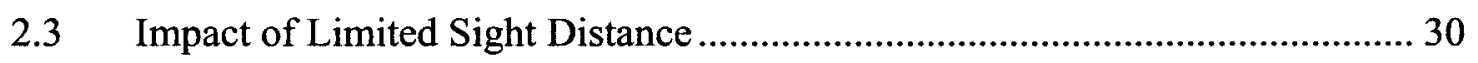

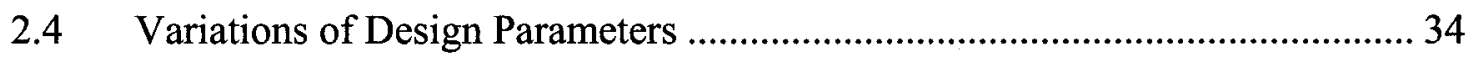




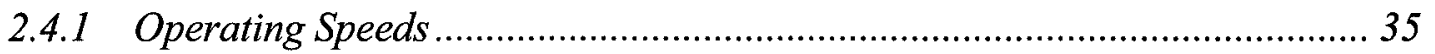

2.4.2 Acceleration and Deceleration Rate ……………...................................... 37

2.4.3 Coefficient of Pavement Friction............................................................... 40

2.4 .4 Perception-Reaction Time …………………........................................ 41

2.4.5 Driver Eye, Object, and Headlight Height …………………………....... 45

2.5 Risk Assessment and Reliability Analysis in Transportation ............................. 48

3. Reliability-Based Design Approach........................................................................57

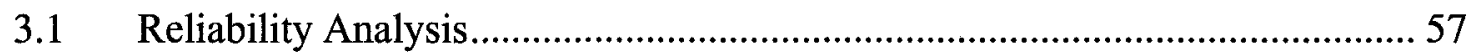

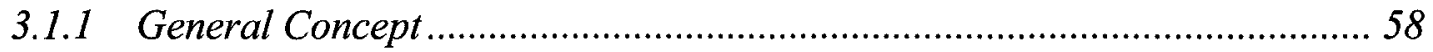

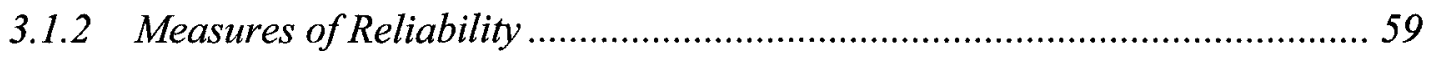

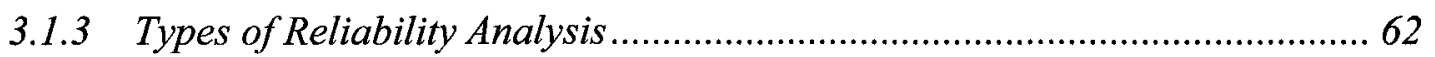

3.1.4 Reliability Analysis in Geometric Design ................................................. 65

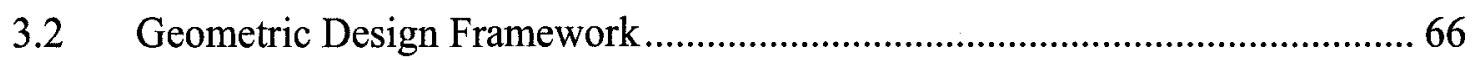

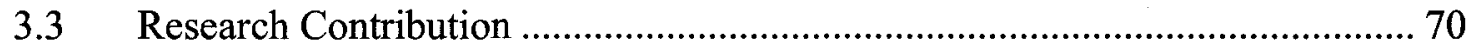

3.4 Sight Distance Evaluation System "SDES"....................................................... 71

3.4.1 SDES Framework - Pre-Analysis .......................................................... 72

3.4.1.a Generate alignment and obstructions..................................................... 73

3.4.1.b Design parameters identification and other settings .............................. 75

3.4.1.c Options for design flexibility ............................................................... 76

3.4.2 SDES Framework - Sight Distance Assessment ………………………...... 77

3.4.2.a Variables generator ................................................................................ 77

3.4.2.b Required sight distance .......................................................................... 79

3.4.2.c Road surface creation and available sight distance................................. 80 


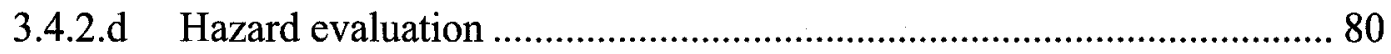

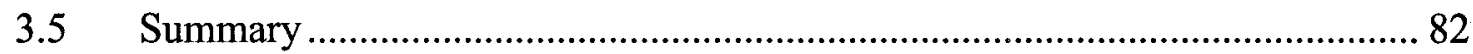

4. Probabilistic Required Sight Distance …......................................................................... 83

4.1 Approximations in Current Practice ........................................................... 83

4.1.1 Consideration of Longitudinal Grade ....................................................... 84

4.1.2 Influence of horizontal Curvature on Longitudinal Friction ........................ 86

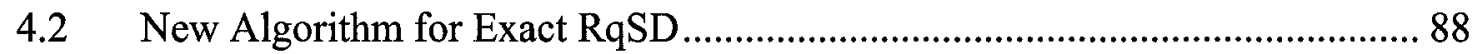

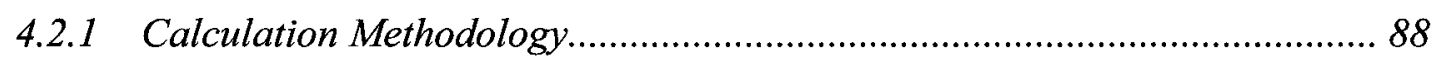

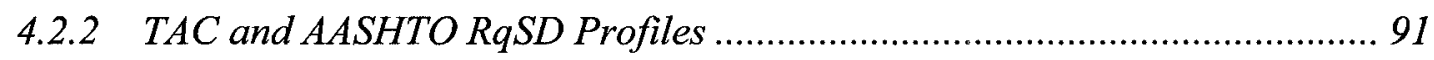

4.3 Probabilistic versus Deterministic Approaches ............................................. 93

4.3.1 Probabilistic Approach using Simulation Reliability Technique.................. 93

4.3.2 Probabilistic Approach using Analytical Reliability Technique .................. 98

4.4 RqSD Considering both Deceleration and Friction ......................................... 99

4.5 Field Measurement of Friction Coefficient............................................... 102

4.5.1 T-10 Friction Trailer ...................................................................... 103

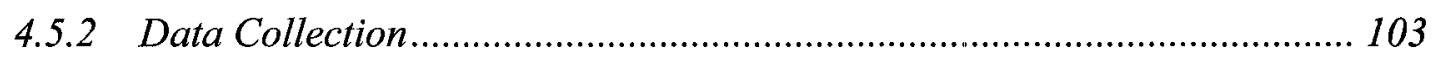

4.5.3 Descriptive Analysis and Findings ....................................................... 107

4.5.4 Relationship between Speed and Friction............................................... 110

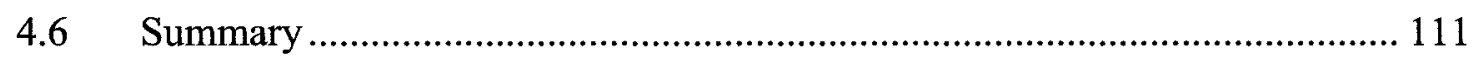

5. Probabilistic Available Sight Distance .................................................................... 112

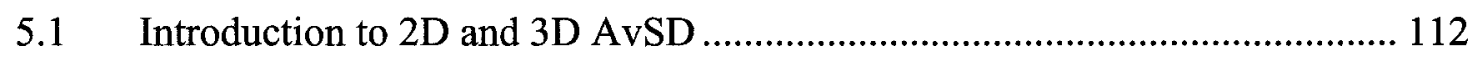

5.2 SDES Simulation and Precision............................................................... 116

5.2.1 AvSD on Vertical-Only Alignment ......................................................... 116 
$5.2 .2 A v S D$ on Horizontal-Only Alignment........................................... 118

5.3 Probabilistic versus Deterministic AvSD ............................................... 120

5.3.1 2D-V AvSD: Simulation Method ......................................................... 121

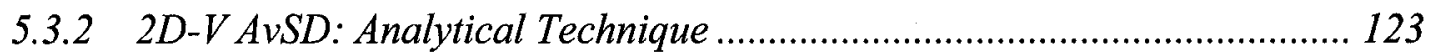

5.3.3 2D-H AvSD: Simulation Method ......................................................... 124

5.3.4 2D-H AvSD: Analytical Technique .................................................. 126

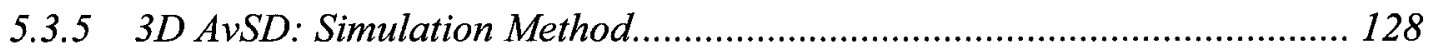

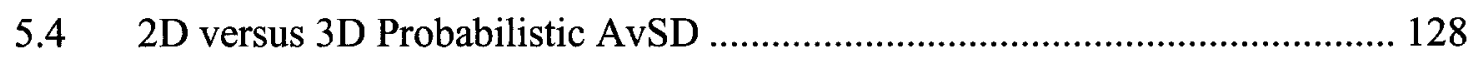

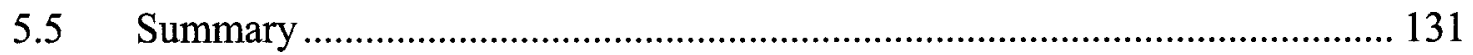

6. Hazard Assessment and Case Study................................................................... 133

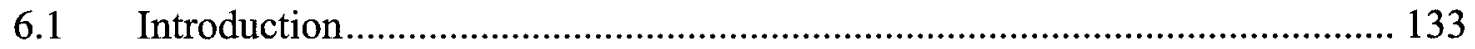

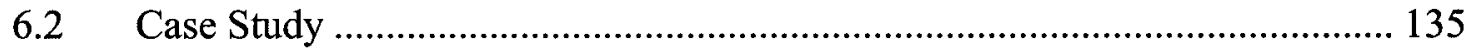

$6.2 .1 \quad$ Road Segment Description...................................................... 135

6.2.2 Speed Estimation on Two-Lane Roadways ........................................... 139

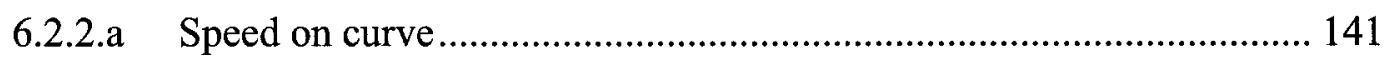

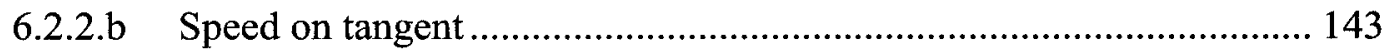

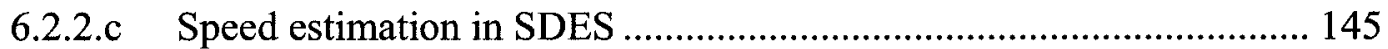

6.2.3 Road Surface Condition on Highway 61 ........................................... 146

6.2.4 Sight Distance and POH Profiles .................................................. 150

6.2.4.a Number of simulations........................................................... 150

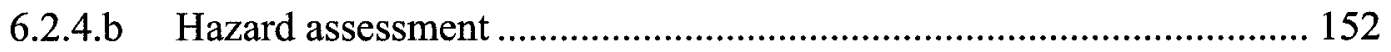

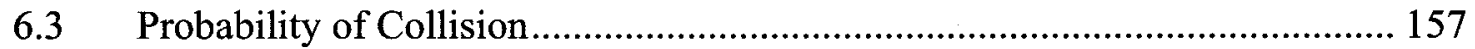

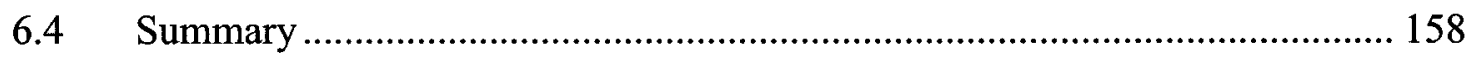


7. Contribution to Design Practice ........................................................................................... 159

7.1 Current Design Methodology .................................................................. 159

7.2 Critique of Current Methodology ……………............................................. 161

7.3 New 3D Reliability-Based Design Methodology …………………………..... 164

7.4 Establishment of a Hazard-Based Design Aid................................................ 167

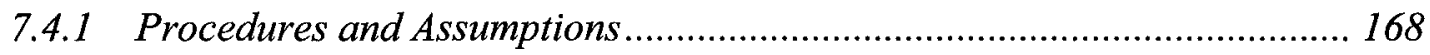

7.4.2 Design Aid and Application ................................................................. 171

7.5 Hazard Evaluation of an Existing Design ..................................................... 174

7.6 Interrelationship between Speed and AvSD ................................................ 177

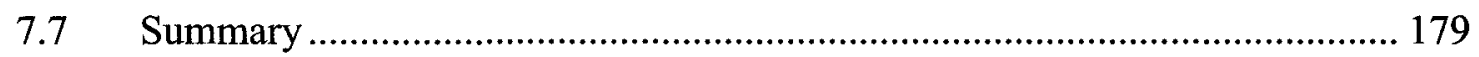

8. Conclusions and Recommendations ................................................................................... 180

8.1 Summary and Conclusions .................................................................... 180

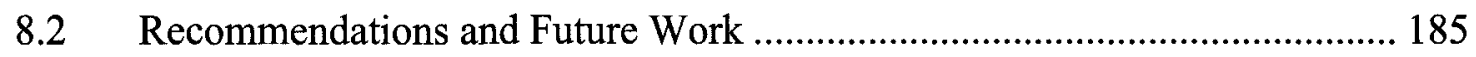

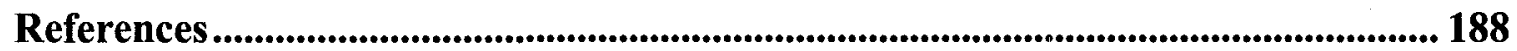

Additional Bibliography ............................................................................................................ 196 


\section{List of Tables}

Table 2-1: Mean Speed Reductions Observed in Different Locations in Washington (WA), Taxes (TX), and Illinois (IL) (Adapted from Fambro et al. 2000a).

Table 2-2: Summary of Constant Deceleration Rates for Different Pavement Conditions and Braking Systems (Adapted from Fambro et al. 2000c). 38

Table 2-3: Summary of Driver Deceleration to an Unexpected Object (Adapted from Fambro et al. 2000c).

Table 2-4: Summary of Driver Deceleration to an Expected Object (Adapted from Fambro et al. 2000c). 39

Table 2-5: Coefficient of Friction for Wet Pavements (Adapted from TAC 1999). 40

Table 2-6: Summary of Surprise and Anticipated Perception-Brake Reaction Time

Studies (Adapted from Fambro et al. 1998).

Table 2-7: Summary of Perception-Brake Response Times (Adapted from Fambro et al. 1998).

Table 2-8: Summary of Driver Eye Height Studies (Adapted from Fitzpatrick et al. 1998).

Table 2-9: Driver Eye, Headlight, Taillight, and Vehicle Heights for Different Vehicle Types (Adapted from Fitzpatrick et al. 1998). 48

Table 4-1: RqSD on Horizontal Curves Considering Reduced Friction (Adapted from

TAC 1999). 88

Table 4-2: Mean and Standard Deviation of Design Parameters. 94

Table 4-3: Locations and Dates for Data Collection. 105

Table 4-4: Descriptive Analysis of Speed and Friction. 108 
Table 5-1: Summary of $h_{1}$ and $h_{2}$ (Deterministic and Probabilistic).

Table 5-2: Comparison between 2D and 3D design Approaches at Station 400........... 130

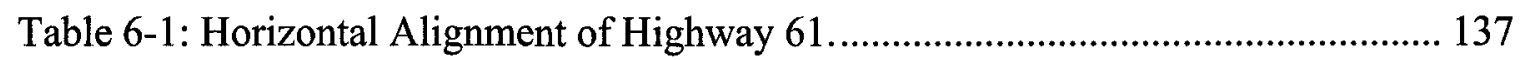

Table 6-2: Vertical Alignment of Highway 61............................................................. 138

Table 6-3: Curve and Speed Database Used in Estimating Speed Distribution. ............. 140

Table 6-4: Model for the Mean Speed on Horizontal Curves.......................................... 142

Table 6-5: Speed on Approach Tangents of the 20 Curve............................................... 144

Table 6-6: Sample of the Weather Condition on Highway 61 for the month of May.... 147

Table 6-7: Summary of Different Weather Conditions during the Year 2002 ............... 148

Table 6-8: Mean Values of Several Meteorological Parameters as Posted in The Weather Network web site. 149

Table 6-9: POH using Different Numbers of Iterations................................................ 152

Table 6-10: Characteristics of the Friction Distribution. ............................................... 153

Table 6-11: Road Sections with POH greater than 1 \%................................................ 157

Table 7-1: Sample Correction of Lateral Clearance on Sharp Curves (Adapted from TAC 1999). 163 


\section{List of Figures}

Figure 1-1: Probability of Hazard Due to Insufficient Supply. ......................................... 5

Figure 2-1: Components of Required PVSD (Hassan and Easa 2000)............................ 15

Figure 2-2: Elements of PSD (Adapted from TAC 1999) …………………………...... 17

Figure 2-3: Required PSD for Passenger Car and Trucks in Comparison with Design

Guides (Harwood and Glennon 1989)................................................................. 18

Figure 2-4: Elements of PSD (Wang and Cartmell 1998) .............................................. 19

Figure 2-5: Determination of Horizontal Sight Distance (Adapted from AASHTO 2004).

Figure 2-6: Check of HLSD on Simple Horizontal Curves; $\alpha=20^{\circ}$ (Easa and Hassan

Figure 2-7: Sight Distance on Crest Vertical Curves (Adapted from AASHTO 2004)... 25

Figure 2-9: AvSD for Application Example (Easa et al. 1996).

Figure 2-10: SSD versus Length of Road for K-177 in Riley Country, Kensus, U.S.

(Nehate and Rys 2006). 30

Figure 2-11: Limited Sight Distance on Road SS 166 (Discetti and Dell'Acqua 2008). . 33

Figure 2-12: Relationship between AvSD and $85^{\text {th }}$ Percentile Speed (Discetti and Dell'Acqua 2008).

Figure 2-13: Relationship between Operating Speeds $\left(\mathrm{V}_{85}\right.$ and $\left.\mathrm{V}_{50}\right)$ and Curve Radius

(Andjus and Maletin 1998). 36

Figure 2-14: Relationship between Standard Deviation and V50 (Andjus and Maletin 1998). 
Figure 2-15: Analysis of Speed Distribution at $50 \mathrm{mph}$ Design Speed (E1 Khoury and Hobeika 2006)

Figure 2-16: The Effect of Age and Sex on the risk of causing a vehicle collision (Claret et al. 2003) 50

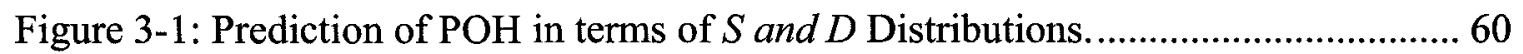

Figure 3-2: Prediction of $\mathrm{POH}$ in terms of a Performance Function. ........................... 61

Figure 3-3: Simplification of Monte Carlo Simulation Technique.................................63

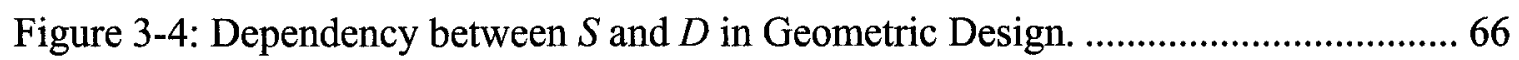

Figure 3-5: Reliability-Based Framework for Highway Geometric Design.................... 67

Figure 3-6: The Outline of the Expected Contribution of this Research. ........................ 70

Figure 3-7: Suggested Framework to Evaluate Sight Distance Sufficiency.................... 73

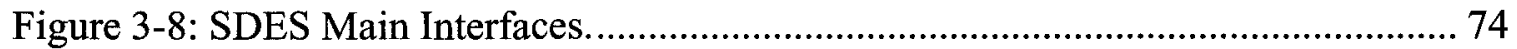

Figure 4-1: Forces Acting on a Vehicle's Tire at Stopping on Horizontal Curves........... 87

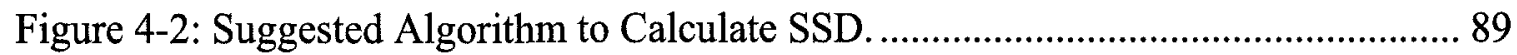

Figure 4-3: Influence of Longitudinal Grade and Horizontal Curvature on TAC RqSD. 92

Figure 4-4: Influence of Longitudinal Grade on AASHTO RqSD............................ 92

Figure 4-5: Deterministic versus Probabilistic AASHTO RqSD (100,000 iterations).... 95

Figure 4-6: Deterministic versus Probabilistic AASHTO RqSD. ................................ 97

Figure 4-7: New Methodology to Calculate the RqSD Distribution using Both

Deceleration and Friction. 102

Figure 4-8: CFME T-10 Trailer for Continuous Friction Measurement....................... 104

Figure 4-9: CFME Computer Water Tank Fitted in the Minivan................................. 104

Figure 4-10: Snow and Dry Surface Conditions during Winter Time.......................... 106 
Figure 4-11: Distributions of Friction Coefficient for Different Driving Scenarios. ..... 108

Figure 4-12: Relationship between Friction and Speed......................................... 111

Figure 5-1: A Simple Cross-Section at the Midpoint of a Horizontal Curve. ............... 113

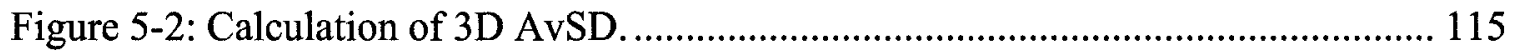

Figure 5-3: Assumed Cross-sections and Progress of Superelevation........................... 117

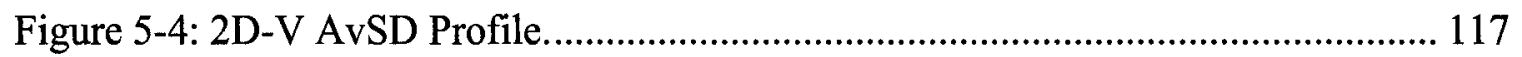

Figure 5-5: 2D versus 3D Approaches for AvSD on flat horizontal curves.................. 119

Figure 5-6: Difference between 2D and 3D AvSD on Horizontal-Only Curves........... 120

Figure 5-7: TAC 2D-V AvSD profile versus Probabilistic Approach........................... 122

Figure 5-8: Deterministic versus Probabilistic 2D-V AvSD at Station 400.................. 122

Figure 5-9: Profile and Distribution of 2D-H AvSD at Station 400........................... 125

Figure 5-10: Influence of Side Slope on AvSD Variation......................................... 127

Figure 5-11: TAC Deterministic versus Probabilistic Approaches for 3D AvSD........ 129

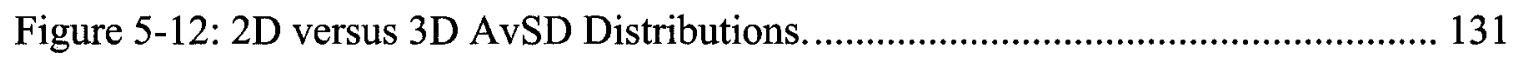

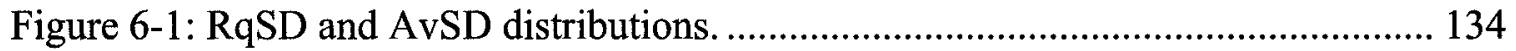

Figure 6-2: Three-Dimensional Schematic of Highway 61 Segment Used in the Analysis.

Figure 6-3: Curve Radius versus Mean Speed on Curves' Mid-Points......................... 142

Figure 6-4: Curve Radius versus Standard Deviation of Speed on Curves' Mid-Points. 143

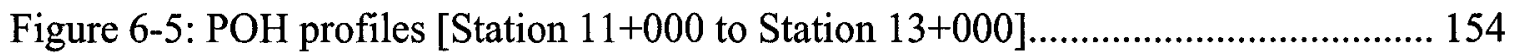

Figure 7-1: Determination of Lateral Clearance using Current Method (Adapted from

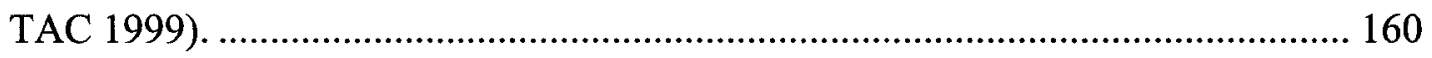

Figure 7-2: Different Cross-sections at the Mid-Point of AvSD. ............................... 162 
Figure 7-3: A Layout of the Proposed Design Methodology. ........................................ 165

Figure 7-4: Reliability-Based Method for 3D-HSO Determination at $R=400 \mathrm{~m} . \ldots \ldots \ldots . . .172$

Figure 7-5: Hazard Evaluation of 3D Alignment at $R=400 \mathrm{~m}$ and $\mathrm{SS}=2 \mathrm{H}: 1 \mathrm{~V} . \ldots \ldots \ldots . .176$

Figure 7-6: Influence of AvSD on Speed (Discetti and Dell'Acqua 2008 and Andjus and

Maletin 1998) 


\section{List of Abbreviation}

$\begin{array}{ll}\text { AASHTO } & \text { American Association of state Highway and Transportation Officials } \\ \text { AvSD } & \text { Available Sight Distance } \\ \text { CF } & \text { Central Factor of Safety } \\ \text { D } & \text { Demand } \\ \text { DSD } & \text { Decision Sight Distance } \\ \text { CF } & \text { Central Factor of Safety } \\ \text { F } & \text { Factor of Safety } \\ \text { HLSD } & \text { Headlight Sight Distance } \\ \text { HSM } & \text { Highway Safety Manual } \\ \text { M } & \text { Probability of Hazard } \\ \text { POH } & \text { Required Sight Distance } \\ \text { PUTCD } & \text { Manual on Uniform Traffic Control Devices } \\ \text { PR } & \text { Perception and Reaction } \\ \text { PSD } & \text { Passing Sight Distance } \\ & \end{array}$


Sight Distance

SSD

Stopping Sight Distance

TAC

Transportation Association of Canada

2D-H

Two Dimensional Horizontal

2D-V

Two Dimensional Vertical 


\section{CHAPTER 1}

\section{INTRODUCTION}

There has been an ongoing recognition of the role that reliability theory can play in different engineering fields to provide more realistic design approaches. However, more effort can still be devoted to enhance current methods of highway geometric design. Thousands of years back, roads have been used for both civil and military purposes firstly by the Romans who recognized the essential needs to move quickly from one location to another. The word highway even has its origin since the Roman era, when roads were elevated by almost one meter above the land level. At that time, roads were structurally designed to simply last for a long time using four layers upon which broken stones were placed to work as a durable rolling surface. However, a need for geometric design was not recognized at that point of time. Way later, and in early 1900 s as stated by Hall and Turner (1998), roads in the U.S. were designed geometrically to carry animal-powered vehicles based on simple criteria such that curve radii and grades should help provide easy navigation of these vehicles at low speeds.

In the early 1930s, the concept of balanced design was first proposed so that drivers do not have to slow down to safely traverse a curved section (Merritt 1988). Theoretically, the centripetal force, which is the primary cause of vehicle instability on horizontal curves, has to be balanced to satisfy acceptable driving comfort and, more 
importantly, to meet safety requirements. The geometric design proceeded after that to include the design of vertical curves in order to satisfy enough sight distance (SD) for road users. The concept that was used to design vertical curves considered minimum curve lengths that would enable drivers to stop or pass safely. Sag vertical curves are further checked against driving comfort since all forces that are acting on a moving vehicle are working downward in the same direction.

In the early 1970 s, the geometric design moved from individual curve design to include design consistency along an entire alignment. The concept aims at harmonizing operational conditions over successive curves and tangents. Several consistency measurements were developed such as operating speed, driver workloads, and alignment indices including average radius, ratio between maximum and minimum radii, average rate of vertical curvature, and ratio between individual curve radius and average radius. All these methods were to help enhance the operational conditions and protect drivers from sudden and unexpected changes in road design.

In the late 1980s, highway geometric design took further steps to include attempts at enhancing safety performance on road sections and explicitly linking it to the design decisions. This was done through a five-year series of sessions held by the Transportation Research Board (TRB) committees on Geometric Design and the Operational Effects of Geometric Design (Reagan 1994). Repeated collisions and deterioration of safety performance were behind seeking design methodologies that would protect road users during their daily trips. In 1999, eight TRB committees suggested and sponsored a safety authoritative document, named later as Highway Safety Manual (HSM). The main target 
of this manual is to "provide the best factual information and tools available, in a useful form, to facilitate roadway planning, operations, and maintenance decisions based upon explicit consideration of their safety consequences" (HSM 2007). An interim document containing the highway safety materials is expected to be available in the summer of 2009 (HSM 2007).

In the late 1990s, the effect of combining horizontal and vertical curves was noticed to influence the perception of such combinations. Subsequently, geometric design was encouraged to involve the three dimensional (3D) design approach rather than the traditional two dimensional (2D) one (Hassan et al. 1996; Hassan and Easa 1998a; and Hassan 2004). It was repeatedly emphasized that horizontal curves would look sharper when combined with crest vertical curves whereas they would seem flatter if combined with sag vertical curves. This would probably cause drivers to adapt different speeds than those expected in the design stage if the traditional 2D approaches are used. The use of 2D approaches could also underestimate or overestimate the road supply and hence a design could become hazardous or uneconomic.

Moreover, to improve the reliability of transportation systems, the inclusion of the probabilistic approach in a transportation context was recently highlighted in the literature. As will be addressed in more detail later in this thesis, the probabilistic approach depends mainly on the probability/reliability analysis to determine possibilities of whether a supply would succeed or fail to meet demands. The consideration of reliability analysis in transportation was initiated by Ben-Akiva (1985) and continued in 
the 1990s to include design of different road elements (Easa 1994; Navin and Zengh 1998; Easa 2000; Easa et al. 2004; E1 Khoury and Hobeika 2006).

\subsection{Integrated Approach}

The work proposed in this thesis puts all these ideas together and produces a suggested geometric design framework. This framework is a comprehensive design methodology that takes into account the 3D design approach, highway safety, and it relies mainly on reliability in the geometric design process. On one hand, the framework considers the $3 \mathrm{D}$ approach in the geometric design rather than the traditional $2 \mathrm{D}$ approach. Obviously, the use of either a horizontal or vertical projection of a highway alignment ignores the effect that the third dimension may have in the geometric design. The 2D approach may therefore result in a highway feature (e.g., a horizontal curve radius) that underestimates or overestimates drivers' need. Subsequently, such a highway feature may become hazardous for road users. The $2 \mathrm{D}$ approach may also cause erroneous perception of road features by road users. Failing to perceive actual design criteria by road users could lead to unfavourable driving behaviours and hence potential of collisions. Therefore, road designers are responsible for providing a design that agrees with the drivers' perceptions and expectations.

In addition, the deterministic approach in current geometric design guides is used widely ignoring the variation within a specific population. Such variation encourages the use of the probabilistic approach to satisfy the disparity of drivers' responses, vehicles' capabilities, and environmental conditions in the design of a specific road feature. Figure 1-1 shows briefly that the road design, driver characteristics, vehicle capabilities, and 
prevailing weather conditions may cause several design parameters to vary such as operating speed and acceleration/deceleration rate. The diagram continues to show a range of demand requirements that drivers may need as a result of the variation in such design parameters. This range stands against a range of supply that is normally provided to drivers. The demand and supply could describe design elements such as sight distance or friction coefficient. Using several reliability techniques, the two distributions can be evaluated to reveal the anticipated hazard due to a certain design.

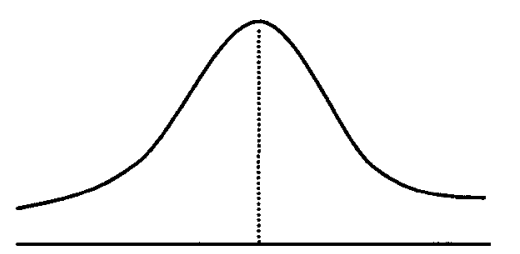

Parameters' Variation

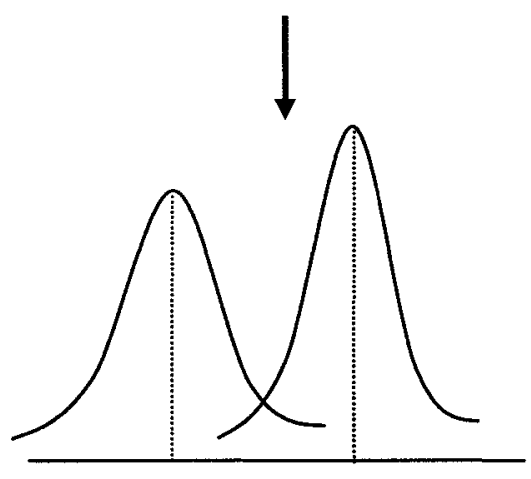

Demand

(D)

(S)

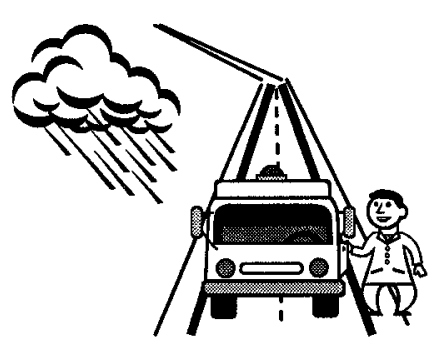

Road, Vehicle, Environment, and Driver

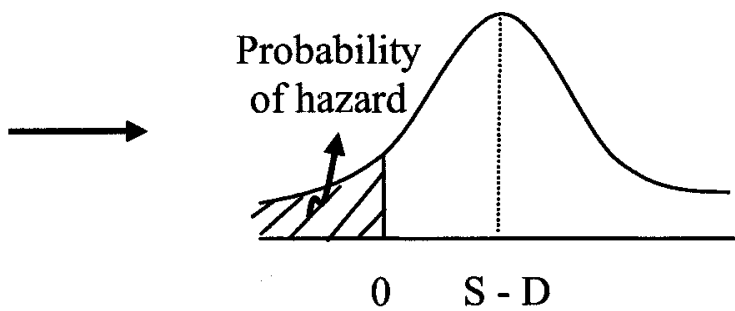

Figure 1-1: Probability of Hazard Due to Insufficient Supply. 


\subsection{Problem Definition}

Reliability analysis has been applied to several design areas of civil engineering. However, and based on relevant research, there is an obvious lack of applying the probabilistic approach in the highway geometric design. The deterministic approach being used in current design guides such as the American Association of State Highway and Transportation Officials (AASHTO) and the Transportation Association of Canada (TAC) has rigid design criteria and hence does not consider the existence of the variation among all contributing factors. The deterministic concept might therefore be questionable since the consequences of violating these design criteria are yet to be identified.

For example, and using the traditional deterministic design approach, a design of a crest vertical curve starts from a design speed that is used to calculate a single value of the stopping sight distance (SSD). Assuming that this value should be equal to or less than the supplied SD, a minimum length of this crest vertical curve is mathematically calculated using the $2 \mathrm{D}$ projected layout of this curve. This design approach can be refuted for more than one reason. First, and because of the uncertainty of several design parameters such as speed, perception and reaction time, and friction coefficient, the use of a single value of SSD is insufficient to describe the population. Second, the determination of the curve length using this $2 \mathrm{D}$ design approach neglects the fact that drivers perceive road features in the $3 \mathrm{D}$ environment. Therefore, the supplied SD that is believed to be available to drivers at a specific point using this traditional approach is not the actual one that drivers can see ahead from the same point. 
Third and most importantly, if the SSD on a specific highway section could not be satisfied, the tradeoffs between costs and safety performance are not explicitly recognized. Practically, there are no physical limits after which safety performances suddenly drop. Therefore, it becomes more appropriate to evaluate the expected gradual changes of safety performances when available sight distances changes. Using the reliability theory, current design criteria can be expanded to include different risk levels. In other words, a probabilistic design approach can help evaluate designs on a scale of appropriateness instead of the misleading notion of right and wrong design.

\subsection{Objectives}

Based on the previous discussion, the primary objectives of this research are as follows:

1. To highlight the weaknesses of the current deterministic design approach of highway geometric design.

2. To develop a new geometric design methodology based on the reliability analysis.

3. To show the application of the developed methodology and use it to highlight the difference between the deterministic and probabilistic approaches.

4. To establish a reliability-based methodology for risk assessment and show its application on a case study.

5. To develop a friendly-interface computer program based on the probabilistic approach for risk evaluation of both new and existing designs. 


\subsection{Thesis Organization}

In CHAPTER 2, a literature review will present several previous studies related to required and available sight distances, variation of design parameters, and reliability analysis in transportation. CHAPTER 3 displays the concept of the reliability theory and the framework that was suggested for the geometric design using such theory. The chapter also describes the computer program that was developed in this research to evaluate the sight distance along with the anticipated hazard. CHAPTER 4 presents a new methodology to calculate SSD avoiding several approximations in current design practice. In addition, both analytical and simulation reliability techniques were applied to express the SSD distribution at a certain road section. As a part of the new methodology, field measurements were also collected to evaluate the friction coefficient between the pavement surface and vehicle tyres at different surface conditions.

CHAPTER 5 displays the two and three dimensional approaches to calculate the available sight distance. The chapter also presents the analytical and simulation reliability techniques to describe the distribution of such distance. In CHAPTER 6, the reliability theory was used to evaluate the hazard using the percentage of drivers who may suffer insufficient sight distance. In this regard, a case study was used to show the applicability and usefulness of the developed methodology. CHAPTER 7 presents a new reliabilitybased approach to locate lateral obstructions along three dimensional alignments. The new methodology replaces the current deterministic approach. Finally, the main conclusions and recommendations are highlighted in CHAPTER 8. 


\section{CHAPTER 2}

\section{LITERATURE REVIEW}

This chapter presents the previous research and current practice of highway geometric design that is relevant to the topic of this thesis. Section 2.1 covers different types of required sight distances (RqSDs) such as stopping sight distance, decision sight distance, and passing sight distance. In Section 2.2, methods of determining available sight distances (AvSDs) either from the horizontal alignment, vertical alignment, or in the 3D space will be presented. Section 2.3 shows possible consequences when AvSD on a road segment is limited compared to RqSD. Section 2.4 contains recommendations of frequency distributions of different design parameters. Finally, Section 2.5 represents general risk assessment analysis and reliability analysis in transportation engineering.

\subsection{Required Sight Distance (RqSD)}

The RqSD is the distance that drivers may need to stop, pass a slower vehicle, or take a right decision. Among those types, the stopping sight distance (SSD) is the minimum requirement that must be provided on all road categories while the passing sight distance (PSD) is required only on two-lane roads. In addition, the decision sight distance (DSD) is usually provided to give drivers enough time to be able to adapt their speeds and operate safely at a specific situation. The following few sections describe past practice to calculate each of the three types. 


\subsubsection{Stopping Sight Distance (SSD)}

The SSD is defined as the distance that is "long enough to enable a vehicle travelling at or near the design speed to stop before reaching a stationary object in its path" (Fambro 1997). In AASHTO (1994) and earlier editions, the SSD model shown in Equation 2-1 was formulated based on basic laws of physics.

$$
S S D=0.278 V t+\frac{V^{2}}{254(f \pm G)}
$$

where; $S S D=$ stopping sight distance (m); $t=$ perception and reaction time (second); $V=$ initial speed $(\mathrm{km} / \mathrm{h}) ; f=$ coefficient of friction between tires and roadway; and $G=$ percent grade divided by 100 .

The equation assumes that a driver would travel at a constant speed $(V)$ for time equal to the perception and reaction (PR) time $(t)$. Then, a braking distance is travelled while a vehicle's brake is actually applied until a complete stop occurs. The PR time is the time needed by a driver to detect an object and initiate the execution of a decided action. A value of 2.5 seconds for $t$ was stated in AASHTO (1994) to be adequate and larger than the average time that could be needed by nearly all drivers under most highway conditions. As for the friction coefficient, a wide variation was assumed due to several factors such as "air pressure of tires, composition of tires, type and condition of pavement surface, and the presence of moisture, mud, snow, or ice" (AASHTO 1994). Friction coefficients were, however, suggested for different values of design speeds based on the worst driving conditions such as wet pavements, pavements that approach the end of their useful lives, and worn tires. The suggested values were stated to be conservative and appropriate for the calculation of SSDs. 
It should be mentioned that the design values that were presented in AASHTO (1994) reflect passenger cars. Therefore, the suitability to apply these values to trucks is questionable since trucks' SSDs are normally longer than those of passenger cars. However, it was stated that the ability of a truck operator to see the vertical features of the obstruction farther than the passenger car's driver would balance the need for a longer SSD. Even though, this point was mentioned to have a little effect in few situations such as the end of a long downgrade where trucks' speeds closely approach or exceed passenger cars' speeds. In such situations, AvSD is encouraged to exceed the recommended SSD.

The AASHTO (1994)'s equation of SSD was evaluated by Harwood et al. (1989) to assess its applicability to large trucks. It was mentioned that considering the characteristics of passenger cars rather than trucks might neglect the need for a longer braking distance and hence increase the potential of collisions. The authors assessed the capabilities of two braking systems including the conventional brake and the antilock brake systems. Three scenarios were also suggested including (1) tractor-trailer truck with a conventional brake system and the worst-performing driver, (2) tractor-trailer truck with a conventional brake system and the best-performing driver, and (3) tractortrailer truck with an antilock brake system. Data from a previous study by Fancher (1986) were reused with a minor change in the pavement surface properties. It was found that trucks equipped with antilock brake systems are the only type that has values close to those recommended by AASHTO (1984) for passenger cars while, for the other two types, braking distances were found to be significantly longer. 
The AASHTO (1994)'s model was also criticized by Fambro (1997) for the reason that its parameters are not representative of the driving environment and safe driving behaviour. It was also mentioned that there is a difficulty to justify and validate the model. A revised model was then suggested as shown in Equation 2-2 considering the driver's deceleration rate $(a)$ instead of the friction coefficient $(f)$. It is worthy to mention that AASHTO (2004) replaced the old model with the new one based on Fambro (1997)'s study.

$$
S S D=0.278 V t+\frac{V^{2}}{254\left(\frac{a}{9.81} \pm G\right)}
$$

where; $a=$ deceleration rate $\left(\mathrm{m} / \mathrm{s}^{2}\right)$.

Similar to the old model, the SSD is a summation of two distances. The first distance is common between the two models and was said to vary widely with the normal variation of several factors such as "the distance to the object, the visual acuity of the driver, the natural rabidity with which the driver reacts, the atmospheric visibility, the type and the condition of the roadway, and nature of the obstacle." On the other hand, a deceleration rate of $3.4 \mathrm{~m} / \mathrm{s}^{2}$ was assumed to meet the friction on most wet pavement surfaces and the capabilities of most vehicle braking systems. Although near-worst values of the parameters in this equation were thought to provide most drivers with adequate SSD, there is still a need to quantify the risk when vehicles require larger values than such distance.

Fambro et al. (2000b) evaluated the new SSD model against the old model that was presented in AASHTO (1994) and other models in the literature. It was stated that 
the recommended SSD values rely on a below-average driver, vehicle, and roadway characteristics. However, the probability of having simultaneous critical values for all these parameters could be extremely small. This would create a considerable margin of safety when the recommended SSD values in AASHTO (1994) are considered in the geometric design. Comparisons between different guidelines in different countries were also conducted and revealed that AASHTO's SSD and subsequently lengths of vertical curves are relatively longer than those used in most other countries. Generally, it was recommended to provide longer SSDs than those resulting from the proposed equation when horizontal sight restrictions occur on long downgrades.

\subsubsection{Decision Sight Distance (DSD)}

The DSD is required when complex decisions have to be carried out, especially when information is difficult to attain. In such cases, drivers need longer SD to adapt their operating speeds and perform appropriate actions. Therefore, SSD may not be satisfactory and might subsequently affect operational and safety performance. TAC (1999) and AASHTO (2004) divided DSD into five categories; (1) avoidance manoeuvre $A$ : stop on rural roadway, (2) avoidance manoeuvre $B$ : stop on urban roadway, (3) avoidance manoeuvre $C$ : speed/path/direction change on rural roadway, (4) avoidance manoeuvre $D:$ speed/path/direction change on suburban roadway, and (5) avoidance manoeuvre $E$ : speed/path/direction change on urban roadway. It was stated in TAC (1999) that situations where DSD might be required are complex interchanges and intersections, locations where unusual manoeuvres occur, or locations where significant 
changes to the roadway cross-section take place. In such situations, control actions are required rather than stopping.

As stated by Hassan and Easa (2000), a special case of DSD is the preview sight distance (PVSD) that was firstly proposed by Gattis and Duncan (1995). Hassan and Easa (2000) stressed the importance of the highway coordination in order for a driver to see, perceive, and react to a horizontal curve before its beginning. If inadequate distance was given to drivers, deterioration in safety performance could occur. The authors presented a case study where seven collisions on Highway 11 , Ontario, were linked to insufficient SD between May 1989 and January 1993. The study also introduced a framework to estimate required PVSD based on the laws of kinematics and based on physical modelling and computer animation. Figure 2-1 shows the two PVSD components on both tangent and curve; referred to as $S_{1}$ and $S_{2}$.

In addition, Equation 2-3 through Equation 2-5 summarize the proposed methodology to calculate PVSD. The curve radii used in this study ranged from $500 \mathrm{~m}$ to $2000 \mathrm{~m}$ and other values were recommended to be cautiously considered. It was also stated that $2 \mathrm{D}$ models to predict operating speeds may be inadequate and should be replaced by 3D models. Finally, further studies were recommended on the drivers' behaviours while approaching horizontal curves with limited PVSD. 


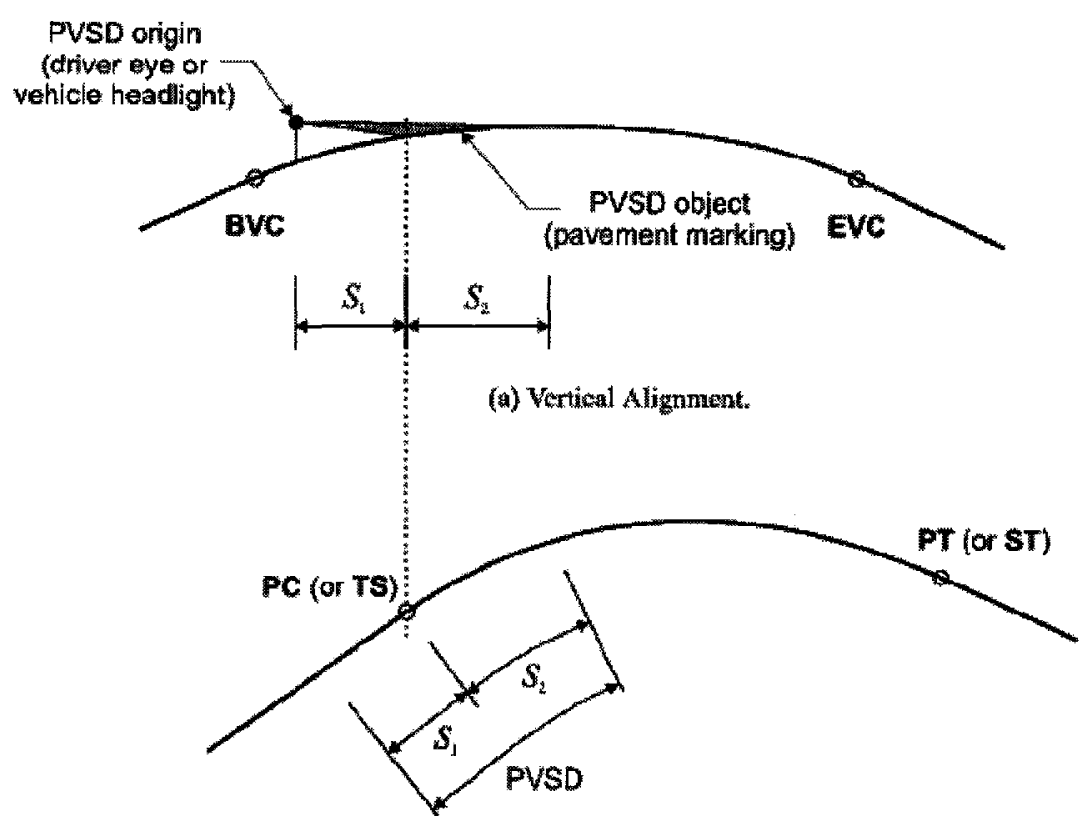

(b) Horizotial Alignment.

Figure 2-1: Components of Required PVSD (Hassan and Easa 2000).

PVSD on Tangent
$S_{1}=\left\{\begin{array}{lr}L_{1}+L_{2} & \text { simple curve } \\ L_{1}+L_{2}-l_{s} & \text { spiral curve, } L_{2}>l_{s} \\ L_{1} & \text { spiral curve, } L_{2} \leq l_{s}\end{array}\right.$
$L_{1}=0.278^{*} P^{*} V_{T}$


where; $P=$ perception and reaction time $(\mathrm{sec}) ; V_{\mathrm{T}}=$ tangent operating speed $(\mathrm{km} / \mathrm{h}) ; V_{\mathrm{C}}=$ curve operating speed $(\mathrm{km} / \mathrm{h}) ; d=$ deceleration rate $\left(\mathrm{m} / \mathrm{s}^{2}\right) ; l_{\mathrm{s}}=$ spiral length $(\mathrm{m}) ; \delta$ $=$ deflection angle; $A=$ spiral parameter $(\mathrm{m}) ; G=0$ for simple curves and 1 for spiral curves.

\subsubsection{Passing Sight Distance (PSD)}

Providing enough PSD is required only on two-lane roads, where drivers might legally occupy the opposing lane in order to pass a slower vehicle. Drivers should have the ability to see far enough to decide the possibilities of executing such a manoeuvre. In TAC (1999) and AASHTO (2004), PSD is divided into four elements as shown in Figure

2-2. These elements were defined as follows:

1. $d_{1}$ : initial manoeuvre distance; that is the distance travelled during the PR time plus the distance travelled while the passing vehicle traverses from the trailing position to the opposing lane.

2. $d_{2}$ : the distance travelled by passing vehicles along the opposing lane.

3. $d_{3}$ : clearance length; that is the suggested safe distance between the passing vehicle and the opposing vehicle at the end of the manoeuvre. This distance was reported to vary from $30 \mathrm{~m}$ to $90 \mathrm{~m}$.

4. $d_{4}$ : the distance travelled by the opposing vehicle starting at the time it is seen by the passing vehicle. This value is recommended to be two thirds of $d_{2}$ as the opposing vehicle is assumed to travel at the same speed as the passing vehicle. 


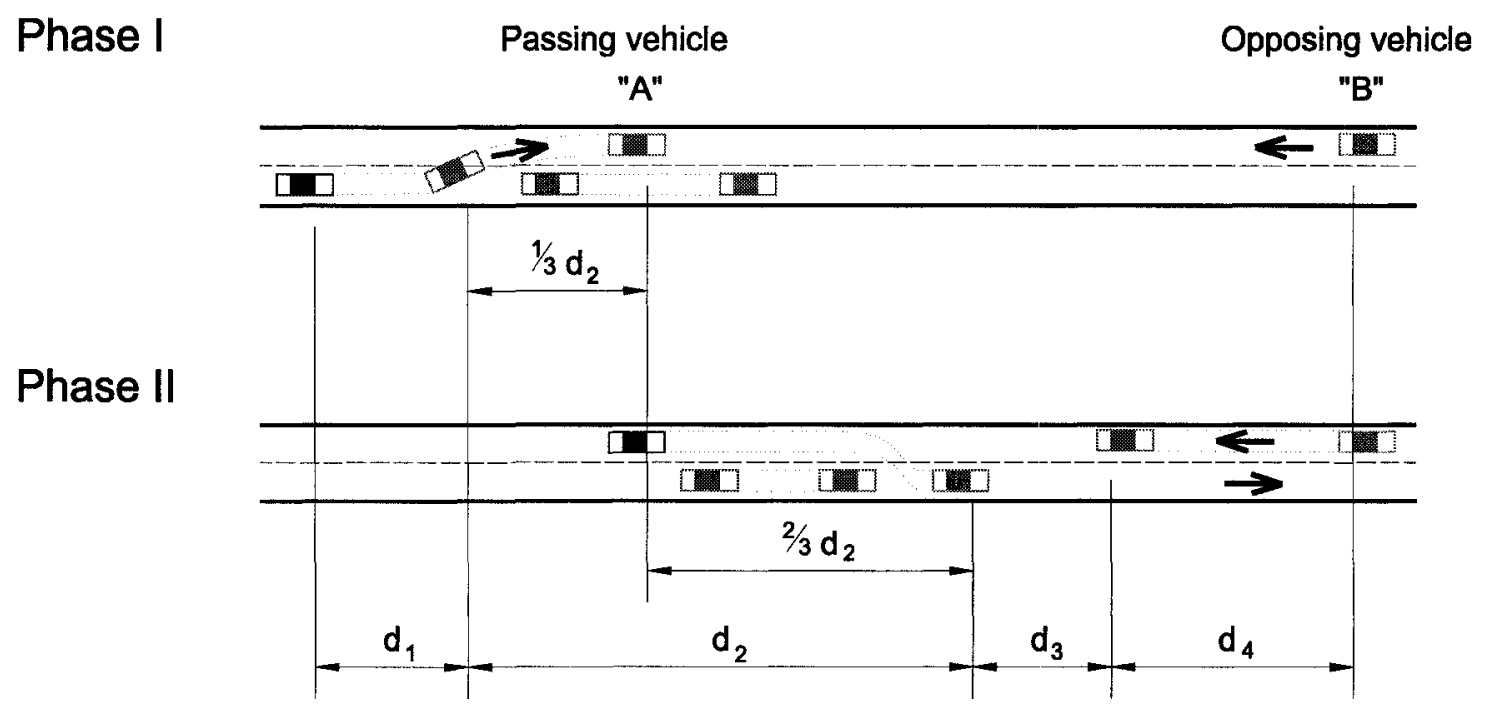

Figure 2-2: Elements of PSD (Adapted from TAC 1999).

As stated in TAC (1999), the required PSD (i.e., the summation of these four distances) is the minimum for a passenger car to pass another passenger car. This distance increases when a passenger car is passing a truck, a truck is passing a passenger car, and it reaches its maximum when a truck is passing another truck. It was mentioned that there is a lack of understanding of the relationship between the safety and passing manoeuvres. However, collisions involving passing manoeuvres were reported in TAC (1999) to contribute only $2 \%$ of all collisions on two-lane rural roads. It was also recommended to provide passing lanes whenever passing manoeuvres are practically difficult.

Harwood and Glennon (1989) evaluated PSD design and passing zone length criteria for both passenger cars and trucks. It was stressed that the AASHTO (1984) design guide used field data dated back to 1938 and 1941, which were again validated in 
1958, to calculate the required PSD. In order to account for different vehicle types, four passing scenarios were modeled; including (1) passenger car passing passenger car, (2) passenger car passing truck, (3) truck passing passenger car, and (4) truck passing truck. As shown in Figure 2-3, the four scenarios gave lower values of PSD than those recommended by AASHTO (1984). However, only the values associated with the first scenario agreed with corresponding values stated in the Manual on Uniform Traffic Control Devices for Streets and Highways (MUTCD 1978) while other scenarios were found to be greater.

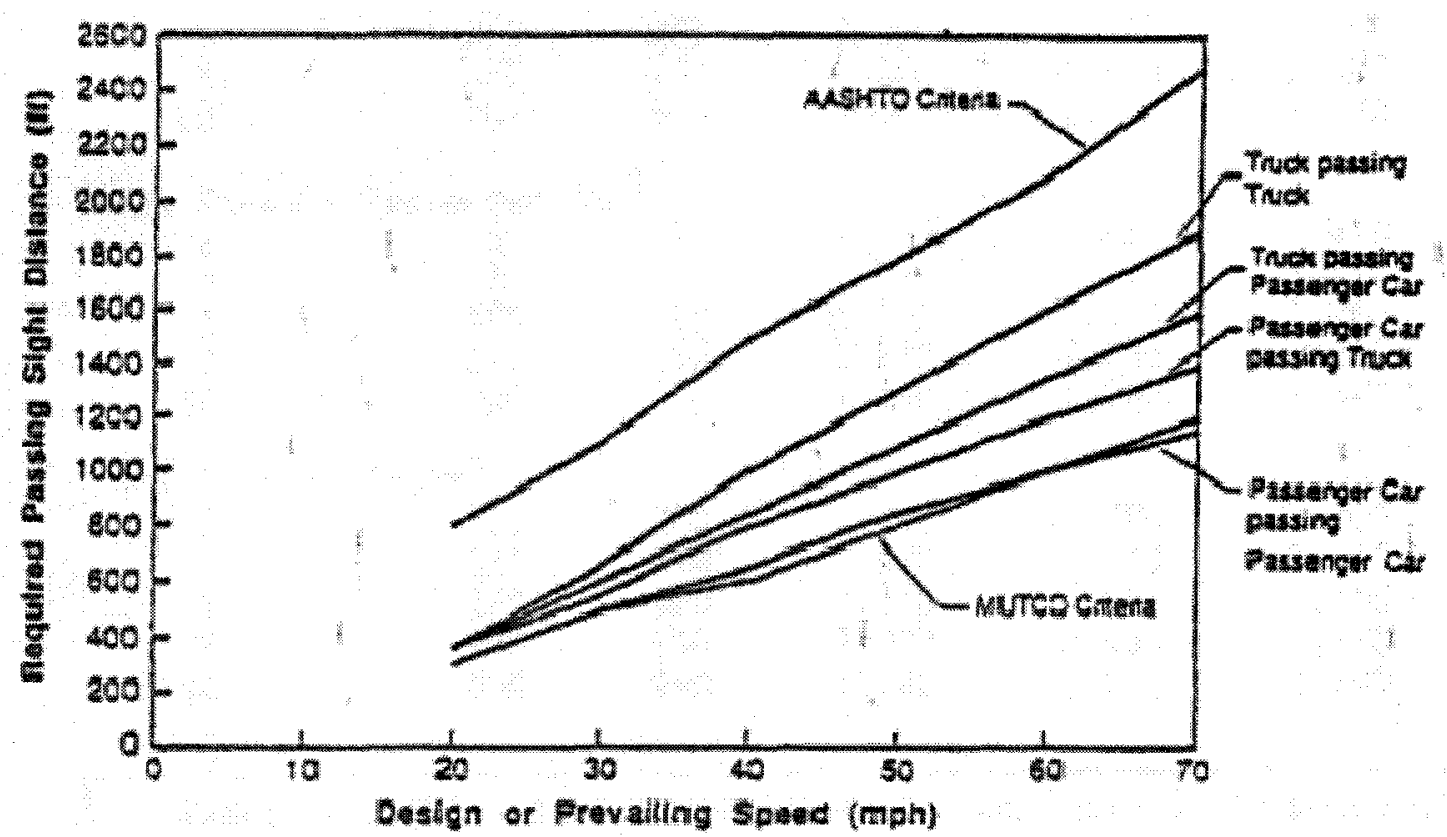

Figure 2-3: Required PSD for Passenger Car and Trucks in Comparison with Design Guides (Harwood and Glennon 1989).

Wang and Cartmell (1998) divided the passing manoeuvre into three elements rather than the four elements listed in TAC (1999). As presented in Figure 2-4, the first element represents the distance travelled by the passing vehicle from the beginning of the 
overtaking manoeuvre to the point where the passing vehicle's head is aligned with the tail of the impeding vehicle. As stated, the path of this element is fitted with a quintic polynomial. The second element represents the distance travelled by the passing vehicle while occupying the opposing lane until the passing vehicle's tail is aligned with the head of the impeding vehicle. This portion was described as a straight line. The third and last element represents the distance from the end of the second element to the end of the overtaking manoeuvre, which was again described by a quintic polynomial. Equations that formulate these three distances were presented and values of PSD were finally compared with those of AASHTO (1994). It was concluded that AASHTO (1994)'s values are generally conservative for modern vehicles. It was also stated that a PSD would increase considerably with the increase of the vehicle length.

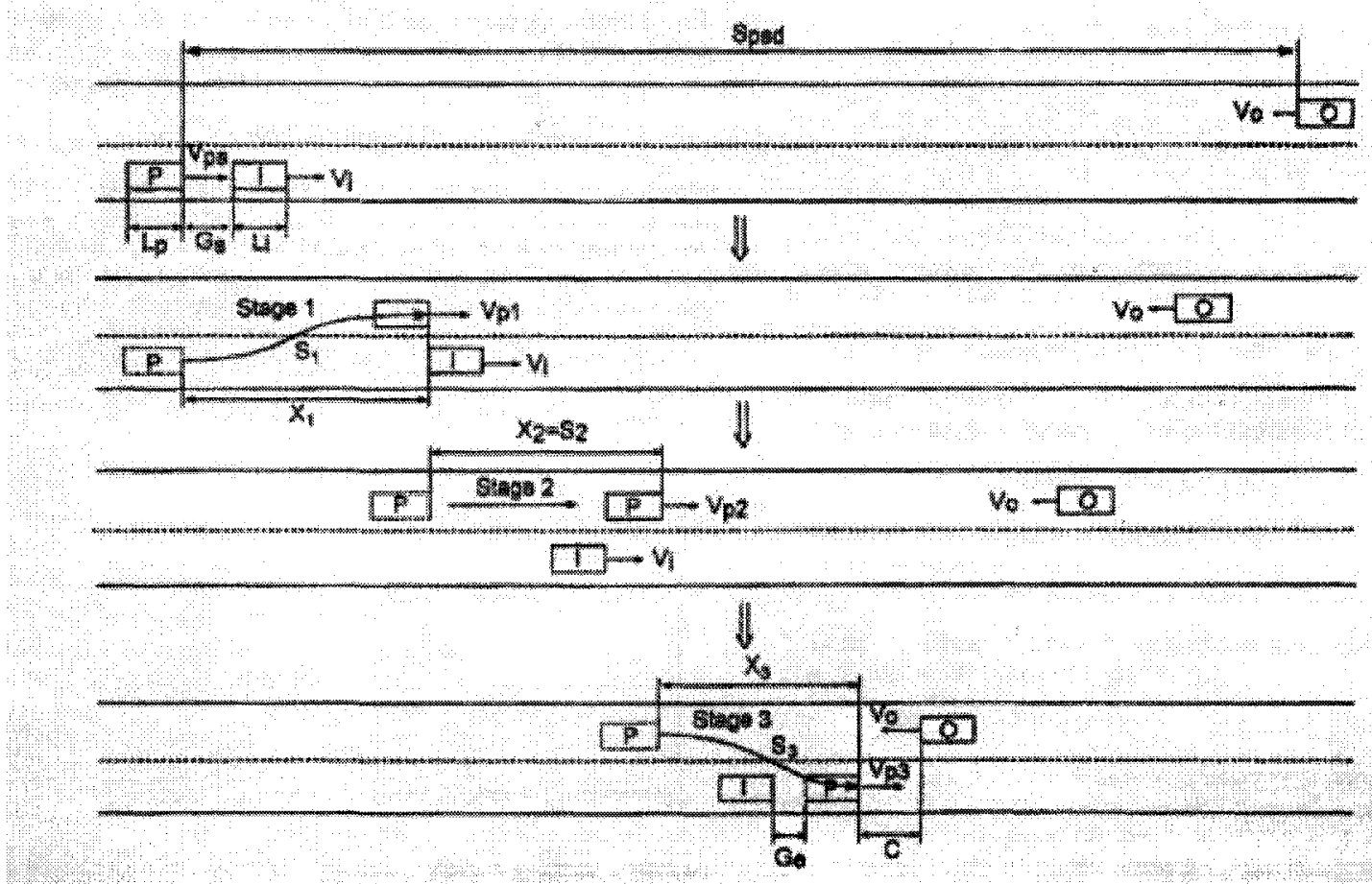

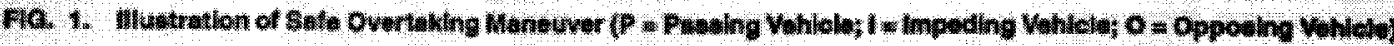

Figure 2-4: Elements of PSD (Wang and Cartmell 1998). 
The study done by Jenkins and Rilett (2006) is one of the recent studies that assessed the effect of several factors on PSD. The study's objectives included (1) classification of factors that have a great impact on PSD and (2) formulating a PSD model that can be used in traffic simulation programs. Although the proposed model assumes that a PSD is divided into four separate distances similar to the concept followed in TAC (1999) and AASHTO (2004), the definitions of these elements were slightly different. Equation 2-6 shows briefly the total PSD as presented in this study.

$$
P S D=\bar{s}_{p} t_{1}+G_{s}+L_{i}+\bar{s}_{i} t_{2}+L_{p}+G_{E}+C+\bar{s}_{o}\left(t_{1}-t_{2}\right) \quad \text { Equation 2-6 }
$$

where; $t_{1}=$ time taken by the passing driver to decide to pass and move the vehicle towards the left; $t_{2}=$ time the passing vehicle occupies the left lane; $L_{\mathrm{v}}=$ length of vehicle $\mathrm{v} ; s_{\mathrm{v}}=$ speed of vehicle $\mathrm{v} ; \overline{s_{p}}=$ average speed of vehicle $\mathrm{v} ; \overline{a_{v}}=$ average acceleration rate of vehicle $\mathrm{v} ; G \mathrm{~s}=$ gap between the passing and impeding vehicles at the start of $t_{2} ; G_{\mathrm{E}}=$ gap between the passing and impeding vehicles at the end of $t_{2} ; C=$ clearance between the passing and oncoming vehicles at the end of $t_{2} ; G_{\mathrm{S}}+L_{\mathrm{i}}+L_{\mathrm{p}}+G_{\mathrm{E}}=$ space needed to be gained by the passing vehicle.

\subsection{Available Sight Distance (AvSD)}

The AvSD at a specific road section could be horizontally restricted by physical features along the road side such as buildings, trees, side slopes, and barriers. The AvSD could also be restricted vertically by the road surface of a crest vertical curve or an overpass crossing a sag vertical curve. In all these cases, the determination of AvSD is essential since it would affect the geometric design of highway features. The following three sub-sections show the methodologies that are listed in current design guides and 
other relevant research to obtain AvSD from the horizontal alignment, vertical alignment, and combined alignment, respectively.

\subsubsection{Sight Distance Based on Horizontal Alignment}

In AASHTO (2004), values of AvSD are determined from either horizontal or vertical alignment projections separately. As shown in Figure 2-5, an obstacle that horizontally limits the sight line is located at certain horizontal sight offset (HSO) from the centreline of the inner lane. Based on the geometry of the road section shown in this figure, the relationship between the components of AvSD was formulated as presented in Equation 2-7.

$$
H S O=R\left[1-\cos \frac{28.65^{*} S}{R}\right]
$$

where; $S=$ available sight distance; $R=$ curve radius; $H S O=$ horizontal sight offset.

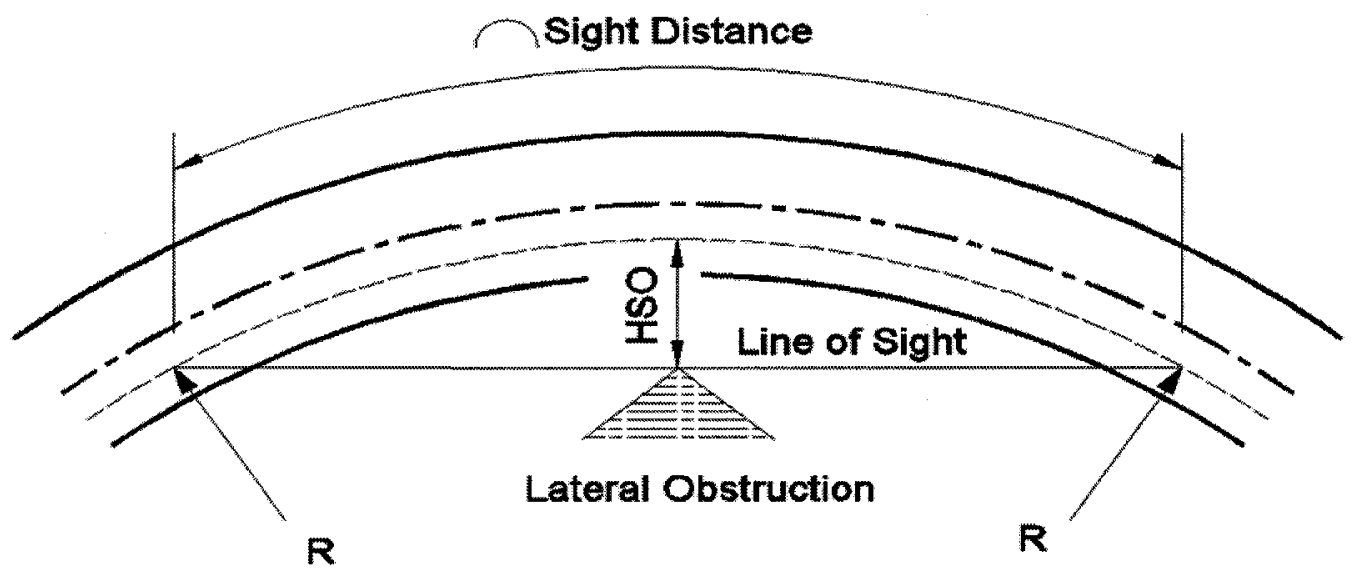

Figure 2-5: Determination of the Horizontal Sight Offset (Adapted from AASHTO 2004). 
It was mentioned that this method is only precise when both the vehicle and the object are located on the same simple horizontal curve. If one of them or both were beyond the curve limits, the obtained value of $H S O$ would be slightly larger than the one needed to satisfy a specific RqSD. However, it was stated that this additional clearance would not be significant in many instances. In addition, the term $S$ in this equation may be used as SSD or PSD, depending on the design target. For SSD criterion, it was emphasized that the designer should consider higher SSD values to allow for some friction to be used laterally on horizontal curves.

In addition, Hassan et al. (1995) proposed analytical procedures to check AvSD considering the case of a continuous lateral obstruction. The method was said to be valid for any combination of straight segments, circular curves, and spiral curves. The procedures were programmed using Microsoft Quick Basic to locate no-passing zones on two-lane highways. Moreover, the study by Easa and Hassan (1997) examined AvSD during nighttime based on vehicle headlights, referred to as headlight sight distance (HLSD). As stated, HLSD is limited by the angle of the horizontal light spread ( $\alpha$ ) as well as existing lateral obstructions. This distance was calculated using proposed steps for three different cases; (1) continuous obstruction on a circular curve, (2) continuous obstruction on a spiral curve, and (3) single obstruction. Two graphs were provided for the relationship between lateral clearance and curve radius at different values of speeds and at $\alpha$ equal to $10^{\circ}$ and $20^{\circ}$. The graphs were considered beneficial to check SSD against HLSD, as shown in Figure 2-6 for $\alpha$ equal to $20^{\circ}$. 


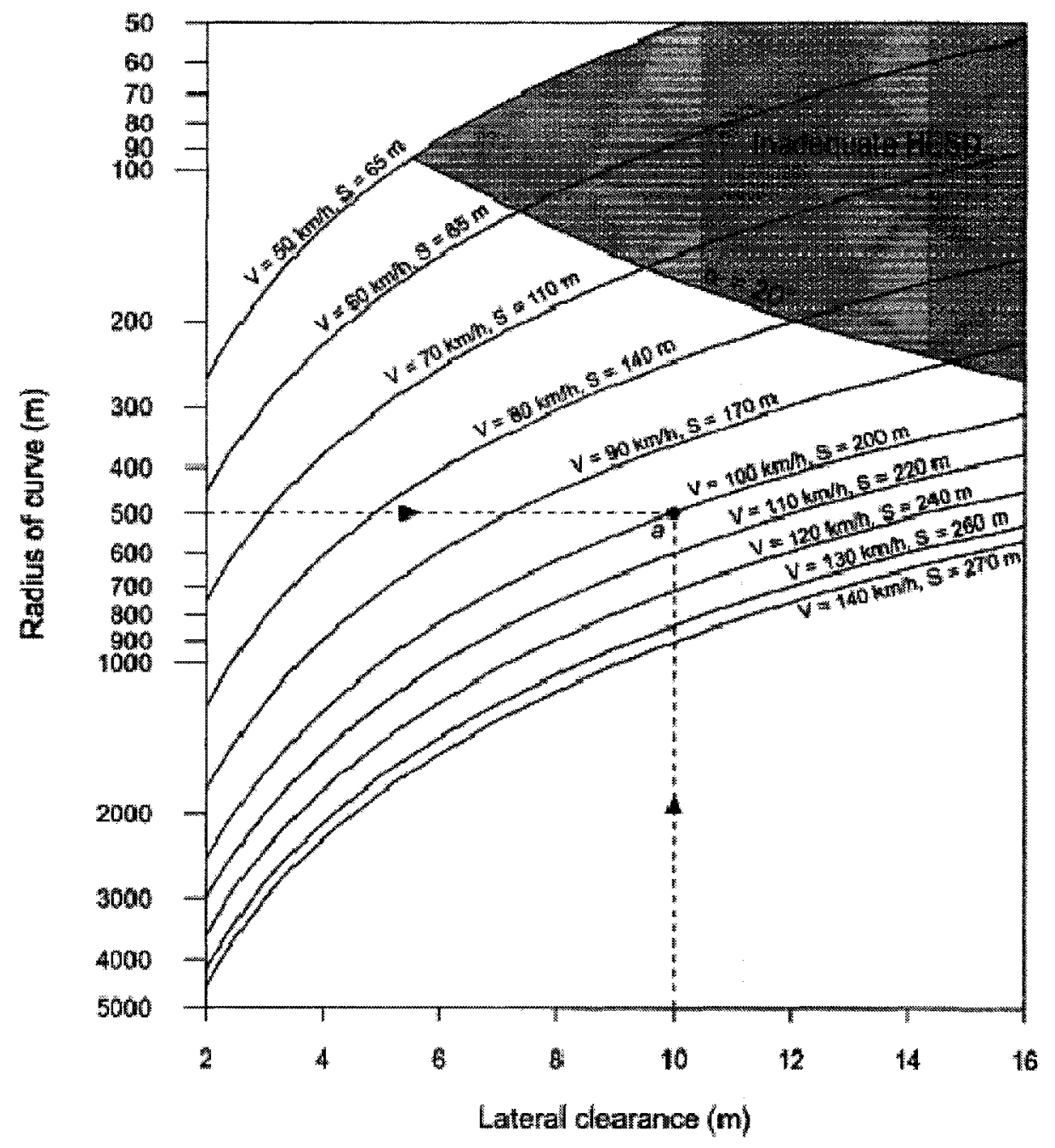

Figure 2-6: Check of HLSD on Simple Horizontal Curves; $\alpha=20^{\circ}$ (Easa and Hassan 1997).

Lovell (1999) also discussed a method to calculate AvSD along a highway alignment automatically. Only the horizontal alignment was used even though it was mentioned that AvSD should be calculated based on combined horizontal and vertical road geometry. The study aimed mainly at locating no-passing zones along highways. The alignment was assumed to consist of a smooth series of connected tangent sections, circular curves, and transition spiral curves. The method develops parametric curves to 
represent (1) the centerline of the roadway, (2) the center of the travelled lane, and (3) the lateral sight obstructions in the Cartesian space. The procedures were said to be valid for the case where only one lane exists in the direction of interest. In addition, the algorithm assumes that the highway is homogeneous in that direction and is located in a flat area. The results were stated to be inaccurate near the downstream end of an alignment. This problem was overcome by coding a longer section of the alignment than required.

\subsubsection{Sight Distance Based on Vertical Alignment}

As mentioned earlier, AvSD could be calculated using the criteria of either sag or crest vertical curves. As shown in Figure 2-7, crest curves could restrict AvSD when sharp curves are implemented. Therefore, the length of a crest curve has to be long enough to enable drivers to recognize other vehicles and obstacles on the other side of the curve. This would help perform proper driving actions especially at high operating speeds. As stated in AASHTO (2004), Equation 2-8 and Equation 2-9 describe the relationship between the curve criteria and AvSD for both cases; (1) AvSD is less than the curve length, and (2) AvSD is greater than the curve length, respectively.

$$
\begin{array}{lll}
L>S ; & L=\frac{A S^{2}}{100\left(\sqrt{2 h_{1}}+\sqrt{2 h_{2}}\right)^{2}} & \text { Equation 2-8 } \\
L<S ; & L=2 S-\frac{200\left(\sqrt{h_{1}}+\sqrt{h_{2}}\right)^{2}}{A} & \text { Equation 2-9 }
\end{array}
$$

where; $L=$ length of crest curve (m), $S=\operatorname{AvSD}(\mathrm{m}), A=$ algebraic difference in grades $(\%), h_{1}=$ height of eye above roadway surface $(\mathrm{m})$, and $h_{2}=$ height of object above roadway surface $(\mathrm{m})$. 

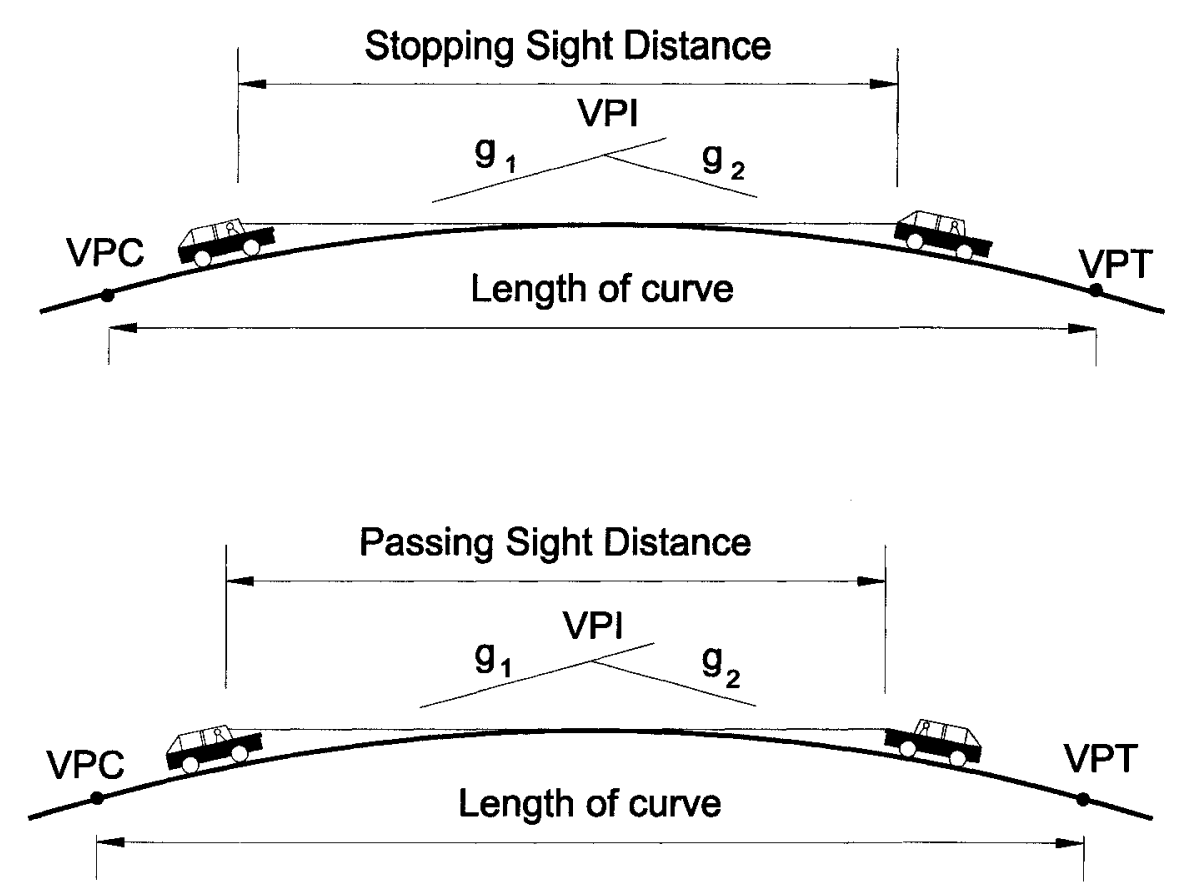

Figure 2-7: Sight Distance on Crest Vertical Curves (Adapted from AASHTO 2004).

As for sag vertical curves, AvSD is usually calculated to ensure safe operations during nighttime as the entire curve is normally seen by road users during day-time. The method relies on the divergence degree of a vehicle's light that enables drivers to see ahead of the vehicle. This visible distance increases with increasing the length of the sag curve. As shown in Equation 2-10 and Equation 2-11, two formulas were listed in AASHTO (2004) in order to determine minimum lengths of sag curves for two cases; (1) AvSD is less than the curve length, and (2) AvSD is greater than the curve length, respectively.

$$
\begin{array}{lll}
L>S ; & L=\frac{A S^{2}}{200\left(h_{3}+S \tan \alpha\right)} & \text { Equation 2-10 } \\
L<S ; & L=2 S-\frac{200\left(h_{3}+S \tan \alpha\right)}{A} & \text { Equation 2-11 }
\end{array}
$$


where; $L=$ length of sag curve, $S=\operatorname{AvSD}(\mathrm{m}), A=$ algebraic difference in grades (\%), $h_{3}=$ headlight height $(\mathrm{m}), \alpha=$ upward divergence angle of the light beam from the longitudinal axis of the vehicle (degree).

AASHTO (2004) also presented another criterion to determine the length of a sag vertical curve that is crossed by an overpass. In this method, a clearance $C$ between the pavement surface and the overpass structure is assumed. Such structure would vertically restrict the driver's line of sight as shown in Figure 2-8. Equation 2-12 was also provided to calculate the minimum length of such sag curve.

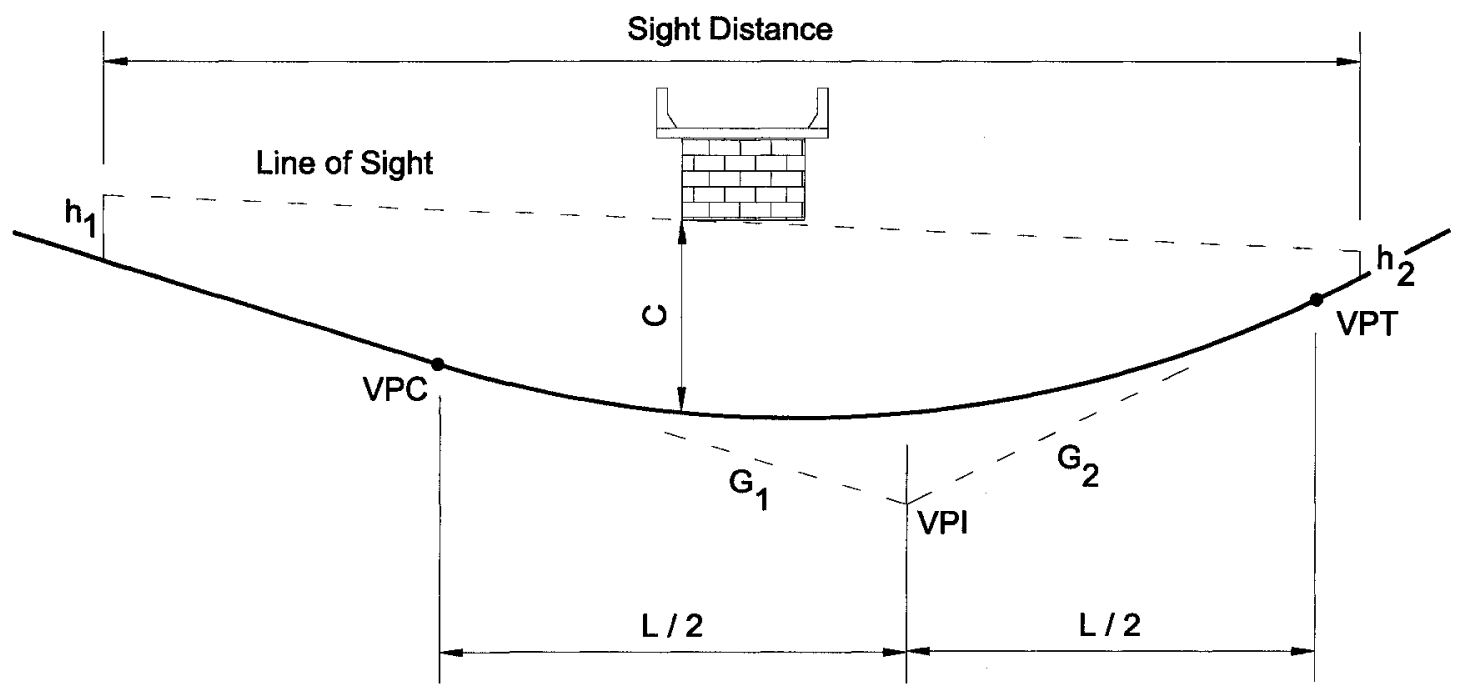

Figure 2-8: Sight Distance of Sag Vertical Curves (Adapted from AASHTO 2004).

$L=2 S-\frac{800\left[C-\left(\frac{h_{1}+h_{2}}{2}\right)\right]}{A}$

Equation 2-12

where; $C=$ vertical clearance $(\mathrm{m})$. 
Easa et al. (1996) studied AvSD at complex combinations of vertical curves. It was mentioned that previous research focused on the determination of AvSD on only simple and isolated sag and crest vertical curves with long tangents. The proposed method used a system of $X$ (highway centerline) and $Z$ (elevation) axes to draw the highway centreline. Equations were suggested to model the vertical alignment elements. The AvSD was then determined by assuming initial AvSD and checking the intersection points between the line of sight and the road surface. The assumed AvSD value is then decreased or increased till a single intersection point is finally obtained (the line of sight becomes tangent to the crest curve). This task was computerized using two computer programs based on programmable iterative models to determine the profile of AvSD (see Figure 2-9).

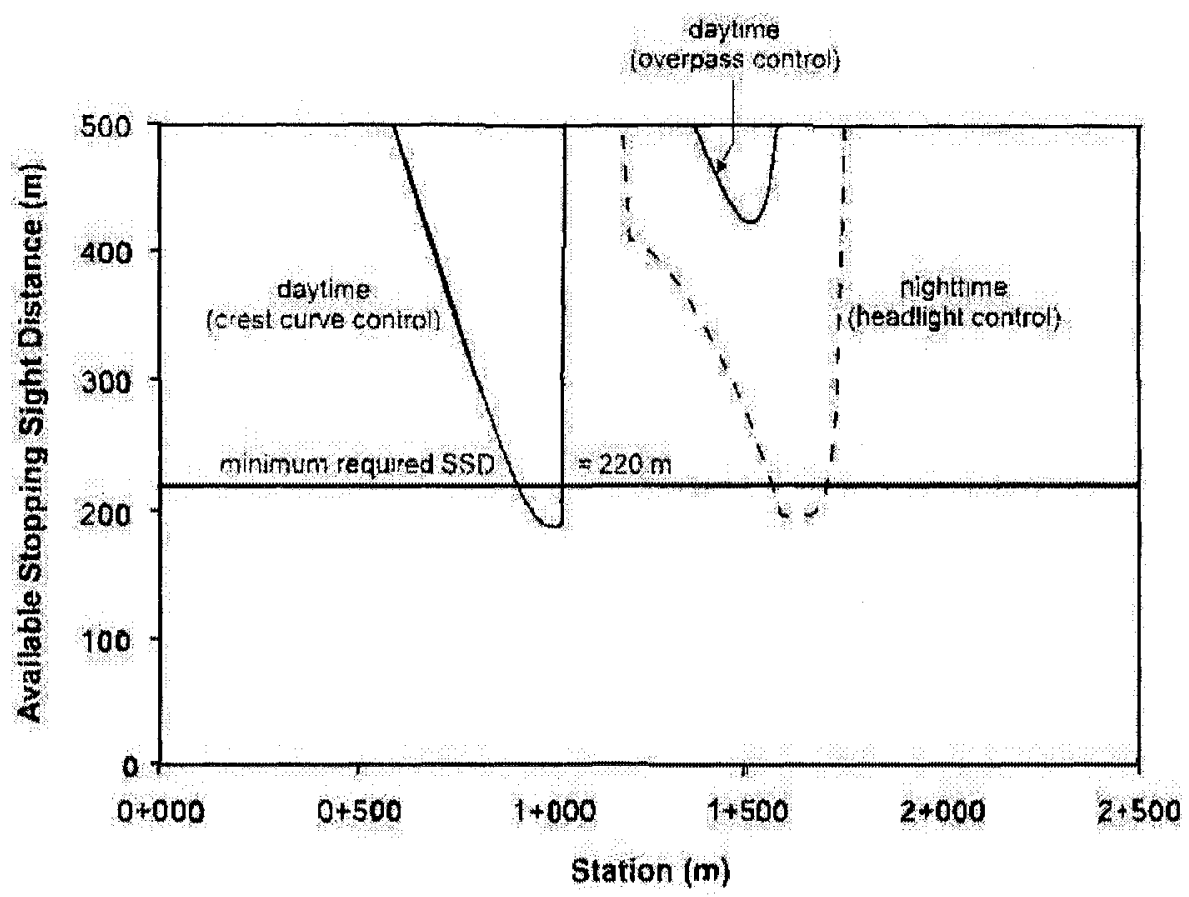

Figure 2-9: AvSD for Application Example (Easa et al. 1996). 
In addition, both Easa et al. (1996) and Easa and Hassan (1997) checked HLSD on vertical alignments where dips would exist due to different arrangements of crest and sag vertical curves. In these dips, the road surface may not be covered by the vehicle's light and would not subsequently appear to drivers during nighttime. These dips may also be hidden to drivers with lower risk levels during day-time. Modelling attempts and computer programs were also used to check such dips automatically.

\subsubsection{Sight Distance Based on Combined Alignment}

After the recent criticism of the current 2D approach to obtain AvSD, efforts were devoted to investigate new methodologies that consider the combination of horizontal and vertical road alignments. In this regard, Hassan et al. (1996) developed a new model based on the curved parametric elements used in the finite element method. The methodology depends on fitting the road surface and sight obstructions with 3-node, 4node, 6-node, and 8-node elements. The sight line then connects a point that represents the driver's eye to an object located at distance $S$ ahead of this point. If the sight line intersects with any of the road elements, AvSD is then assumed to be overestimated and therefore a lower value is tried. In case the sight line does not intersect with any of the road elements, the value of $S$ is then increased until one point of intersection occurs (e.g., the sight line is tangent to any of the road elements). In such a case, $S$ is considered equal to AvSD at this highway section. It was stated that "ignoring the $3 D$ nature of the highway alignment may have serious consequences for the economy and safety of highways" (Hassan et al. 1996). 
Hassan and Easa (1998a) proceeded to calculate the headlight sight distance (HLSD) for 3D complex alignments. It should be noted that HLSD is the distance that is covered by a vehicle's headlight and must be, at least, equal to the SSD for the design purpose of sag vertical curves. The method proposed by Hassan and Easa (1998a) used the finite element technique to idealize the highway alignment into small elements for easy mathematical modelling. The study suggested that, at nighttime, drivers attempt to maintain their path relative to the road marking. Using the proposed method, HLSD can be calculated as the distance between the vehicle and the farthest point on the road marking that is covered by the light beam. It was stated that design of sag vertical curves using the 2D approach may underestimate its length to satisfy a specific RqSD.

Moreover, the study by Nehate and Rys (2006) is one of the recent studies that evaluated AvSD based on the combined alignment. The authors used the global positioning system (GPS) to collect road data in the form of latitude, longitude, and altitude at a time interval of one second. Data were then transformed to Cartesian coordinates that were further checked for outliers. A highway surface and sight obstructions were then described using parametric equations in the form of cubic $B$ splines. Using the horizontal and vertical projections of the sight line, AvSD was determined. The process was modelled using the Matlab programming language. The program was applied on a case study and both AvSD and RqSD were plotted in a graph where the deficient AvSDs can be visually allocated (see Figure 2-10). 


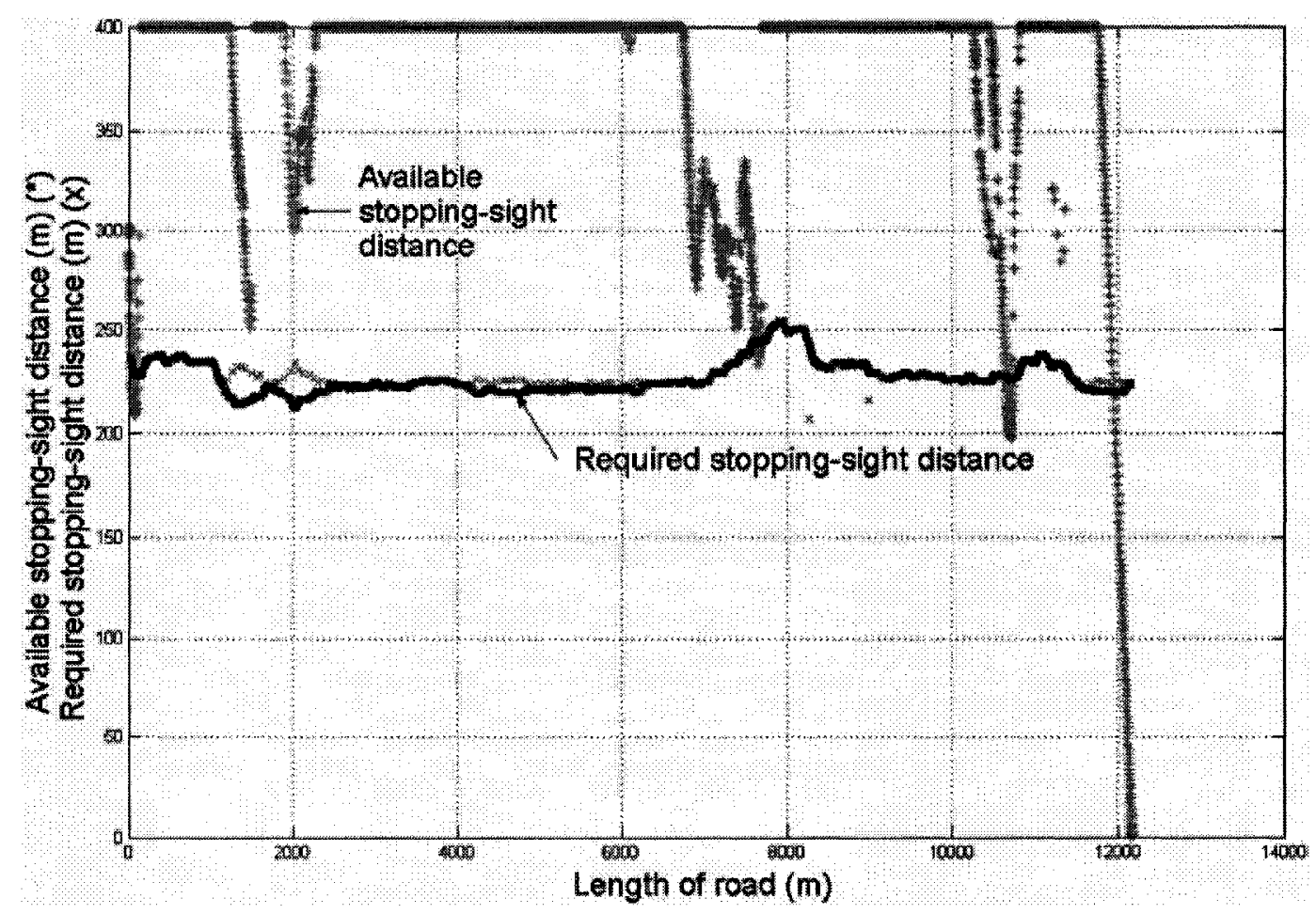

Figure 2-10: SSD versus Length of Road for K-177 in Riley Country, Kensus, U.S. (Nehate and Rys 2006).

\subsection{Impact of Limited Sight Distance}

The problem of limited AvSD might appear widely on old highways that have been upgraded to accommodate higher speeds. On such roads, a restricted AvSD would create potential hazards and significant safety impacts to road users. Several research studies presented probable consequences that would result from limited AvSD. Freedman et al. (1985) mentioned that an intersection which is just beyond a crest vertical curve is one of the most hazardous locations. In such locations, drivers would have insufficient time to execute proper actions while travelling at high speeds. It was also mentioned that 
a large portion of daily traffic is usually unfamiliar with the danger of such situations and subsequently do not exercise enough caution.

Urbanik et al. (1989) tested two large sets of data consisting of 222 road segments that included nearly 1500 collisions. The purpose of this study was to assess the safety effects of limited AvSDs on crest vertical curves. These collisions were filtered to isolate the impact of other geometric and operational conditions. The study segments were mentioned to have SSDs that are less than the AASHTO's minimum requirements for a 55-mph posted speed on all segments. It was found that sharp horizontal curves and intersections that are located within the limited-AvSD sections of crest vertical curves had significantly increased collision rates.

From the operational-impact standpoint, Fambro et al. (2000a) studied the effect of crest vertical curves with limited AvSD on operating speeds. The study focused on relatively old roadways in rolling terrain located in Washington, Texas, and Illinois states, as they had large number of limited-AvSD curves. Sites were selected in this study so that crest vertical curves would have AvSD less than the minimum SSD recommended by AASHTO for a design speed of $90 \mathrm{~km} / \mathrm{h}$ (i.e., $\mathrm{SSD}=130 \mathrm{~m}$ ). The study included both multilane and two-lane roadways with and without shoulders. In addition, three levels of traffic volumes were identified to capture the effect of the increase of volume on speed.

The speeds of individual vehicles were collected at a control area, where such speeds are freely selected by drivers. Speeds were also collected on crest curves as they were influenced by limited AvSD. The relationship between design and operating speeds was then analyzed assuming that a low design speed would indicate less AvSD. It was 
found finally that AvSD would significantly reduce speeds on crest vertical curves as shown in Table 2-1. Furthermore, several relationships were also suggested to predict $85^{\text {th }}$ percentile speed in terms of the design speed.

Table 2-1: Mean Speed Reductions Observed in Different Locations in Washington (WA), Taxes (TX), and Illinois (IL) (Adapted from Fambro et al. 2000a).

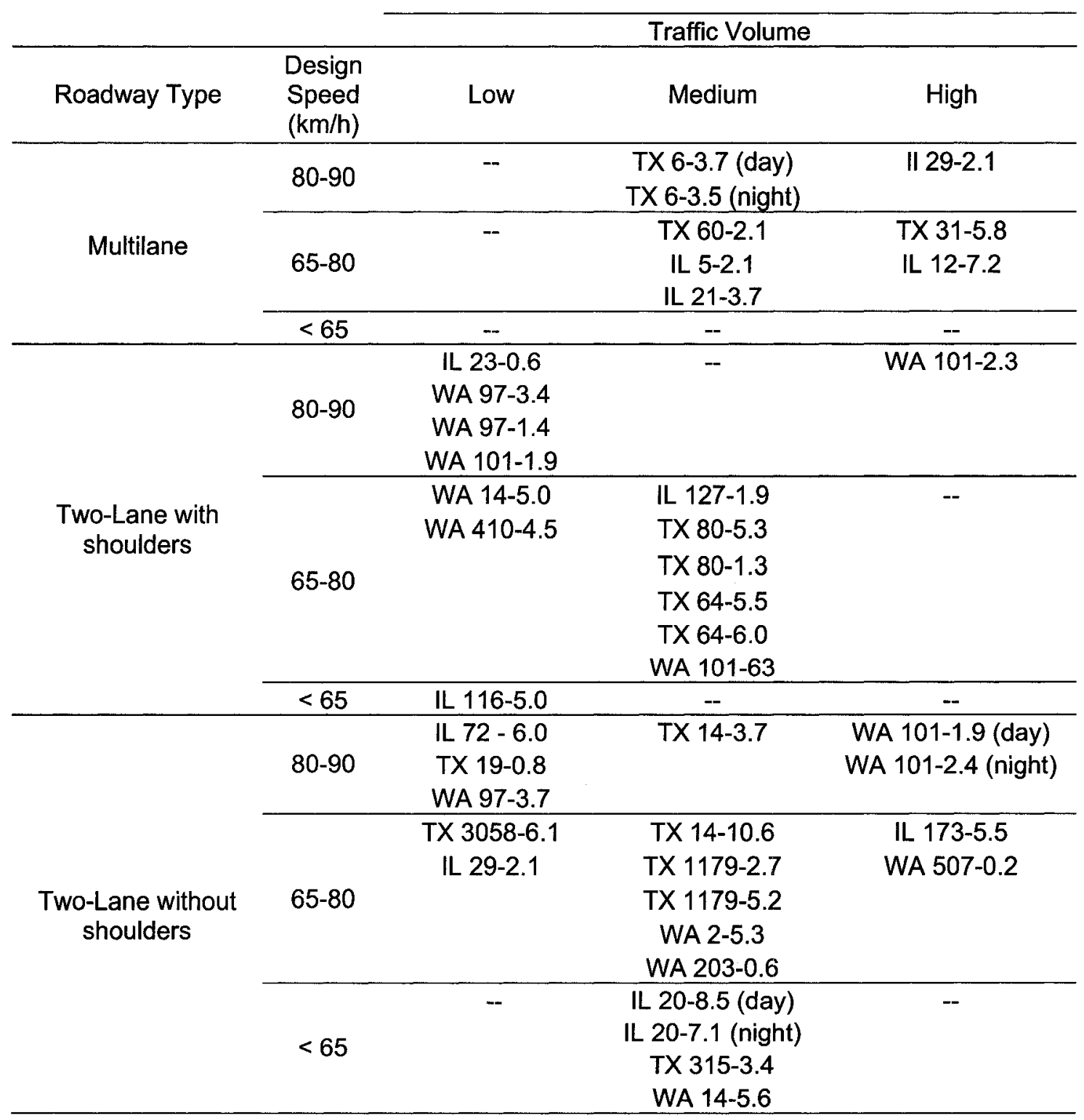


One of the very recent studies conducted on AvSD and its influence on drivers' behaviour is the one done by Discetti and Dell'Acqua (2008). The study evaluated the operating speed at road sections where the side obstructions dramatically restricts the AvSD (see Figure 2-11). Free-flow speeds were collected on a total of 44 curves located in Salerno province, Italy. One of the main findings of this study is the chart presented in Figure 2-12 that relates AvSD to the $85^{\text {th }}$ percentile speed (V85). The chart shows the relationship at four different radii $(60,90,180$, and $250 \mathrm{~m})$ and indicates that V85 may increase linearly with the increase in AvSD.

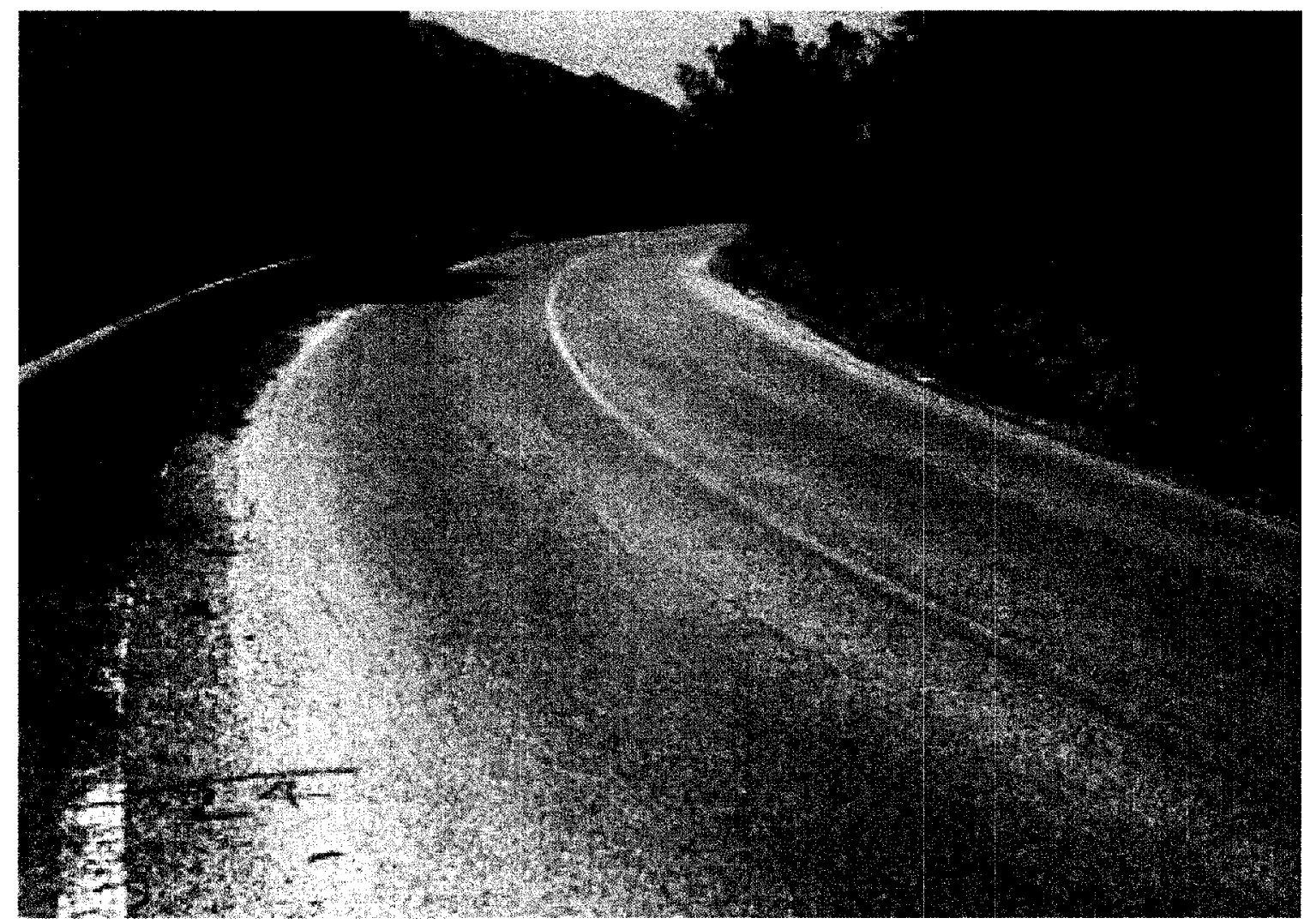

Figure 2-11: Limited Sight Distance on Road SS 166 (Discetti and Dell'Acqua 2008). 


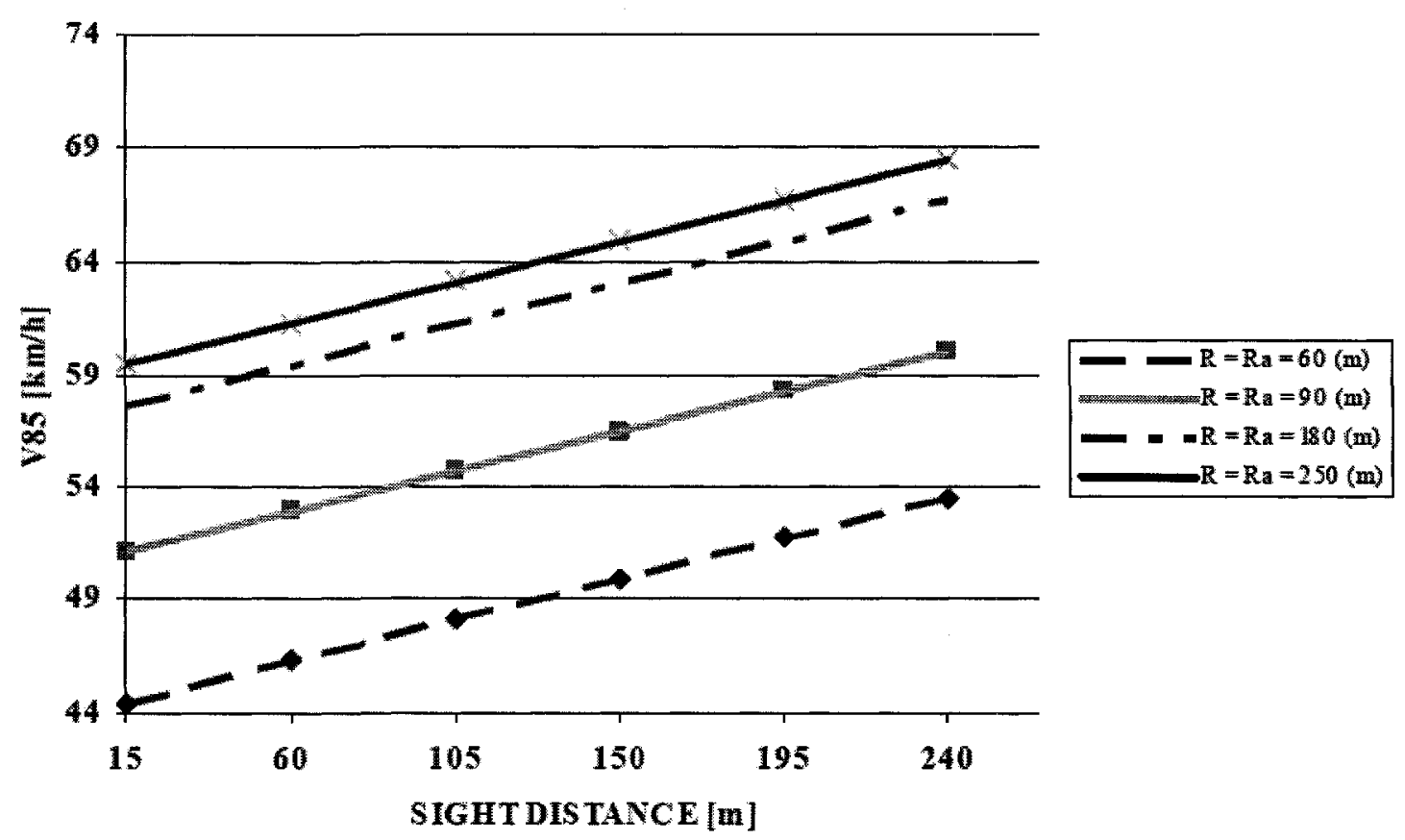

Figure 2-12: Relationship between AvSD and $85^{\text {th }}$ Percentile Speed (Discetti and Dell'Acqua 2008).

\subsection{Variations of Design Parameters}

Using the deterministic approach in the geometric design, there was always a need for single representative values that describe the driver population. This encouraged researchers to study the variation of several design parameters such as operating speed, acceleration/deceleration rate, and PR time. With the recent recognition of the role that the probabilistic approach may play in the design, little research studied appropriate probability density functions that best describe such variations. In this section, several parameters that serve the objectives of this research have been selected and presented. 


\subsubsection{Operating Speeds}

The operating speed, as defined in TAC (1999), is the speed that a driver would select to operate at a certain condition. Because driving conditions are not always ideal, operating speeds are most probably less than desired speeds. It was stated in both TAC (1999) and AASHTO (2004) that either a mean or $85^{\text {th }}$ percentile value is usually used to report vehicles speed. However, using such single values would insufficiently reflect how operating speeds are spread around these specific values.

There are numerous factors that would influence drivers' operating speeds such as the curve radius as studied by Andjus and Maletin (1998). Efforts were therefore devoted by the authors to quantify the drivers' behaviour on horizontal curves and to predict their operating speeds as a function of the curve radii. The study was conducted using nine horizontal curves on two-lane roads with radii ranging from $50 \mathrm{~m}$ to $750 \mathrm{~m}$. The study area was selected so that speeds would not be substantially affected by longitudinal grades. Using regression analysis, it was noticed that the $50^{\text {th }}$ percentile speed (V50), $85^{\text {th }}$ percentile speed (V85), and standard deviation increased with increasing curve radius, with relatively high correlation values.

Figure 2-13 shows the trend of such relations compared to other trends in past research. The figure also shows the two regression equations that link V85 and V50 to the curve radius. Moreover, the study presented a unique relationship that can be used to estimate the variation (i.e., standard deviation) as a function of V50, as presented in Figure 2-14. The figure suggests that the standard deviation increases linearly with 
increasing the average operating speed. In general, the relationships presented in this study are useful to predict the speed distribution on a curve with a known radius.

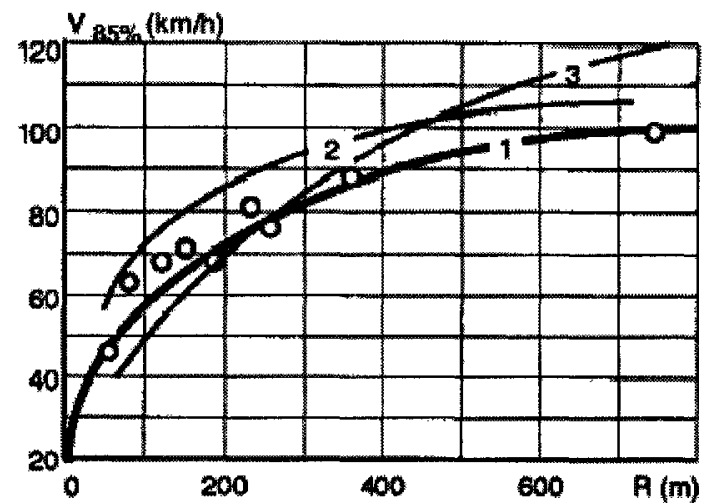

$1 V_{85 \%}=16.92 \ln R \cdot 14.49, r=0,975$

2 Lindemann, Ranft ( 8 )

3 YU standards ( 11)

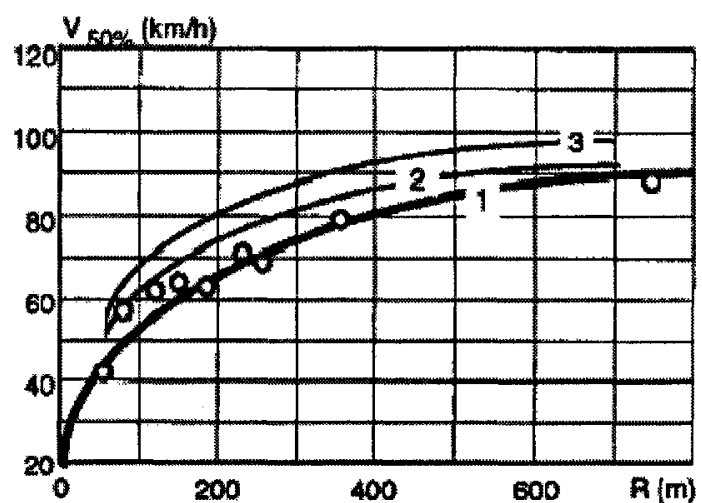

$1 V_{50 \%}=14.75 \ln R-11.69, r=0,969$

2 Lindemann, Rantt ( 8)

3 Gambard, Louah (6)

Figure 2-13: Relationship between Operating Speeds $\left(\mathrm{V}_{85}\right.$ and $\left.\mathrm{V}_{50}\right)$ and Curve Radius (Andjus and Maletin 1998).

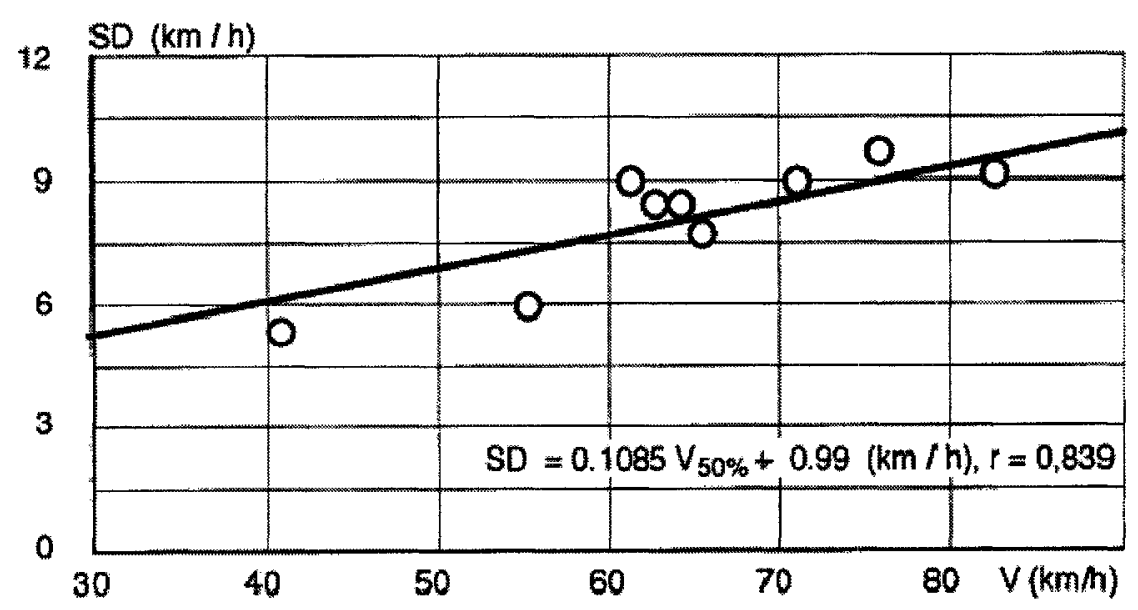

Figure 2-14: Relationship between Standard Deviation and V50 (Andjus and Maletin 1998). 
Furthermore, El Khoury and Hobeika (2006) suggested that the normal distribution could be acceptable to describe the variation of vehicles' speeds. To check this assumption, speeds were collected to assess the goodness of the normal distribution to fit these speeds. As shown in Figure 2-15, the speed data were fairly matched by a normal distribution.

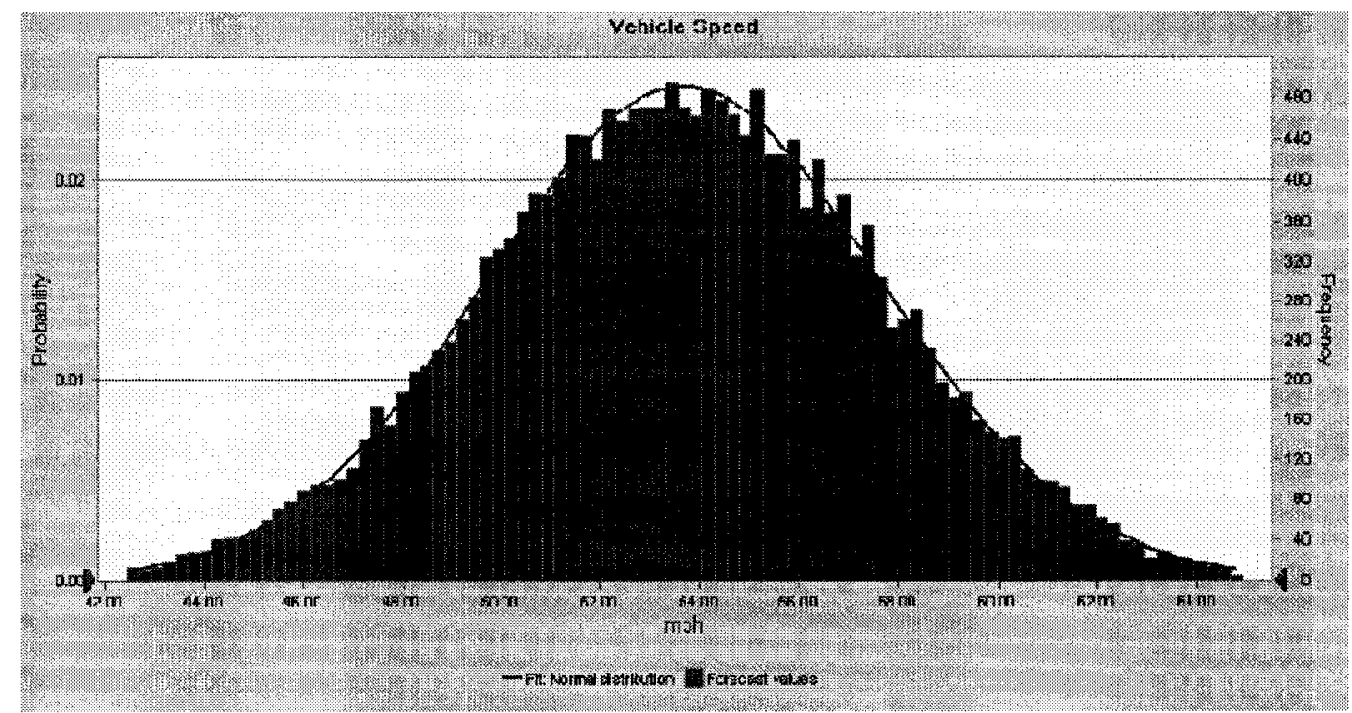

Figure 2-15: Analysis of Speed Distribution at $50 \mathrm{mph}$ Design Speed (E1 Khoury and Hobeika 2006).

\subsubsection{Acceleration and Deceleration Rate}

Acceptable deceleration rates $(a)$ for vehicles attempting to stop were discussed in AASHTO (2004) and were reported to be greater than $4.5 \mathrm{~m} / \mathrm{s}^{2}$. It was also stated that 90 $\%$ of all drivers tend to decelerate at a rate of, at least, $3.4 \mathrm{~m} / \mathrm{s}^{2}$. As mentioned, these values could keep the vehicle in the desired lane and help maintain steering control within the driver capabilities during the braking time on wet surfaces. It was also 
mentioned that most of current braking system capabilities and tire-pavement friction levels would guarantee deceleration rates more than $3.4 \mathrm{~m} / \mathrm{s}^{2}$.

In addition, Fambro et al. (2000c) studied the performance of braking and deceleration rates in both unexpected and anticipated stopping situations. Several factors were considered such as antilock braking systems (ABS), wet and dry pavement conditions, and road geometry effects to determine vehicle speeds, braking distances, and deceleration profiles. Using a Texas Transportation Institute (TTI) vehicle and drivers, 2000 braking manoeuvres were recorded. The reduction in required braking distances using the ABS system was obvious from the collected data, especially with increasing operating speeds. Table 2-2 summarizes the constant deceleration rates computed using the initial speed and the braking distance of individual braking attempts. Furthermore, the maximum deceleration rates ranged from 6.8 to $8.8 \mathrm{~m} / \mathrm{s}^{2}$ while average values were found to be $7.6 \mathrm{~m} / \mathrm{s}^{2}$ at $65 \mathrm{~km} / \mathrm{h}$ and $90 \mathrm{~km} / \mathrm{h}$ and $8.0 \mathrm{~m} / \mathrm{s}^{2}$ at $110 \mathrm{~km} / \mathrm{h}$.

Table 2-2: Summary of Constant Deceleration Rates for Different Pavement Conditions and Braking Systems (Adapted from Fambro et al. 2000c).

\begin{tabular}{cccccc}
\hline $\begin{array}{c}\text { Speed } \\
(\mathrm{km} / \mathrm{h})\end{array}$ & Pavement & ABS & $N$ & $\begin{array}{c}\text { Constant } \\
G\end{array}$ & $\begin{array}{c}\text { Standard } \\
\text { deviation }\end{array}$ \\
\hline \multirow{3}{*}{65} & \multirow{2}{*}{ Dry } & No & 191 & 0.60 & 0.122 \\
& & Yes & 176 & 0.62 & 0.134 \\
\cline { 2 - 6 } & \multirow{2}{*}{ Wet } & No & 203 & 0.49 & 0.067 \\
& & Yes & 186 & 0.54 & 0.071 \\
\hline \multirow{3}{*}{90} & \multirow{2}{*}{ Dry } & No & 216 & 0.65 & 0.135 \\
\cline { 2 - 6 } & \multirow{2}{*}{ Wet } & Yes & 203 & 0.71 & 0.163 \\
\hline & & No & 146 & 0.42 & 0.074 \\
& & Yes & 171 & 0.53 & 0.206 \\
\hline
\end{tabular}


Two other studies were further conducted by Fambro et al. (2000c) using (1) a single test vehicle and (2) personal vehicles of drivers. In these two studies, both maximum decelerating rates $\left(G_{\mathrm{x}}\right)$ and constant deceleration rates were obtained for unexpected and anticipated objects, as shown in Table 2-3 and Table 2-4, respectively. These two tables show that both mean values of maximum deceleration and standard deviations are greater in case of unexpected object compared to the expected-object case.

Table 2-3: Summary of Driver Deceleration to an Unexpected Object (Adapted from Fambro et al. 2000c).

\begin{tabular}{lcccccccc}
\cline { 4 - 8 } Study & & & \multicolumn{3}{c}{ Maximum Acceleration } & \multicolumn{2}{c}{ Constant Acceleration } \\
\hline Study 2 & No & Pavement & Geometry & $\begin{array}{c}\text { No. of } \\
\text { Test } \\
\text { Subjects }\end{array}$ & $\begin{array}{c}\text { Mean } \\
\text { Max }(\mathrm{g})\end{array}$ & $\begin{array}{c}\text { Standard } \\
\text { deviation } \\
(\mathrm{g})\end{array}$ & $\begin{array}{c}\text { Equivalent } \\
\text { Constant } \\
(\mathrm{g})\end{array}$ & $\begin{array}{c}\text { Standard } \\
\text { deviation } \\
(\mathrm{g})\end{array}$ \\
& Yes & Dry & Tangent & 6 & 0.91 & 0.08 & 0.62 & 0.07 \\
\hline Study 3 & No & Dry & Tangent & 7 & 0.91 & 0.14 & 0.63 & 0.08 \\
\hline
\end{tabular}

Table 2-4: Summary of Driver Deceleration to an Expected Object (Adapted from Fambro et al. 2000c).

\begin{tabular}{|c|c|c|c|c|c|c|c|c|}
\hline & & & & & & & \multirow{2}{*}{\multicolumn{2}{|c|}{ Constant Acceleration }} \\
\hline & & & & \multicolumn{3}{|c|}{ Maximum Acceleration } & & \\
\hline Study & ABS & Pavement & Geometry & $\begin{array}{c}\text { No. of } \\
\text { Test } \\
\text { Subjects }\end{array}$ & $\begin{array}{c}\text { Mean } \\
\operatorname{Max}(g)\end{array}$ & $\begin{array}{l}\text { Standard } \\
\text { deviation } \\
\text { (g) }\end{array}$ & $\begin{array}{l}\text { Equivalent } \\
\text { Constant } \\
\text { (g) }\end{array}$ & $\begin{array}{l}\text { Standard } \\
\text { deviation } \\
\text { (g) }\end{array}$ \\
\hline \multirow[t]{8}{*}{ Study 2} & No & Dry & Curve & 62 & 0.68 & 0.11 & 0.54 & 0.20 \\
\hline & & & Tangent & 54 & 0.70 & 0.13 & 0.53 & 0.08 \\
\hline & & Wet & Curve & 56 & 0.61 & 0.06 & 0.45 & 0.04 \\
\hline & & & Tangent & 50 & 0.63 & 0.06 & 0.49 & 0.04 \\
\hline & Yes & Dry & Curve & 48 & 0.73 & 0.18 & 0.54 & 0.11 \\
\hline & & & Tangent & 40 & 0.76 & 0.18 & 0.57 & 0.12 \\
\hline & & Wet & Curve & 51 & 0.68 & 0.11 & 0.51 & 0.09 \\
\hline & & & Tangent & 49 & 0.71 & 0.09 & 0.55 & 0.08 \\
\hline \multirow[t]{4}{*}{ Study 3} & No & Dry & Curve & 38 & 0.66 & 0.14 & 0.53 & 0.11 \\
\hline & & & Tangent & 38 & 0.68 & 0.14 & 0.54 & 0.11 \\
\hline & & Wet & Curve & 38 & 0.58 & 0.13 & 0.42 & 0.06 \\
\hline & & & Tangent & 43 & 0.63 & 0.09 & 0.45 & 0.06 \\
\hline
\end{tabular}




\subsubsection{Coefficient of Pavement Friction}

The coefficient of pavement friction $(f)$ varies widely as a function of several factors such as vehicle speed, tire type and condition, and pavement condition. As mentioned in TAC (1999), $f$ is considered as the most sensitive parameter to calculate SSDs. However, recommended friction values were referenced back to "tests carried out several decades ago" (TAC 1999). Table 2-5 shows the coefficient of friction values for wet pavements as presented in TAC (1999).

Table 2-5: Coefficient of Friction for Wet Pavements (Adapted from TAC 1999).

\begin{tabular}{ccc}
\hline $\begin{array}{c}\text { Design Speed } \\
(\mathrm{km} / \mathrm{h})\end{array}$ & $\begin{array}{c}\text { Operating } \\
\text { Speed }(\mathrm{km} / \mathrm{h})\end{array}$ & $\begin{array}{c}\text { Coefficient of Friction } \\
(f)\end{array}$ \\
\hline 30 & 30 & 0.40 \\
40 & 40 & 0.38 \\
50 & $47-50$ & 0.35 \\
60 & $55-60$ & 0.33 \\
70 & $63-70$ & 0.31 \\
80 & $70-80$ & 0.30 \\
90 & $77-90$ & 0.30 \\
100 & $85-100$ & 0.29 \\
110 & $91-110$ & 0.28 \\
120 & $98-120$ & 0.28 \\
130 & $105-130$ & 0.28 \\
\hline
\end{tabular}

Echaveguren et al. (2005) studied the equilibrium between the demanded and supplied pavement friction. The supplied friction was mentioned to vary based on the tire-pavement interaction and operating speed. It was also mentioned that the friction decreases exponentially as speed increases. A model was then developed to predict the supplied friction as a function of speed, texture, and skid resistance (FRS). The normal distribution was stated to fit the pavement friction in many instances in the literature. In addition, a study done by Cafiso (2000), and using data from Italian highways, concluded 
the gamma distribution as the best fitting one. Moreover, the authors observed that the variance of pavement friction decreases as speed increases.

\subsubsection{Perception-Reaction Time}

The perception and reaction time $(P R)$ is the time that drivers take to detect an object and initiate the execution of decided actions. This time, as stated in AASHTO (2004), is also called brake reaction time if the decided action was stopping. The PR time changes according to the degree of complexity of a specific situation. The more complex a specific situation, the greater the time needed. In this regard, Brydia and Pietrucha (1994) discussed the influence of PR time on case III intersection SD, in which stop control exists on a minor road. The objective was to help determine whether or not very exact PR time is essential to appropriately design intersections. The study also assessed different values to be used as a PR time in calculating SSD at intersections based on previous work of appropriate PR times. It was concluded that, although several values are recommended, no final agreement on what values should be used for various design purposes.

In addition, a comprehensive study by Fambro et al. (1998) addressed the driver perception-brake response in SSD situations. As mentioned earlier, the two parts that form the required SSD are the brake reaction distance and braking distance. The first part is a function of the vehicle speed and driver PR time while this time is the summation of brake reaction time and perception time. It was mentioned in this study that the brake reaction time was held at 1 sec since 1940 when first assumed by AASHO (1940). In addition, the total PR time was assumed to range from $2 \mathrm{sec}$ to $3 \mathrm{sec}$, as a function of the 
design speed. Later, and according to AASHO (1954), this total time was suggested to be $2.5 \mathrm{sec}$.

In the same study, Fambro et al. (1998) summarized the PR time that was documented in several studies as shown in Table 2-6. Two types were displayed in this table including unexpected and anticipated values. For the first type, and more specifically the studies under the covert title, PR times were due to unexpected signals and had average and standard deviation of 1.27 and $0.66 \mathrm{sec}$, respectively. It was stated that the consistency and large sample sizes that were used in those studies suggest a good estimate of the measured times for responding to a yellow traffic signal. A close average but lower value of the standard deviation of 1.28 and 0.20 , respectively, was further found in response to other unexpected objects in other studies. As for the second type, several studies showed that the average and standard deviation of 0.56 and 0.10 were observed in studies using simulators while 0.73 and 0.16 were found in real-world studies. These values were expectedly less than those of the first part since drivers had an early alert to such objects.

Moreover, Fambro et al. (1998) provided additional information on braking performance by conducting four field studies using an in-vehicle instrumentation package to measure driver PR response times, braking distances, and decelerations to unexpected and anticipated stops. Out of these four studies, the first study was carried out with a single vehicle on a closed course using more than 2000 braking manoeuvres while the second and third studies utilized single and multiple test vehicles, respectively, on the same course and using more than 1000 braking manoeuvres. 
Table 2-6: Summary of Surprise and Anticipated Perception-Brake Reaction Time Studies (Adapted from Fambro et al. 1998).

\begin{tabular}{|c|c|c|c|c|c|}
\hline & $\begin{array}{c}\text { No. of } \\
\text { observations }\end{array}$ & Ages & Mean & $\begin{array}{l}\text { Standard } \\
\text { deviation }\end{array}$ & Stimulus \\
\hline \multicolumn{6}{|l|}{ Covert: } \\
\hline Gazis et al. (12) & 87 & Mix & 1.12 & -- & Unexpected Signal \\
\hline Sivak et al. (13) & 1644 & Mix & 1.21 & 0.63 & Unexpected Signal \\
\hline Wortman and Matthias (14) & 839 & Mix & 1.3 & 0.6 & Unexpected Signal \\
\hline Chang et al. (15) & 579 & Mix & 1.3 & 0.74 & Unexpected Signal \\
\hline Mean Estimates & & & 1.27 & 0.66 & \\
\hline \multicolumn{6}{|l|}{ Surprise: } \\
\hline Olson and Sivak (16) & 49 & Young & 1.1 & 0.15 & Unexpected Object \\
\hline Olson and Sivak (16) & 15 & Old & 1.06 & 0.1 & Unexpected Object \\
\hline Sivak M. (17) & 1644 & Mix & 1.21 & 0.63 & Unexpected Brake \\
\hline Lerner et al. (18) & 56 & Mix & 1.5 & 0.4 & Light, Unexpected object \\
\hline Mean Estimates & & & 1.28 & 0.2 & \\
\hline \multicolumn{6}{|c|}{ Study Condition: Anticipated (Alerted Driver) } \\
\hline \multicolumn{6}{|l|}{ Driver simulator: } \\
\hline Rackett et al. (19) & 114 & Mix & 1.31 & 0.11 & Onset Red \\
\hline Retchin et al. (20) & 61 & Old & 0.66 & 0.66 & Bumpa-Tel Test \\
\hline Retchin et al. (20) & 38 & Old & 0.84 & 0.1 & Bumpa-Tel Test \\
\hline Cation et al. (21) & 104 & Mix & 0.43 & 0.1 & Onset Red Light \\
\hline Mean Estimates & & & & 0.1 & \\
\hline \multicolumn{6}{|l|}{ Behind the wheel: } \\
\hline Olson and Sivak (16) & 49 & Young & 0.72 & 0.11 & Anticipated Object \\
\hline Olson and Sivak (16) & 15 & Old & 0.73 & 0.1 & Anticipated Object \\
\hline Johansson, Rumar (9) & 321 & Mix & 0.75 & 0.28 & Anticipated Horn \\
\hline Mean Estimates & & & 0.73 & 0.16 & \\
\hline
\end{tabular}

The fourth and last study was an open-road study to measure driver braking performance to an unexpected object scenario. In addition, Table 2-7 summarizes the results of the four studies being divided into three parts; namely (1) perception brakeresponse time to an unexpected object, (2) perception brake-response time to an expected object, and (3) baseline perception-response time observations. The first part indicates that, for unexpected-object situations, the mean values ranged from 0.82 to $1.14 \mathrm{sec}$ while 
the standard deviations ranged from 0.159 to 0.353 . For the second part, ranges of 0.48 to 0.67 and 0.078 to 0.345 were corresponding to means and standard deviations, respectively. These values again were less than those of unexpected objects. It was also concluded that the overall mean perception brake-response time to an unexpected object is about $1.1 \mathrm{sec}$ under controlled and open-road conditions.

Table 2-7: Summary of Perception-Brake Response Times (Adapted from Fambro et al. 1998).

\begin{tabular}{|c|c|c|c|c|c|}
\hline Study & \multicolumn{2}{|c|}{ Age } & $\begin{array}{l}\text { No. of Test } \\
\text { Subjects }\end{array}$ & $\begin{array}{c}\text { Mean PBRT } \\
(\mathrm{sec})\end{array}$ & $\begin{array}{c}\text { Standard } \\
\text { deviation (sec) }\end{array}$ \\
\hline \multicolumn{6}{|c|}{ Unexpected Object: } \\
\hline \multirow[t]{2}{*}{ Study 2} & \multicolumn{2}{|c|}{ Older } & 12 & 0.82 & 0.159 \\
\hline & \multicolumn{2}{|c|}{ Younger } & 10 & 0.82 & 0.203 \\
\hline \multirow[t]{2}{*}{ Study 2} & \multicolumn{2}{|c|}{ Older } & 7 & 0.14 & 0.353 \\
\hline & \multicolumn{2}{|c|}{ Younger } & 3 & 0.93 & 0.191 \\
\hline \multirow[t]{2}{*}{ Study 2} & \multicolumn{2}{|c|}{ Older } & 5 & 1.06 & 0.222 \\
\hline & \multicolumn{2}{|c|}{ Younger } & 6 & 1.14 & 0.204 \\
\hline \multicolumn{6}{|c|}{ Expected Object: } \\
\hline \multirow[t]{4}{*}{ Sudy 2} & \multirow[t]{2}{*}{ Older } & Female & 7 & 0.66 & 0.216 \\
\hline & & Male & 7 & 0.65 & 0.228 \\
\hline & \multirow[t]{2}{*}{ Younger } & Female & 6 & 0.57 & 0.167 \\
\hline & & Male & 6 & 0.48 & 0.088 \\
\hline \multirow[t]{4}{*}{ Study 3} & \multirow[t]{2}{*}{ Older } & Female & 5 & 0.67 & 0.252 \\
\hline & & Male & 3 & 0.65 & 0.345 \\
\hline & \multirow[t]{2}{*}{ Younger } & Female & 2 & 0.49 & 0.168 \\
\hline & & Male & 1 & 0.55 & 0.078 \\
\hline \multicolumn{6}{|c|}{ Baseline Observation: } \\
\hline \multirow[t]{4}{*}{ Study 2} & \multirow[t]{2}{*}{ Older } & Female & 7 & 0.5 & 0.09 \\
\hline & & Male & 7 & 0.47 & 0.06 \\
\hline & \multirow[t]{2}{*}{ Younger } & Female & 6 & 0.47 & 0.13 \\
\hline & & Male & 6 & 0.42 & 0.08 \\
\hline \multirow[t]{4}{*}{ Study 3} & \multirow[t]{2}{*}{ Older } & Female & 5 & 0.47 & 0.08 \\
\hline & & Male & 3 & 0.44 & 0.1 \\
\hline & \multirow[t]{2}{*}{ Younger } & Female & 2 & 0.39 & 0.06 \\
\hline & & Male & 1 & 0.48 & 0.04 \\
\hline \multirow[t]{2}{*}{ Control } & \multirow[t]{2}{*}{ Older } & Female & 10 & 0.52 & 0.19 \\
\hline & & Male & 8 & 0.43 & 0.14 \\
\hline
\end{tabular}


In addition, most drivers were found capable of responding to a SSD situation in 2 $\mathrm{sec}$, which is less than the $2.5 \mathrm{sec}$ recommended by AASHTO (2004). It should be noted that these studies presented above did not consider the effect of the traffic on the deceleration behaviour. A technical report was prepared by General Motors Corporation and Delphi-Delco Electronic Systems (2002) to design and evaluate the driver-vehicle interface considering different human factors. It was stated in this study that a driver reaction while decelerating is strongly linked to the time headway available ahead of his/her vehicle. In cases where drivers have large time-headways (e.g., greater than 3 seconds), decelerations might be postponed several seconds or low deceleration rates might be favoured. On the other hand, in cases where time headways are relatively small (e.g., less than 0.5 second), drivers' responses might be almost immediate in order to avoid a collision.

\subsubsection{Driver Eye, Object, and Headlight Height}

In highway geometric design, both driver eye and object heights are important variables and would significantly influence the design of several highway elements such as side slopes and lengths of vertical curves. It was stated in TAC (1999) that $99 \%$ of the total vehicle population has a driver eye height of at least $1.05 \mathrm{~m}$ above road surface when passenger cars are assumed to be taken as the critical design vehicle. Moreover, a value of $1.8 \mathrm{~m}$ was considered as a suitable eye height for buses and single unit vehicles. For large trucks and truck trailer combinations, a range of $1.9 \mathrm{~m}$ to $2.4 \mathrm{~m}$ with average of $2.1 \mathrm{~m}$ was recommended for design purposes. In AASHTO (2004), values were slightly higher than those recommended in TAC (1999) as average driver eye height was 
estimated to be $1.08 \mathrm{~m}$ above roadway surface for AvSD calculations for passenger vehicles. As for large trucks, this value ranges from $1.8 \mathrm{~m}$ up to $2.4 \mathrm{~m}$ with a recommended value of $2.33 \mathrm{~m}$ above roadway surface.

Harwood et al. (1989) reported that previous research suggested truck driver eye heights between 71.5 inch $(1.82 \mathrm{~m})$ and 112.5 inches $(2.86 \mathrm{~m})$. In addition, an average driver eye height for a conventional tractor was estimated to be 93 inch $(2.36 \mathrm{~m})$. In addition, Fitzpatrick et al. (1998) evaluated the heights of driver eye, headlight, taillight, and vehicles as important elements in the determination of AvSD. The main objectives of the study were to review previous relevant studies, collect real-world data, and finally recommend appropriate heights to be used in the geometric design. It was stated that design values of driver eye height decreased from $1,676 \mathrm{~mm}$ in $1920 \mathrm{~s}$ to 1,070 or even lower after the new revolution of vehicle design, as vehicles became smaller and more compact to reduce fuel consumptions. Table 2-8 summarizes recent driver eye heights since 1957 as reported by Fitzpatrick et al. (1998).

On the other hand, it was stated in TAC (1999) that objects' heights have even more impact on AvSD than driver eye height. As documented, the values of such objects would range from zero (e.g., pavement markings or road washouts) up to $1.67 \mathrm{~m}$. However, a vehicle taillight height of 0.38 was said to represent a conservative value to represent an object along the carriageway for SSDs calculations. It should be noted that no suggestions for appropriate distributions of such heights were found in TAC (1999). 
Table 2-8: Summary of Driver Eye Height Studies (Adapted from Fitzpatrick et al. 1998).

\begin{tabular}{|c|c|c|c|c|c|c|}
\hline Study & $\begin{array}{l}\text { Stonex } \\
\text { (3) }\end{array}$ & $\begin{array}{l}\text { Lee } \\
(6)\end{array}$ & $\begin{array}{l}\text { Boyd et } \\
\text { al. (7) }\end{array}$ & $\begin{array}{c}\text { Cunagin and } \\
\text { Abraharasor } \\
\text { (8) }\end{array}$ & $\begin{array}{l}\text { Haslegrave } \\
\text { (9) }\end{array}$ & $\begin{array}{c}\text { Barker } \\
(10)\end{array}$ \\
\hline Mean $(\mathrm{mm})$ & 1295 & 1289 & 1125 & NA & 1145 & 1130 \\
\hline height 15th percentile & 1242 & 1204 & 1054 & 1067 & 1092 & 1070 \\
\hline Vehicle types & $\mathrm{PC}$ & $\mathrm{PC}$ & $\mathrm{PC}$ & $\mathrm{PC}, \mathrm{PU}$ & PC,PU,V & PC,PU \\
\hline Data points & 1967 & 761 & 195 & 1478 & 825 & 1124 \\
\hline Year & 1957 & 1960 & 1978 & 1979 & 1979 & 1987 \\
\hline Country & U.S. & U.S. & U.S. & U.S. & U.K. & Australia \\
\hline
\end{tabular}

$\mathrm{PC}=$ passenger car; $\mathrm{PU}=$ pickup truck; and $\mathrm{V}=$ van

It was mentioned by Fitzpatrick et al. (1998) that standard 108 of the Federal Motor Vehicle Safety Standards specifies boundaries for lighting equipment on motor vehicles. For example, headlight heights were recommended to be between $560 \mathrm{~mm}$ and $1,370 \mathrm{~mm}$ besides a minimum of $380 \mathrm{~mm}$ and a maximum of 1,830 for the taillight. Moreover, Fitzpatrick et al. (1998) collected data from seven sites in four states; Washington, Illinois, Texas, and Virginia. The data were obtained by using two cameras and a calibration vehicle with known dimensions.

Table 2-9 summarizes the sample size, mean value, and other percentile values that were measured for three vehicle types including passenger cars, multipurpose vehicles, and heavy trucks. It was found that $97 \%$ of the 1667 passenger cars, multipurpose vehicles, and large trucks had driver eye heights greater than the $1070 \mathrm{~mm}$ recommended by AASHTO (1994). It was also found that, out of the 1318 passenger car headlight heights, only 10 were below the $560-\mathrm{mm}$ requirement of Standard 108. In addition, no multipurpose vehicles or large trucks were below this standard. 
Table 2-9: Driver Eye, Headlight, Taillight, and Vehicle Heights for Different Vehicle Types (Adapted from Fitzpatrick et al. 1998).

\begin{tabular}{lccc}
\cline { 2 - 4 } & Passenger car & Multipurpose vehicle & Heavy trucks \\
\hline Driver eye height & & & \\
\hline Sample size & 875 & 629 & 163 \\
Mean (mm) & 1149 & 1482 & 2447 \\
5th percentile (mm) & 1060 & 1264 & 2304 \\
10th percentile (mm) & 1082 & 1306 & 2329 \\
15th percentile (mm) & 1094 & 1313 & 2341 \\
\hline Headlight height & & & 337 \\
\hline Sample size & 1318 & 992 & 1121 \\
Mean (mm) & 649 & 842 & 972 \\
5th percentile (mm) & 590 & 691 & 1008 \\
10th percentile (mm) & 602 & 713 & 1022 \\
15th percentile (mm) & 608 & 728 & 260 \\
\hline Taillight height & & & 1058 \\
\hline Sample size & 858 & 534 & 719 \\
Mean (mm) & 726 & 963 & 908 \\
5th percentile (mm) & 616 & 780 & 953 \\
10th percentile $(\mathrm{mm})$ & 642 & 818 & \\
15th percentile (mm) & 660 & 839 & \\
\hline
\end{tabular}

As for the 1652 taillight heights, none of the vehicles was observed below the standard value of $380 \mathrm{~mm}$. Finally, a driver eye height of $1080 \mathrm{~mm}$, a headlight height of $660 \mathrm{~mm}$, and a taillight height of $600 \mathrm{~mm}$ were recommended in this study.

\subsection{Risk Assessment and Reliability Analysis in Transportation}

The risk assessment and probability of collisions have been of interest to highway researchers over the last few years. Several methodologies were developed to evaluate the likelihood of a collision under certain driving conditions and road characteristics. These methodologies relied in many instances on the probability theory and existing collision records in attempts to configure possible trends and patterns of the collision occurrence. In this section, different studies will be presented to show the application of 
different techniques to conduct risk assessment including the reliability analysis approach.

The study done by Blasco et al. (2003) addressed the probability of collisions with respect to the occurrence of other ones on a transportation network. The study used 8year collision data of a public bus company in a large Spanish city. The authors struggled to answer the question of whether bus drivers become involved in traffic collisions on a regular basis with a certain pattern or in a grouped manner. The hypothesis in the study was that the probability of having a collision is a function of the period of time between two successive collisions for the same driver. It was mentioned that the probability of a collision decreases with increasing the time interval between a collision and the previous one for the same driver. The reason for this phenomenon, as believed by the authors, is that collisions occur sequentially for the same driver. It was concluded that collisions in general tend to occur closer in time than can be expected by chance. In addition, the occurrence of a collision increases the probability of the following collision.

The relationship between age and sex of drivers and the risk of causing vehicle collisions were also addressed by Claret et al. (2003). The study included 220,284 collisions registered from 1990 to 1999 in the Spanish Direction General de Trafico (DGT) traffic crash database. Only collisions that had one responsible driver while the other is non-responsible were used in the study. For age-related comparisons, odds ratios were calculated for each age category, using men age range of 40 to 44 years as the reference. For sex-related comparisons, male drivers were the comparison reference. It was concluded in this study that the lowest risk values for men were found for the 25-49- 
year-old age group and increased exponentially after the age of 50 years. For women, the lowest risk values were found for 25-44-years-old age and also increased with age. It could be stated that, according to the data that were used in the study, age and sex might play a significant role to determine the probability of having a collision (see Figure 2-16).

Reliability analysis was also one of the crucial tools that were used to conduct a risk assessment of a transportation system. Several studies handled the effectiveness of the reliability approach to analyze existing and new designs of different road elements. The application of reliability analysis in transportation engineering was initiated by BenAkiva et al. (1985).

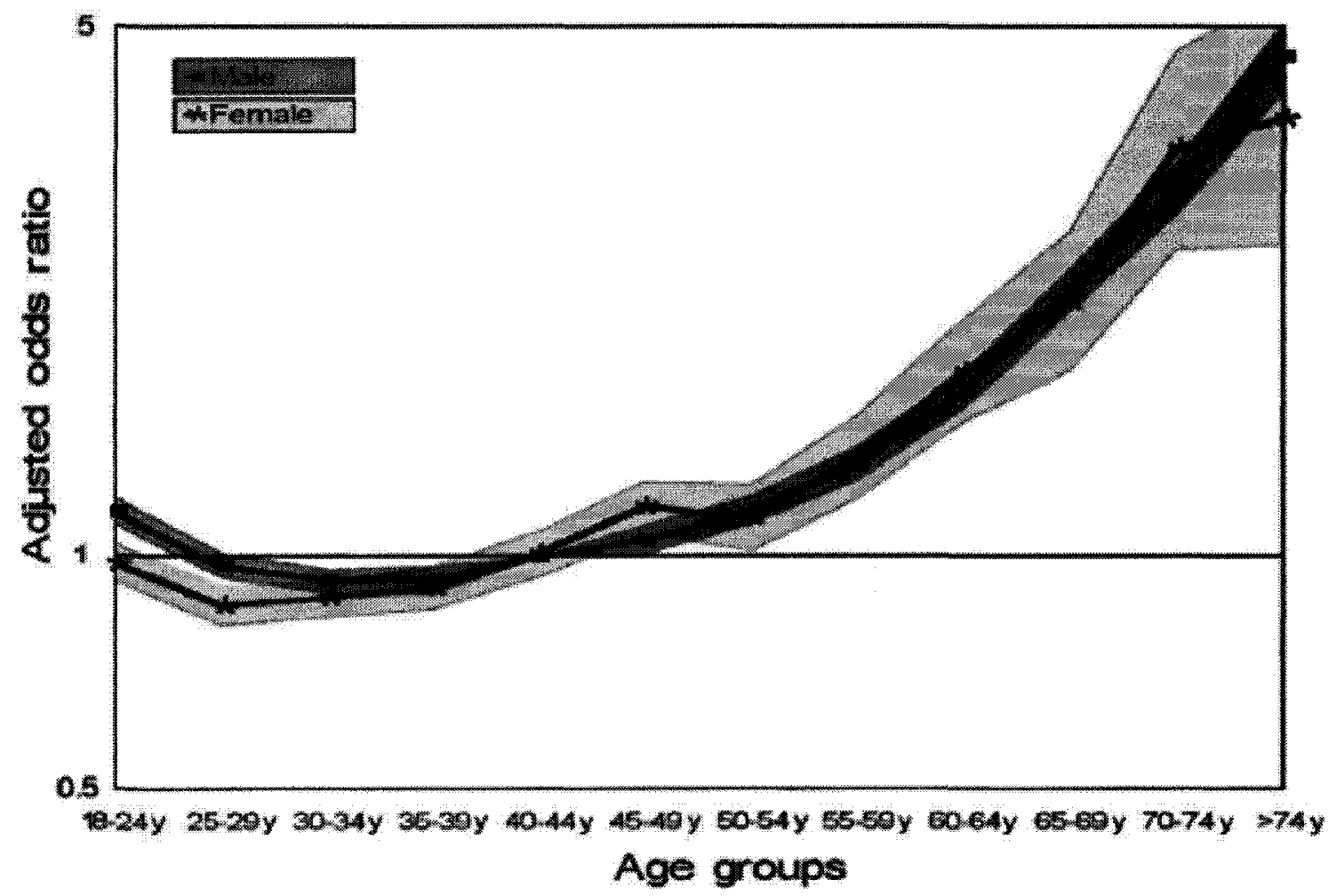

Figure 2-16: The Effect of Age and Sex on the risk of causing a vehicle collision (Claret et al. 2003). 
As stated, "the geometric design standards widely used in highways design are deterministic in character and thus ignore important probabilistic factors of highways operations." (Ben-Akiva et al. 1985). For this reason, it was recommended to change current design standard concept by a design process, which uses all available data to select the most appropriate design criteria for a specific situation. The new approach that was proposed by Ben-Akiva et al. (1985) depends on using all possible parameters being represented by different distributions. These distributions were recommended to be measured or estimated reasonably from previous studies' data. Two examples were further used to discuss the new approach including (1) calculating a vertical curve radius in terms of speed, friction value, driver perception-reaction time, driver eye height, and obstacle height, and (2) calculating the length of climbing lane in terms of the grade and length of uphill highway section and speeds of light vehicles and trucks.

Later, Easa (1994) used the probabilistic approach to evaluate SDs at railroad grade crossings. It was emphasized that the probabilistic approach in geometric design has many advantages over the deterministic approach that is still being used in current design guides. Two cases were studied in this research; (1) RqSD along a highway and a railroad for a moving vehicle and (2) RqSD along a railroad for a stopping vehicle. The main objective of the study was to provide safer operations at railroad grade crossings. The probabilistic method in this study was based on the advanced first-order secondmoment of reliability analysis. The method helps obtain SDs along both the road and railroad that result in a specific system probability of failure. Sensitivity analysis was also conducted to address the change in the reliability index when each variable changes by 10 
$\%$. The method is generally powerful; however, as mentioned by the author, the validation of the proposed method with actual data was not done due to lack of such data.

In addition, Navin and Zheng (1998) used the tire-road-vehicle interaction to estimate a drivers' relative level of safety with the expert driver being the reference. Navin and Zheng (1998) said that highway design parameters are uncertain and vary within known bounds that can be obtained by surveys. These variables yield distributions for both supplies and demands. If the distributions of demands and supplies of a specific road feature intersect, the resulting overlapping area, referred to as non-compliance, is the area where the demand is expected to exceed the supply. It was emphasized that the existence of this area does not necessitate the occurrence of highway collisions. As mentioned, the reliability-based design should aim at reducing these overlapping areas.

Navin and Zheng (1998) also made a comparison between race cars and road cars to distinguish between driver-vehicle-tire-capacity and operational limits of road cars. The rationale of such comparison is that racers try to achieve maximum performance from the road-tire-driver combination in order to win their races. Therefore, race cars were considered the theoretically limiting supply of the vehicle for any given operating conditions. The difference between the performance of a race car and the performance of a passenger car, referred to as reliability index $(\beta)$, was taken by the authors as a measure of safety. However, the difference between maximum technological capabilities of race vehicles and the practical design that account for passenger safety and comfort was highlighted. 
In more detail, the method presented in Navin and Zheng (1998)'s study handled three issues; (1) horizontal SD, (2) turning radius, and (3) vertical SD. In addition, there were four driving scenarios including AASHTO and TAC design driver, expert (race car) driver at maximum speed, normal driver at a comfortable speed, and normal driver at maximum speed. The study was conducted on a 100-km two-lane segment on Highway 99 located in British Columbia. The values of $\beta$ were estimated at $20 \mathrm{~m}$ intervals on curves and subsequently the overall reliability profile was finally drawn. Arbitrarily, acceptable design was assumed for $\beta$ values more than 3.5. A total of ten locations were found dangerous based on the assumptions of this study. However, it was mentioned that the authors could not study the collisions at these locations since collision records did not have accurate location codes.

Moreover, Sigbjornsson and Snaebjornsson (1998) developed a general probabilistic model to assess road vehicle collisions in windy environments. Reliability analysis was the basis of the proposed method, in which a performance function was first set and suitable stability criteria were then selected to define limit states of a safe performance. It was mentioned that high-sided vehicles such as buses are exposed to wind forces especially at locations where topographical features of the landscape magnify the wind effect. As stated, most of the forces influencing a moving vehicle such as wind velocity and direction, vehicle speed and direction, mass of the vehicle and location of centre of gravity, friction coefficient, road geometry and spatial curvature of the vehicle path are all uncertain, with different degrees of uncertainty. 
These stochastic variables were called basic variables while other geometric and material properties of vehicles were treated as deterministic variables. The limit states of the safe performance were linked to loss of vehicle stability, which would lead to side slip or even overturning of moving vehicles. The proposed method was applied in a real case study when a bus with 41 passengers went off the road in a high cross wind and rolled over. Probability of side slip was computed for a given mean surface friction, mean wind velocity, and mean vehicle speed. In addition, relative contribution of basic variables to the collision were conducted and plotted on graphs. It was generally stated that "theory of reliability ... can be helpful in post-evaluation of accidents and to improve the design of roads and highways" (Sigbjornsson and Snaebjornsson 1998).

Easa (2000) recommended using the reliability analysis in designing intersection sight distances (ISD); for both Cases $I$ and $I I$ as defined in AASHTO design guide. It was mentioned that the proposed method replaces the common uses of extreme values (high percentile) of design variables such as design speed, perception and reaction time, and friction coefficient with the moments (mean and variance) of probability distributions of all these random variables. The method would also be beneficial as it accommodates correlations among such variables. The method was built specifically on the first-order probabilistic analysis as one of the simplest reliability analysis techniques. As stated, one of the advantages of this type of analysis is that it requires no assumption about the types of variables' distributions.

Easa (2000) defined a failure of a specific approach as the case when its AvSD does not meet its RqSD. Another term, referred to as a system failure, was also used to 
indicate failures in two approaches forming a sight triangle at a specific intersection. As assumed by Easa (2000), a collision is expected to take place only when a system failure occurs in an intersection. The usefulness of the proposed method is that it provides infinite number of solutions with infinite number of failure probabilities. Comparisons between recommended values in AASHTO design guide (as a deterministic approach) and resulting values from the proposed method have revealed the flexibility of the second. However, real data were stated to be important for validation.

The margin of safety of an existing curve was estimated by Echaveguren et al. (2005) using the difference between the design speed and speed limit, which can be obtained from the demand and supply equilibrium of the pavement friction. A failure was assumed if the available friction was not enough to compensate the demand friction. Several actions were also suggested to reduce the probability of failure including modification of the curve geometry, reduction of operating speeds, and improvement of the surface friction.

Recently, a study by El Khoury and Hobeika (2006) used the reliability analysis to estimate the PSD requirements. The main objective of this study was to derive a PSD distribution depending on variations in other contributing parameters. Both analytical technique and Monte Carlo simulation were used to achieve the study objectives at 50mph design speed. The study revised a PSD model that was first suggested by Glennon (1998) in order to account for a variable PR time. In addition, two different variables were used to represent the clearance distance between passing and impeding vehicles. The distributions of the contributing factors are listed as follows: 
- Traffic speed: truncated normal / known

- Speed difference between passing and impeding vehicle: log normal / known

- Driver perception and reaction time: log normal / known

- Clearance distances required to complete a passing manoeuvre: uniform / assigned

- Minimum gap distances required to abort a passing manoeuvre: uniform / assigned

- Deceleration rates: truncated normal / assigned

- Percent of vehicles in each vehicle class: discrete / known

- Vehicle lengths classified by vehicle class: discrete / known

The term known or assigned was to indicate whether the authors conducted their own study or took advantage of recent studies, respectively, in order to obtain suitable distribution type for individual random variables. Using Mathematica software to conduct the analytical calculations and using Crystal Ball package for Monte Carlo simulations, the authors were able to obtain and assess the reliability index of current PSD standards presented in AASHTO's green book and MUTCD as well as Glennon's design values. It was mentioned that MUTCD and Glennon's design values were close to the mean of PSD distribution in this study while PSD values in AASHTO were overestimated. 


\section{CHAPTER 3}

\section{ReLIABILITY-BASED DESIGN APPROACH}

This chapter covers the methodology that will be followed in achieving the thesis objectives. Section 3.1 presents a general discussion on the reliability analysis and includes four subsections; Section 3.1.1 discusses the main concept, Section 3.1.2 displays different measures of a system reliability, Section 3.1.3 summarizes different common types of reliability analysis, and Section 3.1.4 discusses the difference between reliability applications in geometric design and its applications in other design branches. In addition, Section 3.2 presents a proposed geometric design framework based on the probability concept. The contribution of the research is further presented in Section 3.3. To facilitate the application of the proposed work, a tool was developed and programmed as described in Section 3.4. A summary is finally presented in Section 3.5.

\subsection{Reliability Analysis}

Reliability analysis has gained a great popularity in several engineering branches, especially in structural and geotechnical. In transportation engineering, the application of reliability is relatively limited compared to other engineering branches. However, as shown earlier in 2.5 , recent research has shown the applicability and potential usefulness of such analysis in several areas of study such as signal timing and sight distance at railroad grade crossing. 


\subsubsection{General Concept}

In generic terms, reliability analysis deals with uncertainties of the supply $(S)$ and demand $(D)$ of a specific system. It assesses the system's ability to accommodate the demand against the capacity of that system. By definition, the reliability of a certain system is the probability that such system will succeed to perform a specific task whereas the risk can be evaluated by the probability of failure. The general concept of the reliability analysis relies on two distributions representing both $S$ and $D$. The two distributions are usually defined in terms of other design parameters that vary with time, location, and prevailing conditions. These parameters' variation might place a great difficulty in quantifying the system ability to perform properly.

In the context of transportation engineering, $S$ could be the strength of the pavement layers and paving materials whereas $D$ would be the number of load repetitions on a specific road segment. In such example, there are uncertainties in both $S$ and $D$ that suggest decisions to be built on a specific and pre-determined confidence level. This strategy was realized in the structure design of asphalt pavements in the AASHTO design guide by involving a reliability index and an overall standard deviation in the design process. However, no indication of reliability applications was yet observed in the geometric design methodology. The reliability analysis can similarly handle uncertainties and variations of several design parameters in geometric design such as sight distances, driver characteristics, and vehicle capabilities. 


\subsubsection{Measures of Reliability}

Having two distributions for $S$ and $D$, there are different ways of assessing a system's reliability. The traditional and most conventional way is the probability of failure that is often used in almost all engineering branches. It should be noted, however, that the term failure does not suite the expected risk from an unsuitable geometric design. By definition, a failure means a loss of ability to function normally and will immediately occur when the demand reaches the system's capacity. While such failure is usually observed as permanent and non-reversible in other engineering branches, a failure of a transportation facility might be reflected as a collision or a traffic queue that lasts for a certain time. Therefore, the term hazard is considered more appropriate compared to failure in transportation context.

Conceptually, the probability of hazard $(\mathrm{POH})$ takes values greater than zero when both $S$ and $D$ distributions overlap with a possibility of having $S$ less than $D$. The $\mathrm{POH}$ can then be used as a measure of risk that the system might fail under certain loading conditions. As shown in Figure 3-1, the $\mathrm{POH}$ at a given value of $y$ is the product of the density function of the supply $\left[f_{\mathrm{s}}(y)\right]$ by the probability $\left[1-F_{\mathrm{D}}(y)\right]$ that the demand is exceeding this $y$ value. By taking the integration from zero to infinity, one can estimate the overall $\mathrm{POH}$ as follows:

$$
P O H=\int_{0}^{\infty} f_{S}(y)\left[1-F_{D}(y)\right] d y
$$




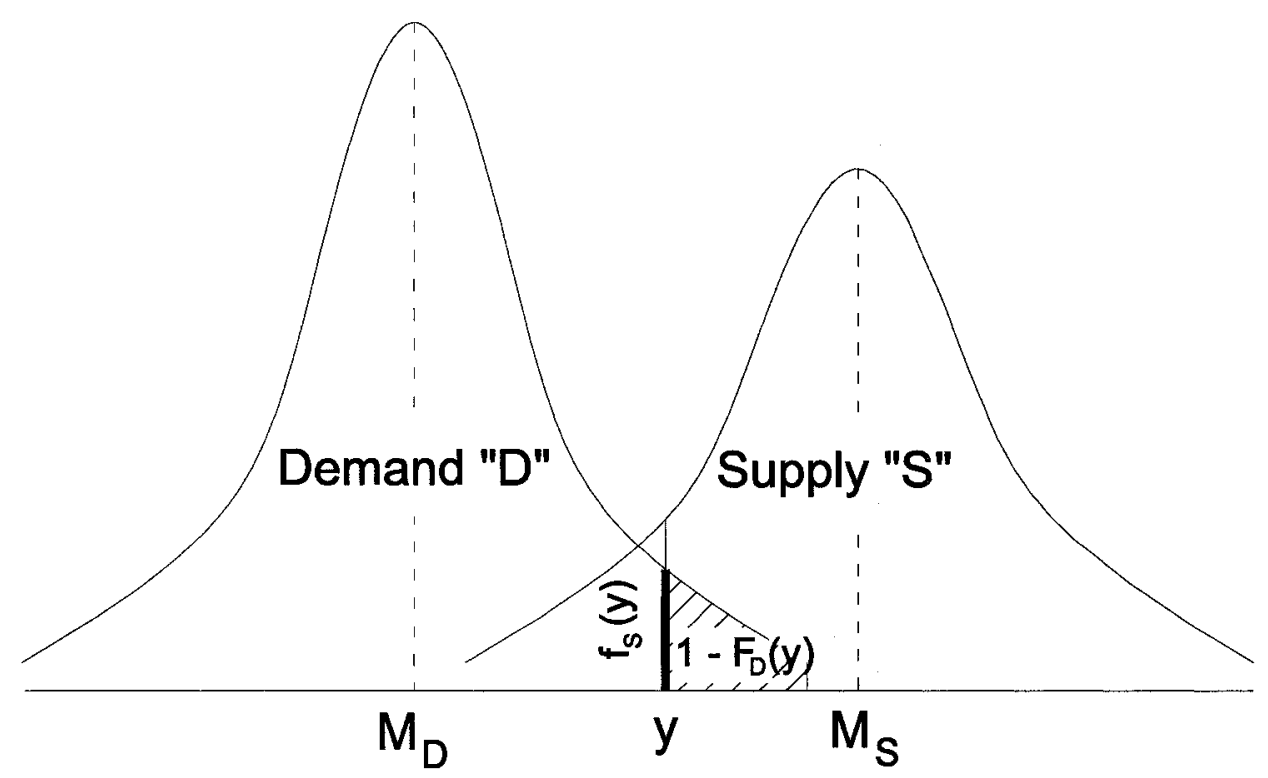

Figure 3-1: Prediction of $\mathrm{POH}$ in terms of $S$ and $D$ Distributions.

It should be mentioned, however, that the method requires full identification of the density functions for both $S$ and $D$. The integration in such form could also make the method even more complicated depending on the distribution type of both distributions. Furthermore, it could happen that either or both $S$ or $D$ does not follow known distribution types. Alternatively, a more applicable approach can be followed using one performance function that describes a failure event. The most widely used functions, as reported in the literature, are the factor of safety $(F)$ and safety margin $(M)$. From one side, $F$ is the ratio between $S$ and $D$.

$$
F=\frac{\operatorname{Supply}(S)}{\operatorname{Demand}(D)}
$$

Over decades, the use of $F$ has gained a great popularity in civil engineering applications especially in structure and geotechnical design branches. Given that both $S$ 
and $D$ are random variables, the value $F$ itself is random. A failure is then expected to take place when such ratio is less than one. Using this method, a system is logically safer by increasing the factor of safety. In addition, the ratio between the mean $S$ and mean $D$ is referred to as central factor of safety $(C F)$. Although this measure is simple, it does not include the variation of both $S$ and $D$ distributions since it deals only with mean values.

Furthermore, the $\mathrm{POH}$ can be expressed by evaluating the area under the distribution of $F$ where the ratio is less than one (see Figure 3-2-a). Similarly, $M$ can be described by the difference between $S$ and $D$ (Equation 3-3). Whenever a negative sign results from this equation, one should expect failure or hazard. In addition, the $M$-based $\mathrm{POH}$ is the area under the curve where the difference is less than zero (see Figure 3-2-b).

$$
M=\operatorname{Supply}(S)-\operatorname{Demand}(D)
$$

Equation 3-3

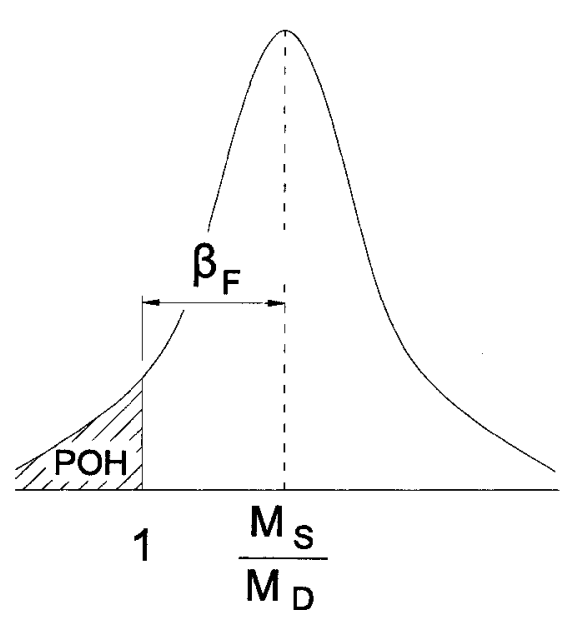

a) Factor of Safety

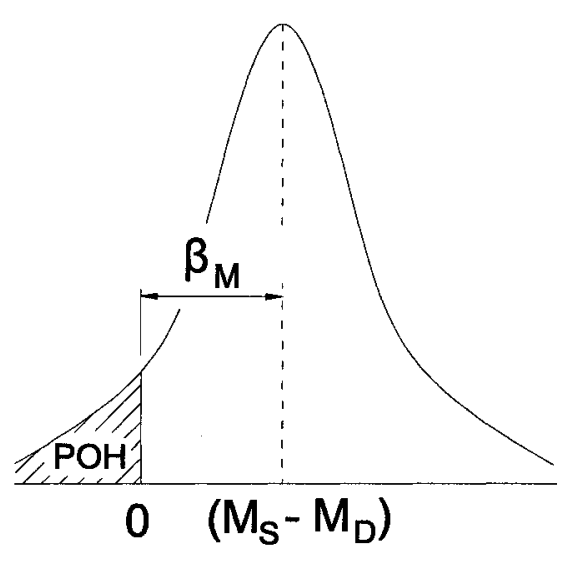

b) Safety Margin

Figure 3-2: Prediction of $\mathrm{POH}$ in terms of a Performance Function. 
Besides using $\mathrm{POH}$ as a measure of reliability, the use of the reliability index $(\beta)$ is not less important. Assuming a normal distribution for a performance function (i.e., $F$ or $M$ ), the corresponding $\beta$ can be calculated using either Equation 3-4 or Equation 3-5. This index represents how far the critical limit is from the mean of the performance function in units of standard deviation. It could be stated that as the value of $\beta$ increases the system becomes safer (Navin and Zheng 1998).

$$
\begin{aligned}
& \beta_{M}=\frac{E(M)}{\sqrt{\operatorname{var}(M)}} \\
& \beta_{F}=\frac{E(F)-1}{\sqrt{\operatorname{var}(F)}}
\end{aligned}
$$

\subsubsection{Types of Reliability Analysis}

For a performance function that is described in terms of other random variables, there are several probabilistic methods to obtain the distribution characteristics and moments (i.e., mean $(E(F))$ and variance $(\operatorname{var}(F))$ ) of such a function. These methods can be categorized into three main categories; namely (1) simulation, (2) first-order second-moment, and (3) point estimate. The first method, as the case with Monte Carlo simulation, requires computer capabilities to simply generate series of numbers (in rows) for each random variable (in columns). As shown in Figure 3-3, each column should follow a predetermined distribution with specific characteristics. A physical relationship then uses the set of numbers generated in each row to calculate the intended function. The 
calculated series of numbers would finally describe the probable distribution of the expected output.

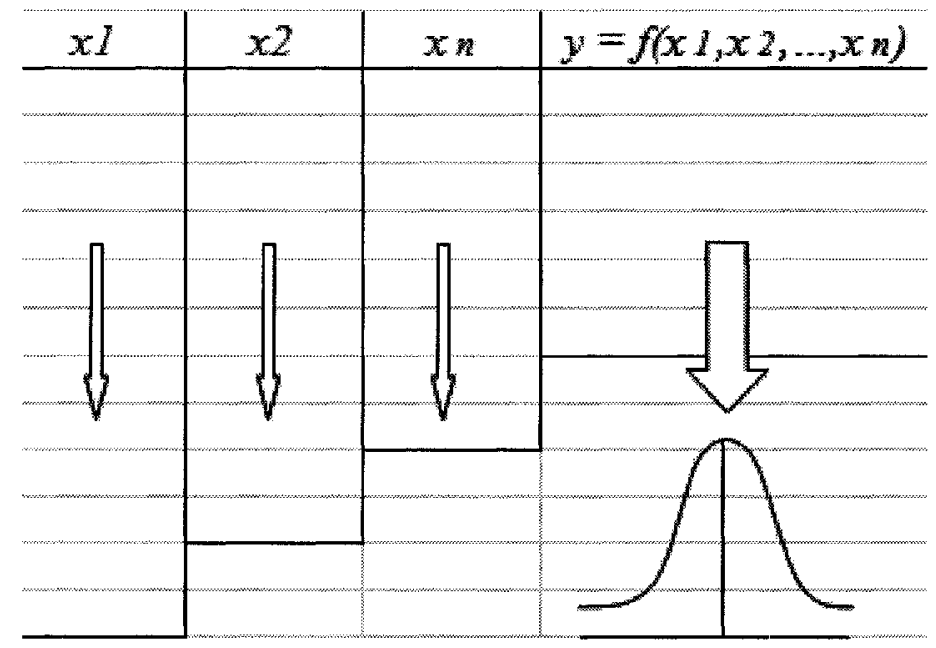

Figure 3-3: Simplification of Monte Carlo Simulation Technique.

The second method is based on the expansion of Taylor series about a point $\left(X=\bar{X}_{i}\right)$, as shown in Equation 3-6. Using only the first-order reliability method (FORM), two equations can describe the mean and variance of that equation, as shown in Equation 3-7 and Equation 3-8, respectively. The covariance term $\left(\operatorname{cov}\left[x_{1}, x_{2}\right]\right)$ in Equation 3-8 would account for the correlation between each pair of variables. This covariance can simply be calculated using the standard deviations of the two variables along with the correlation coefficient between these two variables as shown in Equation 3-9. It should be noted that the correlation coefficient ranges between -1 and +1 , for inversely and directly proportional relationships, respectively. The main disadvantage of this reliability method is the difficulty of conducting the derivations of complex equations. 


$$
\begin{aligned}
& F\left(x_{1}, x_{2}, \ldots, x_{n}\right) \\
& =\sum_{i=1}^{n} F\left(\bar{x}_{i}\right)+\frac{1}{1 !} \sum_{i=1}^{n}\left(x_{i}-\bar{x}_{i}\right) \frac{\partial F}{\partial x_{i}} \\
& +\frac{1}{2 !} \sum_{i=1}^{n} \sum_{j=1}^{n}\left(X_{i}-\bar{x}_{i}\right)\left(x_{j}+\bar{x}_{j}\right) \frac{\partial^{2} F}{\partial x_{i} \partial x_{j}} \\
& +\frac{1}{3 !} \sum_{i=1}^{n} \sum_{j=1}^{n} \sum_{k=1}^{n}\left(x_{i}+\bar{x}_{i}\right)\left(x_{j}+\bar{x}_{j}\right)\left(x_{k}+\bar{x}_{k}\right) \frac{\partial^{3} F}{\partial x_{i} \partial x_{j} \partial x_{k}}+\cdots \\
& E(Y)=F\left(\bar{x}_{1}, \bar{x}_{2}, \ldots, \bar{x}_{n}\right) \\
& \operatorname{var}(Y)=\sum_{i=1}^{n}\left(\frac{\partial F}{\partial x_{i}}\right)^{2}{\sigma_{x_{i}}}^{2}+\sum \sum_{i \neq j}^{n} \frac{\partial F}{\partial x_{i}} \frac{\partial F}{\partial x_{j}} \operatorname{cov}\left[x_{i}, x_{j}\right] \\
& \operatorname{cov}\left[x_{i}, x_{j}\right]=\rho_{x_{i}, x_{j}} * \sigma_{x_{i}} * \sigma_{x_{j}}
\end{aligned}
$$

Equation 3-7

where; $\bar{x}_{i}$ and $\sigma_{x_{i}}=$ mean and standard deviation of $x_{i}$; and $\rho_{x_{i}, x_{j}}$ and $\operatorname{cov}\left[x_{i}, x_{j}\right]=$ the correlation coefficient and covariance between $x_{i}$ and $x_{j}$.

The second order reliability method (SORM) could also be used by including second-derivative terms in Equation 3-6. This would make the calculation of the mean even more difficult; especially with complex equations (see Equation 3-10). However, the variance of SORM is usually taken the same as the one used in FORM for its simplicity. The third and less frequently used method in transportation engineering is the point estimate. Since the target of this research is not to recommend one technique against another, this last method will not be presented.

$$
E(Y)=F\left(\bar{x}_{1}, \bar{x}_{2}, \ldots, \bar{x}_{n}\right)+\frac{1}{2} \sum_{i=1}^{n} \sum_{j=1}^{n} \frac{\partial^{2} F}{\partial x_{i} \partial x_{j}} \operatorname{cov}\left[x_{i}, x_{j}\right]
$$




\subsubsection{Reliability Analysis in Geometric Design}

Unlike all other branches of design, geometric design has a distinctive property of the relationship between $S$ and $D$. Reliability analysis in many instances assumes that the $S$ and $D$ are functioning independently. For example, loads on a column in a specific structure may vary with the uncertainty of the dead load and the variation of the live load. On the other side, the column's capacity might independently vary in terms of the uncertainty in the strength of individual materials used to construct the column. Similarly in civil engineering, axle repetitions and loads on a specific road would fluctuate with time whereas the supply is uncertain because of the uncertainty of the material strength of the pavement.

In geometric design, the mechanism is different such that $S$ would influence $D$ through the interaction between the driver and the road alignment and other road features. The drivers usually adapt their speed, in part, on the perception of the road alignment. As mentioned by Fambro at al. (2000a), drivers usually select suitable operating speeds and other driving actions based on prevailing driving conditions. This fact causes $S$ to be linked to $D$ and hence creates a big challenge that is not normally seen in other engineering branches. For further illustration, let us assume a curve radius that increased from $R_{1}$ to $R_{2}$, as shown in Figure 3-4. Subsequently, operating speeds on that curve are expected to increase resulting in a new distribution of higher demanded sight distance. A comparison between the situations before and after changing the radius would lead to two different values of POH $\left(\alpha_{1}\right.$ and $\left.\alpha_{2}\right)$, each represents the percentage of drivers who accepted higher risk levels. One can assume that this percentage would increase on 
sections that are lacking consistency or designed at or near minimum design values, which might subsequently affect safety over such sections. For evaluation purposes, less challenge would be faced as operating speeds can be measured directly. However, for design and highway-realigning purposes, prediction of operating speeds would be the only way to conduct reliability analysis.

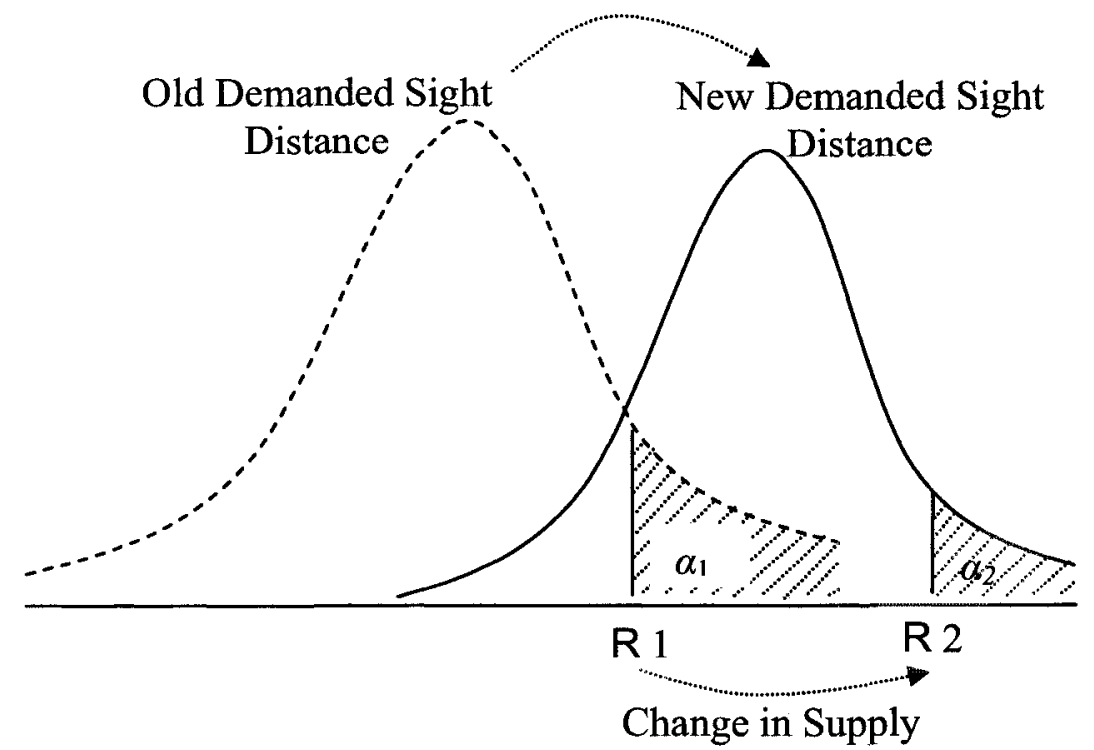

Figure 3-4: Dependency between $S$ and $D$ in Geometric Design.

\subsection{Geometric Design Framework}

After the recognition of the role that the reliability analysis can play in geometric design, a framework was suggested to provide a simple and effective geometric design process. The framework shown in Figure 3-5 is based on the probabilistic approach and reliability analyses that would consider common fluctuations among drivers' behaviours and other contributing factors. 


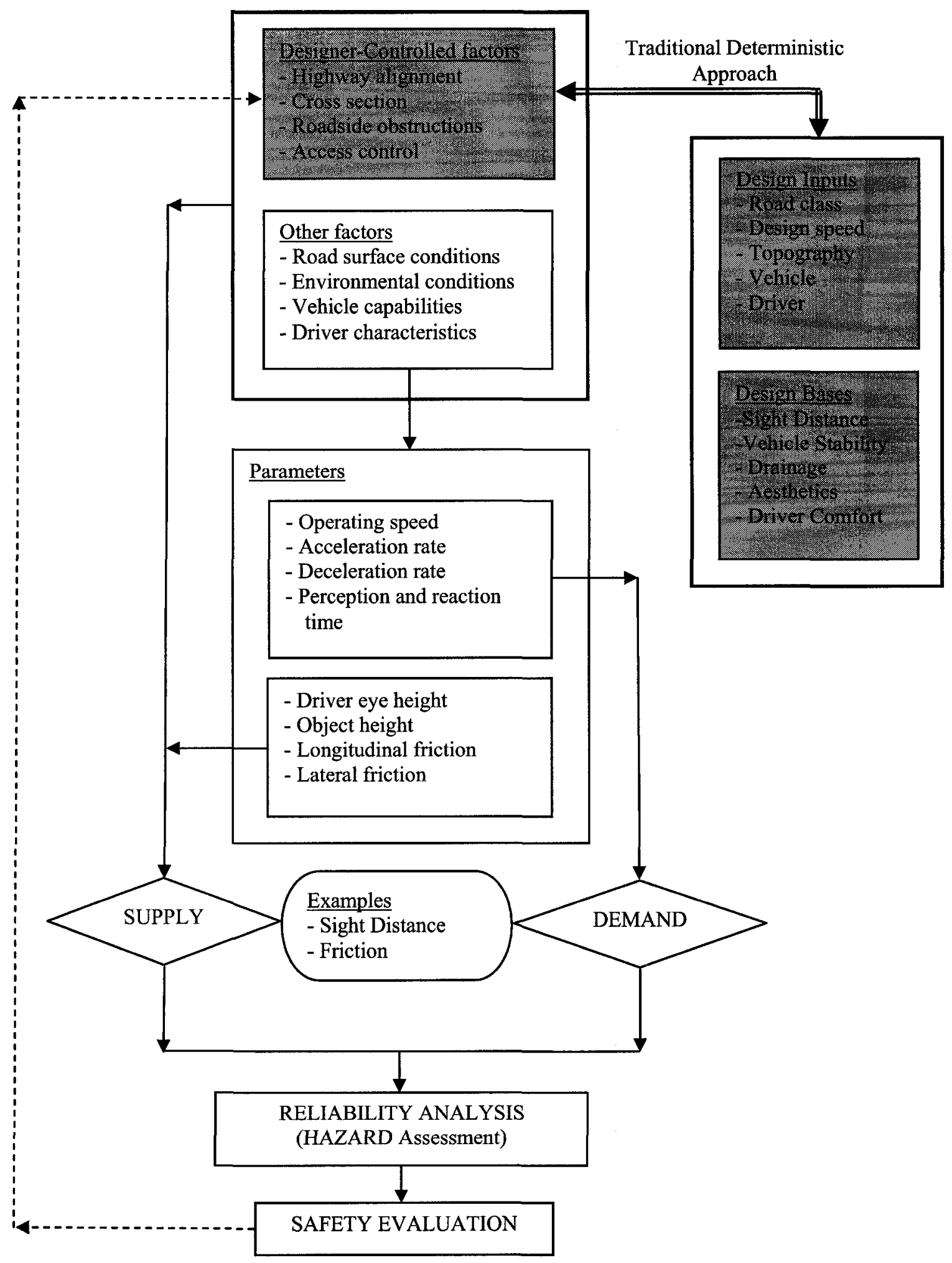

Figure 3-5: Reliability-Based Framework for Highway Geometric Design. 
The shaded part in this diagram illustrates the traditional deterministic approach used in current design guides as suggested by (Hassan 1996). The framework starts by surveying all design inputs such as road class, speed, topography, vehicle characteristics, and driver behaviour. With all this information in hand, a deterministic approach could be applied to estimate an initial design for a specific highway feature. This design could be conducted using several design bases including sight distance, vehicle stability, drainage, aesthetics, and driver comfort (Hassan et al. 1998). As mentioned earlier, these bases can be identified using either $2 \mathrm{D}$ or $3 \mathrm{D}$ design approaches. The main difference is the way a highway is analyzed in order to determine the physical relationships that describe the design parameters. The 2D design approach, as seen in most of current design guides, uses either horizontal or vertical highway projections whereas the 3D approach considers the combination of horizontal and vertical alignments.

Using these bases, both horizontal and vertical highway alignments along with other elements related to cross-section, roadside, and access controls are currently designed following the traditional deterministic design concept. This concept determines minimum design criteria for what one may call designer-controlled highway elements. Examples are radii of horizontal curves and lengths of vertical curves. The initial design given by the deterministic approach in addition to other factors such as road surface conditions, environmental conditions, vehicle capabilities, and driver characteristics may somehow cause variations of different parameters and driving actions such as operating speeds, acceleration, and deceleration rates. A good design is believed to be the one that aims at reducing such variation since collisions are expected to increase with improper speed adaption (Al-Masaeid et al. 1994). While these parameters can be predicted and 
somehow controlled, others still vary randomly and independently from the highway design such as perception and reaction time, driver eye and object heights, and friction coefficients.

The variability of the design parameters would normally describe the variation of both $S$ and $D$. Factors such as speed, acceleration and deceleration rate, and perception and reaction (PR) time would normally cause variation in $D$ whereas variations in $S$ would be influenced by factors such as the driver eye height, object height, and friction coefficient along with the road characteristics. Using physical relationships and appropriate distributions and correlations between all these variables, a range of $D$ might be mathematically derived using probability/reliability analysis. This range can be checked against the corresponding range of $S$ using either the $2 \mathrm{D}$ or $3 \mathrm{D}$ design approaches.

The comparison between $S$ and $D$ would finally yield a certain hazard expected from a specific design. This hazard reflects how far $S$ meets $D$ and hence a risk can be quantified. This can be applied on several design considerations such as sight distance and friction coefficient. Moreover, the safety performance can be evaluated by capturing the relationship between the hazard degree and actual collision data. The criteria of a highway features can then be changed repeatedly until the desired safety performance is finally reached. Furthermore, It would be beneficial for decision makers to compare between different alternatives with the possibility to alter a certain deign to meet a predetermined risk level. 


\subsection{Research Contribution}

In light of the proposed framework, and because of time and effort limitations, the expected contribution of this research is outlined in this section. The suggested methodology described earlier will be applied to the sight distance design basis. The current deterministic design approach will be first used as the best initial estimate of the design criteria. Then, such design will be assessed as described in Section 3.2. As needed, the design may then be altered and re-evaluated using the same procedures. As shown in Figure 3-6, the stopping sight distance (SSD) was selected to represent the required sight distance (RqSD) as a demand whereas the 2D and 3D available sight distances (AvSD) will be considered as the supply. Using different combinations of these design elements, hazard can be assessed.

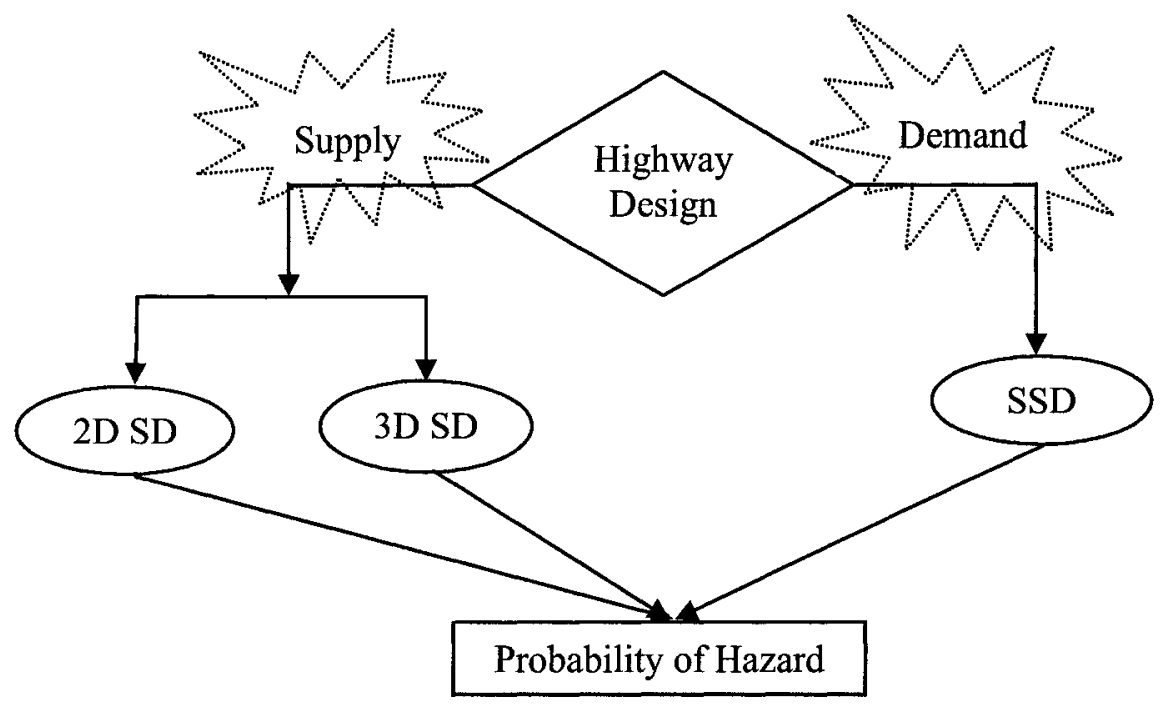

Figure 3-6: The Outline of the Expected Contribution of this Research. 
It should be mentioned, however, that the use of $\mathrm{POH}$ in highway geometric design can be described as the most realistic hazard measure compared to other measures presented earlier in Section 3.1.2. The rationale of this selection is that no hazard can be linked to insufficient sight distance if all drivers traversing a specific road section are expected to have greater AvSD than RqSD. This would happen if both AvSD and RqSD distributions are far from each other such that $\mathrm{POH}$ is zero. On the other side, the use of reliability indexes may still indicate different risk levels even though the two distributions are not intersecting. Since this fact contradicts with the argument drawn here, the use of both reliability indexes based on $F$ or $M$ could be inappropriate. In addition, the $\mathrm{POH}$ reflects the percentage of drivers who may suffer insufficient sight distance while the reliability indexes are lacking a useful meaning to highway designers. For these reasons, POH was considered in this research as a suitable hazard measure.

\subsection{Sight Distance Evaluation System "SDES"}

In this section, a tool, namely sight distance evaluation system (SDES), was developed and programmed to meet the objectives of the study and to serve the contribution of the research. The program was prepared using Visual Basic.Net objectoriented programming language taking advantage of its great graphical capability, monitoring flexibility, and user friendly interface. As mentioned earlier, the probabilistic approach that forms the core of the proposed work in this research can be conducted using several methods including the analytical technique and the simulation method. With the fact that the $3 \mathrm{D}$ AvSD is part of the expected outcome, the simulation method may seem to be the only applicable approach. The reason for this is the inexistence of 
closed-form solutions of 3D AvSD required to conduct the analytical reliability technique.

In general, SDES is an efficient tool that applies the basic reliability principles to test how far AvSD satisfies RqSD at a given road section. Using SDES, this can be presented using three profiles representing AvSD, RqSD, and the probability that a driver may require more sight distance than needed; namely probability of hazard (POH). The $\mathrm{POH}$ profile is helpful to locate road sections with relatively higher risk than normal, and hence countermeasures can be taken to mitigate expected consequences on such sections. As shown in Figure 3-7, the calculation framework is divided into two main stages (1) pre-analysis stage and (2) sight distance assessment stage. It should be mentioned that the shaded part in this graph represents the work done in mid 1990s by Hassan (1996) to calculate 2D and 3D AvSD. More discussion in this regard will be presented in Section 3.4.2.c and later in CHAPTER 5.

\subsubsection{SDES Framework-Pre-Analysis}

The first stage of the framework is preparatory in which the alignment and obstructions are generated giving the chance for users to customize the road section under study. The user can also specify several settings and options that narrow the design base and target. This stage consists of three main steps including alignment and obstruction generation, design parameters identification, and options for design flexibility. 


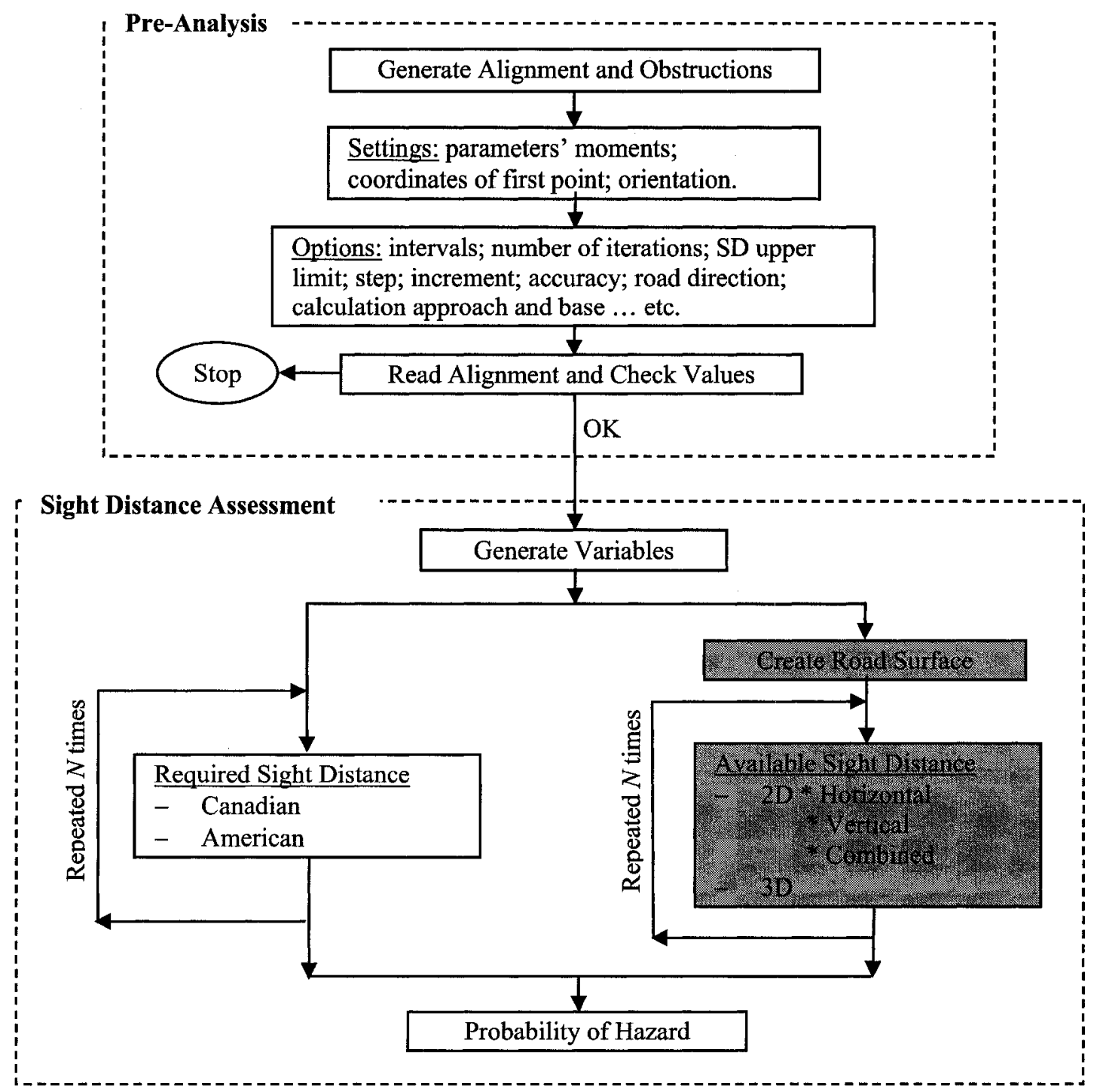

Figure 3-7: Suggested Framework to Evaluate Sight Distance Sufficiency.

\subsection{1.a Generate alignment and obstructions}

As shown in Figure 3-8-A, an alignment is identified by its horizontal alignment, vertical alignment, cross-sections, points of each spline-grade segment, and horizontal and vertical obstructions. 


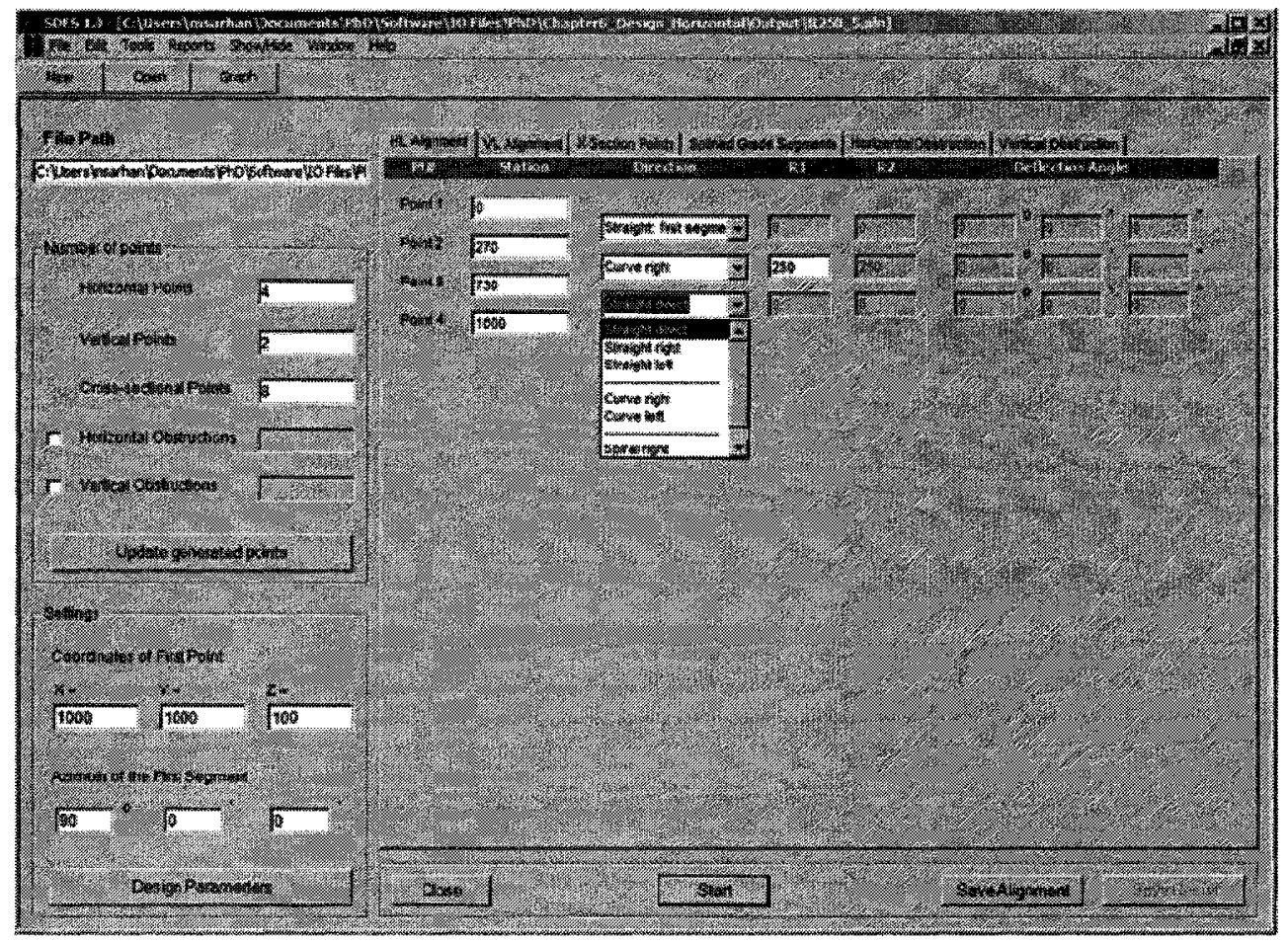

A) SDES Main Window for Alignment Configurations.

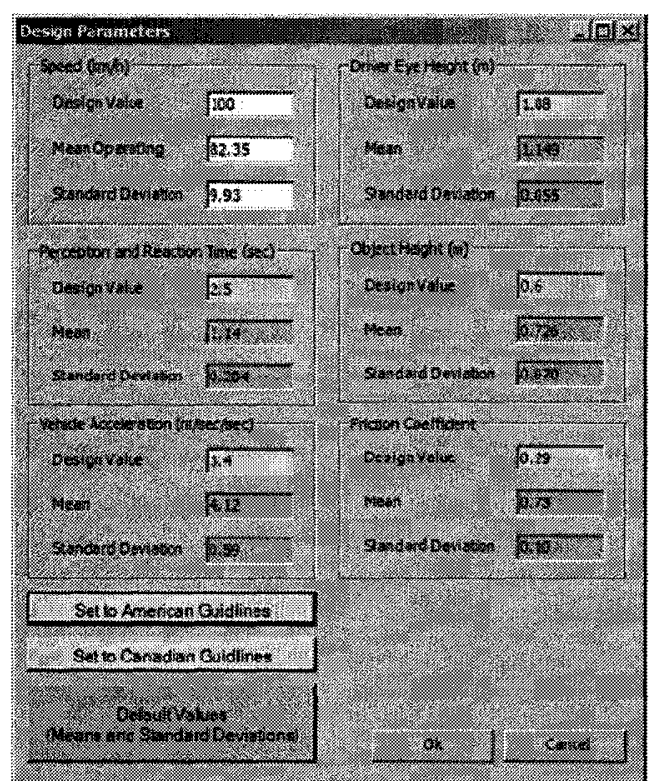

B) Settings of different Parameters.

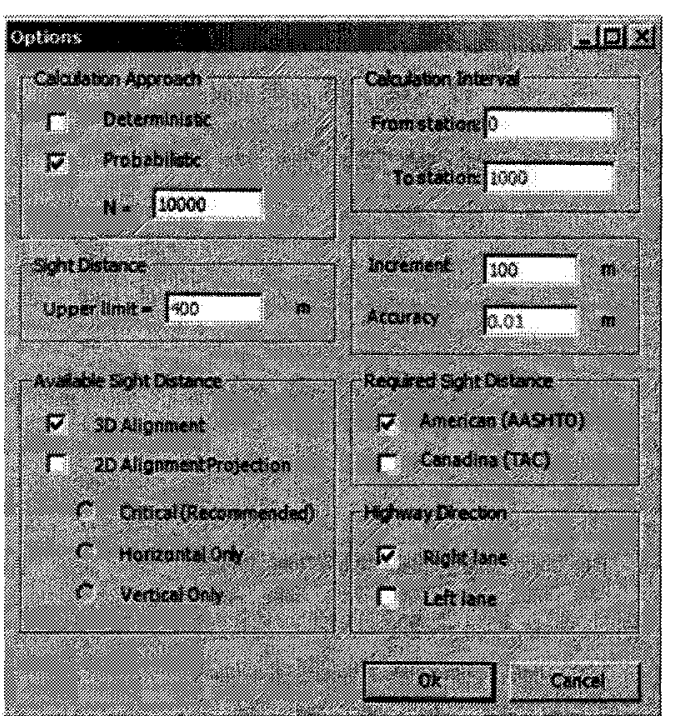

C) Options for Design Flexibility.

Figure 3-8: SDES Main Interfaces. 
For the number of horizontal points, it includes the first and last points of the alignment in addition to every point that separates two different elements of a straight segment, horizontal curve, or transition curve. Each two successive points bound a road segment that requires a direction, bounding radii, and a deflection angle to identify such segment. As for the number of vertical points, it includes the first and last points along with the points separating two different elements of a tangent, crest or sag vertical curve, or spline-grade segment. Each vertical element is defined using the element type and bounding grades. For a spline-grade segment, a box is also provided for users to set the number of points defining such segment.

Moreover, each cross-section is generated at every change in one or more of its elements such as lane or shoulder width, side slope, or superelevation rate. Another interface is also available to enter a station and elevation for every point of those forming spline-grade segments. Furthermore, the tool provides an opportunity to set horizontal obstructions that may consist of one, two, or three edge points. Vertical obstructions such as overpasses may also be identified as perpendicular or skewed and each requires stations, elevations, and widths of its main points.

\subsection{1.b Design parameters identification and other settings}

As shown in Figure 3-8-B, the user is required to specify the design value, mean, and standard deviation of each of six variables including operating speed, PR time, deceleration rate, friction coefficient, driver eye height, and object height. The first four out of these six variables are used to calculate the RqSD based on both the Canadian and American guidelines while the last two are required for 2D and 3D AvSD calculations. In 
addition, the design values are used to conduct the deterministic analysis whereas the mean and standard deviation are used to generate parameters' distributions for the probabilistic analysis. These distributions should express the variation characteristics of such design parameters as the simulation reliability technique would normally require. In addition, SDES can read pre-generated numbers from other sources as a second alternative of describing the design parameters. This may be useful to read numbers that were created using more efficient tools such as Matlab. It should be noted that only uncorrelated uniform distributions can be generated within the Visual Basic.Net platform. In addition to parameters identification, the user specifies the Cartesian coordinates of the first point of an alignment along with the azimuth of the first segment.

\subsection{1.c Options for design flexibility}

As shown in Figure 3-8-C, numerous options are available in SDES to describe the characteristics of a specific run. These options include the selection of an interval of the highway over which the sight distance will be evaluated. In addition, the number of iterations to be used in the simulation process can be identified. Increasing this number may increase the confidence of the output and enhance the outcome. The user can also set an upper limit of AvSD over which AvSD is considered fairly safe and hence will not be calculated. Moreover, the increment and accuracy should be considered carefully since it may influence considerably the calculation duration and precision.

There are other options that SDES offers for easy navigation through different design and evaluation approaches. For example, RqSD can be calculated based on either or both Canadian and American guidelines. The AvSD can also be evaluated in either 2D 
or $3 \mathrm{D}$ environment. Furthermore, either a deterministic or probabilistic approach can be adapted on one or two of the road directions. In the last step of the pre-analysis stage, SDES reads and checks all values, signs, and ranges of entered configurations to ensure logical data entry and to eliminate possible typos before moving to the second stage. If one or more values are detected for modification, a warning message is displayed.

\subsubsection{SDES Framework - Sight Distance Assessment}

In this second stage, a sight distance assessment is conducted following either a deterministic or probabilistic approach as specified in the first stage. In the deterministic approach, a single iteration is used to calculate two values of RqSD and AvSD utilizing near-worst design values for all design parameters as per the design guides. On the other hand, using the probabilistic approach, the calculation process is repeated for $N$ times utilizing a distribution for each design parameter and the hazard is then evaluated. In this latter scenario, a generating function may be required to produce random distributions. The following sections describe the assessment process in more detail.

\subsection{2.a Variables generator}

In Visual Basic.Net platform, it is possible to generate random numbers that range from zero to one. However, only uniform distributions can be generated with no capabilities to generate others such as normal or Gamma distributions. Throughout the literature, two methods were found to convert a uniform distribution to a standard normal distribution including, and not limited to, (1) basic form and (2) polar form. The first 
approach produces a normal distribution from two uniform distributions that range from 0 to 1 .

$$
Z=\sqrt{\left(-2 \log \left(u_{1}\right)\right)} * \cos \left(2 \pi * u_{2}\right)
$$

Equation 3-11

where; $Z=$ standard normal distribution (mean $=0$ and standard deviation $=1$ ); and $u_{1}$ and $u_{2}=$ two uniform distributions each has a range from 0 to 1 .

The second approach also uses two random variable, $v_{1}$ and $v_{2}$. However, the range of each variable is -1 to 1 . The two variables can also be converted to a standard normal distribution using the polar form as follows:

$$
\begin{aligned}
& Z=v_{1} \sqrt{(-2 \log R) / R} \\
& R=\sqrt{v_{1}^{2}+v_{2}^{2}} ; R<1
\end{aligned}
$$

where; $v_{1}$ and $v_{2}=$ two uniform distributions each has a range from -1 to 1 .

In cases where $R$ is greater than or equal to 1 , another pair of $v_{1}$ and $v_{2}$ is generated and a new value of $R$ is recalculated. The difference between the two approaches is that the second is faster because it is simpler to compute. However, little difference can be observed between the two methods considering the current computer capabilities. Using either method, it is possible to generate a normal distribution with certain mean and standard deviation.

$$
N=\mu+\sigma Z
$$

\section{Equation 3-14}

where; $N=$ normal distribution; $\mu=$ mean; $\sigma=$ standard deviation; and $z=$ standard normal distribution. 
It should be noted that, in cases where other distributions than normal are needed and also where variables have known correlation coefficients, there is a need for a more powerful tool to generate such variables. The generated variables can then be imported to SDES for further processing.

\subsection{2.b Required sight distance}

As mentioned earlier, the stopping sight distance was selected to represent RqSD as the minimum requirement that has to be achieved on all road types. The two main equations listed in both TAC (1999) and AASHTO (2004) were formulated based on the basic laws of physics as a simple summation of two distances.

$$
\begin{aligned}
& S S D=0.278 V t+\frac{V^{2}}{254(f \pm G)} \\
& S S D=0.278 V t+\frac{V^{2}}{254\left(\frac{a}{9.81} \pm G\right)}
\end{aligned}
$$

where; $t=$ perception and reaction time $(\mathrm{s}) ; V=$ initial speed $(\mathrm{km} / \mathrm{h}) ; f=$ coefficient of friction between tires and roadway; $a=$ deceleration rate $\left(\mathrm{m} / \mathrm{s}^{2}\right)$; and $G=$ percent grade divided by 100 .

The direct application of these two equations assumes that a road segment has a constant grade on a substantial length of the road. Because this is not always the case, a method was developed to simulate a stopping attempt considering variations in longitudinal grade. The grade at a specific location can be calculated in terms of the road elements and the surface created in an earlier step. The methodology is presented in more 
detail in Section 4.1. In SDES, the user may also select either AASHTO or TAC formula as a calculation base.

\subsection{2.c Road surface creation and available sight distance}

The AvSD estimation takes the advantage of two existing DOS-based programs written using the Quick Basic programming language (Hassan 1996; Hassan et al. 1996; Hassan et al. 1997). The two programs were developed based on the concept of finite element in $2 \mathrm{D}$ and $3 \mathrm{D}$ projections. The $2 \mathrm{D}$ program simulates the current analytical methods in current design guides whereas the $3 \mathrm{D}$ program creates a $3 \mathrm{D}$ mesh that fits the road surface and side slopes with 3-node, 4-node, and 6-node elements. These elements are then checked for intersection with the sight line that connects the driver eye to an object located at distance $S$ from the driver eye. If no intersection is observed, the distance $S$ is increased and rechecked until the intersection occurs. Within this framework, four different approaches can be adapted to calculate AvSD including 2Dhorizontal, 2D-vertical, 2D-combined, and 3D. This can be beneficial to compare between 2D and 3D design approaches. Further discussion will be presented in CHAPTER 5.

\subsection{2.d Hazard evaluation}

In the probabilistic approach, the methods presented in Section 3.4.2.b and 3.4.2.c are repeated for $N$ times using pre-generated distributions of different parameters to yield two other distributions for AvSD and RqSD. These two latter distributions are used to evaluate the hazard using the measures presented earlier in Section 3.1.2. Such measures 
are calculated based on comparisons between individual values of both AvSD and RqSD distributions. In this regard, the distribution of the factor of safety $(F)$ is first formed by dividing each two corresponding values in both AvSD and RqSD distributions.

$$
F_{i}=\frac{A v S D_{i}}{R q S D_{i}} \quad ;(i=1 \text { to } N)
$$

In addition, a code was added to SDES to filter those cases where such ratio is less than one. The $\mathrm{POH}$ is then quantified as the percentage of those cases to all others (e.g., $N$ number of repetitions). The value of $\beta_{\mathrm{F}}$ (based on $F$ ) is also calculated as the distance, measured in units of standard deviation, from the mean to the state limit (ratio = 1), assuming a normal distribution for $F$.

$$
\beta_{F}=\frac{\left(\mu_{F}-1\right)}{\sigma_{F}}
$$

Equation 3-18

Similarly, another distribution of the safety margin $(M)$ is formed by subtracting each two corresponding values in AvSD and RqSD distributions. It should be mentioned that the same value of $\mathrm{POH}$ is obtained by counting those cases where such difference is less than zero. However, the $\beta_{\mathrm{M}}$, as shown in Equation 3-20, is not necessarily to be equal to $\beta_{\mathrm{F}}$ since it depends on the distribution type.

$$
\begin{aligned}
& M_{i}=A v S D_{i}-R q S D_{i} ;(i=1 \text { to } N) \\
& \beta_{M}=\frac{\mu_{M}}{\sigma_{M}}
\end{aligned}
$$

As mentioned before, only $\mathrm{POH}$ measure will be used in this research even though SDES calculates all hazard measures described above. 


\subsection{Summary}

In this chapter, a brief discussion was presented covering reliability analysis from different prospective including basic concept, measures of reliability, and types of analysis. Difference between application of reliability in geometric design and other design branches was also highlighted. In addition, a geometric design framework was suggested. Considering normal time limitation, the design basis of sight distance was selected as an application example of the suggested framework. Hazard evaluation procedures were demonstrated and a tool, namely SDES, was developed to apply such reliability-based design concept to compare the sight distance required by drivers (RqSD) and that actually available to them (AvSD). 


\section{CHAPTER 4}

\section{Probabilistic Required Sight Distance}

In this chapter, the required sight distance (RqSD) is assessed using both the deterministic and probabilistic approaches. In Section 4.1, few approximations in the current design practice are highlighted. Section 4.2 presents a new algorithm to calculate a precise RqSD that considers the continuous change in grade and friction during a stopping scenario. In addition, the RqSD is evaluated using both deterministic and probabilistic design approaches in Section 4.3. Moreover, Section 4.4 displays a pioneered approach that considers both American and Canadian RqSD formulas at the mean time. Furthermore, friction data were collected at different weather and road surface conditions and findings are outlined in Section 4.5.

\subsection{Approximations in Current Practice}

There are different types of required sight distances that are all required to operate safely at different driving situations. Among those types is the required stopping sight distance (RqSD). By definition, RqSD is the distance a driver would need to see ahead to stop in cases that urge stopping for safe operations and manoeuvres. As mentioned before, two equations were stated in North American guides to estimate stopping sight distances (SSDs). The two formulas describe the SSD in terms of the vehicle speed, perception and reaction time, deceleration rate, and longitudinal friction. 
Formula in TAC (1999):

$S S D=0.278 V t+\frac{V^{2}}{254(f \pm G)}$

Equation 4-1

Formula in AASHTO (2004):

$S S D=0.278 V t+\frac{V^{2}}{254\left(\frac{a}{9.81} \pm G\right)}$

Equation 4-2

where; $S S D=$ stopping sight distance (m); $t=$ PR time (second); $V=$ initial speed $(\mathrm{km} / \mathrm{h})$; $f=$ coefficient of friction between tires and roadway; $a=$ deceleration rate $\left(\mathrm{m} / \mathrm{s}^{2}\right)$; and $G=$ percent grade divided by 100 .

The two SSD formulas are based on basic laws of physics that express the exact distance needed by a driver to stop. However, the estimates of the parameters in these formulas are usually simplified for design purposes. This could result in SSD values that may considerably underestimate or overestimate drivers' need. In the following few sections, two aspects will be stressed including (1) the longitudinal grade and (2) longitudinal coefficient of friction. A more accurate algorithm will also be presented to determine a precise SSD profile along road segments by simulating actual stopping scenarios at predetermined intervals.

\subsubsection{Consideration of Longitudinal Grade}

Since road gradient has a direct influence on vehicles' speed, the vertical alignment must be considered in the SSD calculations. In both TAC and AASHTO formulas, the parameter $G$ should account for the longitudinal grade. However, there are several approaches one can possibly follow to correct SSD values with regard to longitudinal grade. Generally, a positive value is used for road segments with a 
substantial constant upgrade whereas a negative value would describe constant downgrades. However, it is often hard to judge on the appropriate value/sign of the grade when a road segment has successive sharp grades and single or consecutive vertical curves. For simplicity in current design guides, a zero grade is assumed for the design of vertical curves according to the sight distance design basis. This was returned to the assumption that "sight distance available on downgrades is larger than on upgrades, more or less automatically providing the appropriate corrections for grade" (AASHTO 2004). This assumption may underestimate the drivers' need and therefore provide a certain hazard degree on drivers' performance.

A more representative way to include the grade in the design is the use of an average grade of certain road length over which a vehicle is attempting to stop. For example, a SSD value over a crest curve may use the average grade of both curve approaches as an acceptable estimate to be substituted in the SSD formulas. This averagegrade approach would actually underestimate the SSD value near the downgrade part, where drivers may urgently need to stop in order to avoid hitting a hidden object due to the vertical road curvature. The average-grade approach may also give an approximate SSD value that does not correspond to a specific section but rather represents a rough design criterion for the entire segment. Moreover, the approach is not applicable in case of irregular spline-grade sections that connect a number of points each defined by its station and elevation. For these reasons, the efficiency of current practice to account for the longitudinal grade can be considered inappropriate and therefore another approach was developed, as shown in Section 4.2. 


\subsubsection{Influence of horizontal Curvature on Longitudinal Friction}

By definition, the friction is the force that is created when two surfaces are sliding over each other. In general, when a brake is applied, the friction between the vehicle tyres and the pavement surface forms a retarding force that helps vehicles to stop. As shown in Figure 4-1, this friction force is the product of the normal force $(N)$ on each tyre and the coefficient of friction $(F)$ between the tyre and the pavement. On curved segments, this friction force would have two main components in response to the centripetal forces due to horizontal curvature. The first component is the longitudinal friction coefficient, $f_{1}$, which causes reduction in the operating speed when brakes are applied whereas the second component, $f_{\mathrm{s}}$, is the side friction coefficient that would provide an adequate lateral balance of operating vehicles. Using the equilibrium between all forces acting on the vehicle in the lateral direction, the value of $f_{\mathrm{s}}$ can be simply estimated using Equation 4-3. Equation 4-4 can then be used to predict the instantaneous value of $f_{1}$ at a certain road section. On straight segments, the value of $f_{1}$ is equal to $F$ while the difference increases with increasing the road's horizontal curvature.

$$
\begin{aligned}
& f_{s}=\frac{V^{2}}{127 R}-e \\
& f_{l}=\sqrt{F^{2}-f_{s}^{2}}
\end{aligned}
$$

Equation 4-3

Equation 4-4

where; $F=$ friction coefficient; $f_{1}=$ longitudinal friction component; $f_{\mathrm{s}}=$ lateral friction component; $V=\operatorname{speed}(\mathrm{km} / \mathrm{h}) ; R=$ curve radius $(\mathrm{m}) ;$ and $e=$ superelevation rate. 

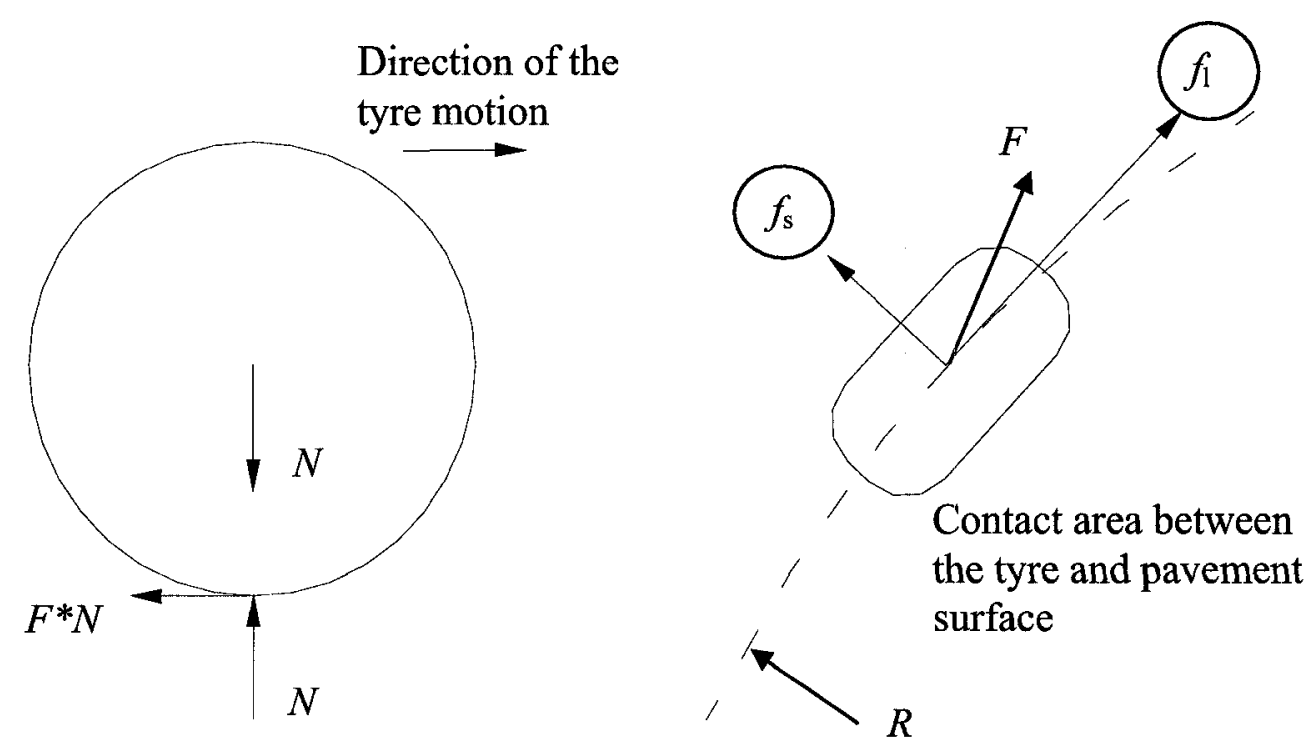

Figure 4-1: Forces Acting on a Vehicle's Tire at Stopping on Horizontal Curves.

It should be mentioned that the reduction of friction on horizontal curves was discussed in TAC (1999). It was mentioned that "on tight curves, stopping sight distances may need to be increased to compensate for increased braking distances caused by lateral friction effects." As shown in Table 4-1, the increase of SSD on curves varies from 0.3 to $8.8 \%$ compared to SSD on straight segments, depending on the design speed and the superelevation rate. Based on the suggested adjustments of SSD, it was stated in TAC (1999) that the calculation of minimum curve radii should consider the corrected SSD values listed in the table and the use of the equations is even more demanding if more precision is required. 
Table 4-1: RqSD on Horizontal Curves Considering Reduced Friction (Adapted from TAC 1999).

\begin{tabular}{ccccc}
\cline { 3 - 4 } & & & \multicolumn{2}{c}{ SSD on Curve $(\mathrm{m})$} \\
\hline $\begin{array}{c}\text { Design Speed } \\
(\mathrm{km} / \mathrm{h})\end{array}$ & $\begin{array}{c}\text { Operating } \\
\text { Speed }(\mathrm{km} / \mathrm{h})\end{array}$ & $\begin{array}{c}\text { Normal SSD } \\
(\mathrm{m})\end{array}$ & $\mathrm{e}=0.06$ & $\mathrm{e}=0.08$ \\
\hline 40 & 40 & 45 & 46 & 46 \\
50 & $47-50$ & $60-65$ & $60-66$ & $60-66$ \\
60 & $55-60$ & $75-85$ & $77-90$ & $77-90$ \\
70 & $63-70$ & $95-110$ & $97-120$ & $97-120$ \\
80 & $70-80$ & $115-140$ & $116-152$ & $116-151$ \\
90 & $77-90$ & $130-170$ & $134-180$ & $134-181$ \\
100 & $85-100$ & $160-210$ & $160-218$ & $160-219$ \\
110 & $91-110$ & $180-250$ & $181-259$ & $181-259$ \\
120 & $98-120$ & $200-290$ & $205-302$ & $204-301$ \\
130 & $105-130$ & $230-330$ & $229-338$ & $229-338$ \\
\hline
\end{tabular}

\subsection{New Algorithm for Exact RqSD}

The suggested method replaces the conventional use of single values for $G$ and $f$ that is usually used for its simplicity. Considering the current computer and programming efficiency, the new methodology utilizes the instantaneous grade $\left(G_{\mathrm{i}}\right)$ and longitudinal friction $\left(f_{\mathrm{i}}\right)$ to calculate an exact $\mathrm{RqSD}$.

\subsubsection{Calculation Methodology}

Figure 4-2 shows a schematic of the suggested methodology in which a vehicle is attempting to stop over an assumed road stretch. The vertical alignment consists of a simple vertical curve that connects two grades $\left(G_{1}\right.$ and $\left.G_{2}\right)$ whereas the vehicle passes through a tangent, spiral, and horizontal curve with a radius $R$. The suggested approach depends on dividing the road stretch into small intervals (e.g., $1 \mathrm{~m}$ ) with each segment has two values representing average grade and friction. Following the perception-reaction distance (i.e., $0.278 V t$ ), the speed at the end of each small segment is calculated as: 


$$
V_{i+1}=\sqrt{V_{i}^{2}-254\left(f_{i} \pm G_{i}\right)}
$$

Equation 4-5

where; $V_{\mathrm{i}}$ and $V_{\mathrm{i}+1}=$ speed at the start and end of a small segment $i$, respectively; $f_{\mathrm{i}}=$ reduced friction coefficient due to horizontal curvature; and $G_{\mathrm{i}}=$ instantaneous percent grade.

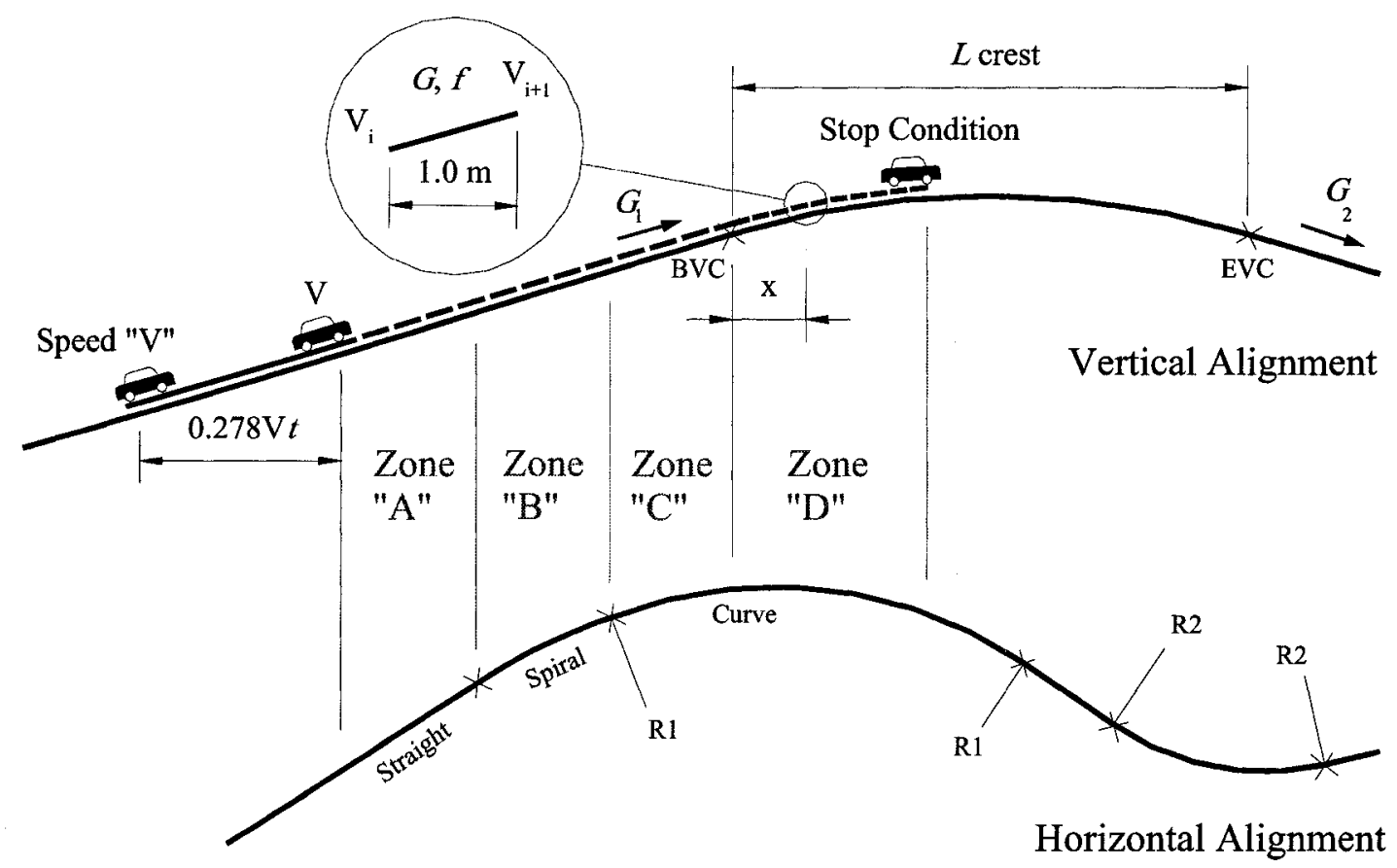

\begin{tabular}{|l|l|l|l|l|}
\hline Zone & "A" & "B" & "C" & "D" \\
\hline Grade & $G_{1}$ & $G_{1}$ & $G_{1}$ & $G_{1}+x / k$ \\
\hline Radius & Infinity & Variable & $R_{1}$ & $R_{1}$ \\
\hline Friction Coefficient & $f$ & $f_{1}$ & $f_{1}$ & $f_{1}$ \\
\hline
\end{tabular}

Where $G=$ percent grade of the first tangent; $x=$ distance from BVC; $k=$ rate of vertical curvature; $f=$ friction coefficient; $f 1=$ longitudinal component of the friction coefficient.

Figure 4-2: Suggested Algorithm to Calculate SSD.

If a speed of zero is reached at the end of one segment, a stop condition is assumed and the process is repeated within this small segment with finer intervals for 
more accurate estimation of SSD. Finally, the perception-reaction distance is added to the summation of all prior segments. For tangents (Zone $A$ through $C$ ), a constant grad is taken for all small intervals whereas Equation 4-6 is used to calculate the grade of each small interval on crest or sag vertical curves (Zone $D$ ).

$$
G_{i}=G_{1}+\frac{x}{k}
$$

Equation 4-6

where; $G_{1}=$ percent grade of the first curve tangent; $x=$ distance from the beginning of vertical curve; and $K=$ length of vertical curve per 1 percent change in grade.

As for the longitudinal friction values, the radius $\left(R_{\mathrm{i}}\right)$ at the mid-point of each small segment is first calculated. The $R_{\mathrm{i}}$ is simply the curve radius on horizontal curves (Zone $C$ and $D$ ) whereas, on transition curves (Zone $B$ ), this value can be calculated in terms of the spiral parameter and the distance from the beginning of the transition curve.

$$
R_{i}=R_{1} \pm \frac{A^{2}}{x_{i}}
$$

where; $R_{\mathrm{i}}=$ average radius of segment $i ; R_{1}=$ radius at the beginning of the transition curve; $A=$ spiral parameter; $x_{\mathrm{i}}=$ distance from the beginning of the transition curve.

Once the radius of each segment is calculated, the longitudinal friction can be obtained by substituting back in Equation 4-3 and Equation 4-4. The exact RqSD using such approach can also be applied to the AASHTO SSD formula with even less complications. This is because the deceleration rate is not normally affected by road curvature. Therefore, only the influence of longitudinal grade is taken into consideration. 


\subsubsection{TAC and AASHTO RqSD Profiles}

In order to test how far the longitudinal grade and horizontal curvature may affect RqSD, a 2-km road section was suggested and the SSD profile was calculated following the new algorithm. The road segment consists of a crest vertical curve superimposed with a simple horizontal curve. Given a $100-\mathrm{km} / \mathrm{h}$ design speed and $6 \%$ superelevation, a minimum radius of $437 \mathrm{~m}$ was calculated for the horizontal curve (Exhibit 3-15, AASHTO 2004). As for the crest curve, a minimum length of $624 \mathrm{~m}$ (i.e., $K=52$ ) was calculated assuming a $6 \%$ longitudinal grades for both curve approaches. Using a $2.5-\mathrm{sec}$ PR time and 0.29 friction coefficient, SDES was run and the RqSD profile was extracted as shown in Figure 4-3.

The figure indicates that a value of $182.00 \mathrm{~m}$ is required by drivers to stop on the upgrade part of the road whereas SSD increases to $262.20 \mathrm{~m}$ as the vertical grade changes over the crest curve. This huge difference (e.g., more than $80 \mathrm{~m}$ ) in RqSD values within few hundred meters of the road may indicate the inappropriateness of ignoring the influence of road geometry on RqSD. Compared to a value of $205.26 \mathrm{~m}$ for the approximate RqSD, the drivers would probably operate at a relatively high risk level that suggests the consideration of these current approximations.

As for the AASHTO formula, the same alignment was rechecked using $3.4 \mathrm{~m} / \mathrm{s}^{2}$ $(0.35 \mathrm{~g})$ deceleration rate. Figure $4-4$ indicates that a maximum difference of only $40.6 \mathrm{~m}$ may result between the RqSD on the straight upgrade and the curved downgrade parts of the alignment. This difference is less than the case of TAC formula since only the 
longitudinal grade is considered. It should be mentioned here that the suggested methodology described above will be used to estimate the exact RqSD thereafter.
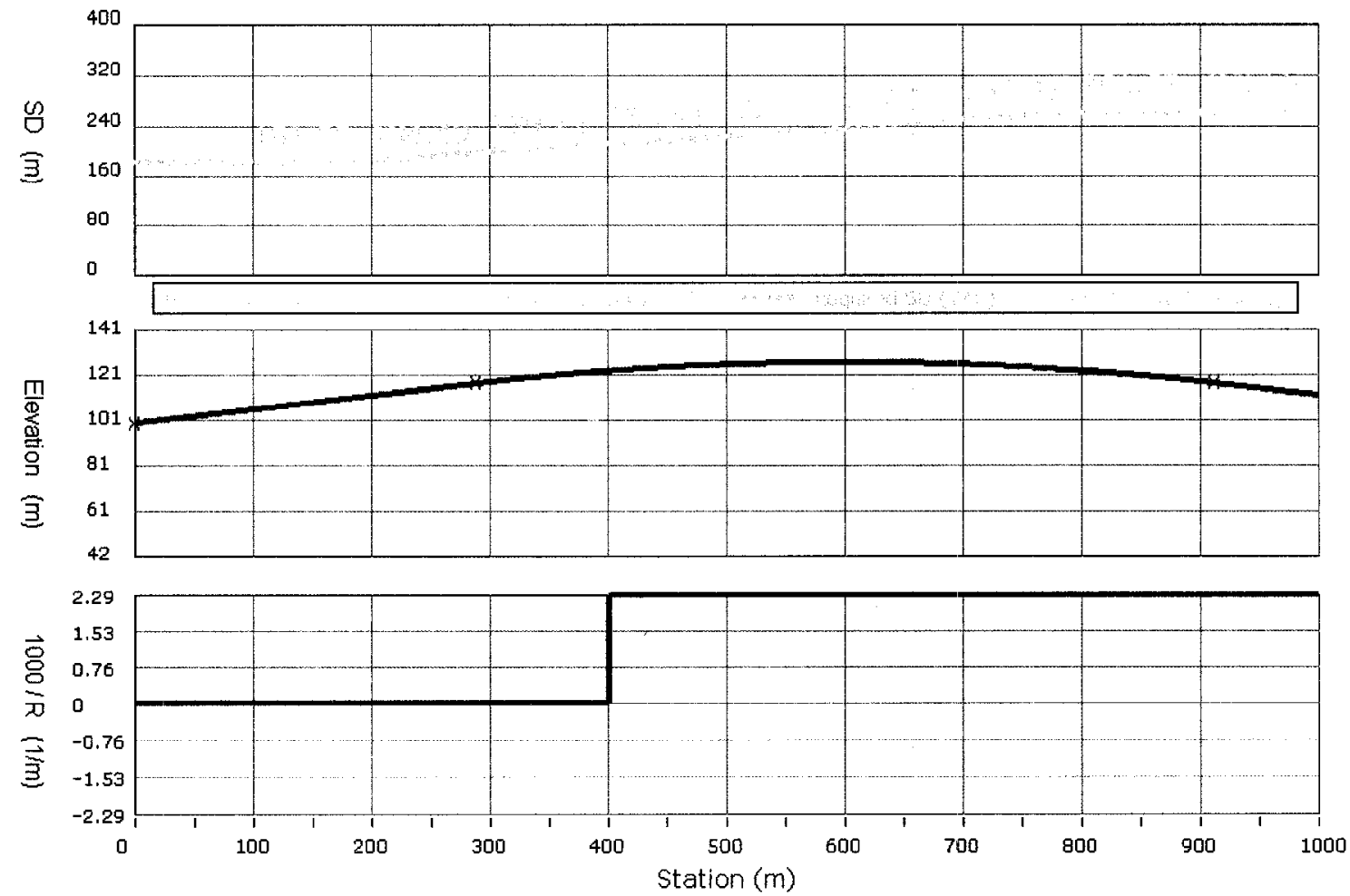

Figure 4-3: Influence of Longitudinal Grade and Horizontal Curvature on TAC RqSD.

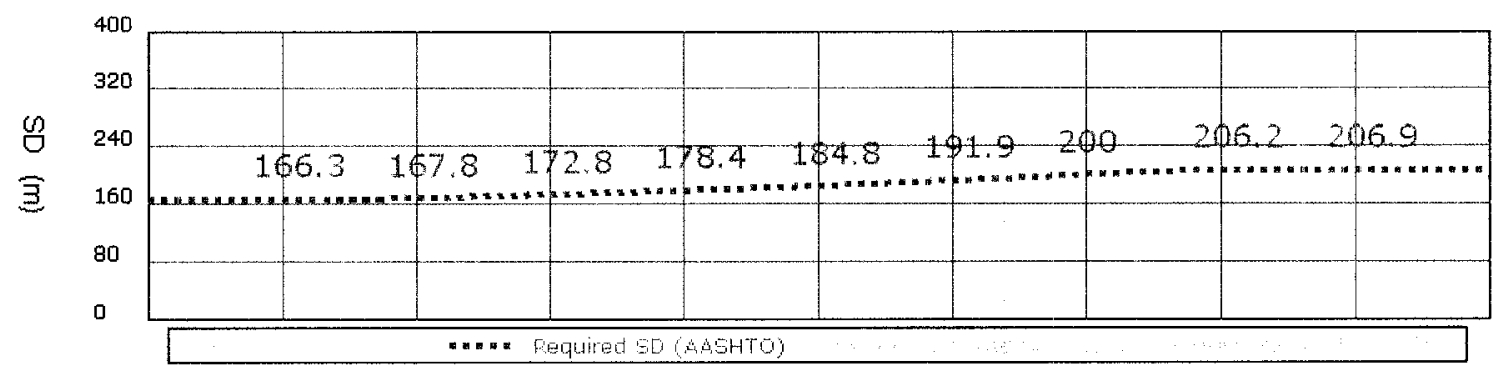

Figure 4-4: Influence of Longitudinal Grade on AASHTO RqSD. 


\subsection{Probabilistic versus Deterministic Approaches}

Over years, both TAC and AASHTO equations described earlier have been applied using the deterministic philosophy; an approach that utilizes a single near-worst value for each design parameter and yields a single conservative value for RqSD. This RqSD value has to be maintained along a specific roadway for acceptable designs. A road section that does not meet such requirement is considered as unsafe. On the other hand, the probabilistic approach can be followed by applying the basic principles described earlier in CHAPTER 3 and using either the simulation or analytical reliability techniques. The main advantage of this latter approach is that a design may be evaluated on a scale of appropriateness instead of the misleading notion of right and wrong design.

\subsubsection{Probabilistic Approach using Simulation Reliability Technique}

As mentioned before, the simulation technique relies on performing thousands of iterations in order to generate a RqSD's distribution. In each trial, individual parameter values are picked from pre-generated distributions of design parameters and SSD formulas are applied to simulate possible stopping scenarios. The application of the simulation technique in this section is presented utilizing the same example described earlier in Section 4.2.2. The 2-km alignment was entered to SDES to determine the probabilistic RqSD profile at $100-\mathrm{m}$ intervals. A total of 100,000 iterations were tried using different 100,000 pre-generated values of each design parameters. The parameters' distributions used for this purpose were taken from relevant studies in the literature as presented earlier in Section 2.4. 
For the distribution of PR time, a mean and standard deviation of 1.14 and $0.204 \mathrm{~s}$ were used to correspond to the most critical estimates reported by Fambro et al. (2000b). The same study was the source for the mean deceleration rate and standard deviation of $0.42 g\left(4.12 \mathrm{~m} / \mathrm{s}^{2}\right)$ and $0.06 \mathrm{~g}\left(0.59 \mathrm{~m} / \mathrm{s}^{2}\right)$. As for the operating speed, the two equations listed in the study by Andjus and Maletin (1998) were used.

$$
\begin{aligned}
& V 50=14.75 \ln R-11.69 \\
& S D=0.1085 V 50+0.99
\end{aligned}
$$

Equation 4-8

Equation 4-9

where; $V 50=$ mean operating speed $(\mathrm{km} / \mathrm{h}) ; R=$ horizontal curve radius $(\mathrm{m}) ;$ and $S D=$ standard deviation of operating speed $(\mathrm{km} / \mathrm{h})$.

Using a radius of $437 \mathrm{~m}$, a mean and standard deviation of 78 and $9.45 \mathrm{~km} / \mathrm{h}$ were calculated (see summary in Table 4-2). For simplicity, the same speed distribution was assumed along the entire segment. However, further adjustment is required to account for different speeds on tangents and curves as will be covered through the case study in CHAPTER 6. The means and standard deviations of all design parameters were then used to generate 100,000 values for each design parameter using Matlab. The generated numbers were then considered in calculating RqSD.

Table 4-2: Mean and Standard Deviation of Design Parameters.

\begin{tabular}{lcc}
\hline Parameter & Mean & Standard deviation \\
\hline Speed " $V$ " $(\mathrm{km} / \mathrm{h})$ & 78 & 9.45 \\
PR time " $t$ " $(\mathrm{sec})$ & 1.14 & 0.204 \\
Deceleration rate & $0.42 \mathrm{~g}$ & $0.06 \mathrm{~g}$ \\
\hline
\end{tabular}


It is also worthy to mention that the correlation between each pair of these independent variables should be considered in generating these random numbers. However, zero correlation coefficients were used in this example due to lack of information on such correlations. Further research, however, is required for future application of the proposed methodology. Figure 4-5 shows the SDES output that displays two profiles in the top part of the figure. The first profile represents the deterministic RqSD that used the near-worst design parameters $(V=100 \mathrm{~km} / \mathrm{h} ; t=2.5$ sec; and $a=0.35 g$ ) whereas the second profile connects the mean values of the RqSD distributions at each road section.

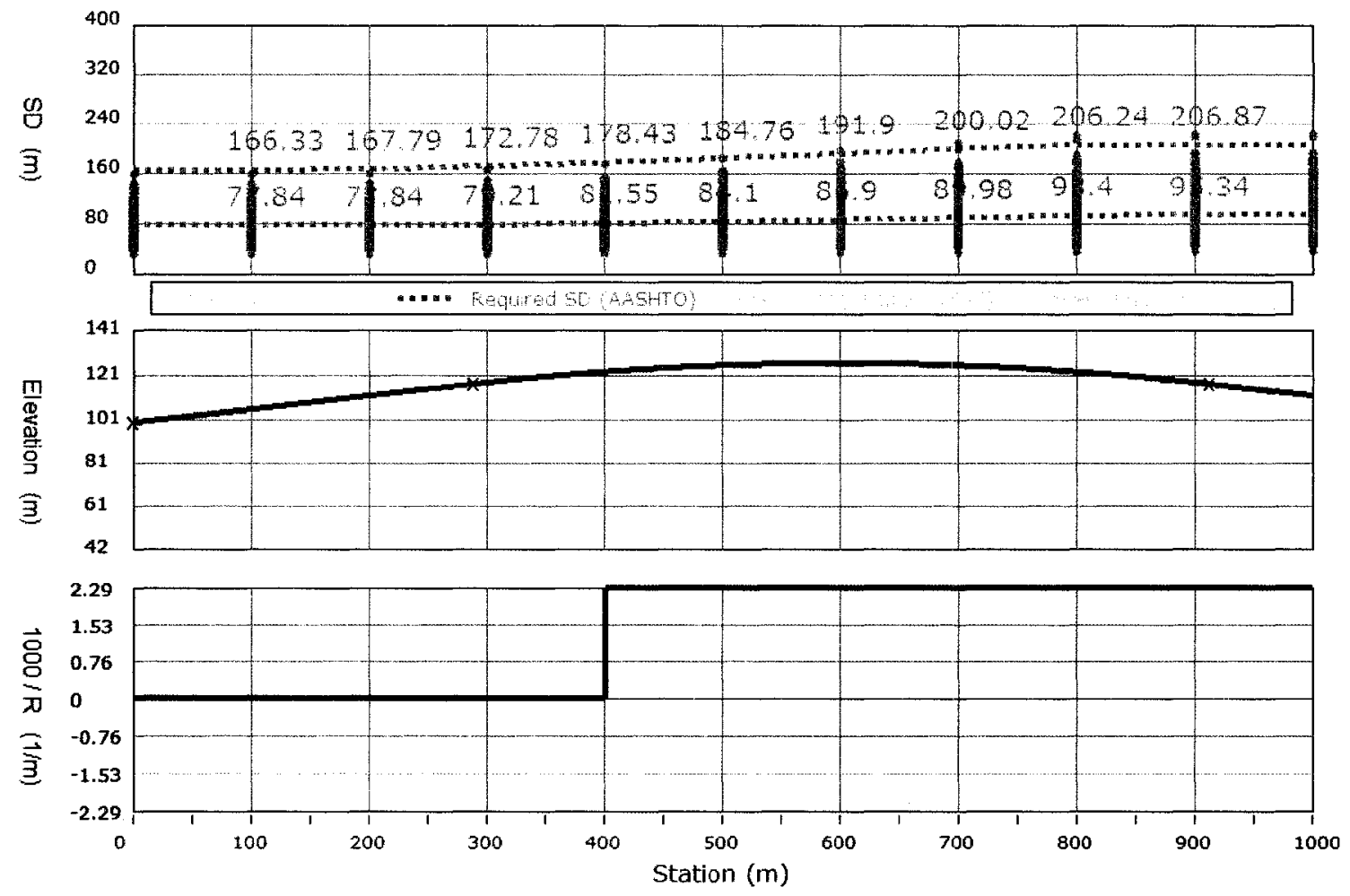

Figure 4-5: Deterministic versus Probabilistic AASHTO RqSD (100,000 iterations). 
Distributions are indicated in this graph by vertical lines formed by a marker for each of the 100,000 trials. It is obvious that the difference between the minimum and maximum values (i.e., 77.84 and $95.34 \mathrm{~m}$, respectively) of the probabilistic profile is less than the one of the deterministic profile (i.e., 166.33 and $206.87 \mathrm{~m}$, respectively). This is because, in the probabilistic approach, relatively larger values are used for the deceleration rate and this gives more capability to accommodate influences of longitudinal grades.

One can also notice that the deterministic RqSD value at each section is located near the upper limit of corresponding distribution. For more illustration, the distributions at Station 100 and Station 900 were extracted and plotted in Matlab. At Station 100, Only 18 drivers out of $100,000(0.018 \%)$ were acquiring higher RqSD than the deterministicbased value of $166.3 \mathrm{~m}$. As for Station 900 , a total of 126 drivers $(0.126 \%)$ were operating at potential risk with RqSD greater than the deterministic value of $206.9 \mathrm{~m}$. These very low percentages would indicate the extreme conservative nature of the current deterministic design that could result from the accumulated safety margins of contributed design parameters. 


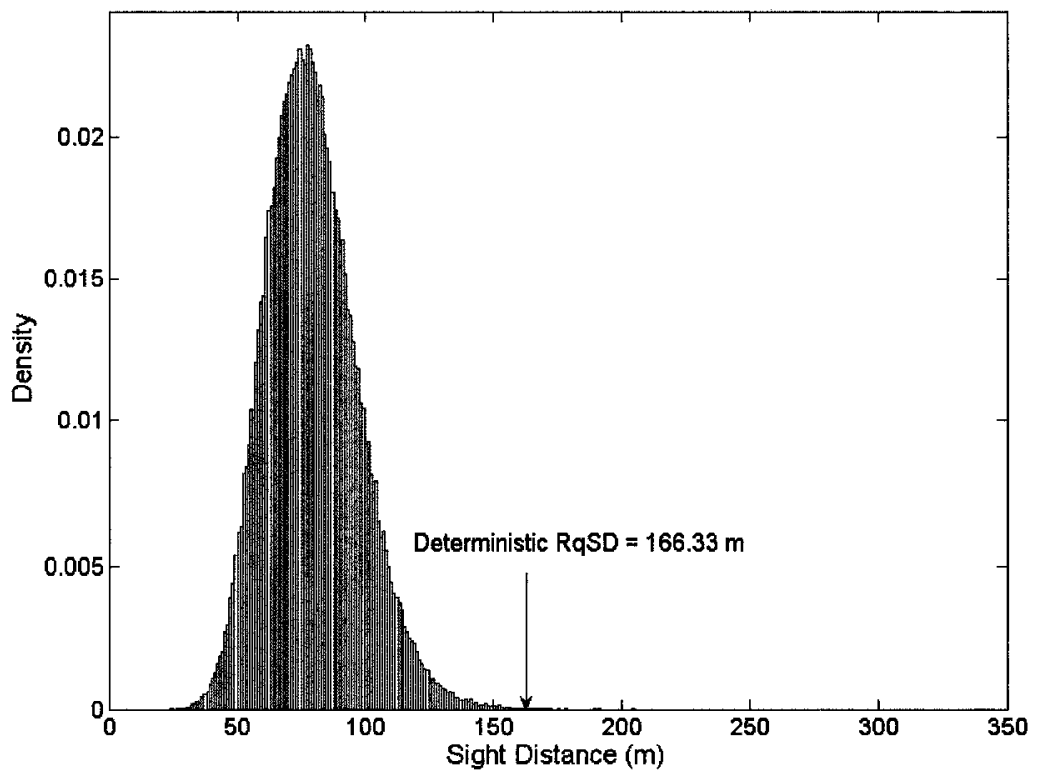

b) $\mathrm{RqSD}$ at Station 100

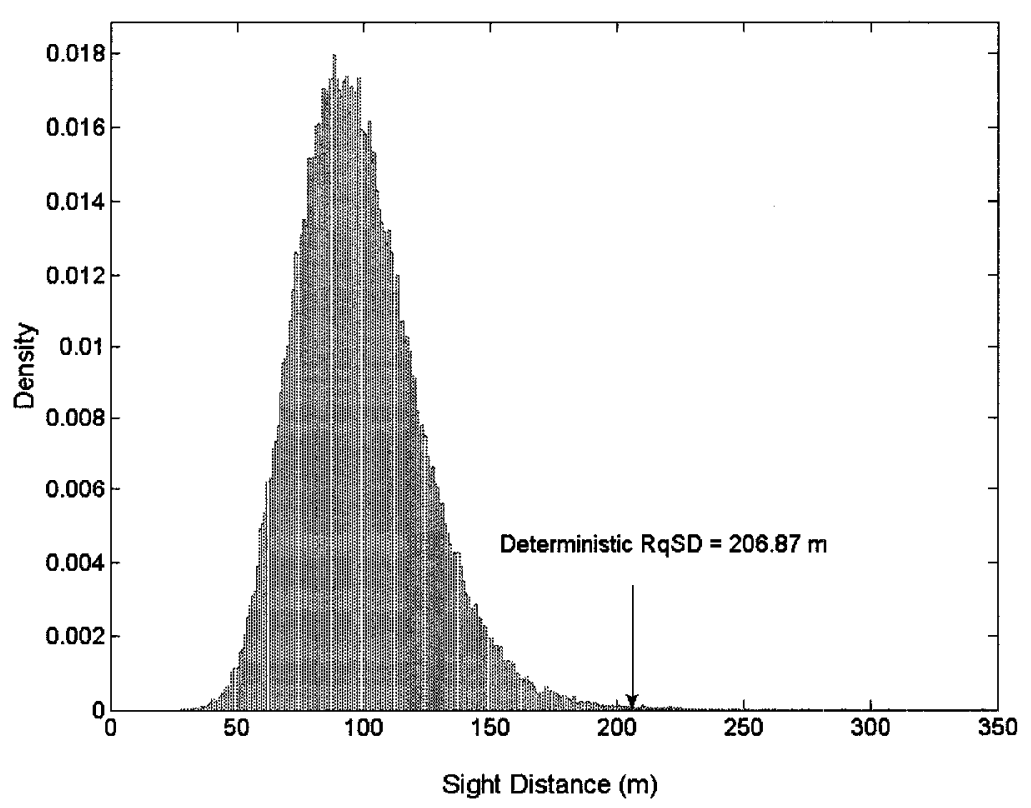

a) $\mathrm{RqSD}$ at Station 900

Figure 4-6: Deterministic versus Probabilistic AASHTO RqSD. 


\subsubsection{Probabilistic Approach using Analytical Reliability Technique}

With the fact that the AASHTO SSD formula gives as accurate RqSD as the simulated one on constant-grade road sections, the analytical technique may be applicable. This can be done by the common first-order reliability method. Using this method, the mean RqSD is estimated by substituting mean values of all design parameters whereas the variance can be expressed as follows:

$$
\begin{aligned}
\operatorname{var}(F)=\left(\frac{\partial F}{\partial V}\right)^{2} & \sigma_{V}^{2}+\left(\frac{\partial F}{\partial t}\right)^{2} \sigma_{t}^{2}+\left(\frac{\partial F}{\partial a}\right)^{2} \sigma_{a}^{2} \\
& +2\left(\frac{\partial F}{\partial V}\right)\left(\frac{\partial F}{\partial t}\right) \operatorname{cov}[V, t] \\
& +2\left(\frac{\partial F}{\partial V}\right)\left(\frac{\partial F}{\partial a}\right) \operatorname{cov}[V, a] \\
& +2\left(\frac{\partial F}{\partial t}\right)\left(\frac{\partial F}{\partial a}\right) \operatorname{cov}[t, a]
\end{aligned}
$$

Equation 4-10

$$
\text { where; } \begin{aligned}
& \frac{\partial \mathrm{F}}{\partial \mathrm{V}}=0.278 \mathrm{t}+\frac{\mathrm{V}}{127(\mathrm{a}+\mathrm{G})} \\
& \frac{\partial \mathrm{F}}{\partial \mathrm{t}}=0.278 \mathrm{~V} \\
& \frac{\partial \mathrm{F}}{\partial \mathrm{a}}=\frac{1}{254(\mathrm{a}+\mathrm{G})} \\
& \operatorname{cov}\left[\mathrm{x}_{\mathrm{i}}, \mathrm{x}_{\mathrm{j}}\right]=\rho_{\mathrm{x}_{\mathrm{i}}, \mathrm{x}_{\mathrm{j}}} * \sigma_{\mathrm{x}_{\mathrm{i}}} * \sigma_{\mathrm{x}_{\mathrm{j}}}
\end{aligned}
$$

Equation 4-11

Equation 4-12

Equation 4-13

Equation 4-14

where; $a=$ deceleration rate (gravity units).

It should be noted, however, that the analytical method is not valid when a stopping attempt is taking place in whole or in part on a variable-grade sections. This is because of the lack of explicit equations that would express such stopping scenario. 
Therefore, the simulation technique would be the only possible alternative. In addition, the equations listed above assume that the road surface condition permits deceleration rates at the choice of road users. At slippery conditions, the equations may underestimate the drivers need due to ignoring the friction actually available on the road.

To compare between the simulation and analytical reliability techniques, Equation 4-10 was used to calculate the standard deviation of the RqSD distribution at Station 100 . The rationale of this section is the fact that the vehicle is continuously occupying a constant-grade section while applying the vehicle's brakes. A standard deviation of $17.85 \mathrm{~m}$ had resulted from the simulation technique against $16.80 \mathrm{~m}$ using the analytical method. This difference of $1.05 \mathrm{~m}$ could be considered acceptable in relative terms and the simulation method was therefore considered satisfactory. It should be mentioned that the mean values using both the simulation and analytical techniques are the same as per previous discussion in Section 3.1.3.

\subsection{RqSD Considering both Deceleration and Friction}

It is obvious that both AASHTO and TAC SSD formulas are almost identical except for the use of deceleration rate $(a)$ in AASHTO versus friction coefficient $(F)$ in TAC. As mentioned earlier, the TAC SSD formula relies on the friction between the roadway surface and the vehicle tire whereas the AASHTO formula considers the deceleration rate that drivers would select at stopping manoeuvres. There has always been a debate on the appropriateness of each formula in highway geometric design. While TAC formula was criticized by Fambro (1997), McLean and Morrall (1998) mentioned that problems may arise in Canada from "low levels of friction provided when 
roads are iced-up in winter" (McLean and Morrall 1998); a problem that may be less pronounced in other countries such as USA.

In this research, however, the individual use of the two SSD formulas was considered inappropriate. On one side, the TAC formula relies on the friction coinciding with the fact that providing a higher friction would decrease RqSD. However, values of $F$ in TAC (1999), which are given in respect to design speed, are notably low as a conservative assumption. These friction values consider worst driving conditions (i.e., wet pavement) that the road surface is slippery enough to provide sufficient friction coefficients for vehicles attempting to stop. The approach, however, ignores the fact that, at such surface condition, road users may cautiously operate at speeds that are way lower than desired. Subsequently, the use of low friction values at relatively high speeds may not be very practical and, moreover, contradicts common driving behaviour. In addition, the use of high and near-real friction values as an alternative to the existing low values may result in impractical deceleration rates that could be hard to attain by most drivers.

On the other side, the use of deceleration rate (AASHTO formula) may seem more appropriate in the sense that drivers, in many instances, select their deceleration rates regardless of the surface characteristics. In general, one can state that deceleration rates may reflect a subjective human characteristic that "can be validated and defended as representative of the driving environment and safe driving behaviour" (Fambro (1997). However, there is a probability that a road surface fails to provide a sufficient frictional property required for such selected deceleration by a certain driver. 
Since the individual use of the AASHTO and TAC formulas could be described as inappropriate, a new methodology was suggested to account for both deceleration and friction. The methodology uses the deceleration rate as long as the road surface provides enough friction that meets such rate while the friction is used otherwise. In order to ensure the applicability of this assumption in SDES, a friction distribution is used as a controlling distribution. This friction distribution is set according to the prevailing weather and road surface condition. At dry surfaces, near-high friction values are probably available and a desired deceleration rate, as suggested in the literature, may hence be guaranteed. However, when a road surface is exposed to extensive rain or snow, friction values are often insufficient to support such deceleration rates. Accordingly, drivers may become exposed to high risk levels and, in many instances, lose control over their vehicle.

For more clarification, the consideration of friction in RqSD determination is illustrated in Figure 4-7 where, at each trial of the probabilistic iterations, two values are picked in sequence from both deceleration and friction distributions. If the selected friction value is greater than the deceleration value, the AASHTO formula is used. Contradictory, TAC formula is more suitable when the picked friction value is lower than the deceleration value indicating the case with slippery road surfaces. This process is repeated for $N$ times allowing a more realistic consideration of the surface conditions. More discussion about friction distributions based on field measurements will be presented in Section 4.5. In addition, the application of the methodology will be presented through a case study later in CHAPTER 6. 


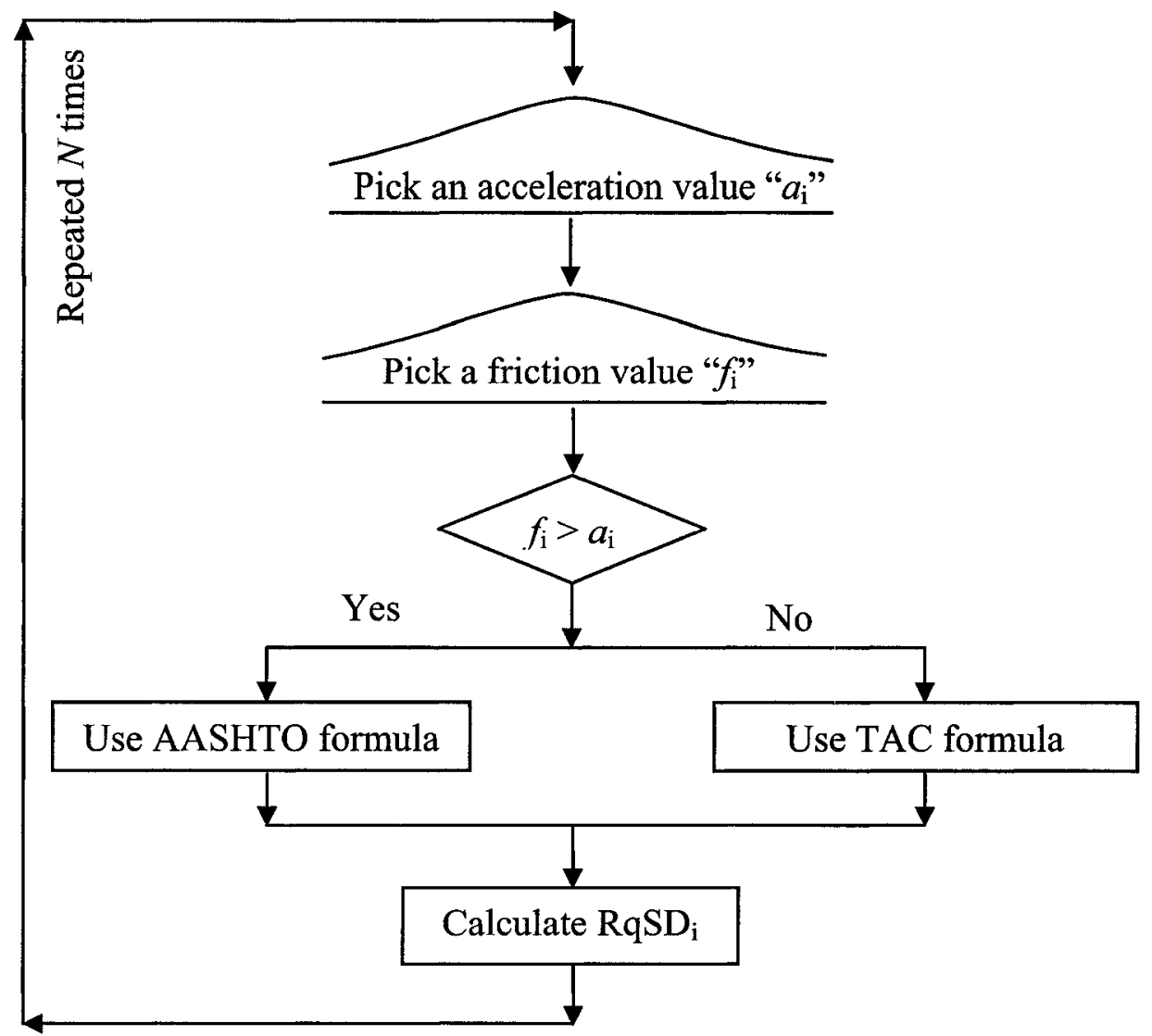

Figure 4-7: New Methodology to Calculate the RqSD Distribution using Both Deceleration and Friction.

\subsection{Field Measurement of Friction Coefficient}

Since the evaluation of the friction coefficient between the pavement and vehicles' tyres is essential to ensure safe operations at stopping attempts, friction data were collected on various highway sections around the City of Ottawa. The work presented in this section is based in part on a project funded by the City of Ottawa to evaluate the change in the friction properties with time (Jamil 2005). Another data set was further collected for the purpose of this research. 


\subsubsection{T-10 Friction Trailer}

The equipment used for friction data collection is the T-10 Continuous Friction Measuring Equipment (CFME) available in Carleton University's laboratory. As shown in Figure 4-8, CFME is a trailer that houses an additional wheel with a $15 \%$ slip angle. Both the vertical and horizontal forces on the wheel are recorded and sent to a special computer to process the data and produce a continuous profile of friction. In addition, Figure 4-9 shows the passenger minivan that has been fitted to house a water tank connected to the trailer such that water can be sprayed at a specified rate in front of the measuring wheel to maintain a wet pavement condition during the measurements.

The user can also deactivate the water to simulate a stopping attempt on a dry surface condition. It should be noted that by having the slip angle, the measured friction is consistent with driver behaviour, as drivers have been found to modulate their braking in order to maintain steering control (Olson et al. 1984). In contrast, a fully-locked wheel, which is used by some agencies for measuring skid resistance, represents an unrealistic driver action as drivers would lose directional control if the vehicle's wheels are allowed to fully lock.

\subsubsection{Data Collection}

In general, the friction is a function of many factors among which the road surface condition is a key factor. While dry surfaces are capable of delivering high friction that is normally needed for a proper vehicle control, wet surfaces may cause a significant reduction in friction and hence become unsafe. 

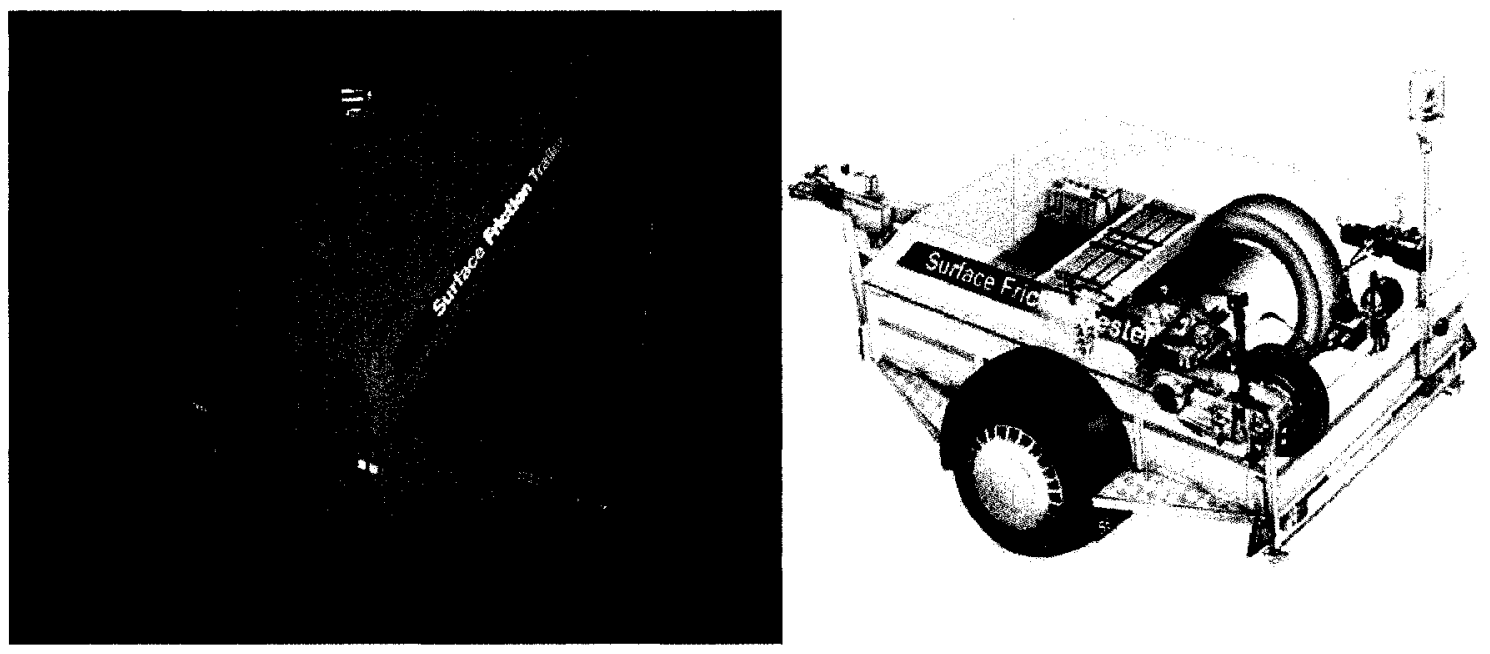

Figure 4-8: CFME T-10 Trailer for Continuous Friction Measurement.
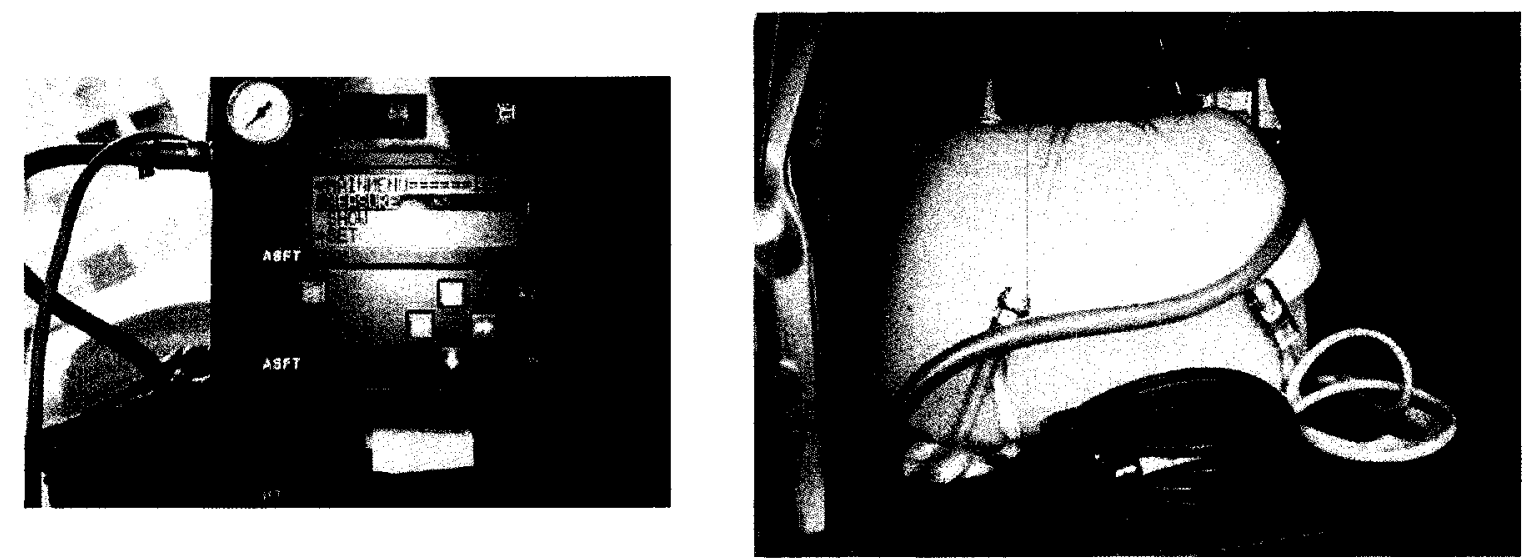

Figure 4-9: CFME Computer Water Tank Fitted in the Minivan.

In this research, the friction was evaluated at four different situations simulating four different driving scenarios including (1) dry surface in summer, (2) dry surface in winter, (3) wet surface in summer, and (4) snowy surface in winter. In addition, the variation of pavement type, temperature, and operating speed recorded during data collection should implicitly consider the contribution of such factors. A total of 11 
locations were selected with new asphalt concrete overlays in the vicinity of the City of Ottawa to encompass different mix types. The objective was to initiate a database for friction history of different pavement mixes and monitor the change in friction characteristics due to traffic and environmental conditions. Table 4-3 represents a total of eight roads with 5 different pavement types. Except the case of snowy surface in winter, the table shows the construction date as well as the date/age of each of three attempts of data collections.

Table 4-3: Locations and Dates for Data Collection.

\begin{tabular}{|c|c|c|c|c|c|}
\hline Road & $\begin{array}{l}\text { Pavement Type } \\
\text { (PG) }\end{array}$ & $\begin{array}{c}\text { Construction } \\
\text { Date }\end{array}$ & $\begin{array}{l}\text { Test } 1 \\
\text { (Age) }\end{array}$ & $\begin{array}{l}\text { Test } 2 \\
\text { (Age) }\end{array}$ & $\begin{array}{l}\text { Test } 3 \\
\text { (Age) }\end{array}$ \\
\hline $\begin{array}{c}\text { Highway } \\
174\end{array}$ & $\begin{array}{c}12.5 \mathrm{~mm} \text { SMA } \\
\text { Level C (70-34) }\end{array}$ & $15 / 08 / 2004$ & $\begin{array}{c}16 / 08 / 2004 \\
\text { (1 Day) }\end{array}$ & $\begin{array}{c}19 / 07 / 2005 \\
\text { (1 Year) }\end{array}$ & $\begin{array}{c}\text { 24/12/2007 } \\
\text { (3Years) }\end{array}$ \\
\hline $\begin{array}{c}\text { Second } \\
\text { Line Road }\end{array}$ & HL3 (58-34) & $18 / 09 / 2004$ & $\begin{array}{r}20 / 09 / 2 \\
(2 \mathrm{Day}\end{array}$ & $\begin{aligned} 20 / 07 / \\
(1 \mathrm{Yg}\end{aligned}$ & NA \\
\hline $\begin{array}{l}\text { Old Carp } \\
\text { Road }\end{array}$ & HL3 (58-34) & $20 / 09 / 2004$ & $\begin{array}{l}07 / 10 / \\
(17 \mathrm{D}\end{array}$ & $\begin{array}{r}20 / 07 \\
(1 \mathrm{Y} \\
\end{array}$ & NA \\
\hline $\begin{array}{c}\text { Devine } \\
\text { Road }\end{array}$ & HL3 (58-34) & $01 / 09 / 2004$ & $\begin{array}{c}07 / 09 / 2004 \\
\text { (6 Days) }\end{array}$ & $\begin{array}{r}24 / 07) \\
(1 \mathrm{YG}\end{array}$ & $\begin{array}{c}\text { 24/12/2007 } \\
\text { (3Years) }\end{array}$ \\
\hline $\begin{array}{l}\text { Fortune } \\
\text { Drive }\end{array}$ & HL3 (58-28) & $13 / 08 / 2004$ & $\begin{array}{c}25 / 08 / 2004 \\
\text { (12 Days) }\end{array}$ & $\begin{array}{c}19 / 07 / 2005 \\
(1 \text { Year) }\end{array}$ & $\begin{array}{c}24 / 12 / 2007 \\
\text { (3Years) }\end{array}$ \\
\hline \multirow{2}{*}{$\begin{array}{c}\text { Upper } \\
\text { Dwyer Hill } \\
\text { Road }\end{array}$} & HL3 (58-34) & \multirow{2}{*}{$28 / 09 / 2004$} & \multirow{2}{*}{$\begin{array}{c}\text { 07/10/2004 } \\
\text { (9 Days) }\end{array}$} & $\begin{array}{c}20 / 07 / 2005 \\
(1 \text { Year })\end{array}$ & \multirow{2}{*}{ NA } \\
\hline & $\begin{array}{c}12.5 \mathrm{~mm} \text { SP Level } \\
\text { A (58-34) }\end{array}$ & & & NA & \\
\hline $\begin{array}{c}\text { Fallowfield } \\
\text { Road }\end{array}$ & $\begin{array}{l}\text { Microsurfacing } \\
\text { Type II }\end{array}$ & $30 / 06 / 2004$ & $\begin{array}{c}16 / 08 / 2004 \\
\text { (47 Days) }\end{array}$ & $\begin{array}{c}20 / 07 / 2005 \\
\text { (1 Year) }\end{array}$ & $\begin{array}{c}24 / 12 / 2007 \\
\text { (3Years) }\end{array}$ \\
\hline $\begin{array}{c}\text { Prince of } \\
\text { Wales } \\
\text { Drive }\end{array}$ & $\begin{array}{c}\text { 12.5FC1 SP Level } \\
\text { D (64-34) }\end{array}$ & $27 / 09 / 2004$ & $\begin{array}{c}07 / 10 / 2004 \\
\text { (10 Days) }\end{array}$ & $\begin{array}{c}24 / 07 / 2005 \\
\text { (1 Year) }\end{array}$ & $\begin{array}{c}24 / 12 / 2007 \\
\text { (3Years) }\end{array}$ \\
\hline
\end{tabular}

where; SMA $=$ stone mastic asphalt and $\mathrm{HL}=$ hot load. 
Test 1 and 2 in this table were conducted in the summer/fall of 2004 and 2005, respectively. While Test 2 was carried out with water being activated in CFME to simulate worst driving condition in summer, Test 1 was performed at both dry and wet surface conditions. In addition, Test 3 was carried out on only 5 roads during winter time, where the road surface was fairly dry with temperature approaches $-10^{0} \mathrm{C}$ (see Figure 4-10-a). During this test, the water was deactivated in CFME since the use of water at low temperatures was going to create an ice film that is hazardous for traffic operations. The purpose of measuring friction in winter was to complement the already existing records and hence give a wider picture of the friction variation for all seasons in the year. Furthermore, another dataset was conducted on a road section located on Devine Road where the snow was backed up (see Figure 4-10-b). This section corresponds to an aftersnow event where the road surface has not been cleared. Data collected at such surface condition would represent the worst driving scenario compared to the other three surface conditions considered in this research.

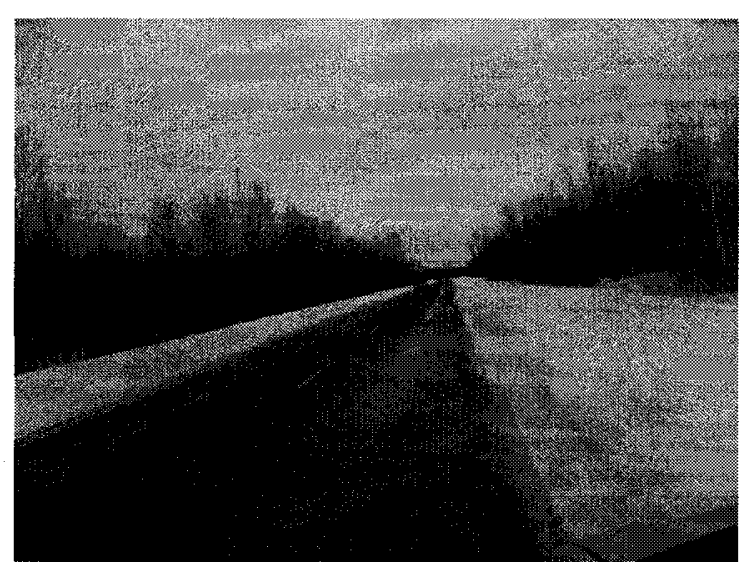

a) Dry Surface in Winter

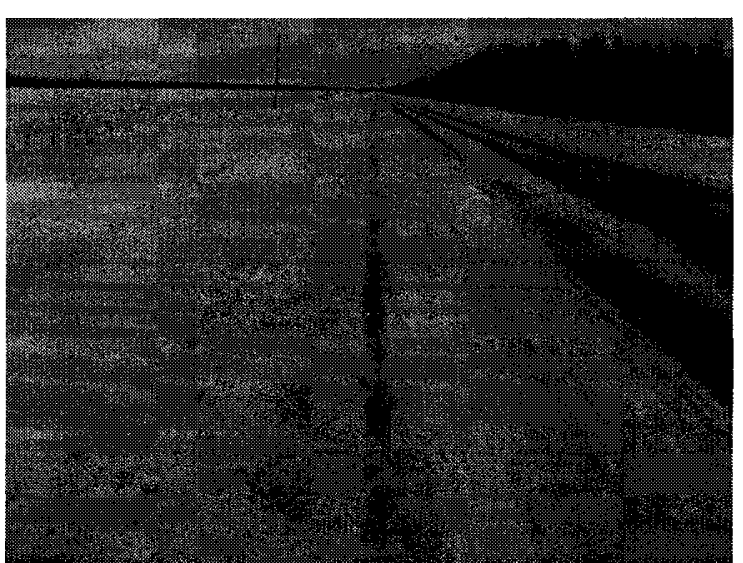

b) Snow-Backed Surface in Winter

Figure 4-10: Snow and Dry Surface Conditions during Winter Time. 
It should also be mentioned that the actual measurements in the field targeted the wheel path rather than the middle of the lane to ensure that the measured friction is representative of what is available for the motorists and vehicles. Calibrations of the CFME load and friction were carried out in the beginning of everyday of data collection. Initially, the data were collected for three runs on each road section to check the repeatability of the results with each run covering a distance from 200 to $500 \mathrm{~m}$. However, the results were found to be highly repeatable and subsequently only one run of testing was conducted for each road section for the rest of the experiment.

\subsubsection{Descriptive Analysis and Findings}

Table 4-4 shows the descriptive analysis of all data collected including the total length of road sections, speed, and friction coefficient. The table shows that the friction was measured on a total length of 7.8 and $18.0 \mathrm{~km}$ for dry and wet surface conditions in the summer, respectively. On the other side, a total of $2.2 \mathrm{~km}$ was used during winter time with the difficulty to collect further data due to hazardous surface conditions. Even though, the data were considered fairly capable of describing the variation of friction characteristics. Given that a reading is provided every almost $10 \mathrm{~m}$ representing an average friction over such length, the collected dataset contains hundreds of values that were used to predict four friction distributions as shown in Figure 4-11.

The figure shows a total of four distributions for the four different driving scenarios outlined earlier. The figure also shows one deceleration distribution generated in gravity units with a mean and standard deviation of $0.42 \mathrm{~g}$ and $0.06 \mathrm{~g}$, respectively. This deceleration distribution may describe a desired deceleration rate by most drivers 
(Fambro et al. 2000b). It is obvious that the friction values collected at dry surface condition in both summer and winter are the closest among the five distributions. However, the ANOVA analysis indicated that the two distributions are significantly different at $95 \%$ confidence level.

Table 4-4: Descriptive Analysis of Speed and Friction.

\begin{tabular}{lcccccccc}
\hline \multirow{2}{*}{ Season } & \multirow{2}{*}{ Condition } & \multirow{2}{*}{$\begin{array}{c}\text { Length }(\mathrm{km}) \\
\text { [\# of values] }\end{array}$} & \multicolumn{2}{c}{ Speed $(\mathrm{km} / \mathrm{h})$} & \multicolumn{4}{c}{ Friction Coefficient } \\
\cline { 4 - 9 } & & mean & $\sigma$ & mean & $\sigma$ & min. & max. \\
\hline \multirow{2}{*}{ Summer } & Dry & $7.8[785]$ & 45.03 & 6.40 & 0.90 & 0.04 & 0.73 & 1.01 \\
& Wet & $18.0[1864]$ & 59.21 & 16.33 & 0.73 & 0.10 & 0.38 & 1.03 \\
\hline \multirow{2}{*}{ Winter } & Dry & $2.0[206]$ & 76.85 & 12.61 & 0.92 & 0.05 & 0.72 & 1.03 \\
& Snow & $0.2[23]$ & 58.27 & 2.0 & 0.26 & 0.05 & 0.21 & 0.37 \\
\hline
\end{tabular}

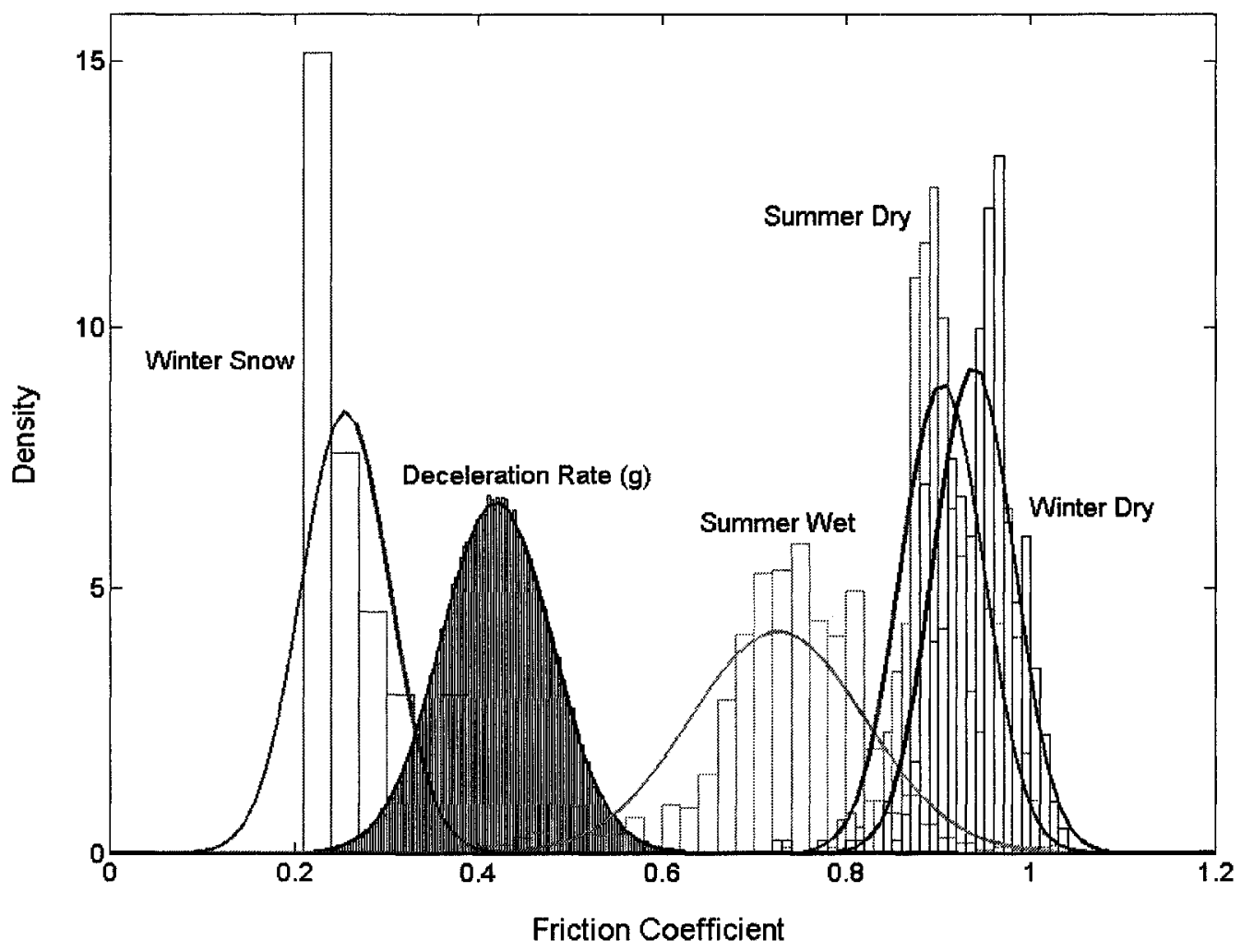

Figure 4-11: Distributions of Friction Coefficient for Different Driving Scenarios. 
In addition, the minimum friction value in these two distributions $(0.72)$ was found even greater than the highest value in the deceleration distribution $(0.67 \mathrm{~g})$. In other words, the deceleration distribution does not intersect with any of these two friction distributions collected on dry surfaces. This may support the hypothesis that the dry road surface would provide a sufficient friction for drivers to select a deceleration rate at their choice. Since these two distributions would provide a safe driving environment in respect to the considered deceleration distribution, the two distributions were merged together forming one distribution with a mean of 0.907 and standard deviation of 0.045 . This distribution was considered a representative of dry surface conditions during the entire year.

On the other side, the friction distribution in summer at wet surface condition was found overlapping the deceleration distribution indicating a possibility of having drivers demanding higher friction than actually provided. Such a scenario might induce a certain degree of hazard that can be quantified by the percentage of those drivers to the whole sample size in a specific simulation attempt. The last distribution in this graph represents the friction in winter for snowy surfaces. The extreme low values of this distribution reflect the great risk of speeding on slippery surfaces. The distribution also indicates that more than $50 \%$ of drivers would operate at risk with insufficient friction.

In SDES, the friction distribution, which is required for the methodology presented earlier in Section 4.4, is formed of three parts. Each part represents a road surface condition including dry, wet, and snow. The percentages of these parts in the distribution depend on the prevailing weather condition in the vicinity of the road under 
study. In addition, each part is formed using the corresponding mean and standard deviation outlined above. A distribution formed using such approach may help provide a close description of frication over a specific road surface.

\subsubsection{Relationship between Speed and Friction}

Both the simulation and analytical reliability techniques allow the inclusion of correlations between individual design parameters to describe the performance function. While these correlations are part of the equation that describes the variance in the analytical method, the simulation technique may consider such correlations in generating the parameters' distributions. As mentioned before, there are four factors influencing the RqSD including speed, PR time, acceleration, and friction. Given the lack of information about such correlations in the literature, an attempt was made to utilize the CFME friction trailer to evaluate the correlation between speed and friction.

In this regard, a road section on Devine Road was selected to measure friction at 5 different speeds including $40,60,80,100$, and $120 \mathrm{~km} / \mathrm{h}$. Each run was performed on a length of $450 \mathrm{~m}$ except the case of $120 \mathrm{~km} / \mathrm{h}$ where a length of only $200 \mathrm{~m}$ was used for safety reasons. The average friction values were plotted against speed. In addition, the recommended friction values by TAC (1999) were also plotted as shown in Figure 4-12. The trend of the collected field measurements indicated a reduction in friction with the increase in operating speed; a trend that agrees with the assumption in TAC (1999). Moreover, the correlation between the speed and friction on a total of 195 collected values was found to be -0.28 . 


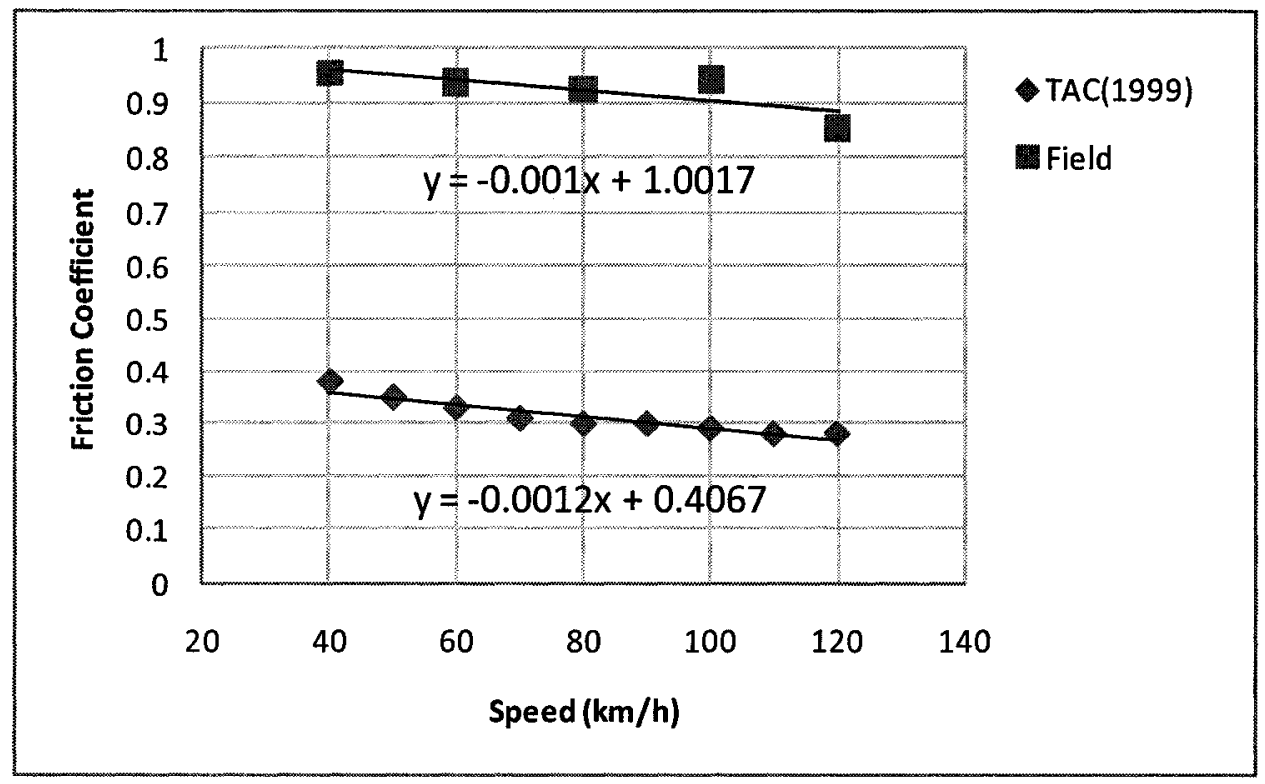

Figure 4-12: Relationship between Friction and Speed.

\subsection{Summary}

In this chapter, a suggested algorithm was developed and programmed to describe RqSD considering the continuous change in the longitudinal grade of road alignments. Possible reduction in longitudinal friction was also taken into account. Detailed methodology was further demonstrated to draw the RqSD profile along a certain road segment. In addition, the individual use of the AASHTO and TAC formulas to calculate RqSD was considered inappropriate. Rather, a new approach was suggested that considers both formulas. Moreover, the difference between the deterministic and probabilistic approaches to calculate RqSD was also highlighted showing the conservative nature of current practice. Finally, field measurements of friction were collected to assess the friction variation at different road surface conditions. The relationship between speed and friction was also investigated. 


\section{CHAPTER 5}

\section{Probabilistic Available Sight Distance}

In this chapter, different approaches to calculate the available sight distance (AvSD) will be presented. Section 5.1 provides a general discussion and description of different types of AvSD. In Section 5.2, checks are conducted to test the ability and precision of SDES to meet the traditional analytical formulas. This step was performed on only $2 \mathrm{D}$ design approaches with the lack of closed-form solutions of 3D AvSD. In addition, Section 5.3 uses an application example to highlight the difference between the probabilistic and deterministic design approaches. Moreover, the difference between 2D and 3D AvSD using the concept of the reliability theory is discussed in Section 5.4.

\subsection{Introduction to 2D and 3D AvSD}

The available sight distance (AvSD) can be defined as the distance that a driver would actually see ahead at a certain road section. As mentioned before, this distance was formulated in current design guides utilizing separate horizontal and vertical road projections; namely in this research as 2D-H and 2D-V AvSD, respectively. On one hand, a lateral obstruction like a building or upward side slope on a horizontal curve could limit the visibility of objects located along the centerline of the inner lane to only 2D-H AvSD. Given that the obstruction is located at a horizontal sight offset (HSO) from the 
centerline, a formula was presented in both AASHTO (2004) and TAC (1999) to relate $H S O$ to $2 \mathrm{D}-\mathrm{H} \mathrm{AvSD}$ and minimum curve radius $(R)$.

$$
H S O=R\left[1-\cos \frac{28.65 * S}{R}\right]
$$

where; $S=2 \mathrm{D}-\mathrm{H}$ AvSD; $R=$ curve radius; $H S O=$ horizontal sightline offset.

After arranging the terms in Equation 5-1, 2D-H AvSD can be expressed as a function of the curve radius and other cross-section elements such as lane, shoulder, and side slope. Assuming a simple cross-section with only one side slope as shown in Figure 5-1, AvSD can be formulated as follows:

$$
\begin{aligned}
& S=\frac{R}{28.65} \cos ^{-1}\left[1-\frac{H S O}{R}\right] \\
& H S O=\frac{L W}{2}+S h W+\left[\frac{h_{1}+h_{2}}{2}+\frac{L S * L W}{2}+S h S * S h W\right] S S
\end{aligned}
$$

where; $h_{1}=$ driver eye height; $h_{2}=$ object height; $L W=$ lane width; $S h W=$ shoulder width; $L S=$ lane cross slope $=S h S=$ shoulder cross slope; $S h W=$ shoulder width; and $S S=$ side slope (horizontal : vertical).

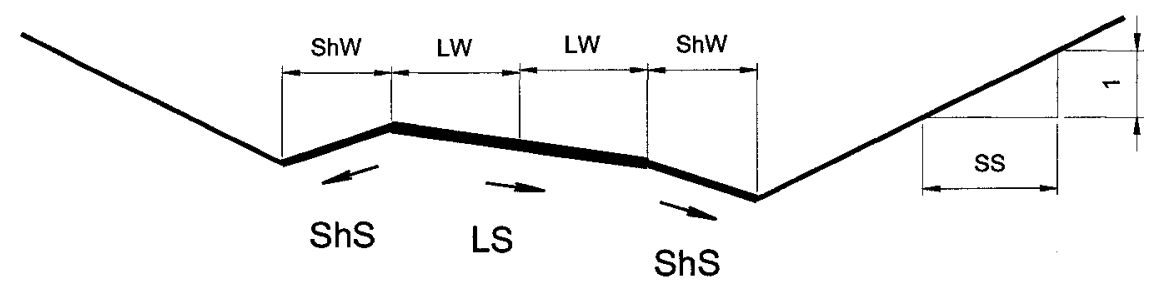

Figure 5-1: A Simple Cross-Section at the Midpoint of a Horizontal Curve. 
On the other hand, 2D-V AvSD is the distance calculated considering only the vertical projection of the alignment. The method, as presented in current design guides, assumes that the vertical curvature of the road surface may cause a driver with eye height $h_{1}$ to see an objects with height $h_{2}$ only 2D-V AvSD ahead along the vehicle path. For design purposes, two formulas were listed in both TAC (1999) and AASHTO (2004) to calculate a suitable length of a crest vertical curve in terms of $h_{1}$ and $h_{2}$.

$$
\begin{aligned}
& L_{c}=2 S-\frac{200\left(\sqrt{h_{1}}+\sqrt{h_{2}}\right)^{2}}{A} \quad ; S>L_{c} \\
& L_{c}=\frac{A * S^{2}}{200\left(\sqrt{h_{1}}+\sqrt{h_{2}}\right)^{2}} \quad ; S<L_{c}
\end{aligned}
$$

where; $S=2 \mathrm{D}-\mathrm{V}$ AvSD, $A=$ algebraic difference in longitudinal grades, $h_{1}=$ driver eye height, $h_{2}=$ object height.

The 2D-H AvSD can again be expressed by reformatting the terms in the equation above as follows:

$$
S=10\left[\sqrt{\frac{2 h_{1} L_{c}}{A}}+\sqrt{\frac{2 h_{2} L_{c}}{A}}\right]
$$

Equation 5-6

In the real world, it is seen frequently that horizontal curves are superimposed with vertical ones forming compound curves that do not belong solely to only one of the two approaches described above. By following the current practice in this case, the two values of $2 \mathrm{D}-\mathrm{H}$ and $2 \mathrm{D}-\mathrm{V}$ AvSD have to be checked to decide which of them is more critical. This process was programmed in SDES and such critical value was denoted here 
as 2D-C AvSD. It should be also noted that this step was helpful to test the difference between the $2 \mathrm{D}$ and $3 \mathrm{D}$ AvSD profiles of compound curves, as will be presented later in this chapter. Besides the three types of 2D AvSD mentioned above, 3D AvSD will also be used to describe such distance covered by a driver's eye considering the $3 \mathrm{D}$ nature of the highway. As discussed before, this distance is calculated by SDES utilizing a mesh that fits the road surface and then checking the intersection of the sight line, with different lengths, with the elements of this mesh (see Figure 5-2). The 3D AvSD is more realistic than 2D AvSD, which may underestimate or overestimate the actual road supply. It should also be emphasized that SDES has the advantage to describe the AvSD profile along the entire road segment rather than giving a single value using the analytical approach.

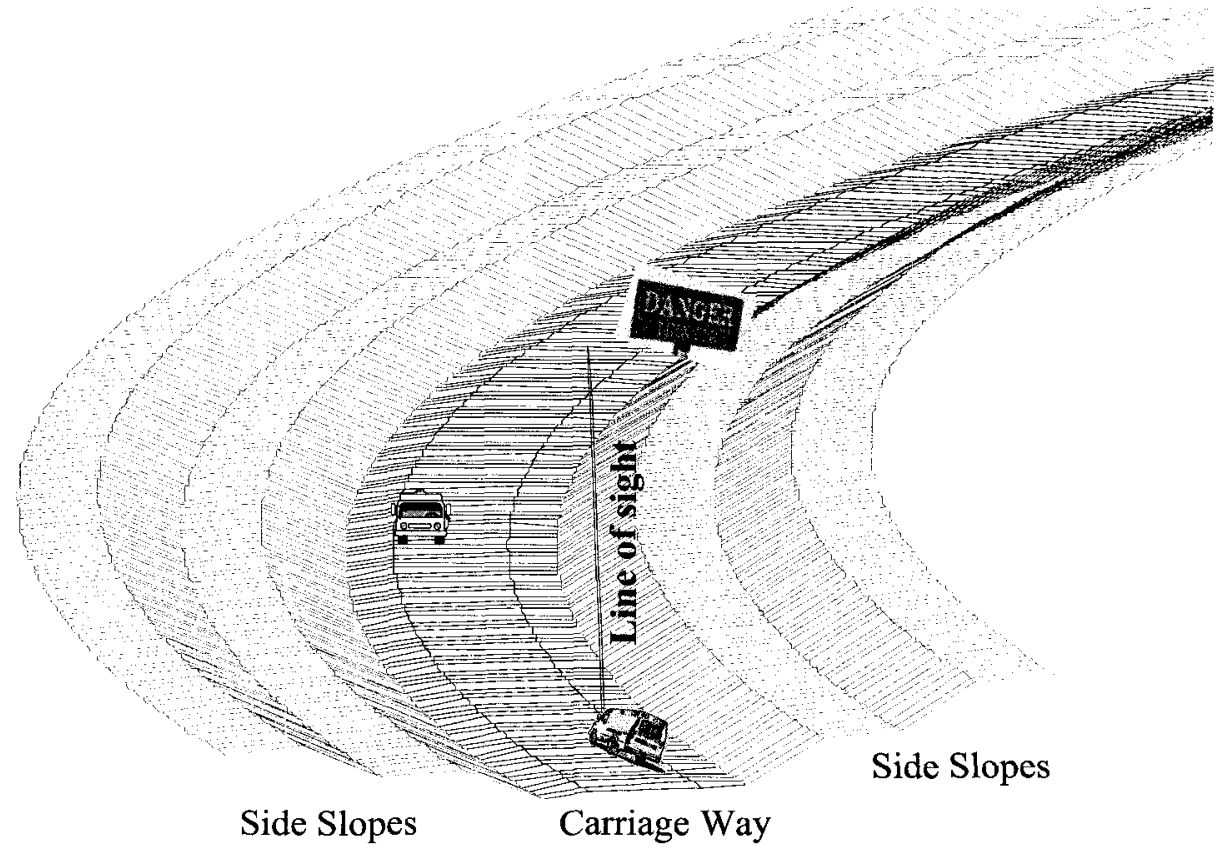

Figure 5-2: Calculation of 3D AvSD. 


\subsection{SDES Simulation and Precision}

Before running SDES, it was necessary to check the appropriateness of the recoded program after the conversion to Visual Basic.Net. Same example used before in Section 4.2.2 was reused for this purpose. However, the cross-section was further customized using a 3.8-m lane width, $0.06 \%$ superelevation, 5-m shoulder width, $0.08 \%$ shoulder slope, and 2H:1V side slope (see Figure 5-3). This step was not necessary in CHAPTER 4 since RqSD is not function of the cross sectional elements. Using values of 1.08 and $0.6 \mathrm{~m}$ for $h_{1}$ and $h_{2}$, respectively, and substituting back in Equation 5-2 and Equation 5-3, a minimum radius of $436.34 \mathrm{~m}$ was obtained to satisfy the sight distance design basis. This AvSD-based radius was thought to be close enough to the stabilitybased radius calculated before (e.g., $437 \mathrm{~m}$ ). In addition, a vertical curve length of $624 \mathrm{~m}$ remains the same as previously estimated. In the following two subsections, $2 \mathrm{D}-\mathrm{V}$ and 2D-H AvSD obtained from SDES for a vertical-only and horizontal-only alignments, respectively, will be checked against values resulting from the formulas presented earlier.

\subsubsection{AvSD on Vertical-Only Alignment}

There are two ways SDES can be operated to calculate 2D-V AvSD; (1) by using the $2 \mathrm{D}$ finite element to simulate the $2 \mathrm{D}$ analytical formulas and (2) by creating a $3 \mathrm{D}$ mesh that fits the road surface and side slopes for a vertical-only road segment and then simulating a 3D driving scenario. It should be noted that, by inputting the horizontal alignment as one straight segment, the second approach still describes 2D-V AvSD even though the road surface is created in 3D. Following the two approaches, the two AvSD profiles were found identical to the one shown in Figure 5-4. 


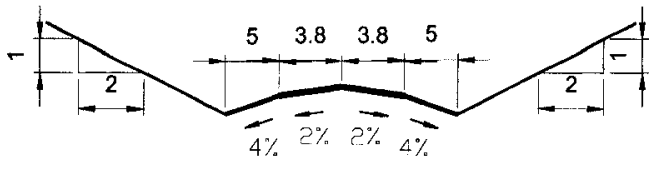

$\mathrm{X}$-Section at Tangent

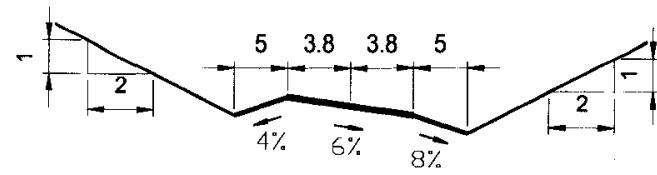

$\mathrm{X}$-Section at Curve

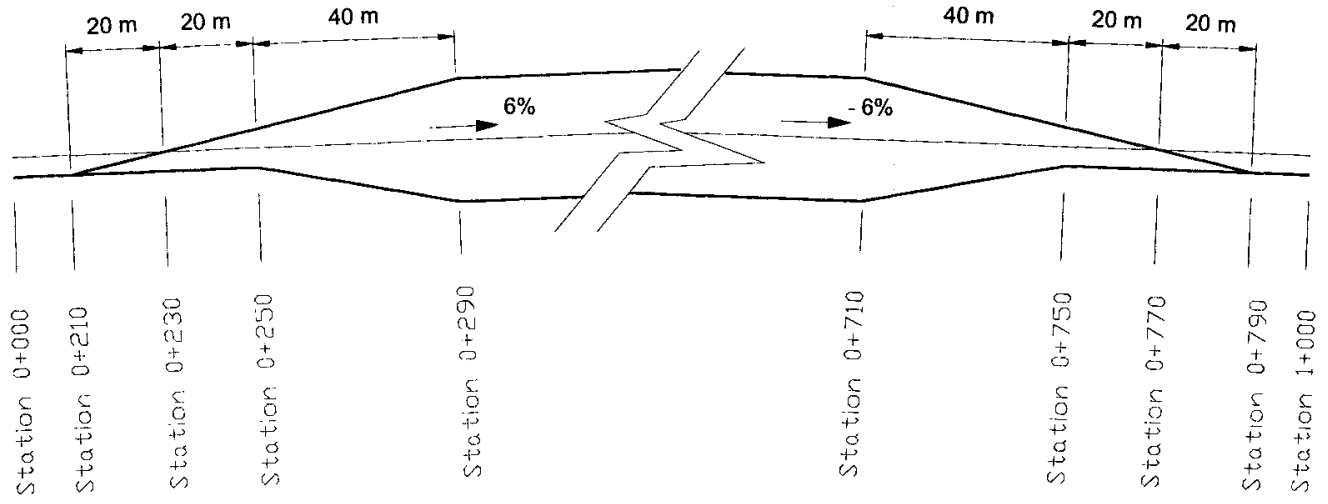

Figure 5-3: Assumed Cross-sections and Progress of Superelevation.
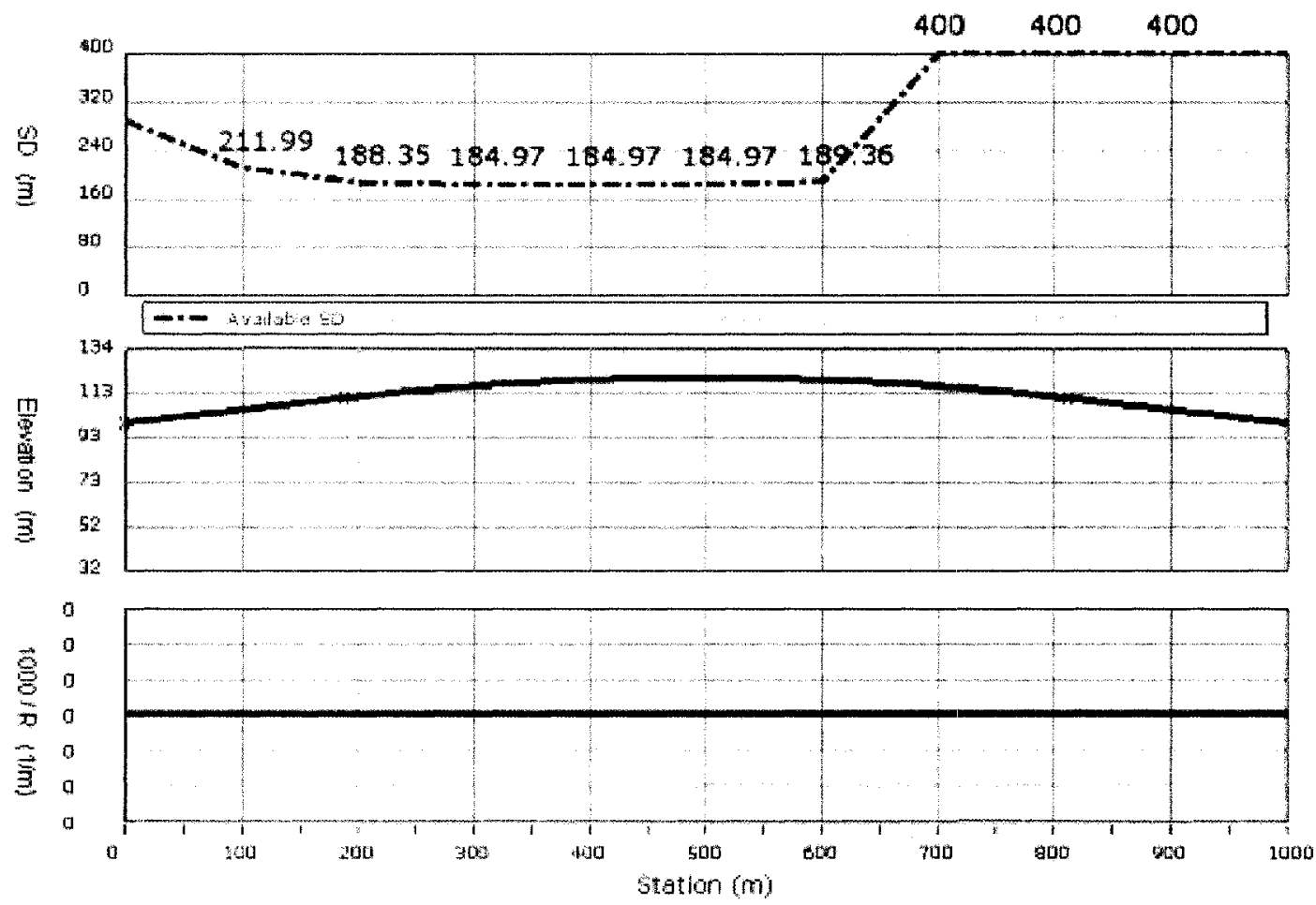

Figure 5-4: 2D-V AvSD Profile. 
The top part of this figure indicates that AvSD is lowest when both driver eye and object are located on the vertical curve. The figure also shows that a distance of $184.97 \mathrm{~m}$ was available for drivers on a substantial length of the road. This value is the exact result from the analytical approach (Equation 5-6) when values of $1.08 \mathrm{~m}, 0.6 \mathrm{~m}, 624 \mathrm{~m}$, and 12 $\%$ are used for $h_{1}, h_{2}, L_{\mathrm{c}}$, and $A$, respectively. Given that the values of the two simulation approaches coincide with the analytical formula, the 2D-V AvSD from SDES was considered suitable and reliable for further analysis.

\subsubsection{AvSD on Horizontal-Only Alignment}

Similar to $2 \mathrm{D}-\mathrm{V}$ AvSD, two approaches are also available to calculate $2 \mathrm{D}-\mathrm{H}$ AvSD. As shown in Figure 5-5, two profiles have been plotted by SDES. The first profile in the very top part is the one resulting from simulating the $2 \mathrm{D}$ analytical technique whereas the second profile is the one based on the $3 \mathrm{D}$ simulation of a horizontal-only curve with the vertical alignment being a flat segment. It is obvious that AvSD along the steady part of the first profile (i.e., $183.21 \mathrm{~m}$ ) is slightly higher than the corresponding value of the second profile (i.e., $183.15 \mathrm{~m}$ ). By substituting the related design values back in Equation 5-2 and Equation 5-3, it was found that the first profile provides almost the exact AvSD to the one resulting from the analytical technique (i.e. $183.20 \mathrm{~m}$ ). This was expected since the first profile is the simulation of the same analytical method. However, the second profile indicates that $3 \mathrm{D}$ AvSD is providing a value that is slightly higher by $0.06 \mathrm{~m}$. Although the difference is trivial in relative terms, explanation was still required. 


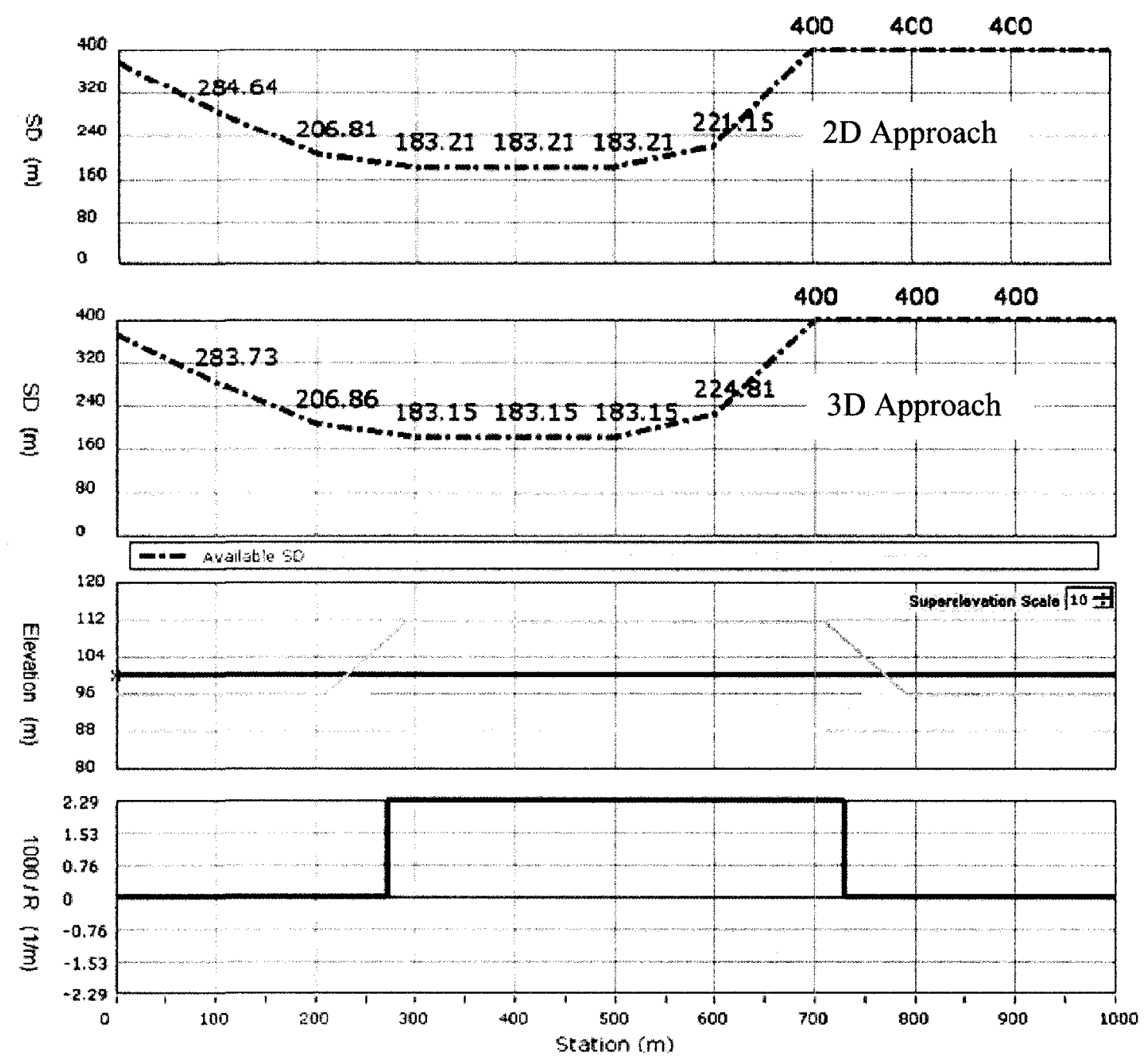

Figure 5-5: 2D versus 3D Approaches for AvSD on Flat Horizontal Curves.

The difference can be explained that, in case of lateral obstruction due to side slope on a horizontal curve, the analytical methodology assumes that the point of tangency between the sight line and the side slope cone is located exactly at the midpoint between the driver eye and the object (see Figure 5-6). However, this assumption is valid only when $h_{1}$ is equal to $h_{2}$. The point of tangency, however, starts to move to the left or right if $h_{1}$ is either higher or lower, respectively. 


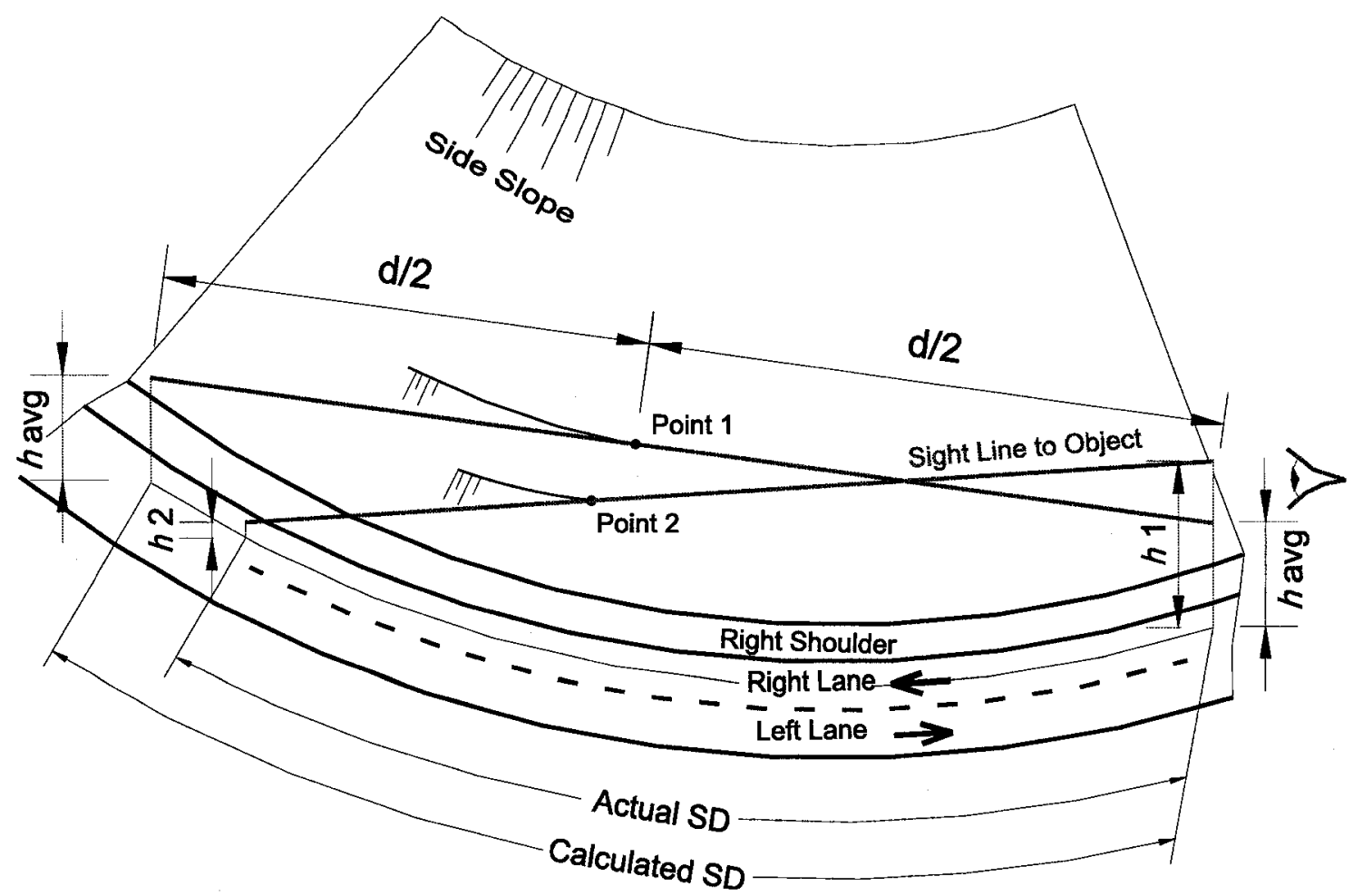

Figure 5-6: Difference between 2D and 3D AvSD on Horizontal-Only Curves.

Since $h_{1}$ is often higher than $h_{2}$, the point of tangency is expected to move on the cone surface toward the lower left side. This very slight shift could result in a little difference between the 2D-H and 3D AvSD on horizontal-only curves. The difference depends mainly on the side slope as it decreases with the decrease in the side slope and becomes zero for vertical obstructions. Having the results of the conducted checks, SDES was assumed to be reasonably ready for the rest of the study.

\subsection{Probabilistic versus Deterministic AvSD}

As currently applied in the design guides, the deterministic approach utilizes single values for both $h_{1}$ and $h_{2}$ as the two main parameters causing variations of AvSD. 
In TAC (1999), values of 1.05 and $0.38 \mathrm{~m}$ were said to be sufficient for $h_{1}$ and $h_{2}$, respectively. AASHTO (2004), however, recommends higher values of 1.08 and $0.6 \mathrm{~m}$ (see Table 5-1). On the other hand, the mean and standard deviation that are essential for the probabilistic approach were evaluated by Fambro (1997) as shown in Table 5-1.

Table 5-1: Summary of $h_{1}$ and $h_{2}$ (Deterministic and Probabilistic).

\begin{tabular}{ccccc}
\hline \multirow{2}{*}{ Heights } & \multicolumn{2}{c}{ Deterministic Approach } & \multicolumn{2}{c}{ Probabilistic Approach } \\
& TAC & AASHTO & mean & $\sigma$ \\
\hline$h_{1}(\mathrm{~m})$ & 1.05 & 1.08 & 1.149 & 0.055 \\
$h_{2}(\mathrm{~m})$ & 0.38 & 0.60 & 0.726 & 0.07 \\
\hline
\end{tabular}

In the following few sections, SDES will be run using different alignment configurations. Comparison will also be conducted between SDES results and those of the analytical technique, where possible.

\subsubsection{D-V AvSD: Simulation Method}

To show the difference between the deterministic and probabilistic design methods considering only the vertical alignment, SDES was run using 100,000 iterations with mean and standard deviation values as listed in Table 5-1. The values recommended by TAC (1999) and AASHTO (2004) were also used to perform the deterministic approach for comparison purposes. The top part of Figure 5-7 displays two fairly close profiles. The figure shows that the minimum AvSD values of 196.20 and $164.83 \mathrm{~m}$ were obtained on the middle part of the profiles for the mean probabilistic and TAC deterministic approaches, respectively. For more clarification, the 100,000 AvSD values at Station 400 were extracted and plotted using Matlab. As shown in Figure 5-8, AvSD distribution has a mean of $196.20 \mathrm{~m}$ and a standard deviation of $4.97 \mathrm{~m}$. 


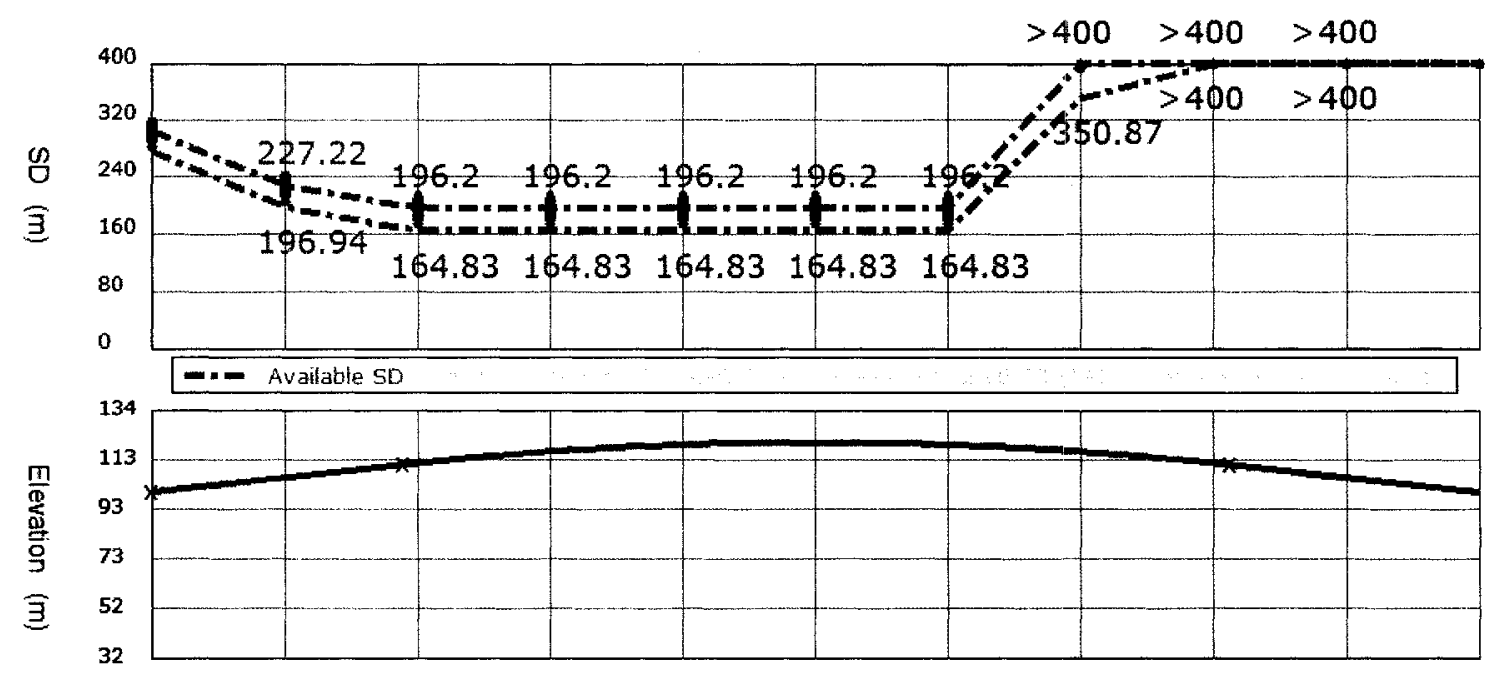

Figure 5-7: TAC 2D-V AvSD profile versus Probabilistic Approach.

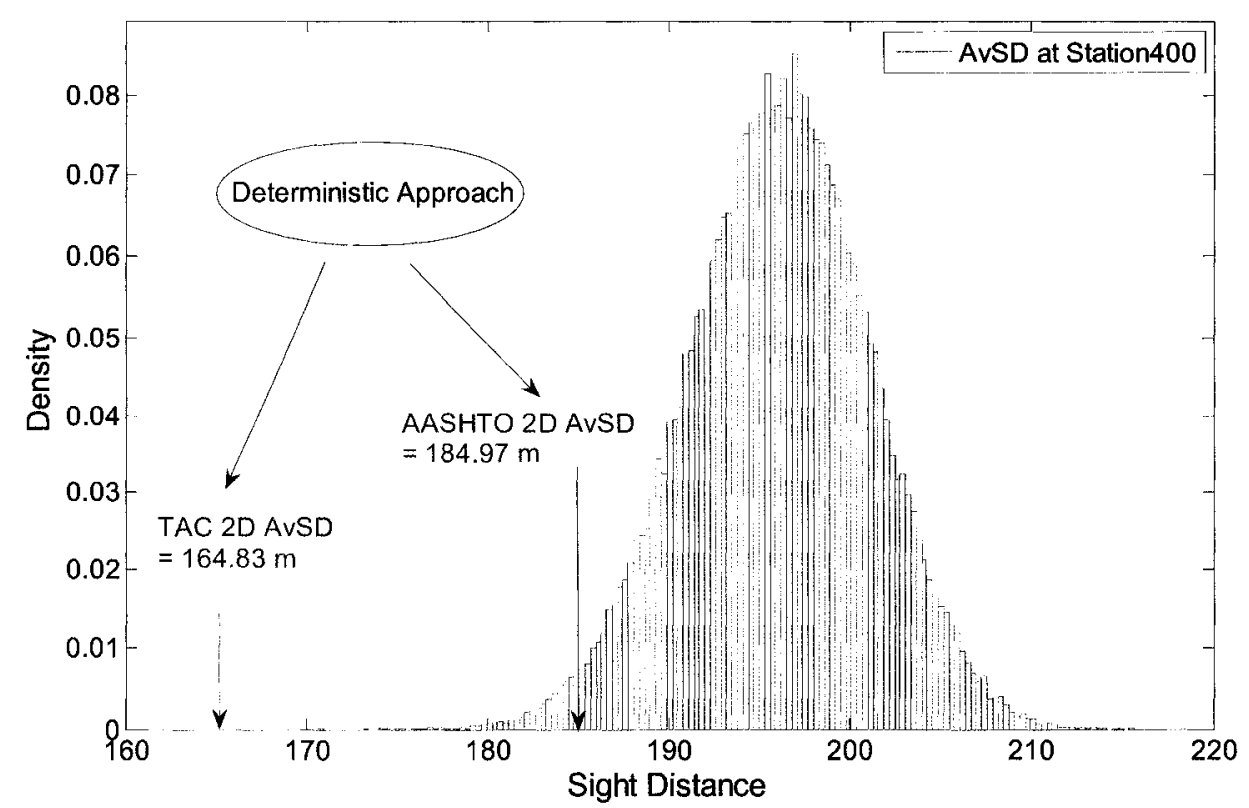

Figure 5-8: Deterministic versus Probabilistic 2D-V AvSD at Station 400. 
The figure also shows the two AvSD values resulting from the use of TAC and AASHTO recommended $h_{1}$ and $h_{2}$ values. Interestingly, the entire AvSD distribution is greater than the TAC AvSD of 164.83 m whereas, out of 100,000 trials, a total of 1480 cases $(1.48 \%$ ) were found to have AvSD less than the AASHTO AvSD of $184.97 \mathrm{~m}$. Assuming that the distributions of $h_{1}$ and $h_{2}$ are fairly representing real world values, the results would indicate the extreme conservative nature of both AASHTO and TAC by having a design percentile value of more than $98.5 \%$. Even though larger designs are always recommended, the simulation indicates that the use of curves shorter than those recommended by TAC and AASHTO may not be dramatically hazardous.

\subsubsection{D-V AvSD: Analytical Technique}

Using the FORM reliability method discussed in Section 3.1.3 and back to Equation 5-6 for 2D-V AvSD, one can describe its variation in terms of variations of both $h_{1}$ and $h_{2}$. The variance, as shown in Equation 5-7, includes a correlation factor between $h_{1}$ and $h_{2}$ as part of the equation. However, this term can be excluded with the logical independency between the two variables. It should also be emphasized that mean $2 \mathrm{D}-\mathrm{V}$ AvSD can be estimated by substituting mean values of $h_{1}$ and $h_{2}$ in Equation 5-6. Using values of 0.055 and $0.07 \mathrm{~m}$ for $\sigma_{\mathrm{h} 1}$ and $\sigma_{\mathrm{h} 2}$, respectively, a standard deviation of $4.94 \mathrm{~m}$ was resulted for 2D-V AvSD. In addition, using values of 1.149 and 0.726 for mean $h_{1}$ and $h_{2}$, respectively, was found yielding the same mean of $196.20 \mathrm{~m}$ as the simulation technique. The result indicates that only $0.03 \mathrm{~m}(0.6 \%)$ difference in the standard deviation exists between the simulation method and the analytical one. However, the 
applicability of the analytical technique is still limited to cases where only vertical curves are implemented.

$$
\begin{aligned}
\operatorname{var}(S)=\left(\frac{\partial S}{\partial h_{1}}\right)^{2}{\sigma_{h_{1}}}^{2}+\left(\frac{\partial S}{\partial h_{2}}\right)^{2} \sigma_{h_{2}}{ }^{2} \\
+2\left(\frac{\partial S}{\partial h_{1}}\right)\left(\frac{\partial S}{\partial h_{2}}\right) \operatorname{cov}\left[h_{1}, h_{2}\right]
\end{aligned}
$$

where;

$$
\begin{gathered}
\frac{\partial S}{\partial h_{1}}=5 \sqrt{\frac{2 L_{C}}{A h_{1}}} \\
\frac{\partial S}{\partial h_{2}}=5 \sqrt{\frac{2 L_{C}}{A h_{2}}}
\end{gathered}
$$

Equation 5-8

Equation 5-9

\subsubsection{D-H AvSD: Simulation Method}

Similar to the case of 2D-V AvSD, SDES was run again on horizontal-only curve with 437-m radius. As shown in Figure 5-9, two profiles are presented to express the probabilistic 2D-H AvSD along with TAC deterministic AvSD. Obviously the two profiles are fairly close having their minimum when both the driver eye and object are located on the horizontal curve. The figure indicates that a mean value of $185.06 \mathrm{~m}$ and a standard deviation of 0.85 for the probabilistic approach are expected on the mid part of the segment whereas a value of $180.51 \mathrm{~m}$ represents the TAC deterministic approach. In addition, SDES was run again using $h_{1}$ and $h_{2}$ recommended by AASHTO and AvSD profile indicated that a minimum AvSD of $183.21 \mathrm{~m}$ is expected on the mid part of the curve. 

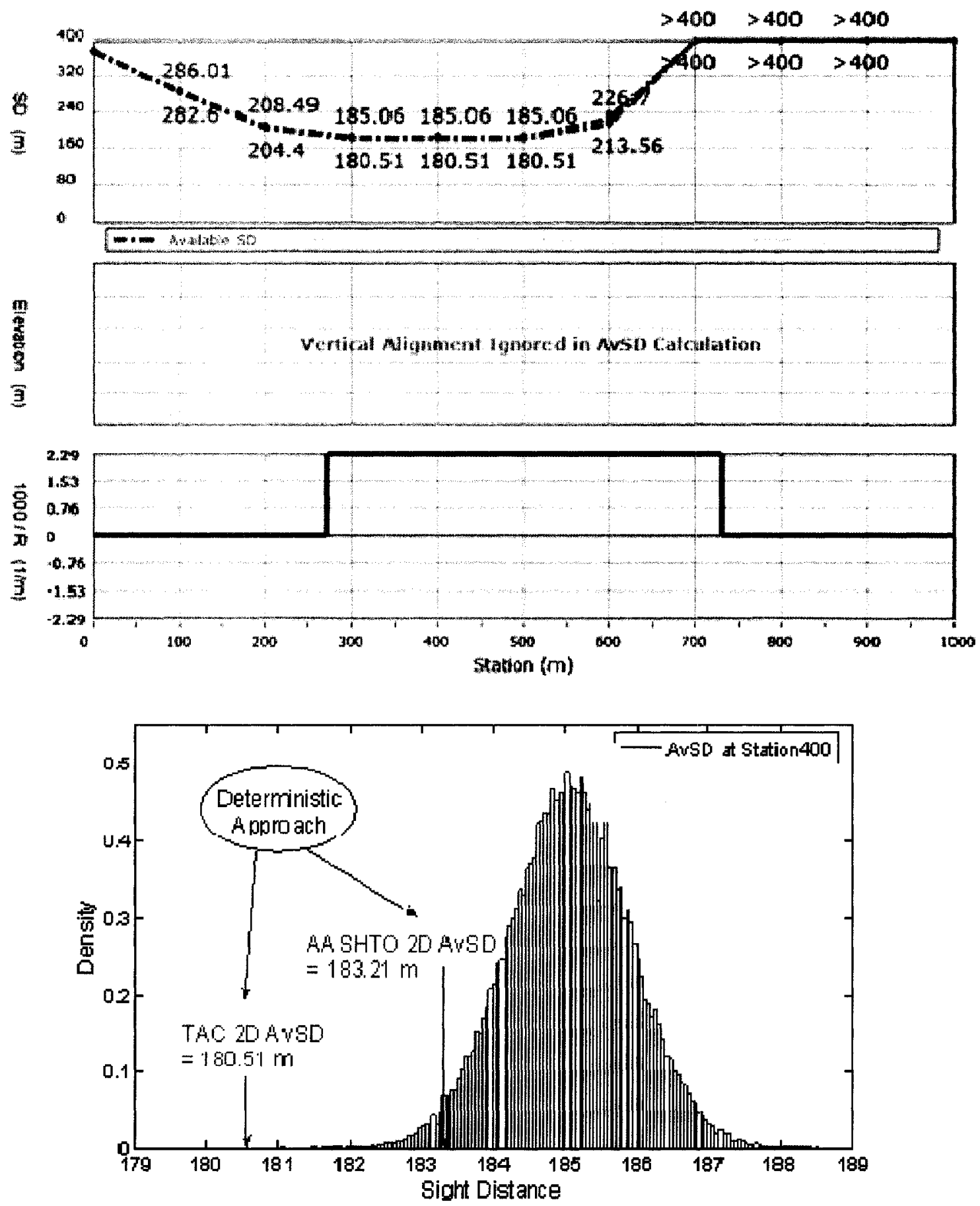

Figure 5-9: Profile and Distribution of 2D-H AvSD at Station 400. 
By plotting the distribution of AvSD at Station 400 as an example along with both TAC and AASHTO deterministic values, the results would re-stress on the conservative assumptions of current design guides. In more detail, only 1404 drivers out of 100,000 (1.4\%) were found demanding AvSD higher than the value recommended by AASHTO while all drivers would be satisfied using TAC criteria. This suggests that the current practice could be uneconomic besides its intuitive rigidity.

Furthermore, a quick comparison between the standard deviation of the $2 \mathrm{D}-\mathrm{V}$ $(4.97 \mathrm{~m})$ and $2 \mathrm{D}-\mathrm{H}(0.85 \mathrm{~m})$ distributions at Station 400 indicated that, as expected, the disparity is sensitive to the slope of the surface that obstructs the sight line. While vertical surface may eliminate the variation of AvSD to zero, variance may reach its maximum when a flat surface obstructs the sight line. For further evaluation of such sensitivity, the same alignment was used four times with different side slopes including $1 \mathrm{H}: 1 \mathrm{~V}, 2 \mathrm{H}: 1 \mathrm{~V}$, $3 \mathrm{H}: 1 \mathrm{~V}$, and $4 \mathrm{H}: 1 \mathrm{~V}$. The four AvSD distributions at Station 400 were plotted in the same graph as shown in Figure 5-10. As per the discussion above, the figure shows that the standard deviation may increase to more than three time when the side slope increase from $1 \mathrm{H}: 1 \mathrm{~V}$ to $4 \mathrm{H}: 1 \mathrm{~V}$.

\subsubsection{D-H AvSD: Analytical Technique}

Using the formulas presented before to describe the 2D-H AvSD (Equation 5-2 and Equation 5-3), the moments of its distribution can be simply formulated in terms of variations of $h_{1}$ and $h_{2}$. While, as mentioned before, the mean values of 2D-H AvSD may be predicted by using mean values of $h_{1}$ and $h_{2}$, the variance can be written by conducting few simple derivatives as shown in Equation 5-10 through Equation 5-13. 


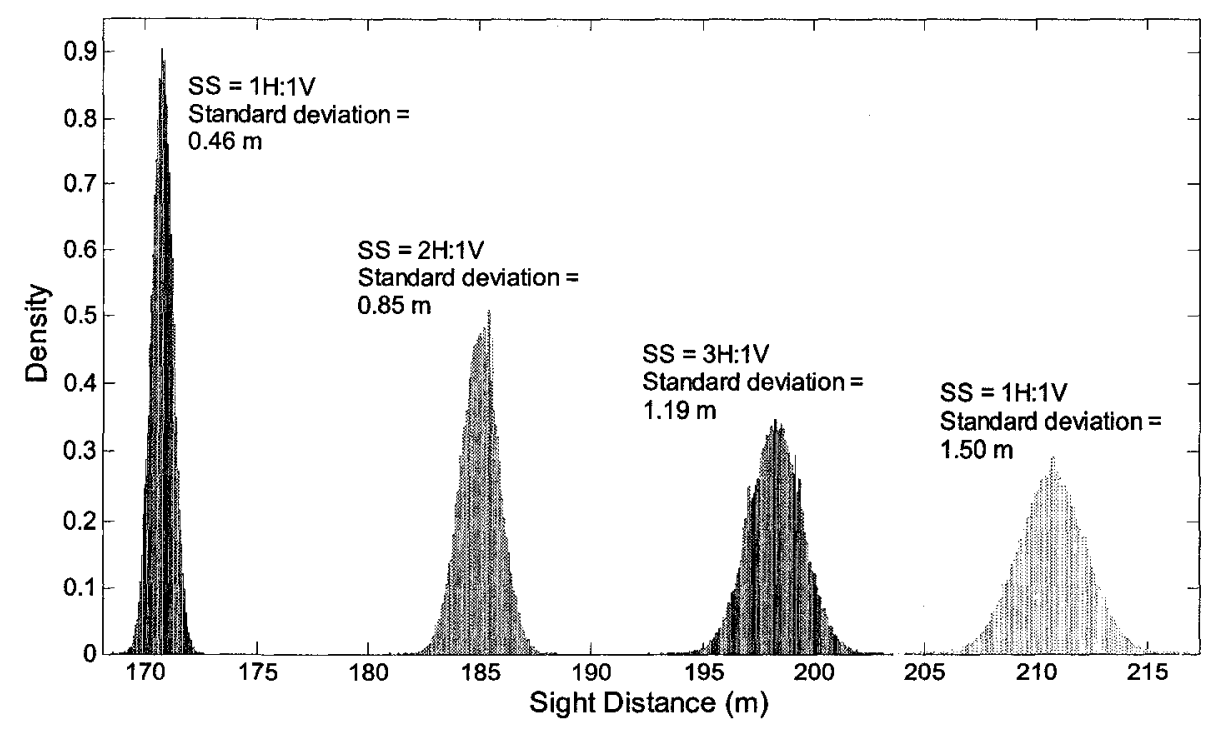

Figure 5-10: Influence of Side Slope on AvSD Variation.

The use of these equations with the numbers presented in the application example used earlier has resulted in a standard deviation of $0.84 \mathrm{~m}$. This value is almost the same as the one calculated by SDES $(0.85 \mathrm{~m})$ using the simulation technique. On one hand, this might confirm the suitability of the simulation approach to express the expected disparity of AvSD on horizontal curves. However, the equations are again limited to those cases where horizontal-only curves are constructed with longitudinal grades approaching zero.

$\operatorname{var}(S)=\left(\frac{\partial S}{\partial h_{1}}\right)^{2} \sigma_{h_{1}}{ }^{2}+\left(\frac{\partial S}{\partial h_{2}}\right)^{2}{\sigma_{h_{2}}}^{2}+2\left(\frac{\partial S}{\partial h_{1}}\right)\left(\frac{\partial S}{\partial h_{2}}\right) \operatorname{cov}\left[h_{1}, h_{2}\right] \quad$ Equation 5-10 where; $\quad \frac{\partial S}{\partial h_{1}}=\frac{\partial S}{\partial u} * \frac{\partial u}{\partial h_{1}}=\frac{1}{57.3}\left(\frac{S S}{\sqrt{1-u^{2}}}\right)$ Equation 5-11

$\frac{\partial S}{\partial h_{2}}=\frac{\partial S}{\partial u} * \frac{\partial u}{\partial h_{2}}=\frac{1}{57.3}\left(\frac{S S}{\sqrt{1-u^{2}}}\right)$ Equation 5-12 $u=1-\frac{H S O}{R}$ 


\subsubsection{D AvSD: Simulation Method}

Generally, the use of the 2D design approach is acceptable for either flat horizontal curves or straight vertical curves. However, the consideration of the $3 \mathrm{D}$ methodology becomes more demanded when complex combinations of horizontal and vertical curves are analyzed. In order to show the difference between the $2 \mathrm{D}$ and $3 \mathrm{D}$ methodologies in the example in hand, SDES was run and the two profiles of both probabilistic and TAC deterministic were plotted as shown in Figure 5-11. The AvSD distribution was further plotted in the same figure along with TAC and AASHTO deterministic values. It is obvious that the probabilistic distribution along with the deterministic values are all lower than those of the $2 \mathrm{D}$ approach because of the combination between the horizontal and crest vertical curves.

\subsection{D versus 3D Probabilistic AvSD}

The difference between the 2D and 3D AvSD has been well covered in the literature from a deterministic point of view. However, such difference is approached in this section from a probabilistic prospective. Generally, when a sag vertical curve overlaps with a horizontal curve, the curve would appear flatter and drivers would accordingly feel more comfortable compared to a horizontal-only curve (Smith and Lamm 1994). In addition, because the cone surface of the side slope shifts down, the road would provide more space and give more opportunity for road users to see farther. In such a case, the point of tangency between the sight line and the cone surface is expected to be higher and drivers would then have a greater chance to react safely when they attempt to stop for a certain reason. 

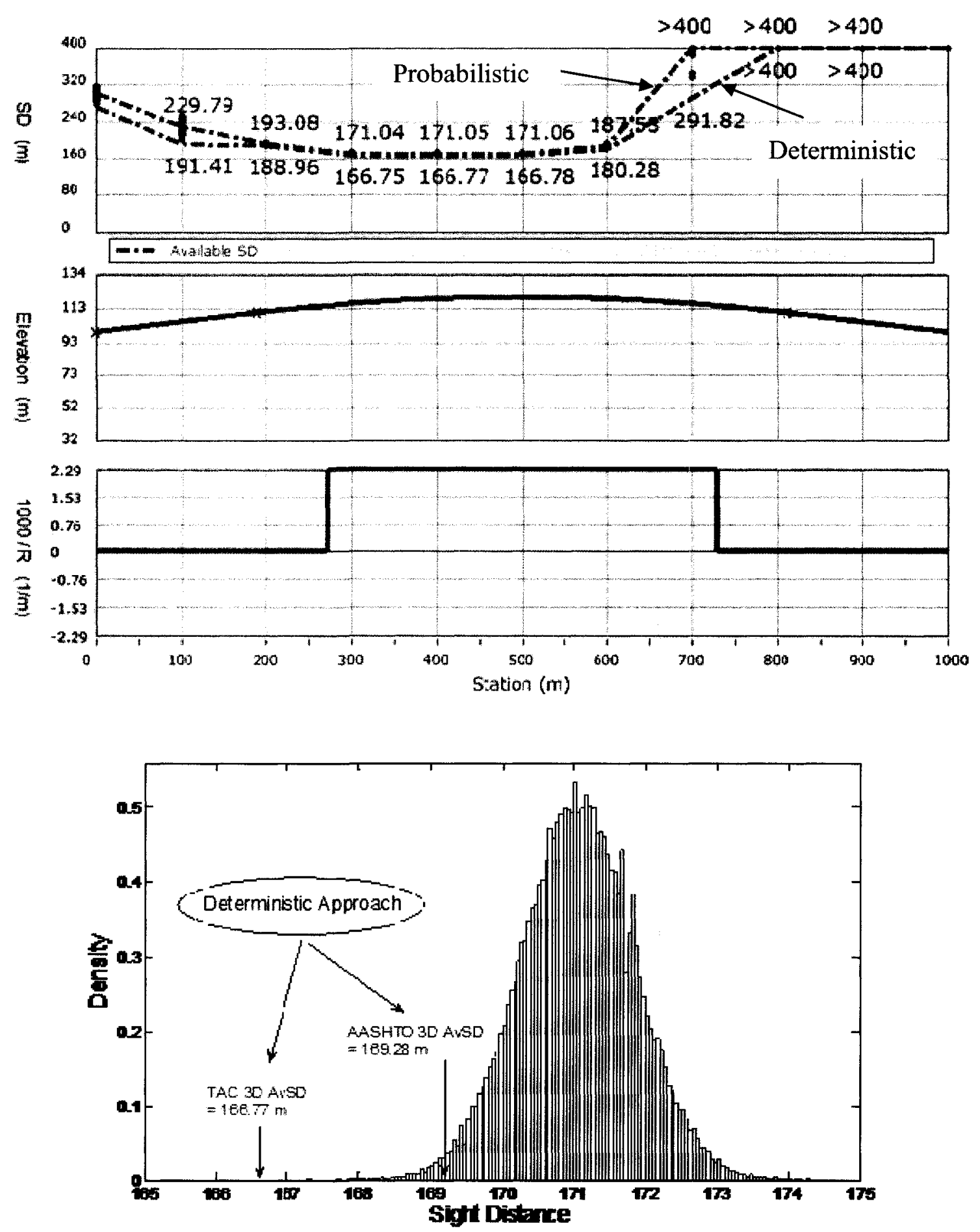

Figure 5-11: TAC Deterministic versus Probabilistic Approaches for 3D AvSD. 
On the other hand, the overlapping between a horizontal curve and a crest curve may cause the cone surface becomes relatively higher and the point of tangency would become closer to the road surface. This might cause the line of sight to be more restricted and drivers might accordingly adapt lower speeds on such curve. It can generally be said that AvSD would decrease with increasing the $K$ value of the vertical curve. The discussion above would explain in general the difference between the 2D and 3D and the influence that the vertical alignment may have on AvSD. However, the distributions of both 2D and 3D AvSD would also have different characteristics according to the road design.

For more clarification, Table 5-2 shows the difference between the $2 \mathrm{D}$ and $3 \mathrm{D}$ approaches at Station 400 in the example presented in this chapter. The table displays both absolute difference as well as the percent change with the 3D approach being the base case. It should be pointed out that the $2 \mathrm{D}$ values presented in this table correspond to the 2D-C AvSD that represent the more critical distribution of both $2 \mathrm{D}-\mathrm{H}$ and $2 \mathrm{D}-\mathrm{V}$ AvSD. The table also illustrates that the 3D AvSD distribution is $7 \%$ less than the $2 \mathrm{D}$ one and this significant reduction will be neglected in the design if only 2D AvSD is considered.

Table 5-2: Comparison between 2D and 3D design Approaches at Station 400.

\begin{tabular}{ccccc}
\hline & 2D Approach & 3D Approach & \multicolumn{2}{c}{ 2D versus 3D } \\
\cline { 4 - 5 } & $(\mathrm{m})$ & $(\mathrm{m})$ & Absolute $(\mathrm{m})$ & Percentage \\
\hline TAC & 180.51 & 166.77 & 13.74 & 7.6 \\
AASHTO & 183.21 & 169.28 & 13.93 & 7.6 \\
mean & 185.06 & 171.06 & 14.00 & 7.6 \\
$\sigma$ & 0.85 & 0.79 & 0.06 & 7.1 \\
\hline
\end{tabular}


Furthermore, the 2D and 3D AvSD distributions that were calculated at Station 400 were plotted together as shown in Figure 5-12. In this figure, the $3 \mathrm{D}$ distribution is not only indicating lower AvSD, but also showing a different standard deviation. This actually may create a challenge to predict the standard deviation given that no analytical approach is yet available in this matter. It should also be mentioned that the magnitude of the variation of the $3 \mathrm{D}$ AvSD is closer to the one of the $2 \mathrm{D}-\mathrm{H}$ since the line of sight where it intersects with the created road surface is probably passing through the side slope.

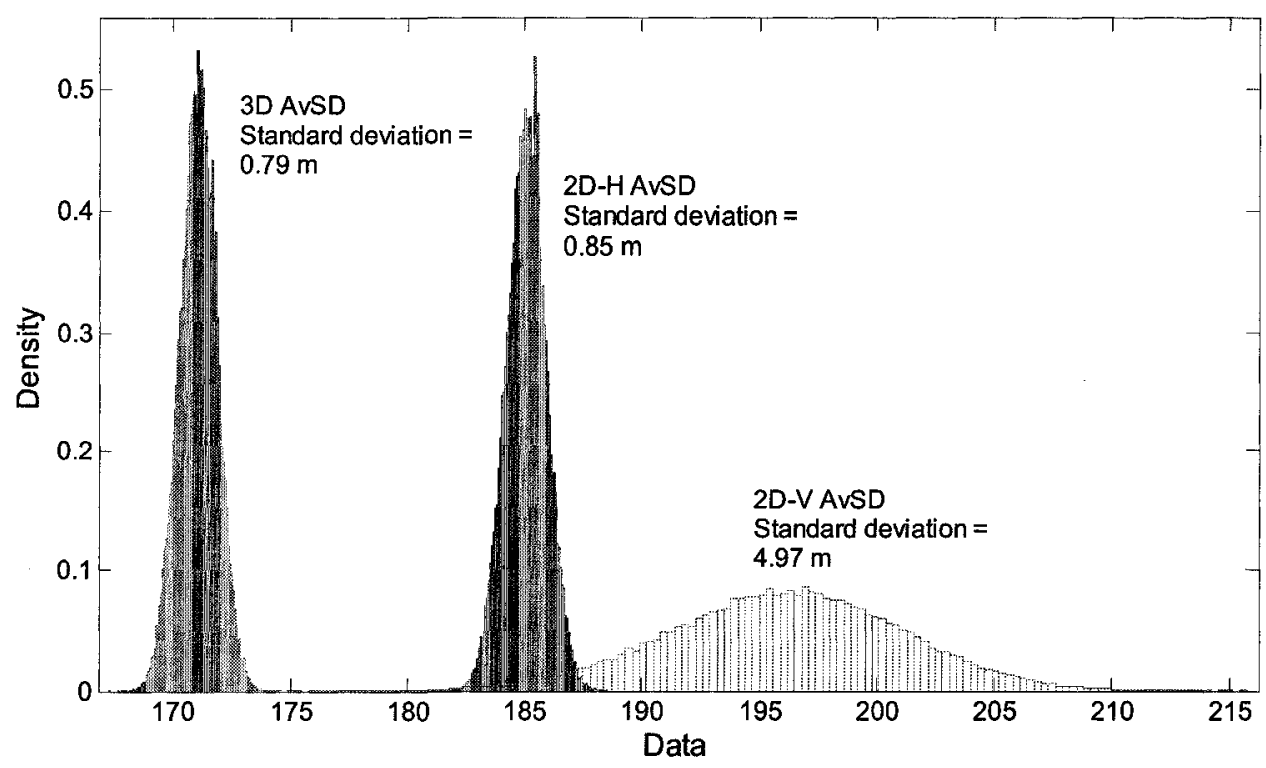

Figure 5-12: 2D versus 3D AvSD Distributions.

\subsection{Summary}

The discussion provided in this chapter shows the difference between the deterministic and probabilistic design approaches to identify the sight distance available 
to drivers at a given road section. The variation of this distance is caused primarily by variations in two parameters; namely driver eye and object heights. Using recent recommendations for these two parameters' variation, an application example showed that the expected variance of AvSD could be different from one case to another depending on the road configuration and analysis type (e.g., 2D and 3D). The numerical examples also indicated that both analytical and simulation reliability methods are almost identical in expressing the variance of AvSD. The analytical method, however, is applicable for only those cases where an isolated horizontal or vertical curve is implemented separately. In all analyzed cases, the deterministic approach was found very conservative using the recommendations listed in both TAC (1999) and AASHTO (2004). Furthermore, a comparison between the 2D and 3D distributions indicated that the $3 \mathrm{D}$ variance is hard to predict in a way that suggests the performance of the simulation reliability technique as the only available approach. 


\section{CHAPTER 6}

\section{HAZARd ASSessment AND CASE STUdy}

In this chapter, the probabilistic approach was used to assess the anticipated hazard due to insufficient sight distance along 3D alignments. Using this methodology, a hazard profile can be generated based on two RqSD and AvSD profiles, which can be generated as described in CHAPTER 4 and CHAPTER 5, respectively. The hazard profile should also help locate road sections with relatively higher risk levels than normal. In this chapter, Section 6.1 provides a general discussion of the probabilistic design approach. In Section 6.2 , the hazard evaluation methodology was applied on a real case study and findings were then highlighted. Finally, the interrelation between the probability of hazard and probability of collision is covered in Section 6.3 .

\subsection{Introduction}

In CHAPTER 4 and CHAPTER 5, the deterministic and probabilistic approaches were applied to evaluate both RqSD and AvSD, separately, in a way that highlighted the conservative nature of the current design practice. As an example, Figure 6-1 shows two RqSD and AvSD distributions that were extracted from previous runs in the last two chapters. The figure also shows two lines that approximate the typical location of the deterministic values near the end of both RqSD and AvSD distributions. 


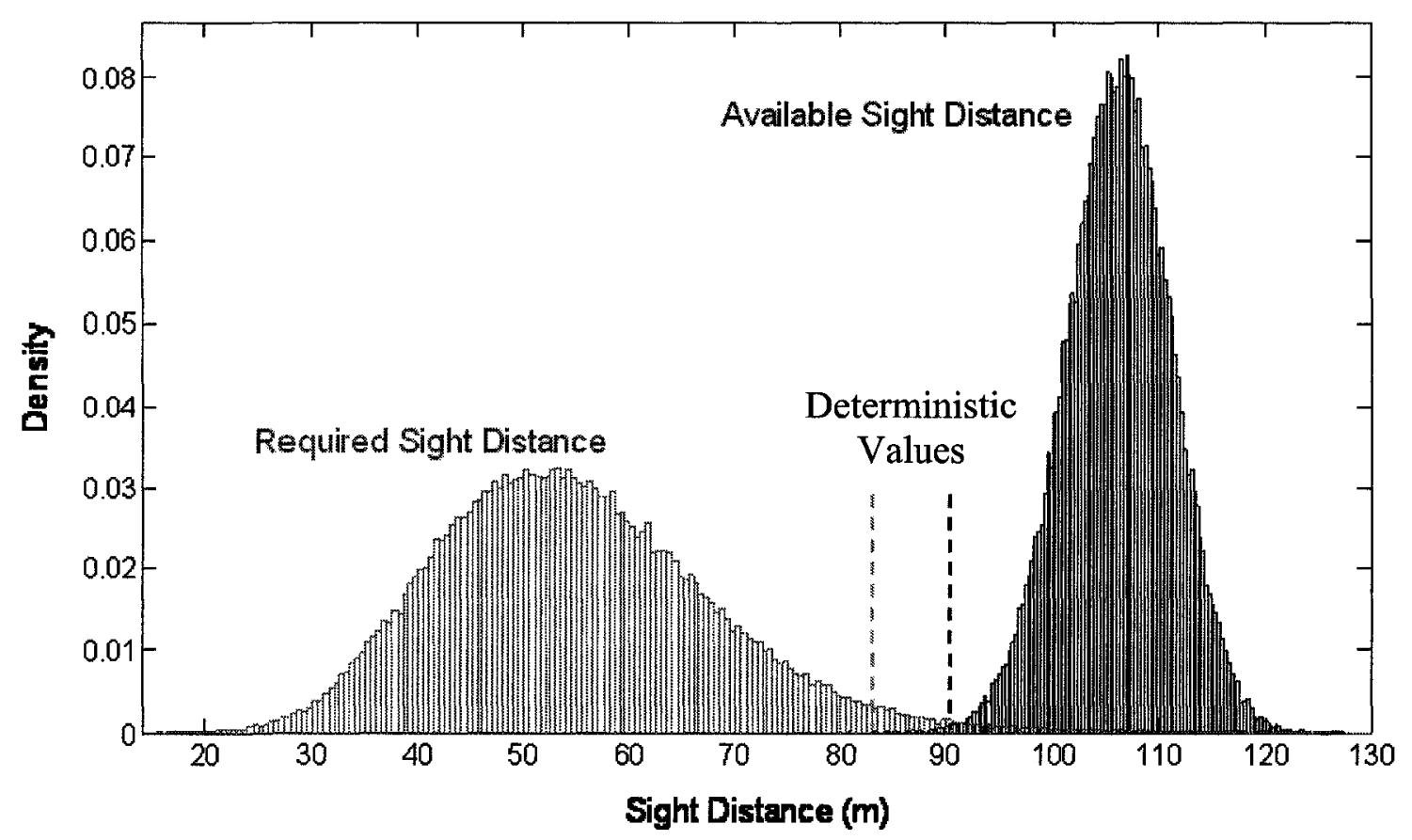

Figure 6-1: RqSD and AvSD Distributions.

On one hand, it was indicated in CHAPTER 4 that less than $1 \%$ of drives may require a higher sight distance than the deterministic value. Similarly in CHAPTER 5, the use of either horizontal or vertical road projections to calculate the deterministic AvSD, as the case in current design guides, would probably be sufficient such that almost all drivers can see even farther. The current design guides hence assume that a design is theoretically appropriate as long as the deterministic AvSD value is greater than the deterministic RqSD value. To a great extent, this is true since, at such design scenario, the two distributions are far enough from each other to provide a safe operating environment for the majority of drivers. However, with this implicit safety consideration, a question would arise about the sensitivity of hazard to the margin between the two distributions. Answering this question may also allow more understanding of the 
expected consequences and anticipated hazard if, for some reason, the deterministic AvSD could not meet the deterministic RqSD. In such case, the two distributions may overlap resulting in high hazard levels. This latter scenario was reported in TAC (1999) to be allowed as exceptional "in rare cases where the cost of such provision and reduction in normal operation is feasible." However, no hazard assessment is yet possible with the obvious inability of the current deterministic approach to handle such rare cases.

\subsection{Case Study}

Using the methodology of hazard evaluation presented earlier in CHAPTER 3, $\mathrm{POH}$ can be used to describe the percentage of drivers who may suffer insufficient sight distance. To show the applicability of this approach, real data were used in this section using an 8-km two-lane segment of Highway 61 located in Ontario. The following few subsections will provide a description of the road segment, speed modelling, attempts to determine the friction distribution, and finally the simulation results showing the AvSD, $\mathrm{RqSD}$, and $\mathrm{POH}$ profiles.

\subsubsection{Road Segment Description}

The road segment used in this section is an $8-\mathrm{km}$ stretch (Station $10+000$ to $18+000$ ) of Highway 61 located in the township of Crooks, Ontario. The segment is a two-lane stretch located between the Canada-US borders and the City of Thunder Bay. In addition, a passing lane is added along $1.81 \mathrm{~km}$ stretch (station $10+740$ to station $12+550$ ) to the right direction and $1.58 \mathrm{~km}$ (station $16+420$ to $17+000$ ) to the left 
direction (Hassan 1996). Moreover, the design speed of the road is $110 \mathrm{~km} / \mathrm{h}$ whereas the speed limit is $90 \mathrm{~km} / \mathrm{h}$.

As shown in Figure 6-2, the horizontal alignment includes a variety of tangents, curves, and transition curves. In more detail, the horizontal alignment consists of 46 segments including 12 straight segments, 12 simple curves, and 22 transition curves (see Table 6-1). The 12 straight segments had a range of length from 68.58 to $890.10 \mathrm{~m}$. In addition, the 12 curves ( 6 curves turning right and 6 curves turning left) had a range of radius from 450 to $1800 \mathrm{~m}$ and a range of length from 51.68 to $556.44 \mathrm{~m}$. Furthermore, the range of length of the 22 transition curves was found from 28.14 to $133.24 \mathrm{~m}$. On the other hand, Table 6-2 presents a description of the vertical alignment that consists of 31 segments including 6 tangents, 5 curves, and 20 spline-grade segments. The 6 tangents had lengths ranging from 16 to $555 \mathrm{~m}$ while the maximum longitudinal grade is $6.57 \%$. Moreover, the 5 vertical curves had a range of length from 26 to $312 \mathrm{~m}$.

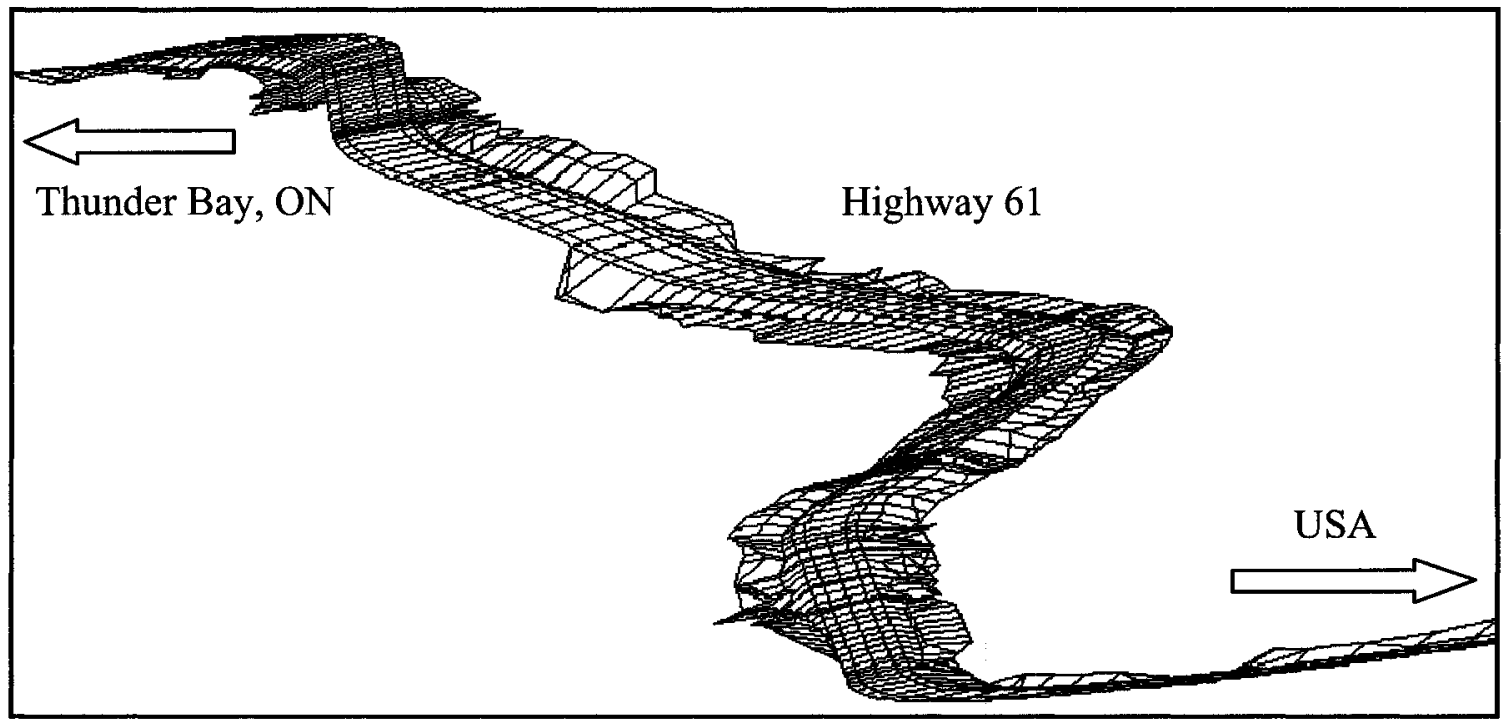

Figure 6-2: Three-Dimensional Schematic of Highway 61 Segment Used in the Analysis. 
Table 6-1: Horizontal Alignment of Highway 61.

\begin{tabular}{|c|c|c|c|c|c|c|}
\hline Segment \# & 1st station & Type & Direction & Length $(\mathrm{m})$ & $R_{1}(\mathrm{~m})$ & $R_{2}(\mathrm{~m})$ \\
\hline 1 & 10000.00 & Straight & direct & 356.78 & $\operatorname{lnf}$ & $\operatorname{lnf}$ \\
\hline 2 & 10356.78 & Spiral & left & 54.00 & Inf & 800 \\
\hline 3 & 10410.78 & Curve & left & 131.09 & 800 & 800 \\
\hline 4 & 10541.87 & Spiral & left & 54.00 & 800 & Inf \\
\hline 5 & 10595.87 & Straight & direct & 215.34 & Inf & $\operatorname{lnf}$ \\
\hline 6 & 10811.21 & Spiral & left & 62.78 & Inf & 575 \\
\hline 7 & 10874.00 & Curve & left & 167.62 & 575 & 575 \\
\hline 8 & 11041.62 & Spiral & left & 62.78 & 575 & $\operatorname{lnf}$ \\
\hline 9 & 11104.40 & Straight & direct & 231.31 & Inf & Inf \\
\hline 10 & 11335.71 & Spiral & left & 54.91 & Inf & 590 \\
\hline 11 & 11390.62 & Curve & left & 180.33 & 590 & 590 \\
\hline 12 & 11570.95 & Spiral & left & 54.92 & 590 & Inf \\
\hline 13 & 11625.86 & Straight & direct & 890.10 & $\operatorname{lnf}$ & Inf \\
\hline 14 & 12515.96 & Spiral & right & 72.00 & $\operatorname{lnf}$ & 450 \\
\hline 15 & 12587.96 & Curve & right & 86.58 & 450 & 450 \\
\hline 16 & 12674.54 & Spiral & right & 72.00 & 450 & $\operatorname{Inf}$ \\
\hline 17 & 12746.54 & Straight & direct & 226.10 & Inf & $\operatorname{lnf}$ \\
\hline 18 & 12972.65 & Spiral & left & 57.86 & $\operatorname{lnf}$ & 560 \\
\hline 19 & 13030.50 & Curve & left & 167.16 & 560 & 560 \\
\hline 20 & 13197.66 & Spiral & left & 57.86 & 560 & $\ln f$ \\
\hline 21 & 13255.52 & Spiral & right & 133.24 & $\operatorname{lnf}$ & 560 \\
\hline 22 & 13388.76 & Curve & right & 78.65 & 560 & 560 \\
\hline 23 & 13467.41 & Spiral & right & 71.43 & 560 & $\operatorname{lnf}$ \\
\hline 24 & 13538.84 & Straight & direct & 292.33 & Inf & Inf \\
\hline 25 & 13831.17 & Curve & right & 51.68 & 500 & 500 \\
\hline 26 & 13882.85 & Straight & direct & 588.64 & Inf & $\operatorname{lnf}$ \\
\hline 27 & 14471.48 & Spiral & right & 36.57 & Inf & 700 \\
\hline 28 & 14508.06 & Curve & right & 556.44 & 700 & 700 \\
\hline 29 & 15064.50 & Spiral & right & 36.57 & 700 & $\ln f$ \\
\hline 30 & 15101.07 & Straight & direct & 464.32 & $\operatorname{lnf}$ & $\operatorname{Inf}$ \\
\hline 31 & 15565.39 & Spiral & right & 62.24 & $\operatorname{lnf}$ & 850 \\
\hline 32 & 15627.63 & Curve & right & 112.08 & 850 & 850 \\
\hline 33 & 15739.70 & Spiral & right & 62.24 & 850 & Inf \\
\hline 34 & 15801.94 & Straight & direct & 68.58 & $\operatorname{lnf}$ & Inf \\
\hline 35 & 15870.52 & Spiral & left & 28.44 & $\operatorname{lnf}$ & 900 \\
\hline 36 & 15898.97 & Curve & left & 267.52 & 900 & 900 \\
\hline 37 & 16166.49 & Spiral & left & 28.14 & 900 & $\operatorname{lnf}$ \\
\hline 38 & 16194.63 & Straight & direct & 733.93 & Inf & Inf \\
\hline 39 & 16928.56 & Spiral & right & 32.00 & $\operatorname{lnf}$ & 1800 \\
\hline 40 & 16960.56 & Curve & right & 208.59 & 1800 & 1800 \\
\hline 41 & 17169.15 & Spiral & right & 32.00 & 1800 & $\operatorname{lnf}$ \\
\hline 42 & 17201.15 & Straight & direct & 326.24 & Inf & $\operatorname{lnf}$ \\
\hline 43 & 17527.39 & Spiral & left & 42.32 & $\operatorname{lnf}$ & 1250 \\
\hline 44 & 17569.71 & Curve & left & 124.21 & 1250 & 1250 \\
\hline 45 & 17693.93 & Spiral & left & 42.32 & 1250 & Inf \\
\hline 46 & 17736.25 & Straight & direct & 263.75 & Inf & Inf \\
\hline
\end{tabular}

Note: $R=$ "Inf" indicates a road tangent. 
Table 6-2: Vertical Alignment of Highway 61.

\begin{tabular}{|c|c|c|c|c|c|c|}
\hline Segment & 1st station & Type & $\begin{array}{l}\text { Length } \\
(\mathrm{m})\end{array}$ & $\begin{array}{c}\text { 1st grade } \\
(\%)\end{array}$ & $\begin{array}{c}\text { 2nd grade } \\
(\%)\end{array}$ & $\begin{array}{l}\text { No. of } \\
\text { points }\end{array}$ \\
\hline 1 & 10000.00 & Spline curve & 200.00 & -- & -- & 3 \\
\hline 2 & 10200.00 & Spline curve & 210.78 & -- & -- & 7 \\
\hline 3 & 10410.78 & Spline curve & 244.22 & -- & -- & 8 \\
\hline 4 & 10655.00 & Spline curve & 145.00 & -- & -- & 5 \\
\hline 5 & 10800.00 & Straight & 16.00 & 0.198 & 0.198 & -- \\
\hline 6 & 10816.00 & Curve & 286.00 & 0.198 & 6.565 & -- \\
\hline 7 & 11102.00 & Straight & 18.00 & 6.565 & 6.565 & -- \\
\hline 8 & 11120.00 & Spline curve & 555.00 & -- & -- & 35 \\
\hline 9 & 11675.00 & Spline curve & 325.00 & -- & -- & 15 \\
\hline 10 & 12000.00 & Spline curve & 550.00 & -- & - & 25 \\
\hline 11 & 12550.00 & Spline curve & 350.00 & -- & -- & 26 \\
\hline 12 & 12900.00 & Straight & 64.00 & -1.278 & -1.278 & -- \\
\hline 13 & 12964.00 & Curve & 312.00 & -1.278 & -5.744 & -- \\
\hline 14 & 13276.00 & Straight & 44.00 & -5.744 & -5.744 & -- \\
\hline 15 & 13320.00 & Curve & 262.00 & -5.744 & -1.373 & -- \\
\hline 16 & 13582.00 & Straight & 18.00 & -1.373 & -1.373 & -- \\
\hline 17 & 13600.00 & Spline curve & 265.00 & -- & -- & 22 \\
\hline 18 & 13865.00 & Spline curve & 135.00 & -- & -- & 16 \\
\hline 19 & 14000.00 & Spline curve & 300.00 & -- & -- & 15 \\
\hline 20 & 14300.00 & Spline curve & 350.00 & -- & -- & 15 \\
\hline 21 & 14650.00 & Spline curve & 350.00 & -- & -- & 22 \\
\hline 22 & 15000.00 & Spline curve & 375.00 & -- & - & 17 \\
\hline 23 & 15375.00 & Spline curve & 464.00 & -- & -- & 35 \\
\hline 24 & 15839.00 & Spline curve & 261.00 & -- & -- & 28 \\
\hline 25 & 16100.00 & Spline curve & 400.00 & -- & -- & 23 \\
\hline 26 & 16500.00 & Curve & 26.00 & 0 & 1.184 & -- \\
\hline 27 & 16526.00 & Curve & 200.00 & 1.184 & 5.644 & -- \\
\hline 28 & 16726.00 & Straight & 24.00 & 5.644 & 5.644 & -- \\
\hline 29 & 16750.00 & Spline curve & 400.00 & -- & -- & 16 \\
\hline 30 & 17150.00 & Spline curve & 350.00 & -- & -- & 15 \\
\hline 31 & 17500.00 & Spline curve & 500.00 & -- & -- & 24 \\
\hline
\end{tabular}

For the 20 spline-grade segments, the number of points with known stations and elevations had a minimum of 3 and a maximum of 35 covering a range of length from 
135 up to $555 \mathrm{~m}$. It should be mentioned, however, that the total length of these splinegrade segments is $6730 \mathrm{~m}$, which indicates that the vertical alignment is defined mainly by spline-grades. Furthermore, a total of 255 cross-sections were available with information such as pavement width, superelevation, shoulder width and slope, and cut and fill side slopes. The road has mainly a $3.75-\mathrm{m}$ lane width, $2 \%$ normal crown slope, up to $5.9 \%$ superelevation, 1.5 to $2.5 \mathrm{~m}$ shoulder width, and around $6 \%$ shoulder slope.

\subsubsection{Speed Estimation on Two-Lane Roadways}

The speed distribution on Highway 61 was estimated using speed data that were collected in another study by Misaghi and Hassan (2005). These speed data were collected on a total of 20 curves located on four other two-lane roads in Ontario including King's Highway 41, Secondary Highways 31 and 43, and Country road 12. As shown in Table 6-3, the curves had various characteristics with a curve radius $(R)$ ranging from 317.52 to $873.19 \mathrm{~m}$ and curve length $(L)$ ranging from 100.14 to $1001.89 \mathrm{~m}$. The locations of these curves were also selected to be far enough from signalized and stopcontrolled intersections. In addition, the curves had low traffic volumes such that the collected speeds reflect a free flow condition, as possible. For each curve, speeds were collected during 24 hours on 3 or 5 points using pneumatic traffic counters/classifiers. The five points were placed at the approach and departure tangents along with the beginning, middle, and end of each curve. Furthermore, on each of these points, speeds were collected for both road directions. 
Table 6-3: Curve and Speed Database Used in Estimating Speed Distribution.

\begin{tabular}{cccccc}
\hline \multirow{2}{*}{ Curve \# } & Highway & \multirow{2}{*}{$R(\mathrm{~m})$} & $L(\mathrm{~m})$ & \multicolumn{2}{c}{ Speed $(\mathrm{km} / \mathrm{h})$} \\
\cline { 5 - 6 } & & 388.00 & 527.49 & 86.15 & 8.69 \\
2 & 31 & 583.00 & 377.03 & 87.66 & 8.62 \\
3 & 31 & 873.19 & 473.96 & 92.31 & 9.24 \\
4 & 31 & 873.19 & 464.19 & 96.46 & 12.12 \\
5 & 31 & 873.19 & 604.65 & 89.52 & 9.91 \\
6 & 43 & 388.00 & 203.99 & 82.08 & 13.36 \\
7 & 43 & 582.12 & 101.60 & 86.34 & 9.82 \\
8 & 43 & 582.12 & 110.91 & 86.75 & 11.34 \\
9 & 43 & 698.55 & 352.96 & 88.42 & 11.37 \\
10 & 12 & 436.59 & 233.04 & 89.79 & 10.89 \\
11 & 12 & 317.52 & 443.34 & 86.66 & 10.89 \\
12 & 12 & 317.52 & 445.47 & 85.52 & 10.38 \\
13 & 12 & 582.13 & 111.20 & 83.80 & 13.82 \\
14 & 41 & 388.08 & 159.45 & 90.95 & 12.71 \\
15 & 41 & 436.59 & 100.14 & 94.96 & 12.97 \\
16 & 41 & 436.59 & 204.66 & 88.21 & 9.60 \\
17 & 41 & 349.00 & 472.38 & 86.08 & 9.06 \\
18 & 31 & 367.66 & 579.98 & 85.84 & 13.08 \\
19 & 12 & 537.35 & 836.89 & 91.49 & 8.93 \\
20 & 41 & 635.05 & 1001.89 & 91.52 & 10.25 \\
\hline
\end{tabular}

The collected speeds were then filtered to exclude nighttime and non-free-flow traffic data identified by the time gap between successive vehicles. The data were further categorized into three types including passenger car (PC), light truck (LT), and heavy truck (HT) using collected vehicle information such as wheelbase, number of axles, axle spacing. However, only PC and LT were included in the analysis as per previous analysis and recommendations reported by Misaghi and Hassan (2005). Furthermore, only vehicles that were observed traversing all data collection points located on each curve were isolated and their speeds were subsequently used in the analysis. The reason of that 
was to exclude the vehicles that entered or left the road between the first and last of the five points of each curve. Filtering those vehicles was done using a Microsoft Excel's Macro that recognized a vehicle by its characteristics and tracked it on the entire curve.

\subsection{2.a Speed on curve}

After the filtration of the speed data, the mean (V50) and standard deviation ( $\sigma$ ) observed on the mid-point of each curve, for only tracked vehicles, were considered (see back in Table 6-3). Both V50 and $\sigma$ presented in this table were calculated for the two road directions. The V50 and $R$ columns were then imported to the Statistical Package for Social Science (SPSS) for analysis. Some transformations were further prepared including $\operatorname{Ln}(R), R^{2}, R^{3}, 1 / R$, and $1 / R^{2}$. These variables were used individually as independent variables seeking the best model of the V50 as a dependant variable.

In all modelling attempts, two curves (Curve \# 13 and 15) were described as outliers with the relatively highest standardized residuals generated by SPSS. After the removal of these two outliers, the trend of the other 18 points indicated an increase in V50 with the increase of $R$, as shown graphically in Figure 6-3. Using the linear regression option available in SPSS, the linear relationship presented in Table 6-4 was found significant at $5 \%$ level of significance. The SPSS output also indicated that the intercept and the coefficient of the variable $R$ in this equation were both significant at $5 \%$ level of significance; even though the adjusted coefficient of determination $\left(R^{2}\right)$ of 0.41 was low. It should be also mentioned that no improvement in $R^{2}$ was observed when other transformed variables were used. 
Table 6-4: Model for the Mean Speed on Horizontal Curves.

\begin{tabular}{cccc}
\hline$V 50=0.0114 R+82.33$ & \multicolumn{3}{c}{$\ldots\left(\right.$ Adjusted $R^{2}=0.41 ;$ Degree of Freedom $\left.=17\right)$} \\
\hline Coefficient & Estimate & $t$ value & Significance \\
\hline Constant & 82.33 & 45.36 & $<0.001$ \\
$R$ & 0.0114 & 3.56 & 0.003 \\
\hline
\end{tabular}

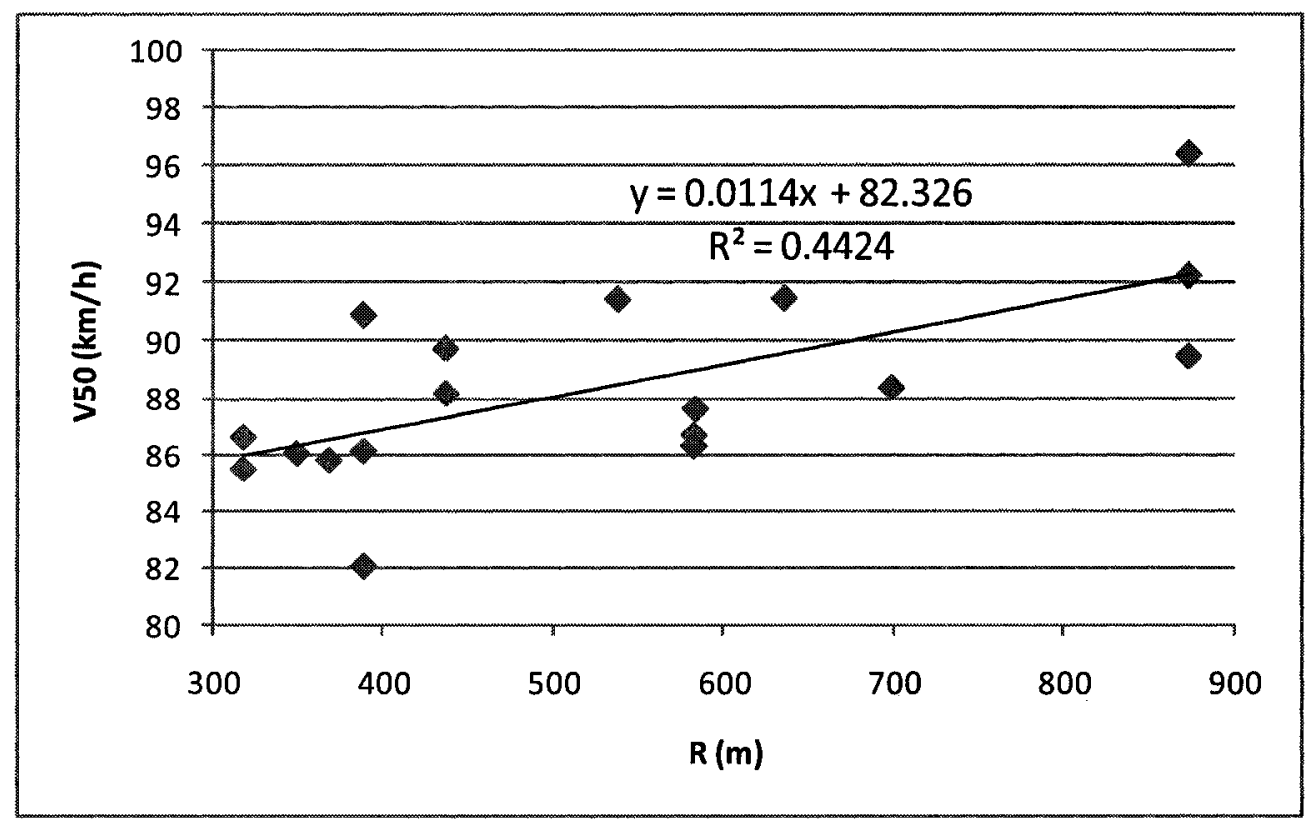

Figure 6-3: Curve Radius versus Mean Speed on Curves' Mid-Points.

On the other side, the scatter plot presented in Figure 6-4 shows the standard deviations of the speeds collected on the mid-points of the 20 curves. Similar to the case of V50, different models were tried using SPSS. However, no significant models were found and hence the average value of $10.85 \mathrm{~km} / \mathrm{h}$ was assumed as a best estimate of $\sigma$. 


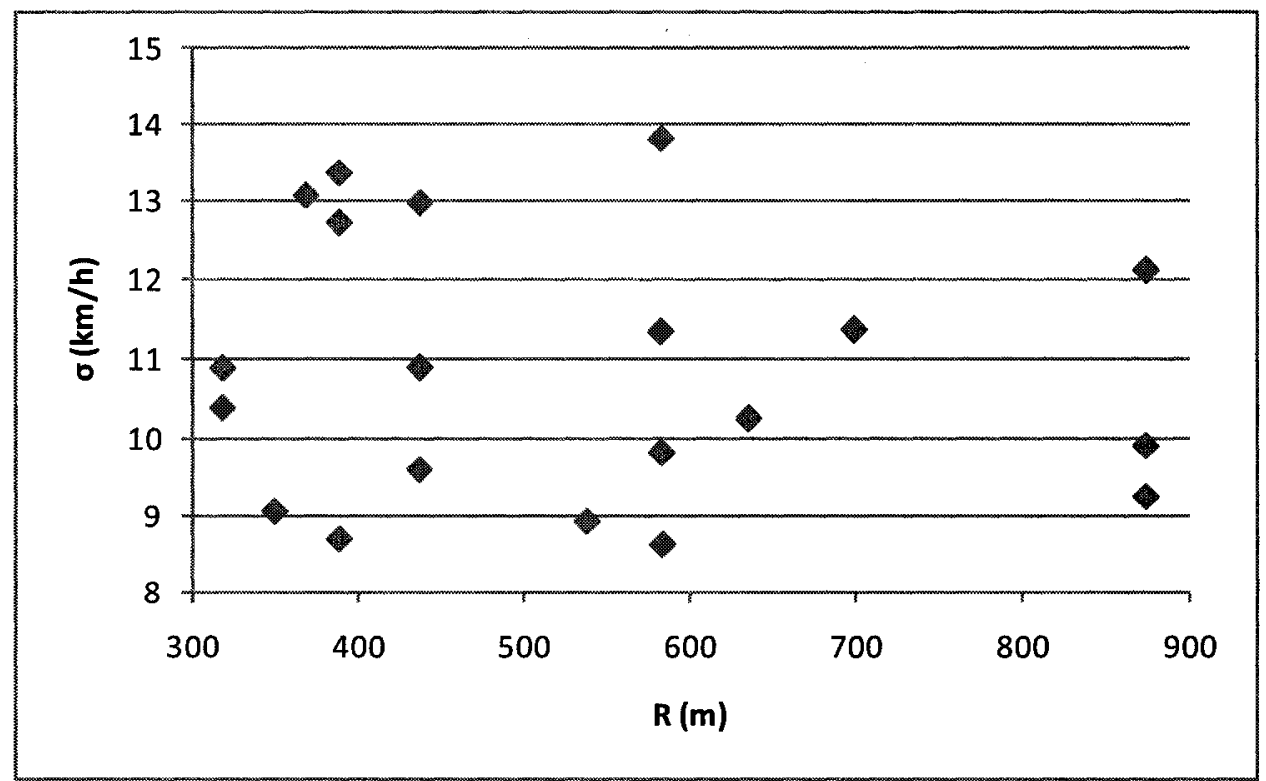

Figure 6-4: Curve Radius versus Standard Deviation of Speed on Curves' Mid-Points.

\subsection{2.b Speed on tangent}

As mentioned before, speeds were collected on three or five points on each of the 20 curves, among which the first and last points were located at the two tangents before and after each curve. Although data were collected for the two road directions, only those of approach tangents were considered. This is because a speed on a tangent would normally be influenced by the design of the curve ahead of the driver. Having 20 curves with two approach tangents for each curve, a total of 40 points can be considered for modelling. However, only 39 points were used since the information of one tangent was not available. Table 6-5 shows the radius and the length $\left(L_{\mathrm{T}}\right)$ of the two approach tangents of the 20 curves. The table also shows the mean and standard deviation of speeds on each tangent being categorized into two types; independent and nonindependent tangents. 
Table 6-5: Speed on Approach Tangents of the 20 Curve.

\begin{tabular}{|c|c|c|c|c|c|c|c|}
\hline \multirow[b]{2}{*}{ Curve } & \multirow[b]{2}{*}{$R(\mathrm{~m})$} & \multirow[b]{2}{*}{ Direction } & \multirow[b]{2}{*}{$L_{T}(\mathrm{~m})$} & \multicolumn{2}{|c|}{ Non-independent Tangents } & \multicolumn{2}{|c|}{ Independent Tangents } \\
\hline & & & & $\mathrm{V} 50(\mathrm{~km} / \mathrm{h})$ & $\sigma(\mathrm{km} / \mathrm{h})$ & $\mathrm{V} 50(\mathrm{~km} / \mathrm{h})$ & $\sigma(\mathrm{km} / \mathrm{h})$ \\
\hline \multirow{2}{*}{1} & \multirow{2}{*}{388.00} & 1 & 809.27 & & & 92.00 & 9.21 \\
\hline & & 2 & 482.57 & & & 87.63 & 7.71 \\
\hline \multirow{2}{*}{2} & \multirow{2}{*}{436.59} & 1 & 5509.00 & & & 103.93 & 10.79 \\
\hline & & 2 & 1415.25 & & & 82.80 & 6.98 \\
\hline \multirow{2}{*}{3} & \multirow{2}{*}{873.19} & 1 & 1415.25 & & & 87.69 & 7.33 \\
\hline & & 2 & 555.17 & & & 91.91 & 9.91 \\
\hline \multirow{2}{*}{4} & \multirow{2}{*}{873.19} & 1 & 555.17 & & & 95.28 & 9.99 \\
\hline & & 2 & 1014.78 & & & 89.75 & 8.29 \\
\hline \multirow{2}{*}{5} & \multirow{2}{*}{873.19} & 1 & 101.37 & 79.58 & 12.27 & & \\
\hline & & 2 & 2330.35 & & & 90.80 & 10.98 \\
\hline \multirow{2}{*}{6} & \multirow{2}{*}{388.00} & 1 & 294.78 & & & 92.56 & 9.34 \\
\hline & & 2 & 170.85 & 88.32 & 8.34 & & \\
\hline \multirow{2}{*}{7} & \multirow{2}{*}{582.12} & 1 & 233.94 & & & 91.45 & 10.80 \\
\hline & & 2 & 16.27 & 88.74 & 9.42 & & \\
\hline \multirow{2}{*}{8} & \multirow{2}{*}{582.12} & 1 & 16.27 & 88.51 & 10.24 & & \\
\hline & & 2 & 751.41 & & & 90.18 & 9.16 \\
\hline \multirow{2}{*}{9} & \multirow{2}{*}{698.55} & 1 & 388.74 & & & 91.68 & 10.21 \\
\hline & & 2 & 2000.00 & & & 91.37 & 10.65 \\
\hline & & 1 & 1149.59 & & & 91.00 & 10.53 \\
\hline 10 & 436.59 & 2 & 409.89 & & & 88.84 & 10.52 \\
\hline & & 1 & 124.23 & 78.94 & 8.48 & & \\
\hline 11 & 317.52 & 2 & 912.30 & & & 91.72 & 10.92 \\
\hline & & 1 & 663.33 & & & 91.44 & 11.95 \\
\hline 12 & 317.52 & 2 & 124.23 & 90.97 & 12.05 & & \\
\hline & & 1 & 340.51 & & & 84.98 & 12.85 \\
\hline 13 & 582.13 & 2 & 49.04 & 93.16 & 12.92 & & \\
\hline & & 1 & 49.04 & 83.28 & 11.54 & & \\
\hline 14 & 388.08 & 2 & 239.01 & & & 88.16 & 8.36 \\
\hline & & 1 & 214.28 & & & 92.35 & 8.06 \\
\hline 15 & 436.59 & 2 & 588.53 & & & 101.09 & 18.48 \\
\hline & & 1 & 2000.00 & & & 99.77 & 10.14 \\
\hline 16 & 436.59 & 2 & 212.48 & & & 88.99 & 9.80 \\
\hline 17 & 34000 & 1 & 483.00 & & & 95.13 & 10.08 \\
\hline 17 & 349.00 & 2 & 7918.00 & & & 90.00 & 9.46 \\
\hline & & 1 & 1505.00 & & & 85.77 & 9.42 \\
\hline 18 & 367.66 & 2 & 118.00 & 92.92 & 10.43 & & \\
\hline 19 & 537.35 & 1 & 510.00 & & & 99.62 & 7.89 \\
\hline & & 1 & 2000.00 & & & 91.44 & 8.40 \\
\hline 20 & 635.05 & 2 & 510.00 & & & 90.72 & 7.88 \\
\hline & & & Average & 87.16 & 10.63 & 91.67 & 9.87 \\
\hline
\end{tabular}


An independent tangent is one that is long enough to allow drivers to reach a freeflow speed while a non-independent tangent is short enough $\left(L_{\mathrm{T}}<200 \mathrm{~m}\right)$ that drivers do not reach their desired speed before entering the following curve. The limiting value commonly used in the literature to differentiate between independent and nonindependent tangents is the length of $200 \mathrm{~m}$ (Lamm et al. 1999). Thus, a tangent less than $200 \mathrm{~m}$ long is considered non-independent, while tangents with length of $200 \mathrm{~m}$ or more are considered independent. Out of 39 tangents, a total of 30 were classified as independent while 9 are non-independent. Using SPSS regression option and utilizing only the non-independent tangents, neither V50 nor $\sigma$ had a significant relationship with $R$ and/or $L_{\mathrm{T}}$. Therefore, the average values of 87.16 and $10.63 \mathrm{~km} / \mathrm{h}$ were assumed as best estimates for V50 and $\sigma$, respectively. Similarly, mean values of 91.67 and $9.87 \mathrm{~km} / \mathrm{h}$ were recommended for independent tangents.

\subsection{2.c Speed estimation in SDES}

Using the abovementioned findings, SDES was coded to calculate the speed distribution at a certain road section by first detecting the location of the section and the type of the road stretch at this section. If the section is located on a tangent shorter than $200 \mathrm{~m}$, a mean speed and standard deviation of 87.16 and $10.63 \mathrm{~km} / \mathrm{h}$, respectively, are used. In addition, values of 91.67 and $9.87 \mathrm{~km} / \mathrm{h}$ are used for the mean speed and standard deviation on tangents longer than $200 \mathrm{~m}$. If a section is located on a circular or transition curve, the radius is used to predict the mean speed using the model presented in Table 6-4 while the standard deviation is taken as $10.85 \mathrm{~km} / \mathrm{h}$. 
As mentioned before, SDES can read pre-regenerated distributions that are prepared using other tools such as Matlab. For the purpose of the case study in hand, only one speed distribution, with a mean of $100 \mathrm{~km} / \mathrm{h}$ and standard deviation of $10 \mathrm{~km} / \mathrm{h}$, was first generated. This distribution is then adjusted at every road section to produce another distribution that matches a certain mean and standard deviation; based on the section location. The process is performed using Equation 6-1 that adjusts every single value $\left(v_{\mathrm{i}}\right)$ in the original distribution (V50 $=100$ and $\sigma=10 \mathrm{~km} / \mathrm{h})$ and calculates a new value $\left(v_{\mathrm{j}}\right)$ using a mean, V50, and standard deviation, $\sigma$.

$$
V_{j}=V(50)+\left(V_{i}-100\right) * \frac{\sigma}{10}
$$

Equation 6-1

where; $V_{\mathrm{j}}=$ adjusted speed value $(\mathrm{km} / \mathrm{h}) ; V_{\mathrm{i}}=$ original speed value $(\mathrm{km} / \mathrm{h}) ; \mathrm{V}(50)=$ targeted mean speed $(\mathrm{km} / \mathrm{h})$; and $\sigma=\operatorname{targeted~standard~deviation~}(\mathrm{km} / \mathrm{h})$.

\subsubsection{Road Surface Condition on Highway 61}

For RqSD calculations, the weather condition in the vicinity of Highway 61 was required to predict a realistic friction distribution. Relevant information was available on the Ontario weather page (2008) which hosts monthly weather conditions that were collected continuously at fixed points covering the Province of Ontario. Due to capacity limitations of the web site, data for only the year 2002 were available. Thunder Bay airport was selected as the closest station to the road segment under study. A total of 12 files were imported to Excel for the 12 months of 2002. Each file contained detailed records of the weather conditions on each day of the year. Table 6-6 shows a sample of the weather condition in May 2002 where the amount of precipitation and brief description were provided on each day. 
Although, the data covered every day of the year, the time duration (e.g., number of hours) that the precipitation had taken on each day was not reported. This piece of information was essential to calculate the time percentage of the year where the road surface was dry, wet, or covered with snow. Therefore, an assumption was made that the precipitation rate is constant and, given the amount of precipitation, the time can be estimated. For more clarification, the maximum amount of $35 \mathrm{~mm}$ found on October $4^{\text {th }}$ 2002 was assumed falling over the whole day ( 24 hours). Accordingly, the numbers of hours on other days have been calculated proportionally. Table 6-7 shows the summation of the number of hours that was calculated for each weather condition.

Table 6-6: Sample of the Weather Condition on Highway 61 for the Month of May.

\begin{tabular}{cccl}
\hline Month & Day & $\begin{array}{c}\text { Precipitation } \\
(\mathrm{mm})\end{array}$ & \multicolumn{1}{c}{ Weather } \\
\hline May & 1 & 0.5 & NONE/NA \\
& 2 & 0 & SNOW OR ICE PELLETS \\
& 3 & 0 & SNOW OR ICE PELLETS \\
& 4 & 0 & RAIN OR DRIZZLE \\
& 5 & 6.1 & RAIN OR DRIZZLE \\
6 & 0 & RAIN OR DRIZZLE/SNOW OR ICE PELLETS \\
7 & 0 & RAIN OR DRIZZLE \\
8 & 0.5 & RAIN OR DRIZZLE/SNOW OR ICE PELLETS \\
9 & 9.9 & FOG/RAIN OR DRIZZLE/THUNDER \\
10 & 0.5 & RAIN OR DRIZZLE/SNOW OR ICE PELLETS \\
11 & 0 & NONE/NA \\
12 & 0.5 & RAIN OR DRIZZLE \\
13 & 0 & RAIN OR DRIZZLE \\
14 & 0 & RAIN OR DRIZZLE \\
15 & 0 & RAIN OR DRIZZLE \\
16 & 0.5 & RAIN OR DRIZZLE/SNOW OR ICE PELLETS \\
17 & 0 & NONE/NA \\
18 & 0 & NONE/NA \\
19 & 0.5 & NONE/NA \\
20 & 0 & NONE/NA \\
21 & 0 & NONE/NA \\
22 & 0 & NONE/NA \\
23 & 0 & RAIN OR DRIZZLE/THUNDER \\
\hline
\end{tabular}


Table 6-7: Summary of Different Weather Conditions during the Year 2002.

\begin{tabular}{|c|c|c|c|c|}
\hline Condition & Hours & $\%$ & Surface Condition & $\%$ \\
\hline FOG & 1.33 & & & \\
\hline NONE/NA & 17.73 & 8.48 & Dry & 8.48 \\
\hline FOG/RAIN OR DRIZZLE & 24.53 & 11.73 & \multirow{5}{*}{ Wet Summer } & \multirow{5}{*}{68.21} \\
\hline FOG/RAIN OR DRIZZLE/THUNDER & 11.33 & 5.42 & & \\
\hline RAIN OR DRIZZLE & 65.40 & 31.28 & & \\
\hline RAIN OR DRIZZLE/HAIL/THUNDER & 0.33 & 0.16 & & \\
\hline RAIN OR DRIZZLE/THUNDER & 41.00 & 19.61 & & \\
\hline $\begin{array}{l}\text { FOG/RAIN OR DRIZZLE/SNOW OR ICE } \\
\text { PELLETS }\end{array}$ & 0.33 & 0.16 & \multirow{4}{*}{ Snow Winter } & \multirow{4}{*}{23.31} \\
\hline FOG/SNOW OR ICE PELLETS & 0.67 & 0.32 & & \\
\hline $\begin{array}{l}\text { RAIN OR DRIZZLE/SNOW OR ICE } \\
\text { PELLETS }\end{array}$ & 20.00 & 9.57 & & \\
\hline SNOW OR ICE PELLETS & 27.73 & 13.27 & & \\
\hline
\end{tabular}

The first row in this table, which represents the fog conditions, was first excluded since driving at such time could force drivers to operate in a different manner that is out of the scope of this research. In addition, the description none/NA was assumed to describe dry surfaces. Moreover, a description containing the word snow or ice was assumed to describe a snow condition while others could be more probably describing wet surfaces. Using these assumptions, the summation of the percentages for dry, wet, and snow conditions were calculated as $8.48,68.21$, and $23.31 \%$, respectively. However, these percentages were considered unrealistic, especially with the notable low percentage of the dry condition $(8.48 \%)$.

Therefore, another approach to obtain the weather condition was tried considering the data posted on the weather network web site. As shown in Table 6-8, these data included the mean values of several meteorological parameters such as rain and snow. The data cover a 30-year time period from 1961 to 1990 . Although the data should be reliable with the long time period the data are covering, the conversion of such data to 
percentages of different road conditions was not possible. Attempts to obtain more details using contact information available for public have also failed. With the difficulty to obtain further information, the existing information was not appropriate for the purpose of the analysis to be carried out in this thesis. Therefore, three percentages were assumed to be 60,25 , and $15 \%$ for the dry, wet, and snow conditions, respectively. However, accurate information is needed for precise results and reliable hazard assessments in the future.

Table 6-8: Mean Values of Several Meteorological Parameters as Posted in The Weather Network Web Site.

\begin{tabular}{|c|c|c|c|c|c|c|c|c|c|c|c|c|}
\hline $\begin{array}{c}\text { Number of days } \\
\text { where }\end{array}$ & 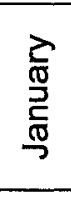 & 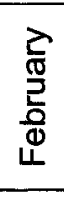 & $\frac{\frac{c}{0}}{\frac{0}{0}}$ & $\overline{\bar{\alpha}}$ & 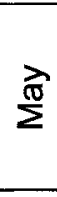 & 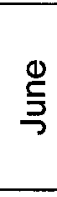 & $\grave{3}$ & $\begin{array}{l}\overline{0} \\
\stackrel{5}{8} \\
\frac{9}{2}\end{array}$ & 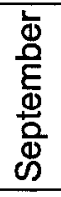 & $\begin{array}{l}\text { D } \\
\stackrel{0}{0} \\
\stackrel{0}{0} \\
0\end{array}$ & 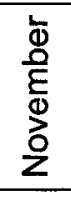 & $\begin{array}{c}\xi \\
\stackrel{亏}{\circ}\end{array}$ \\
\hline Temp. $<=0^{\circ} \mathrm{C}$ & 31 & 28 & 30 & 24 & 11 & 0 & 0 & 0 & 5 & 17 & 27 & 173 \\
\hline Rain $>=0.2 \mathrm{~mm}$ & 0 & 0 & 3 & 7 & 11 & 13 & 12 & 13 & 13 & 10 & 5 & 87 \\
\hline Rain >=5 mm & 0 & 0 & 1 & 2 & 4 & 5 & 5 & 5 & 5 & 4 & 2 & 33 \\
\hline Rain $>=10 \mathrm{~mm}$ & 0 & 0 & 0 & 0 & 2 & 3 & 3 & 3 & 2 & 2 & 0 & 15 \\
\hline Rain >=25 mm & 0 & 0 & 0 & 0 & 0 & 0 & 0 & 0 & 0 & 0 & 0 & 0 \\
\hline Snow $>=0.2 \mathrm{~cm}$ & 13 & 10 & 9 & 4 & 0 & 0 & 0 & 0 & 0 & 2 & 9 & 47 \\
\hline Snow $>=5 \mathrm{~cm}$ & 3 & 2 & 2 & 0 & 0 & 0 & 0 & 0 & 0 & 0 & 2 & 9 \\
\hline Snow $>=10 \mathrm{~cm}$ & 0 & 0 & 0 & 0 & 0 & 0 & 0 & 0 & 0 & 0 & 0 & 0 \\
\hline Snow $>=25 \mathrm{~cm}$ & 0 & 0 & 0 & 0 & 0 & 0 & 0 & 0 & 0 & 0 & 0 & 0 \\
\hline Precip. $>=0.2 \mathrm{~mm}$ & 12 & 10 & 11 & 10 & 11 & 13 & 12 & 13 & 13 & 11 & 11 & 127 \\
\hline Precip.>=5 mm & 2 & 2 & 3 & 3 & 5 & 5 & 5 & 5 & 5 & 4 & 3 & 42 \\
\hline Precip. $>=10 \mathrm{~mm}$ & 0 & 0 & 1 & 2 & 2 & 3 & 3 & 3 & 2 & 2 & 1 & 19 \\
\hline Precip. $>=25$ mm & 0 & 0 & 0 & 0 & 0 & 0 & 0 & 0 & 0 & 0 & 0 & 0 \\
\hline Snow Cover $>=1 \mathrm{~cm}$ & 31 & 28 & 27 & 8 & 0 & 0 & 0 & 0 & 0 & 0 & 11 & 105 \\
\hline Snow Cover $>=5 \mathrm{~cm}$ & 30 & 28 & 24 & 6 & 0 & 0 & 0 & 0 & 0 & 0 & 6 & 94 \\
\hline Snow Cover $>=10 \mathrm{~cm}$ & 28 & 27 & 20 & 4 & 0 & 0 & 0 & 0 & 0 & 0 & 3 & 82 \\
\hline Snow Cover $>=20 \mathrm{~cm}$ & 23 & 22 & 16 & 3 & 0 & 0 & 0 & 0 & 0 & 0 & 1 & 65 \\
\hline Snow Cover $>=50 \mathrm{~cm}$ & 6 & 5 & 4 & 0 & 0 & 0 & 0 & 0 & 0 & 0 & 0 & 15 \\
\hline
\end{tabular}

The weather statistics displayed here represent the mean value of each meteorological parameter for each month of the year. The sampling period for this data covers 30 years from 1961 to 1990. 


\subsubsection{Sight Distance and POH Profiles}

In this section, the developed speed model along with the recommendations of the speed distributions on tangents and curves will be used to assess POH. First, the selection of a proper number of simulations is discussed in Section 6.2.4.a. Then, Section 6.2.4.b will present the RqSD, AvSD, and $\mathrm{POH}$ profiles and main findings will be highlighted.

\subsection{4.a Number of simulations}

As the precision of the $\mathrm{POH}$ profile would depend on the number of Monte Carlo simulations, it was important to calculate an appropriate number, $N$, required for the hazard assessment of the case study in this chapter. It is worthy to mention that the 100000 iterations used before in CHAPTER 4 and CHAPTER 5 showed consistent results with the analytical reliability technique. However, the use of the same number in this section will be difficult and a simulation could require considerably long time to be run on the $8-\mathrm{km}$ road segment. This time duration was estimated by running samples with lower values of $N$ on only $1 \mathrm{~km}$ of the road.

In general, several methods were reported in relevant textbooks to calculate such number. For example, it was mentioned in Harr (1925) that each trial of the simulation is an experiment with a certain probability of success; with all trials being independent and forming a binomial distribution. When the number of trials is large, normal approximation of the binomial distribution can be used. After some algebra, a formula was presented as follows: 


$$
N=\left(\frac{Z_{\alpha / 2}^{2}}{4 \alpha^{2}}\right)^{m}
$$

Equation 6-2

where; $N=$ number of Monte Carlo simulations; $Z=$ standard deviation corresponds to $\alpha / 2 ; \alpha=$ maximum allowable error; and $m=$ number of variables.

In other words, if $99 \%$ confidence is selected $\left(1-\alpha=0.99 ; Z_{\alpha / 2}=2.58\right)$, the required number of iteration using one variable would be:

$$
N=\frac{2.58^{2}}{4 * 0.01^{2}}=16,641
$$

This number, however, will increase dramatically using few variable in the simulation like the case of simulating the RqSD with three variables (e.g., $N=(16,641)^{3}$ $=4608.27 * 10^{9}$ ). For the purpose of this research, however, another approach was followed to identify an appropriate and more practical estimate of $N$. First, the profile of POH was generated using only 1000 iterations. Then, the road section $(14+100)$ that had the highest $\mathrm{POH}$ of 0.019 was selected for further evaluation using different higher values of $N$; including $5000,10000,25000,50000$. It should be mentioned that, for simplicity, only acceleration rate was used to calculate RqSD in this preliminary step assuming high friction coefficients to accommodate such rates. As shown in Table 6-9, $\mathrm{POH}$ at station $14+100$ was found to have the same value after 10000 iterations and hence this number was selected for final application. Nevertheless, increasing this number may enhance the precision and should be considered if possible. 
Table 6-9: POH using Different Numbers of Iterations.

\begin{tabular}{cc}
\hline Number of iterations & POH @ Station $14+100$ \\
\hline 1,000 & 0.019 \\
5,000 & 0.014 \\
10,000 & 0.015 \\
25,000 & 0.015 \\
50,000 & 0.015 \\
\hline
\end{tabular}

\subsection{4.b Hazard assessment}

Before running SDES, the friction distribution was first created using the three percentages $(60,25,15 \%$ for dry, wet, and snow, respectively) assumed in Section 6.2.3. Considering 10000 total number of iterations, the friction distribution was formed of three smaller ones in terms of their dimensions. The characteristics of these three distributions are presented in Table 6-10. The table shows that a 6000 -value distribution was generated by Matlab for the dry condition with a mean of 0.907 and standard deviation of 0.045 . Similarly, two other distributions were created for the wet and snow conditions using the dimension, mean friction, and standard deviation listed in this table. These three distributions were combined and the resulting one was considered for RqSD calculations as described back in Section 4.4. Other distributions for the rest of parameters were also used as recommended before in this research (refer back to Table 4-2 and Table 5-1); except for the speed distribution that was generated using a mean and standard deviation of 100 and $10 \mathrm{~km} / \mathrm{h}$, respectively. As mentioned earlier, the speed distribution at each point was then adjusted based on whether the point was on a curve, independent tangent, or non-independent tangent. 
Table 6-10: Characteristics of the Friction Distribution.

\begin{tabular}{cccc}
\hline \multirow{2}{*}{ Condition } & \multirow{2}{*}{ Dimension } & \multicolumn{2}{c}{ Friction Coefficient } \\
\cline { 3 - 4 } & & mean & $\sigma$ \\
\hline Dry & 6000 & 0.907 & 0.045 \\
Wet & 2500 & 0.730 & 0.100 \\
Snow & 1500 & 0.260 & 0.050 \\
\hline
\end{tabular}

After generating and importing the parameters' distributions to SDES, the upper limit of the sight distance was set to be $500 \mathrm{~m}$. An AvSD more than this value was considered too high to induce hazard due to insufficient sight distance. In addition, although the $8-\mathrm{km}$ road segment was entered to SDES, only $6 \mathrm{~km}$ were analyzed excluding the first and last $1 \mathrm{~km}$. This is because, near both ends of the $8-\mathrm{km}$ segment, AvSD and RqSD were found exceeding the limits of the segment in some cases. Subsequently, SDES displayed error messages. Therefore, only the road segment from Station $11+000$ to $17+000$ was considered. The simulation run of this segment took almost two weeks using a computer with a 2.4-GHz Opteron Processor and 3-GB Ram. The results were finally presented in Figure 6-5, where six separate graphs were provided for individual 1-km segments. The top two rectangles of each graph contain the RqSD, AvSD, and POH profiles on the left and right lanes while the vertical and horizontal alignments are displayed in the third and forth rectangles, respectively.

The results indicated that the lowest mean AvSD value of $179.25 \mathrm{~m}$ took place on the right lane at Station $13+900$ while the lowest value on the left lane was $181.51 \mathrm{~m}$ at Station $13+200$. In addition, a minimum mean RqSD of $114.592 \mathrm{~m}$ was observed at Station $13+400$ on the left lane whereas $116.432 \mathrm{~m}$ was found on the right lane at Station $16+600$. 

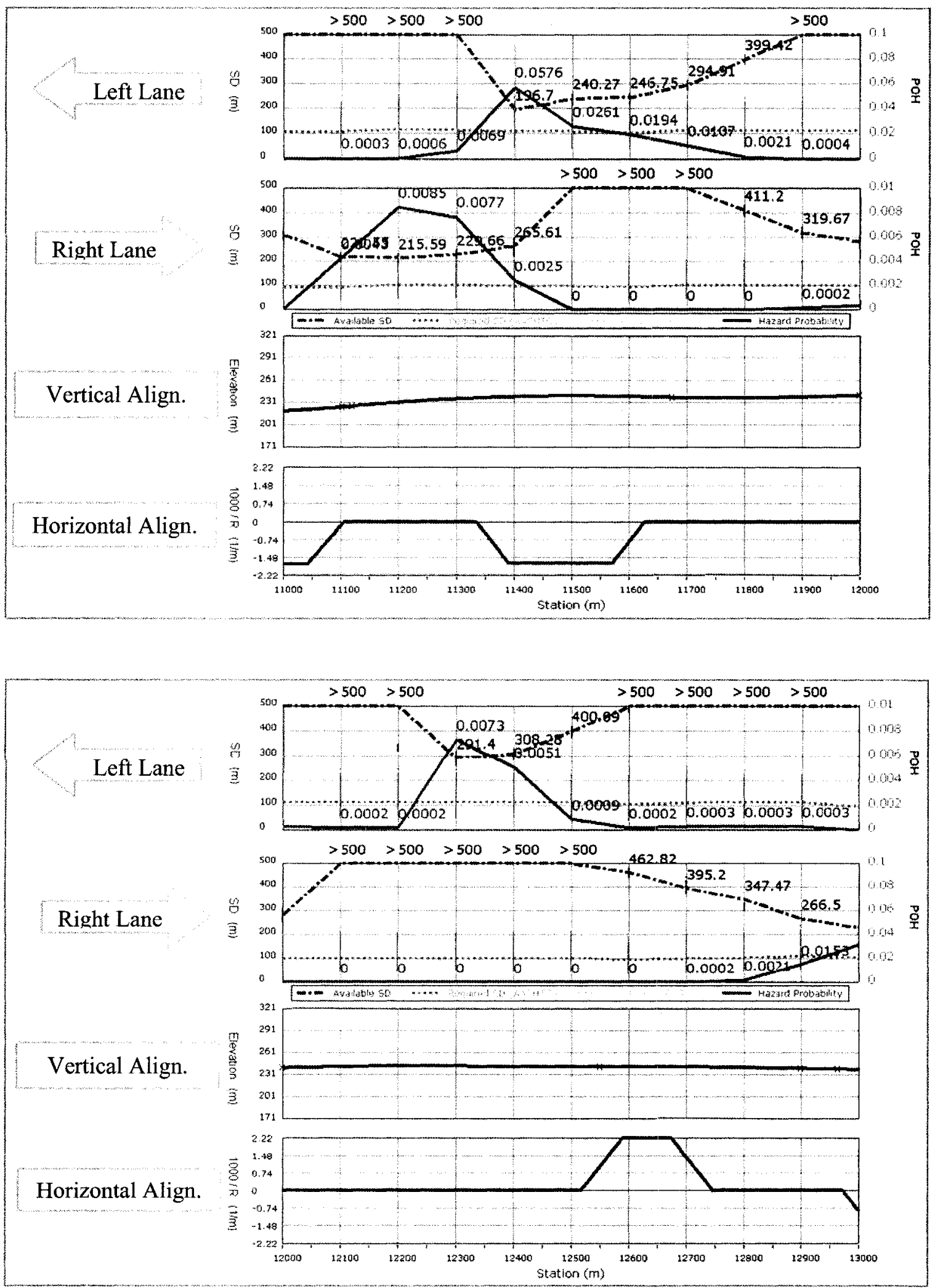

Figure 6-5: POH Profiles [Station $11+000$ to Station 13+000]. 

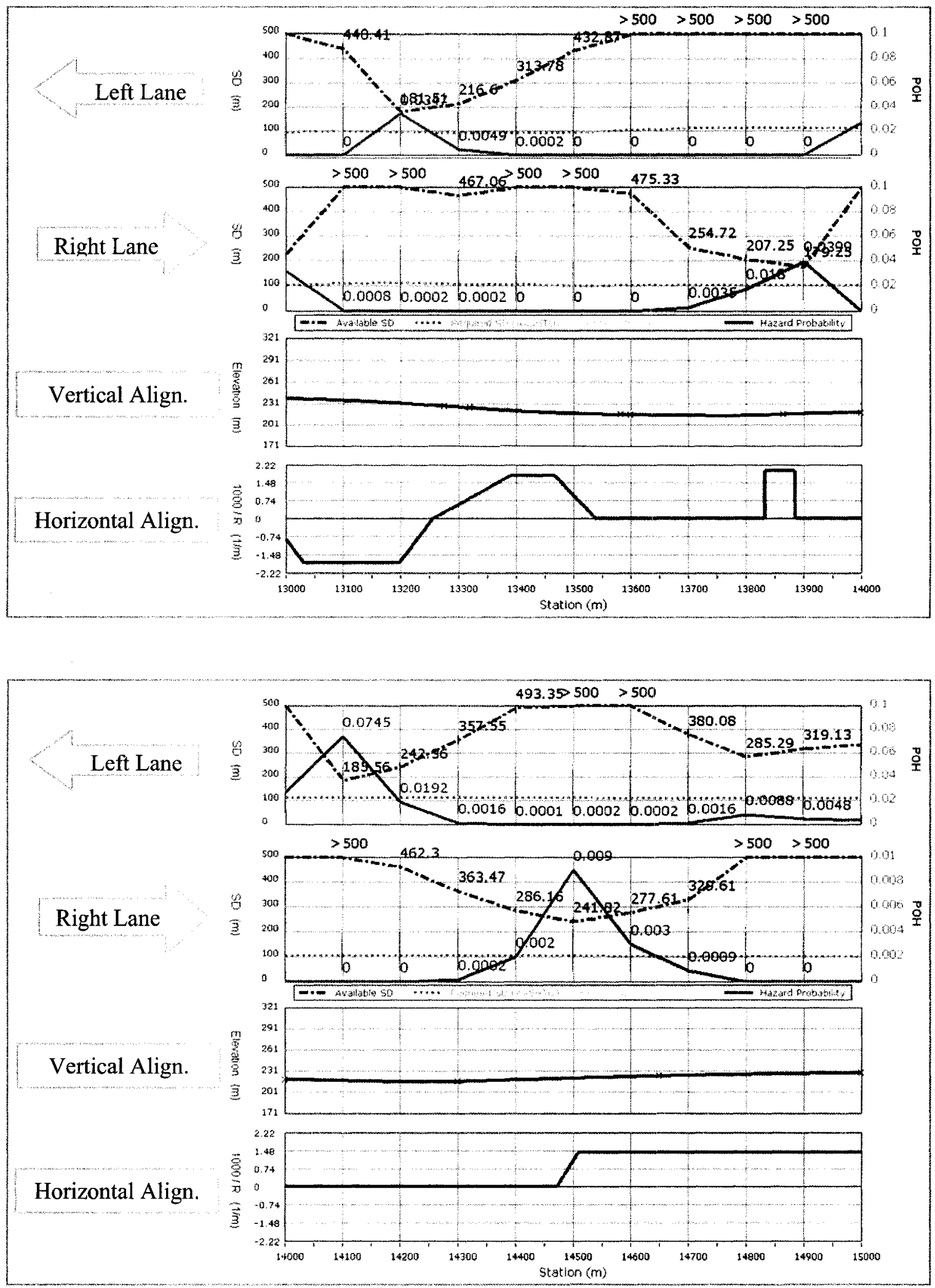

Figure 6-5: POH Profiles (continued) [from Station 13+000 to Station 15+000]. 

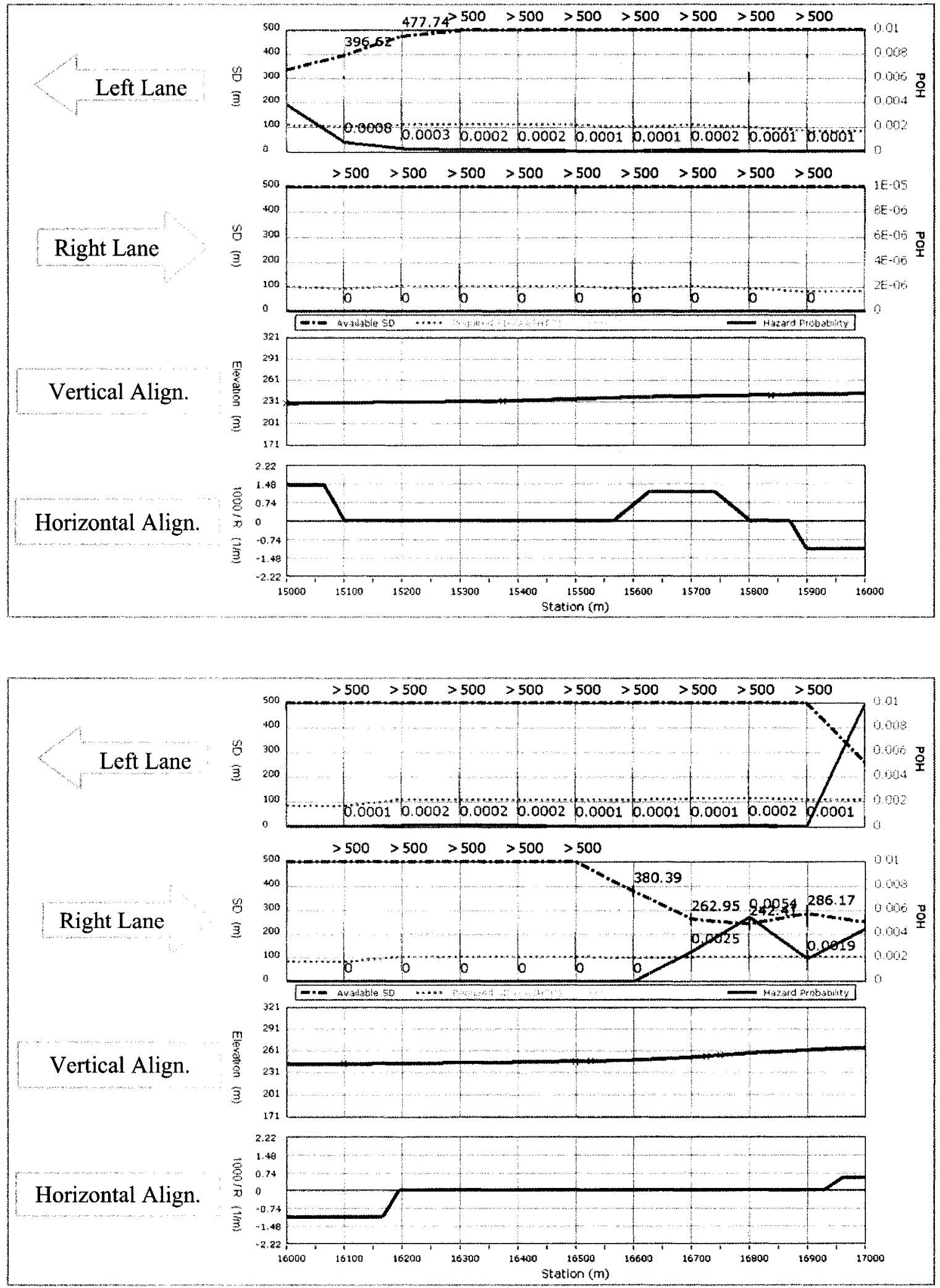

Figure 6-5: POH Profiles (continued) [Station $15+000$ to Station 17+000]. 
Moreover, the maximum mean RqSD is $139.792 \mathrm{~m}$ on the right lane at Station $13+200$ and $137.762 \mathrm{~m}$ on the left lane at Station $11+200$. For the hazard profile, a POH lower than $0.0005(0.05 \%)$ was obtained along most of the road segments on both directions. In addition, only four sections indicated hazard levels higher than $0.01(1 \%)$. As shown in Table 6-11, the highest value of $0.0745(7.45 \%)$ on the left lane at Station 14+100 may indicate that $7.45 \%$ of drivers will suffer insufficient sight distance, if the need arises to perform an emergency stopping manoeuvre. This may require either increasing the AvSD at that section or avoiding any surprises to drivers shortly after.

Table 6-11: Road Sections with POH Greater Than $1 \%$.

\begin{tabular}{ccc}
\hline Direction & Station & POH \\
\hline Left & $14+100$ & 0.0745 \\
Left & $11+400$ & 0.0576 \\
Right & $13+900$ & 0.0399 \\
Left & $13+100$ & 0.0347 \\
\hline
\end{tabular}

\subsection{Probability of Collision}

It is important to shed light on the difference between $\mathrm{POH}$ and probability of collision (POC). As mentioned before, $\mathrm{POH}$ indicates a percentage of road users whose AvSD is less than RqSD. Those drivers would be exposed to a higher chance of collisions than normal. However, the occurrence of a collision is always contingent on an instance. Indeed, a section that limits the sight distance may practically experience no collisions if the driver did not have to stop to avoid an imminent collision. As an example, and in case AvSD is less than RqSD for a speedy driver, nothing would happen until the driver is required to stop to avoid hitting an object along the vehicle's path. Since the probable existence of an instance would increase the chance of a collision, POC can hence be 
expressed as shown in Equation 6-4. The equation indicates that an instance such as a fallen tree, an existing rock, or more frequently an extended traffic queue could contribute to increase POC.

$$
P O C=P O H * P O I
$$

Equation 6-4

where; $P O C=$ probability of collision; $P O H=$ probability of hazard; $P O I=$ probability of instance.

\subsection{Summary}

In this chapter, a real case study was used to show the application of the reliability analysis to evaluate the hazard due to insufficient sight distance. The analysis was performed on an $8-\mathrm{km}$ road segment along Highway 61 located in Ontario. Speed data were also used to model the speed distribution along horizontal curves and tangents of two-lane roadways. In addition, attempts were considered to obtain real data that would describe the weather conditions near Highway 61. Findings were used to calculate a more realistic RqSD profile that also considered the change in the longitudinal grade. Using 10000 iterations, SDES was run and three profiles were finally generated for RqSD, AvSD, and $\mathrm{POH}$. The $\mathrm{POH}$ profile indicated that only four sections had relatively high hazard levels between $3.4 \%$ and $7.5 \%$. However, it was stressed that the probability of collision could still be low if drivers are not required to urgently stop at those sections. 


\section{CHAPTER 7}

\section{Contribution to Design Practice}

In this chapter, a reliability-based approach was developed to locate lateral obstructions along horizontal curves considering the 3D alignment. The approach uses the probability of hazard as a main criterion in the design process. The methodology is also beneficial for a hazard evaluation of an existing design where a lateral obstacle restricts AvSD on a horizontal curve. In Section 7.1, the design methodology in current design guides is presented while the weaknesses of that methodology are highlighted in Section 7.2. In Section 7.3 , a new reliability-based methodology was developed to calculate the lateral clearance along 3D alignments. In addition, a design aid was prepared in Section 7.4 using the new methodology to facilitate the design of lateral clearance on a curve with a 400- $\mathrm{m}$ radius as an example. Another design aid was developed in 7.5 for hazard evaluation of existing designs. Finally, the interrelation between speed and AvSD is discussed in Section 7.6.

\subsection{Current Design Methodology}

The lateral clearance along a horizontal curve is determined in the current design guides in terms of the design speed. The chart presented in Figure 7-1 shows a schematic diagram of the current design approach presented in both AASHTO (2004; Exhibit 5-53) and TAC (1999; Figure 2.1.2.11). 


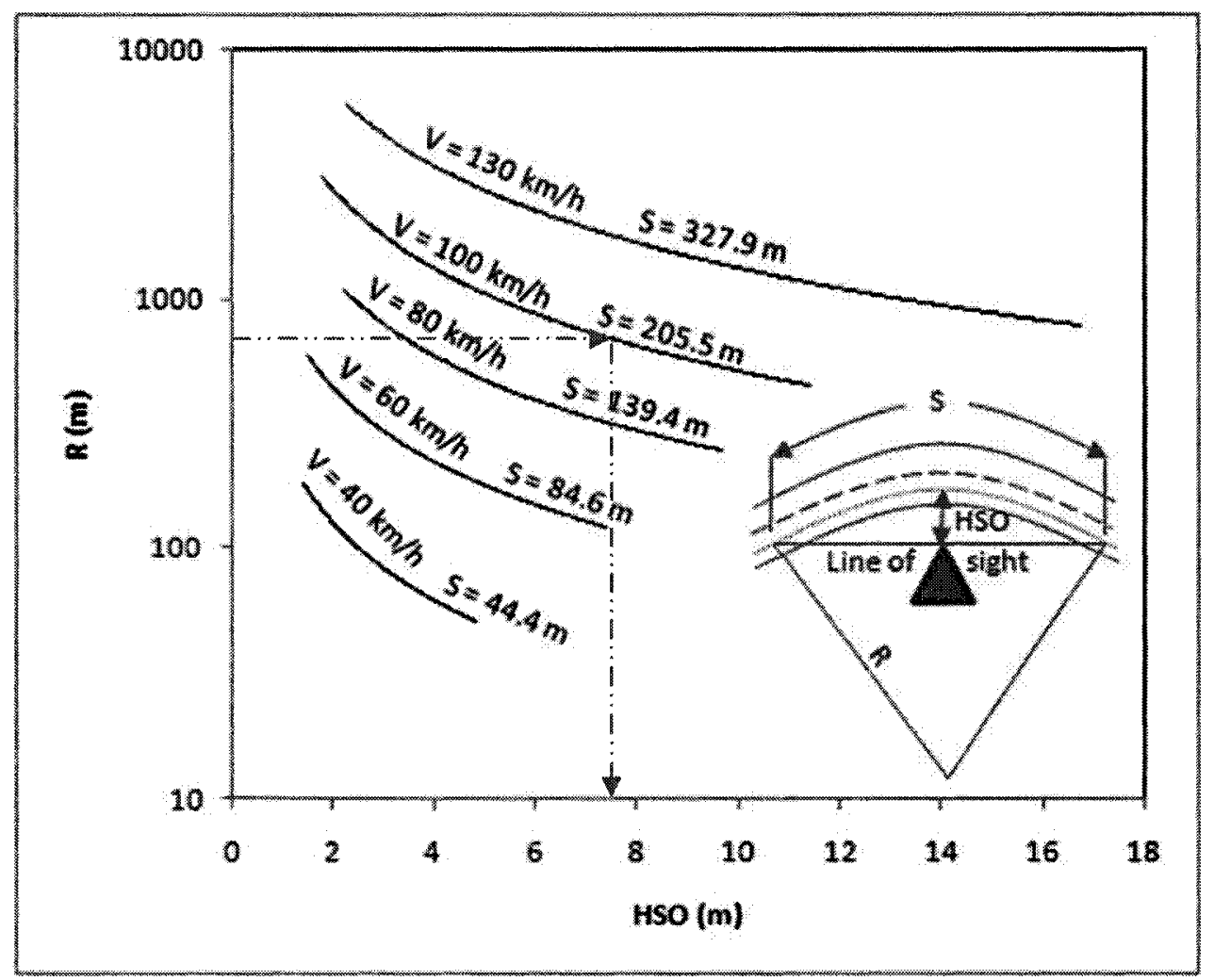

Figure 7-1: Determination of Lateral Clearance using Current Method (Adapted from TAC 1999).

The methodology assumes a case of a flat horizontal curve on which drivers have their line of sight restricted by an obstruction located at a distance HSO from the centerline of the inner lane. The figure uses the analytic geometry of the sector to relate the curve radius $(R)$ to the lateral clearance (HSO) given a specific arc length (AvSD, $S$ ). The traditional design steps start by calculating the curve radius using the mass-point equation presented earlier in Equation 4-3, which satisfies the vehicle stability. Then, either the chart or the equation used to prepare the chart (refer back to Equation 2-7) is used to determine $H S O$. Using TAC (1999) guidelines, the road cross-section is designed such that $H S O$ is provided at an average height of $0.72 \mathrm{~m}$ (average of 1.05 and $0.38 \mathrm{~m}$ for 
$h_{1}$ and $h_{2}$, respectively). In AASHTO (2004), a different height of $0.84 \mathrm{~m}$ is used as an average between 1.08 and $0.6 \mathrm{~m}$ for $h_{1}$ and $h_{2}$, respectively.

\subsection{Critique of Current Methodology}

One of the main drawbacks of the existing design approach is that it considers the horizontal alignment assuming a negligible effect of the vertical curvatures. Indeed, only road segments with lateral obstructions that have a vertical edge would make the approach applicable regardless of the configuration of the vertical alignment. However, any non-vertical side slope would limit the validity of the chart to only those curves with constant longitudinal grades. Therefore, the design of a scenario where a non-vertical side slope exists on a horizontal curve overlapping with a vertical curve could overestimate or underestimate the road supply and, subsequently, the design might become hazardous to road users or uneconomic.

For more explanation in this regard, one needs to more closely examine the point where the sight line is obstructed by a lateral obstruction. As shown in Figure 7-2, this point is located at a certain height $\left(h_{\mathrm{O}}\right)$ from the road surface at the mid-section of AvSD. In cases of constant longitudinal grades, this height is the average of both the driver eye and object heights; assuming a little effect of the change in the pavement cross slopes along AvSD. However, the value $h_{0}$ would intuitively decrease or increase with the implementation of either a crest or sag vertical curves, respectively. If an obstacle such as a building has a vertical edge, the change in $h_{\mathrm{O}}$ would have no effect on HSO. However, in case of a non-vertical side slope, the value of $H S O$ would change with the change of $h_{\mathrm{O}}$ while the point of tangency slides up and down on the slopped surface. 


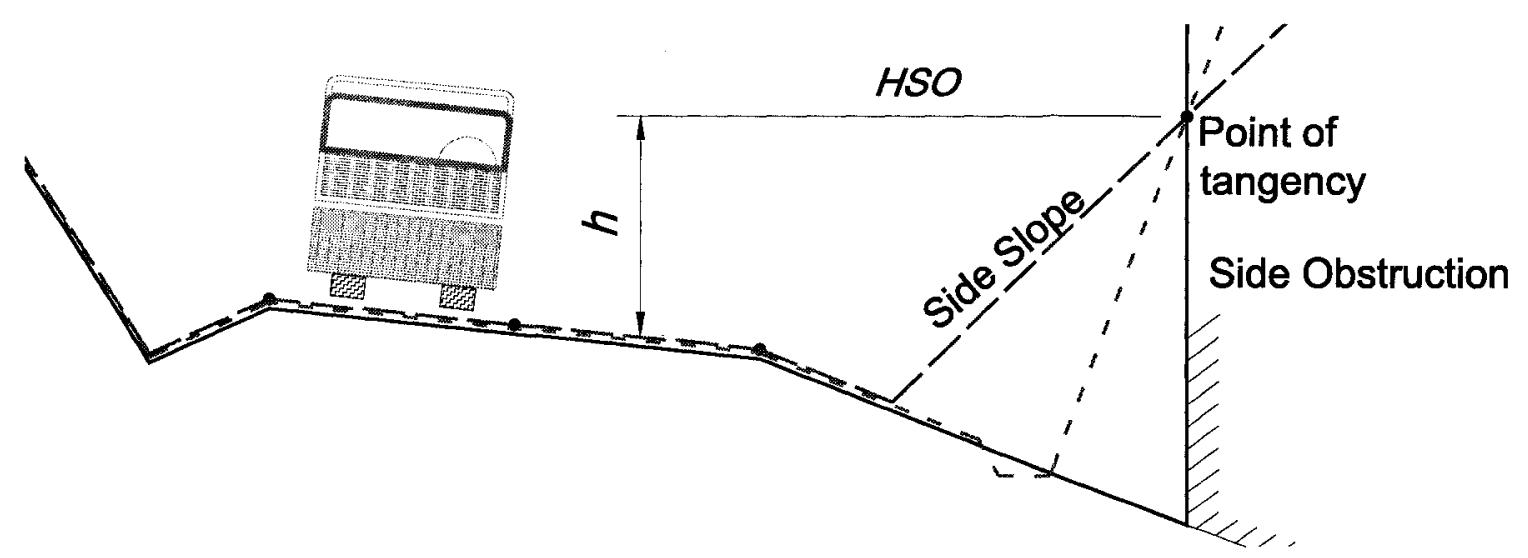

Figure 7-2: Different Cross-Sections at the Mid-Point of AvSD.

Two terms are hence suggested in this research to distinguish between the inaccurate $H S O$ obtained from the chart, which would be referred to as $2 \mathrm{D}-\mathrm{HSO}$, and the actual value, which would be referred to as 3D-HSO. The difference between 2D-HSO and 3D-HSO would depend on several factors including the type of the vertical curve (crest or sag), the rate of vertical curvature $(1 / K)$, the side slope, and other factors that define the value of 2D-HSO such as lane width, shoulder width, and lane and shoulder cross slopes. In addition, because the current chart was built using the deterministic concept, AvSD is calculated based solely on the geometry of the road alignment, and then the minimum design is achieved when AvSD is equal to RqSD. Due to the accumulated safety margins considered for all design parameters, 2D-HSO may be conservative and the use of a relatively lower $H S O$ value could still be acceptable with the recognition and quantification of the expected hazard. As an alternative, a risk-based design methodology should include the variation of both AvSD and RqSD parameters. This approach would be more realistic and would consider the driver characteristics in the design process. In addition, this would even give more opportunities for cost/benefit analysis. 
Furthermore, a note was placed in the chart presented in TAC (1999) informing that the chart does not include the correction for loss of friction on sharp curves. In this regard a separate table was provided to compensate for such reduction by increasing 2DHSO by more than $15 \%$ in some cases (see Table $7-1$ ). On one hand, this correction would make the design even more conservative. Furthermore, the design could become uneconomic if corrections were also made to consider the increase in SSD due to a longitudinal downgrade, which may have even more impact than the influence of horizontal curvature as was described before in Section 4.1.

Table 7-1: Sample Correction of Lateral Clearance on Sharp Curves (Adapted from TAC 1999).

\begin{tabular}{ccccc}
\hline $\begin{array}{c}\text { Design Speed } \\
(\mathrm{km} / \mathrm{h})\end{array}$ & $\mathrm{e}_{\max }$ & $\begin{array}{c}\text { HSO considering } \\
\text { loss of friction }\end{array}$ & $\begin{array}{c}\text { HSO neglecting } \\
\text { loss of friction }\end{array}$ & Difference (\%) \\
\hline 40 & & 4.41 & 4.06 & 7.9 \\
50 & 5.43 & 4.89 & 9.9 \\
60 & 6.67 & 5.93 & 11.1 \\
70 & 0.04 & 8.95 & 7.63 & 14.7 \\
80 & & 10.19 & 8.63 & 15.3 \\
90 & & 10.61 & 9.32 & 12.2 \\
100 & 12.16 & 10.68 & 12.2 \\
\hline 40 & 4.80 & 4.42 & 7.9 \\
50 & & 6.04 & 5.42 & 10.3 \\
60 & & 7.71 & 6.82 & 11.5 \\
70 & 9.43 & 8.02 & 14.9 \\
80 & & 11.44 & 9.65 & 15.6 \\
90 & 11.84 & 10.41 & 12.1 \\
100 & & 13.46 & 11.89 & 11.7 \\
110 & 0.06 & 13.88 & 12.62 & 9.1 \\
120 & & 15.10 & 13.56 & 10.2 \\
130 & & 15.02 & 14.11 & 6.0 \\
\hline
\end{tabular}




\subsection{New 3D Reliability-Based Design Methodology}

The proposed modification in this section adds a third dimension to the existing chart and allows for hazard assessment based on the reliability analysis. Using the new approach, $H S O$ is calculated using a pre-determined value of $\mathrm{POH}$. For more illustration, Figure 7-3 shows a framework that describes the reliability-based design methodology. The methodology takes into account the parameters influencing both AvSD and RqSD. For AvSD, two possibilities are considered for obstructions of the line of sight including (1) horizontal obstruction by a lateral obstacle such as a side slope at a horizontal curve located in a cut section and (2) vertical obstruction by a road surface due to the vertical curvature of a crest curve.

Therefore, a total of seven factors were displayed in the right side of this framework. Five parameters out of these seven are constants describing the road parameters including the lateral offset $(H S O)$, horizontal curve radius $(R)$, vertical curve length $(L)$, and longitudinal grades of vertical curve tangents $\left(g_{1}, g_{2}\right)$. In addition, two other factors may cause variation in the AvSD; namely the driver eye height $\left(h_{1}\right)$ and object height $\left(h_{2}\right)$. As for the RqSD, there are four parameters that can be expressed using distributions rather than constant values which are speed $(V)$, PR time $(t)$, friction coefficient $(f)$, and deceleration rate $(a)$. Using these factors, the proposed methodology to calculate the $3 \mathrm{D}$ reliability-based $H S O$ can be applied as follows: 


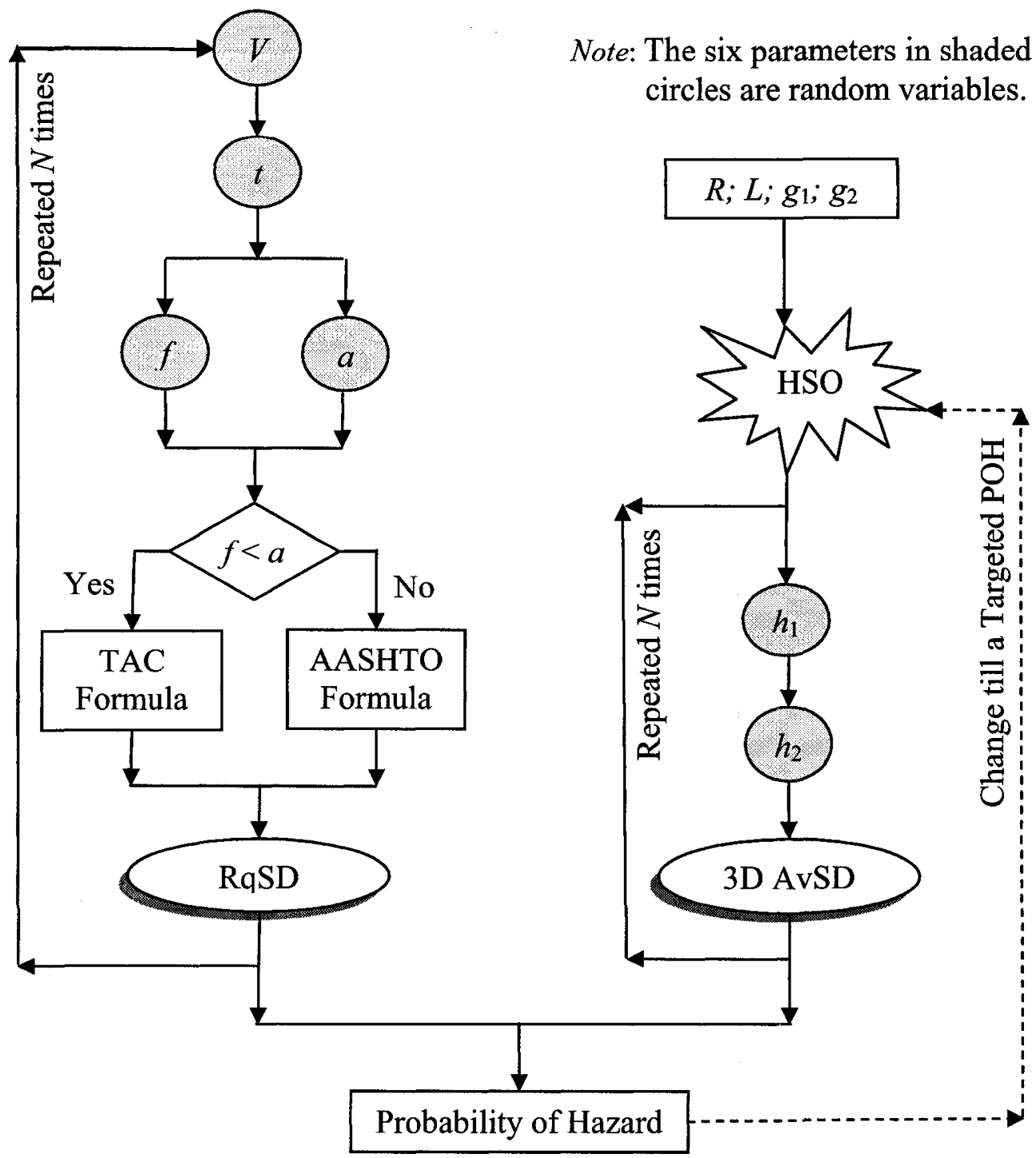

where; $V=$ operating speed; $t=$ perception and reaction time; $f=$ friction coefficient; $a=$ deceleration rate; $H S O=$ horizontal sight offset; $R=$ horizontal curve radius; $L=$ vertical curve length; $g_{1}$ and $g_{2}=$ longitudinal grade of vertical curve tangents; $h_{1}=$ driver eye height; and $h_{2}=$ object height.

Figure 7-3: A Layout of the Proposed Design Methodology.

1. Starting from the top right side of the suggested framework, the horizontal curve radius and vertical curve length are calculated using the traditional methods in current design guides. 
2. An initial value of $H S O$ can be assumed. Current design method could be taken as a guide in this regard.

3. Two distributions for $h_{1}$ and $h_{2}$ are generated with $N$ dimension.

4. Using a set of values for $h_{1}$ and $h_{2}$, the 3D AvSD is determined considering the combined horizontal and vertical road alignments. The process is repeated $N$ times forming AvSD distribution.

5. On the other side, a speed distribution is generated as a function of the curve radius using available models in the literature. The mean value of AvSD could also be considered in the speed estimation if information relating expected speed to AvSD is available.

6. Two other distributions are generated for $t$ and $a$.

7. Information about the weather condition during an average year is collected. A distribution of $f$ is generated accordingly to reflect the expected road surface condition.

8. Using $a$ and $f$ distributions, two values are picked at a time. If the selected $f$ is lower than the selected $a$, the TAC formula is used to calculate RqSD. Otherwise, the AASHTO formula is used. Using either formula, the simulated RqSD is calculated as described earlier in CHAPTER 4. Similar to the case of AvSD, the process is repeated for $N$ times forming RqSD distribution.

9. Individual values of AvSD and RqSD distributions are evaluated and cases where AvSD is less than RqSD are isolated. The percentage of those cases to the $N$ trials represents the $\mathrm{POH}$. 
10. The HSO is changed as necessary and steps 4 and 9 are repeated till a predetermined $\mathrm{POH}$ is reached.

The advantage of the new methodology is that it allows more recognition of the variation in the driver behaviour. This is achieved in the analysis by including distributions rather than single values for the six design parameters listed above. The methodology also considers the 3D alignment in calculating both AvSD and RqSD and a design should hence be more realistic compared to the current method. Furthermore, the methodology utilizes the $\mathrm{POH}$ that reflects the percentage of those drivers operating at risk. The use of this measure may help reduce the potential of collisions

\subsection{Establishment of a Hazard-Based Design Aid}

In the steps presented earlier, a user is required to calculate the 3D AvSD rather than following the current $2 \mathrm{D}$ approach. In addition, an accurate RqSD through simulating a vehicle attempting to stop is also recommended to account for changes in both longitudinal grade and friction along the braking distance. Given that no analytical methods are available to do these requirements and taking the advantage of SDES to perform them efficiently, a tool can be prepared for direct use. In this regard, a set of charts can be developed as design aids, where each chart is valid for a certain radius. The procedure that can be used to prepare the tool and the development of a design aid using such procedure are described in the following sub-sections. 


\subsubsection{Procedures and Assumptions}

In this section, steps are presented to develop a tool for the determination of $H S O$ using the new 3D reliability-based approach presented earlier in this chapter. The tool would include a set of charts where each chart is valid for a certain curve radius combined with a range of vertical curves. The procedure suggested to prepare one chart is described as follows:

1. Assume a $1-\mathrm{km}$ road segment that consists of a simple horizontal curve located at a cut section and has a certain radius $(R)$.

2. The road can be assumed to have a simple cross section with a $3.6-\mathrm{m}$ lane width, $8 \%$ superelevation, $8 \%$ shoulder slope, and $2 \mathrm{H}: 1 \mathrm{~V}$ upward side slope. Different values of shoulder width may be taken with a range from 0 to $20 \mathrm{~m}$ to yield different values of $H S O$. It should be noted that a configuration of the cross section has to be assumed to run SDES. However, in order to reduce the number of variables in the design chart, the chart will utilize the parameter $H S O$ such that the configuration and type of the cross section would not have a significant effect on the developed tool.

3. Referring back to Equation 5-3, the mean 2D-HSO can be calculated for each value of shoulder width using mean values of two $h_{1}$ and $h_{2}$ distributions. The most critical 2D-HSO can be obtained on the middle portion of the road segment where both the driver eye and object are located on the horizontal curve. 
4. At each value of 2D-HSO, SDES is run and the profile of AvSD is generated at a 100-m interval. The most critical section with a minimum mean AvSD can also be identified. Same mean values of $h_{1}$ and $h_{2}$ used in step 3 are re-used in this step.

5. The horizontal curve is further superimposed with crest and sag curves with different rates of vertical curvature $(1 / K)$. The midpoint of the vertical curve in each case can be assumed to coincide with the midpoint of the horizontal curve.

6. For a certain combination of horizontal and vertical curves, the AvSD profile is again generated for each value of 2D-HSO using same mean values of $h_{1}$ and $h_{2}$. The minimum mean AvSD value for each combination is also determined.

7. The minimum 3D-HSO is then calculated using an added code to SDES. The code detects first the Cartesian coordinates of the tangency point between the sight line and side slope. Then, using the Cartesian coordinates of the midpoint between the driver eye and the object along the centerline of the inner lane, the horizontal projection of the lateral distance can be determined.

8. The difference in height $\left(h_{\mathrm{O}}\right)$ between the road surface at the mid-section and the point of tangency is also determined using the same Cartesian coordinates captured in step 6.

9. At a specific $R$, different speed distributions can be assumed. For each distribution, the $85^{\text {th }}$ percentile value can be taken as a design speed (Discetti and Dell'Acqua 2008). For simplicity, speed distributions can be selected such that their $85^{\text {th }}$ percentile values are multiples of $10 \mathrm{~km} / \mathrm{h}$.

10. Utilizing the speed distribution, along with those of $h_{1}, h_{2}, t$ and $a$, SDES can be run using $N$ iterations to predict both 3D AvSD and RqSD distributions. It should 
be mentioned that even though the change of $f$ can be included in the procedure, it was ignored in the presented application example since the $f$ distribution is different from a region to another.

11. At each set of values of $R, K$, and 2D-HSO, the $\mathrm{POH}$ profile is generated using each speed distribution and the maximum value is selected as a representative of such case.

12. Using all results from those steps above, a chart can be prepared to determine 3DHSO and $h_{\mathrm{O}}$ that satisfy a certain POH at a given design speed (V85). The chart also provides a useful tool to estimate 3D AvSD.

As an illustration example, the procedure listed above was applied to a horizontal curve with a 400-m radius combined with different vertical curves. A maximum grade of $10 \%$ was first assumed for both curve approaches and then the length of the vertical curve was changed giving a range of $K$ from 20 up to 100, for each vertical curve type (crest and sag). In addition, using the assumptions of the variables' distributions used earlier in CHAPTER 4 and CHAPTER 5, 2D-HSO, 3D-HSO, and 3D-AvSD were calculated using a mean $h_{1}$ and $h_{2}$ of $1.149 \mathrm{~m}$ and $0.726 \mathrm{~m}$, respectively.

In addition, the mean and standard deviation of the speed distributions were assumed to satisfy the relationship proposed by Andjus and Maletin (1998) (refer back to Equation 4-9). For a certain speed distribution, the design speed was reported in many instances in the literature to be close to the (V85) of such distribution. Utilizing the mean (V50) and standard deviation $(\sigma)$, and assuming a normal distribution for the speed, the $85^{\text {th }}$ percentile speed (V85) can be calculated as shown in Equation 7-1. 


$$
V 85=V 50+1.036 * \sigma
$$

Equation 7-1

\subsubsection{Design Aid and Application}

Using the procedures and assumptions listed in this section, a design aid was prepared as shown in Figure 7-4. The figure consists of two parts where the bottom part shows the relationship between the 3D-HSO on the horizontal axis and the minimum mean 3D AvSD on the left scale. The figure indicates that, at $R$ equal to $400 \mathrm{~m}$, the 3D AvSD may increase from 114 to $255 \mathrm{~m}$ when the 3D-HSO increases from 4 to $20 \mathrm{~m}$. The figure also shows a set of curves for the $\mathrm{POH}$ as presented on the right scale of the figure. The upper limit of 0.1 indicates that $10 \%$ of the drivers may suffer insufficient sight distance. The $\mathrm{POH}$ curves are described in terms of the design speed that ranges from 90 to $130 \mathrm{~km} / \mathrm{h}$. As explained earlier, a selected design speed would represent the $85^{\text {th }}$ percentile speed of a certain distribution. In general, the $\mathrm{POH}$ increases with the increase of speed and with the decrease in 3D-HSO.

On the other hand, the top part of Figure 7-4 is used to locate the height of the point of tangency at a given 3D-HSO. Such height depends on the vertical curve type and sharpness in addition to the values of $h_{1}$ and $h_{2}$. As shown in this figure, the case of a flat vertical alignment combined with the 400-m horizontal curve indicates a constant $h_{\mathrm{O}}$ of $0.94 \mathrm{~m}$ (average of 1.149 and $0.726 \mathrm{~m}$ ) over the entire range of 3D-HSO. On the other hand, the figure shows that the height increases or decreases when a sag or crest curve is combined with the horizontal curve, respectively. It can also be observed that, in the top part of the figure, the two lines representing crest curves with $K$ equal to 25 and 30 are truncated at 3D-HSO of 10.65 and $16.40 \mathrm{~m}$, respectively; indicating no more increase in 
AvSD with further increase in the corresponding 3D-HSO. The reason is that, at 3D-HSO higher than those values, the side slope is far enough such that the line of sight is obstructed by the road surface rather than the side slope. Such a scenario is not considered in the 2D methodology and charts presented in current design guides.

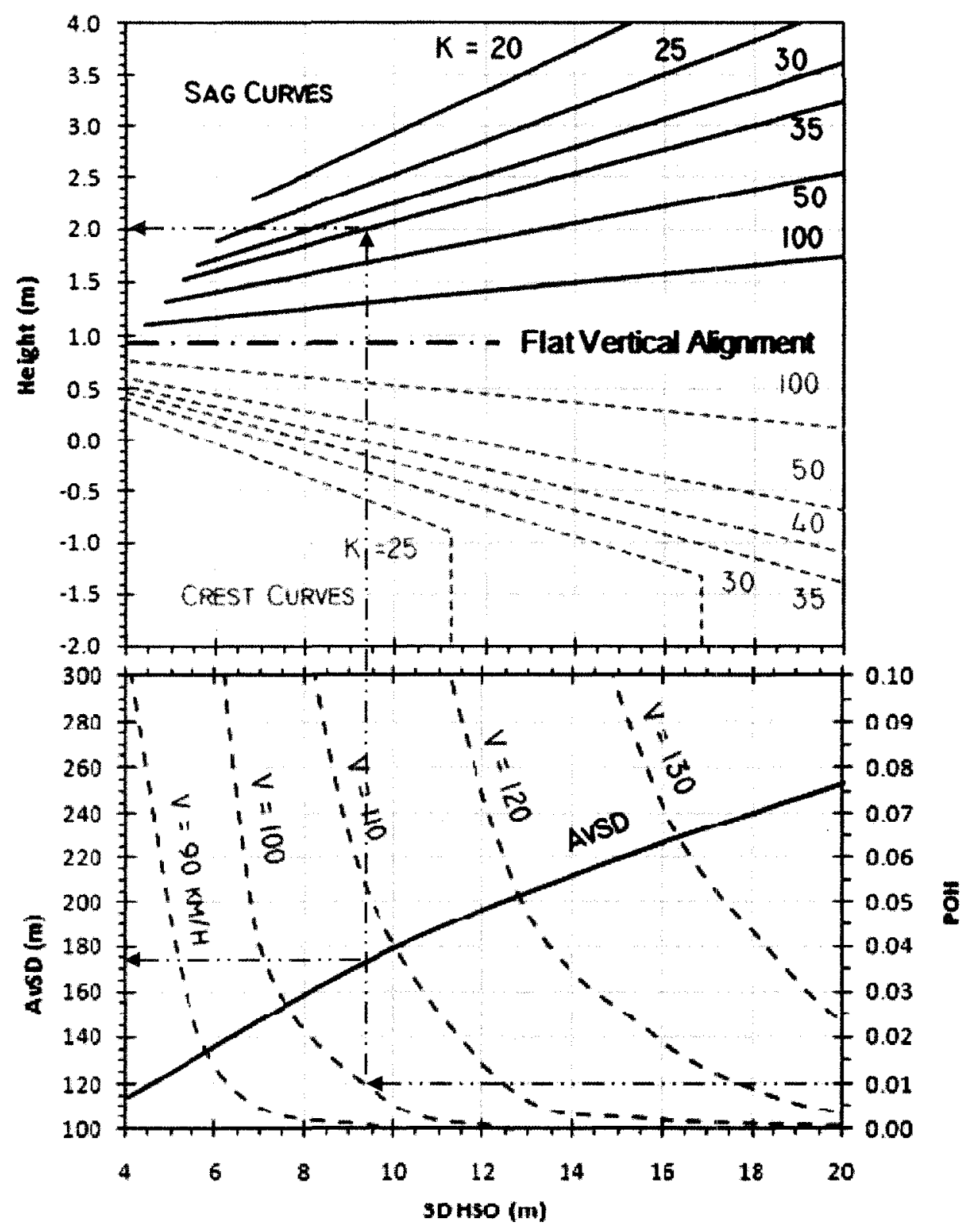

Figure 7-4: Reliability-Based Method for 3D-HSO Determination at $R=400 \mathrm{~m}$. 
The use of this chart should start by selecting a specific $\mathrm{POH}$ value that would match the road location and characteristics. For example, a road crossing an urban area with a relatively large number of access points or roads that experience frequent traffic queues may be designed at 1 or $2 \% \mathrm{POH}$. This low POH can also be selected for roads where falling debris or rocks are frequent. On the other hand, $\mathrm{POH}$ may be increased to 3 or $4 \%$ when roads are located in rural areas where incidents requiring drivers to perform an emergency stop are not as frequent. Using a certain POH, the 3D-HSO is first determined by selecting the design speed using one of the dotted curves in the bottom part of the graph. Two other values can be obtained using this 3D-HSO including (1) the minimum mean 3D AvSD expected on this section from the AvSD curve in the bottom part, and (2) the value of $h_{\mathrm{O}}$ at which this 3D-HSO has to be provided. This latter value is function of the type and $K$ of the vertical curve.

As an example, a $1 \% \mathrm{POH}$ on a $100-\mathrm{km} / \mathrm{h}$ road would require a 3D-HSO of $9.4 \mathrm{~m}$ along a horizontal curve with a radius of $400 \mathrm{~m}$. Assuming that the horizontal curve is superimposed with a sag curve with $K$ of 35 , the 9.4-m 3D-HSO is to be provided at or lower than $2.0 \mathrm{~m}$ from the road surface at the centerline of the inner lane. In addition, the figure indicates that the expected minimum mean 3D AvSD is $175 \mathrm{~m}$. According to the current design method in TAC (1999), 2D-HSO is calculated to be $13 \mathrm{~m}$ at $h_{\mathrm{O}}$ of $0.7 \mathrm{~m}$ (average of 1.05 and $0.35 \mathrm{~m}$ ). This might be uneconomic considering the 3D design methodology. Similar to this example, in case where a crest curve with $K$ of 35 is superimposed with the 400-m horizontal curve, same 3D AvSD of $175 \mathrm{~m}$ will also result if a 9.4-m 3D-HSO is provided at a height of -0.15 ; as per the top part of the figure. If the 
cross section has a non-vertical side slope, this would require more lateral space compared to the case of a sag curve.

\subsection{Hazard Evaluation of an Existing Design}

The developed chart is actually suitable for designing a new curve rather than evaluation of an existing curve. In this latter case, the designer would need to estimate the existing $\mathrm{POH}$ instead of determining 3D-HSO and $h_{\mathrm{O}}$. In this case, Figure 7-4 would not be easy to use as the point of tangency is hard to define on 3D alignments. Subsequently, the designer would not be able to determine the values of 3D-HSO and $h_{\mathrm{O}}$, where both values are required to estimate the expected $\mathrm{POH}$ on an existing curve. Therefore, except for the case of a horizontal curve overlapping with a constant-grade tangent, it would be difficult to predict where the line of sight is going to intersect with a side slope at a certain road section, unless actual measurement is taken. Given the impracticality of this approach, a tool is required to locate that point in terms of the design criteria of a road alignment and cross section.

One possible and simple way to predict the 3D-HSO is to express it in terms of the 2D-HSO at a given combination scenario of a horizontal and vertical curves. While the 3D-HSO and the location of the point of tangency are dependent on the vertical curvature, the 2D-HSO remains constant when either or both curve type and $K$ change; holding constant all other road configurations. Therefore, the 2D-HSO can first be calculated and then a tool could be used to correct it to a 3D-HSO given the type and sharpness of the vertical curve. However, a certain side slope has to be assumed for the validity of this approach. This is because the difference between 2D-HSO and 3D-HSO is 
expectedly zero for vertical-edge obstructions while the difference would increase using flatter side slopes.

The tool that is capable of doing such correction was developed in this section using the procedure listed before in Section 7.4.1 assuming a curve with a radius $400 \mathrm{~m}$ and side slope $2 \mathrm{H}: 1 \mathrm{~V}$, as an example. Different widths of the shoulder were then suggested and corresponding 2D-HSO were calculated. The horizontal curve was further superimposed with different vertical curves with different rates of vertical curvature ranging from 20 to 100 for each curve type. Having a certain curve combination, the 3DHSO was further calculated using SDES at each value of the shoulder width.

Following this HSO correction strategy, a chart was prepared as presented in Figure 7-5. The top part is used to correct the 2D-HSO calculated at a height of $0.94 \mathrm{~m}$; as an average of 1.149 and $0.726 \mathrm{~m}$ for $h_{1}$ and $h_{2}$, respectively. Using the calculated 2DHSO on the vertical axis, the 3D-HSO can be determined on the horizontal axis based on the type and $K$ of the vertical curve. For flat vertical alignment (the line of equality), the 2D-HSO is logically equal to the 3D-HSO. However, the difference starts to increase resulting in higher or lower values for the 3D-HSO when vertical curves are used. Furthermore, for the same vertical curve, the difference between the 2D-HSO and 3DHSO also increases with the increase of the 2D-HSO. 


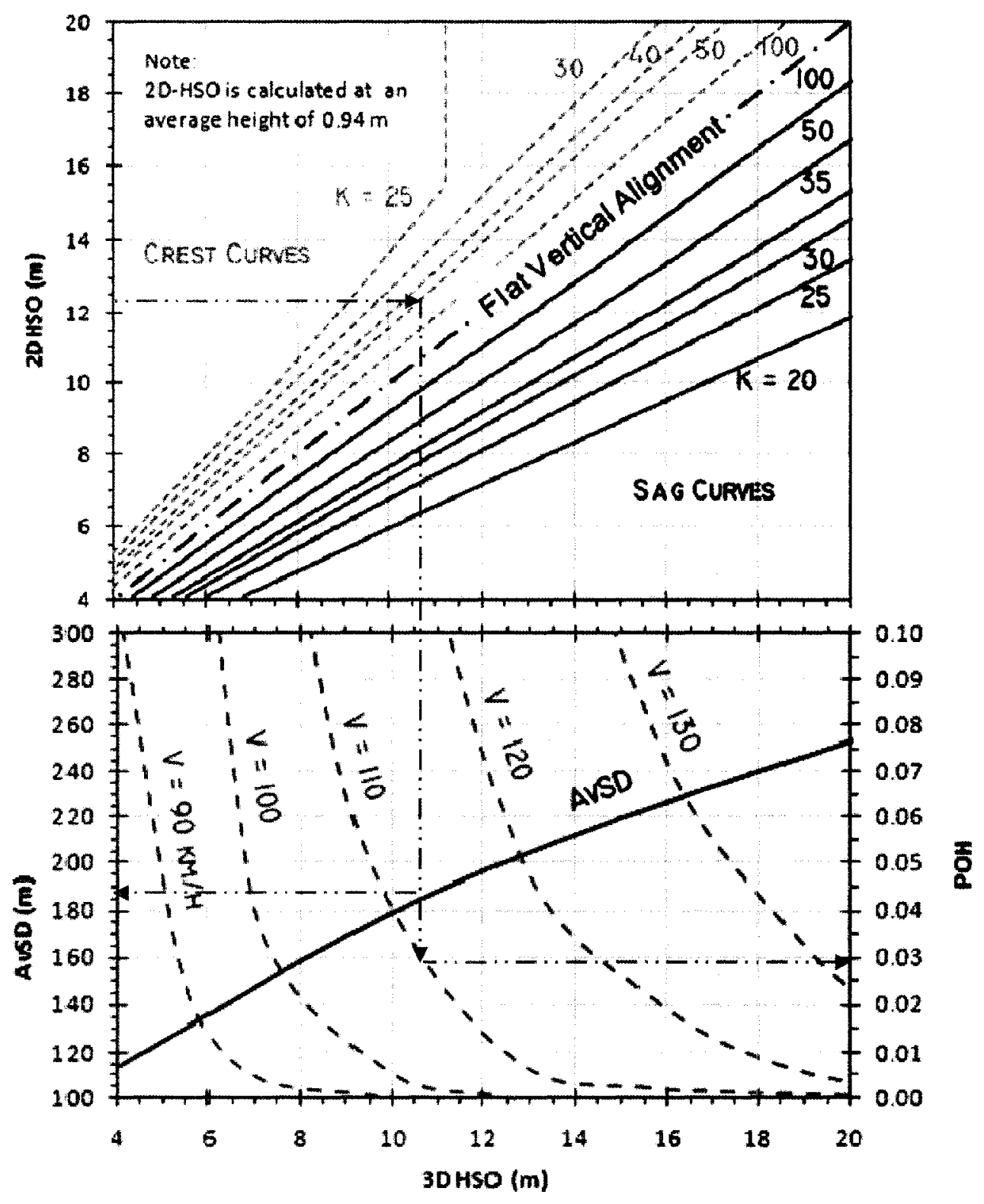

Figure 7-5: Hazard Evaluation of 3D Alignment at $R=400 \mathrm{~m}$ and $\mathrm{SS}=2 \mathrm{H}: 1 \mathrm{~V}$.

It should be mentioned, however, that the spread of the lines in this chart would change in a similar trend using a different value of the side slope. Moreover, the lines would all coincide with the line of equality when the side slope approaches zero. Once the 3D-HSO is determined, it is then possible to predict the 3D AvSD from the bottom part, which is the same part presented before in Figure 7-4. Moreover, the $\mathrm{POH}$ can be 
obtained using the right scale based on the speed. Several studies and models were reported in the literature for such speed estimation and can hence be used for this purpose. Most of these studies considered mainly the vehicle stability while little research handled the interrelationship between speed and AvSD. Further discussion is provided in the following section.

\subsection{Interrelationship between Speed and AvSD}

As mentioned before, the determination of an accurate speed distribution is important for a precise use of Figure 7-5. Generally, it is common that drivers adapt lower speeds on curves relative to straight road sections based on three main factors (1) vehicle stability, (2) alignment appearance, and (3) AvSD. For the third factor in particular, speed reduction can be interpreted from a reliability point of view such that, with the reduction of AvSD, drivers tend to shift back the RqSD distribution keeping an acceptable margin from the AvSD distribution. In other words, the overlap between the RqSD and AvSD, evaluated by the $\mathrm{POH}$, may not increase dramatically with the reduction of AvSD. Rather, drivers would exercise some caution and change the three main parameters affecting RqSD including speed, PR time, and deceleration rate so that the $\mathrm{POH}$ is kept at low levels. The reduction of PR time could be attained when drivers become more alerted while traversing a low-AvSD road segment. However, the increase in the overall hazard due to these changes/reductions should not be ignored even though POH might be quite low.

Based on the discussion above, the consideration of AvSD in speed estimation is recommended for better application of the developed methodology in this chapter. One of 
the unique studies that included the influence of AvSD on speed is the one by Discetti and Dell'Acqua (2008). The graph presented in that study was combined with a set of lines as suggested by Andjus and Maletin (1998). As shown in Figure 7-6, the right side of the graph may describe the $85^{\text {th }}$ percentile speed based on the horizontal curve radius, assuming that the AvSD is too large to affect speed. On the other hand, the left side indicates an expected speed reduction with the decrease of AvSD on sharp horizontal curves (i.e., $R=60$ to $250 \mathrm{~m}$ ). Although the maximum AvSD studied by Discetti and Dell'Acqua (2008) is $240 \mathrm{~m}$, extensions were made in Figure 7-6 to allow for a smooth transition filling the gap between each two lines in the middle part of the graph.

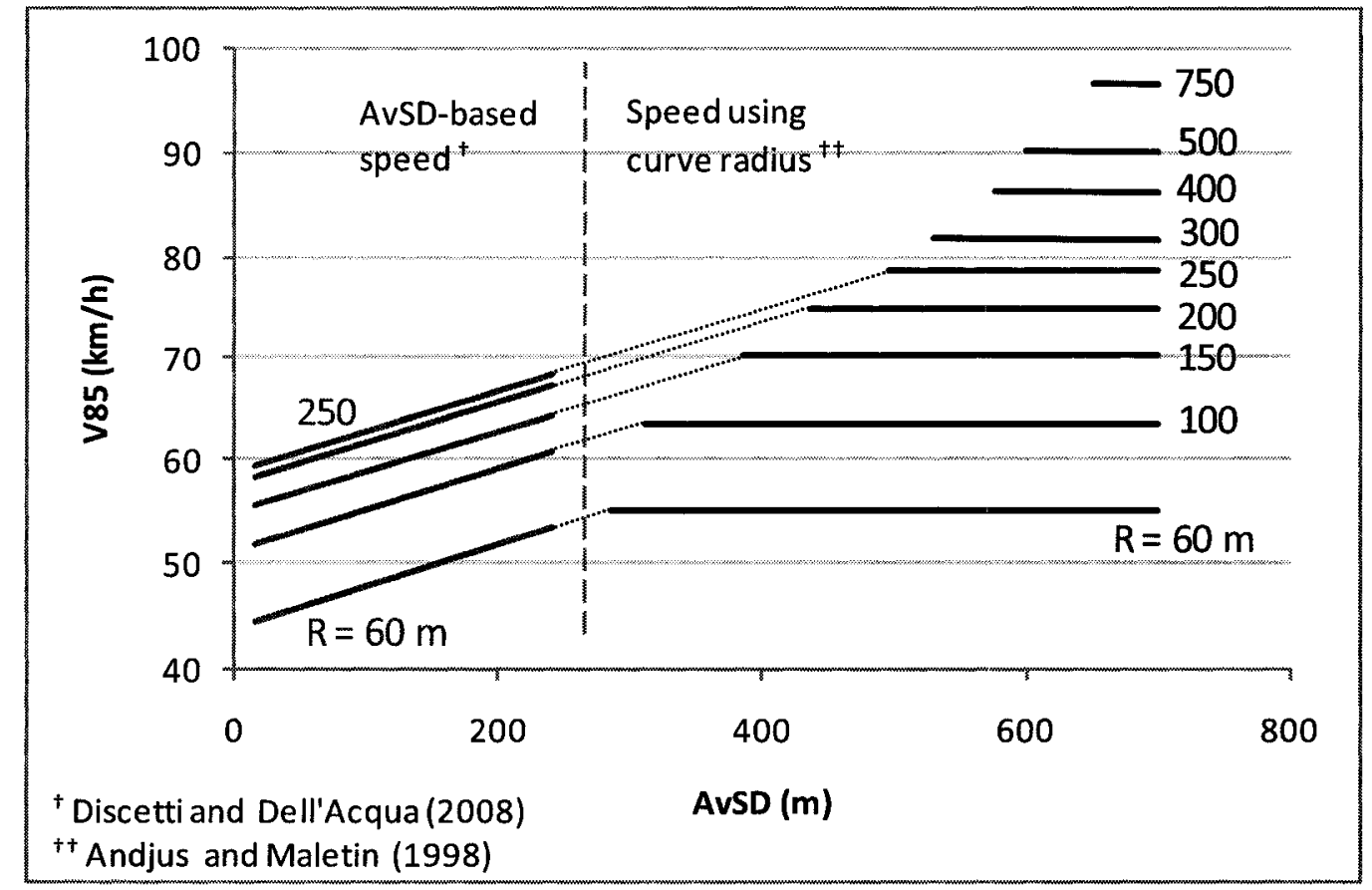

Figure 7-6: Influence of AvSD on Speed (Discetti and Dell'Acqua 2008 and Andjus and Maletin 1998). 
It should also be mentioned that the graph considers the radii limits reported in these two studies. Despite the fact that the outcome of these two studies would require validation before they can be applied in other districts, the graph highlights the influence of AvSD on speed and subsequently encourages further research in this track using a wider range of radius.

\subsection{Summary}

The discussion provided in this chapter highlighted the weaknesses of the current methodology of determining the lateral clearance on horizontal curve, as reported in TAC (1999) and AASHTO (2004). The key point in the criticism is the consideration of only the horizontal alignment along with the cross-section design. The current method is also based on the deterministic approach and might hence provide conservative and rigid designs. A new reliability-based methodology was therefore developed and suggested procedures were applied on a 400-m horizontal curve to locate lateral obstructions along a 3D alignment using a pre-determined $\mathrm{POH}$ value. The developed design aids in this chapter take into account the type and configuration of the vertical alignment and are valid for both design and evaluation purposes with the emphasis on considering the impact of AvSD on operating speed for accurate results. 


\section{CHAPTER 8}

\section{CONCLUSIONS AND RECOMMENDATIONS}

\subsection{Summary and Conclusions}

In current design guides, the dimensions of the highway elements are calculated using the deterministic approach. This approach depends on below average driver's capability and utilizes near-worst values for design parameters in an attempt to assure a safe driving environment for the majority of road users. However, the probability of having all parameters being simultaneously critical could be extremely small. Thus, a design following this approach could be uneconomic. In addition, the approach is rigid as it provides either minimum or maximum criteria of design elements such as curve radius, length, or grade. The approach is also lacking the ability to assess the consequences when such criteria cannot be met with the existence of certain constraints.

With the recognition of the drawbacks of the deterministic approach, researchers have been seeking potential usefulness of the reliability analysis as an alternative design approach. Conceptually, the probabilistic approach considers the variations of the design parameters instead of using a near-worst value for each one. A parameter's variation is expressed using a distribution with a mean and standard deviation. Using several reliability techniques, such as the analytical and simulation methods, a performance function can be expressed in terms of all parameters' distributions. A design can be then 
selected to satisfy a specific percentage of road users. This design approach is flexible enough to establish tradeoffs between cost and safety performance so that different alternatives can be assessed and optimum designs can be selected more confidently. The approach also allows more realistic consideration of the driver characteristics and gives an opportunity to evaluate a design on a scale of appropriateness instead of the misleading notion of right and wrong design. Throughout the literature, the reliability analysis has been successfully applied in numerous engineering branches such as structural and geotechnical. However, the progress in the transportation field is relatively slow.

The work presented in this research has successfully proposed a comprehensive framework for highway geometric design based on the reliability theory. The framework was further applied in this research on the sight distance design basis. A review of the literature showed that much of the data needed to apply such approach is currently available. Both required sight distance (RqSD), as a demand, and available sight distance (AvSD), as a supply, were evaluated using the analytical and simulation reliability methods. Numerical examples have been presented in this regard utilizing distributions of several design parameters such as speed, perception and reaction time, and driver eye and object heights, as reported in relevant studies in the literature.

In addition, a new approach was developed to calculate RqSD considering the continuous change of longitudinal grade on vertical curves and spline-grade segments. The approach also considers the possible loss of friction due to horizontal curvature. On the other hand, the 2D and 3D AvSD was considered in this research using two existing 
Dos-based computer programs based on the finite element techniques. The two programs have been re-coded using the Visual Basic.Net programming language and the new RqSD methodology was also programmed as a separate option. The new program; namely sight distance evaluation system (SDES), applies the simulation reliability technique and repeats the calculation of AvSD and RqSD for $N$ times along a road segment. In addition, using the RqSD and AvSD distributions at a given road section, the SDES calculates the percentage of cases where AvSD is less than RqSD representing the probability of hazard $(\mathrm{POH})$. Thus, a $\mathrm{POH}$ profile would describe the percentage of drivers who may suffer insufficient sight distance. Using POH profile, road sections with high hazard levels can be located and countermeasures can then be taken.

To show the possible contribution of the probabilistic approach to enhance the current design practice, a reliability-based approach was developed to locate lateral obstructions along 3D alignments based on a certain POH. Two design aids were further developed for direct use taking the advantages of SDES to efficiently calculate 3D AvSD and RqSD. The first design aid is useful for design purposes using a 400-m curve while the second one can be used to evaluate existing designs using $2 \mathrm{H}: 1 \mathrm{~V}$ side slope. Based on this summary and the findings of this research, the following can be concluded:

- The current highway geometric design methods rely solely on the deterministic approach. This approach is unable to accommodate the existing variation in the design parameters.

- The current methods to calculate RqSD in North American guides allows the use of a single value to account for the longitudinal grade. This could be suitable only on 
constant-grade road segments. However, the numerical examples presented in CHAPTER 3 indicated that a $40 \mathrm{~m}(24 \%)$ increase in RqSD could be expected when a vehicle traverses from a $+6 \%$ to $-6 \%$ grades, at $100-\mathrm{km} / \mathrm{h}$ speed.

- The use of a single value for the friction parameter in TAC (1999)'s SSD formula could not be acceptable if the braking distance is taking place, in whole or in part, on a curved section. The developed RqSD methodology again indicated that the reduction of the friction due to horizontal curvature may increase the difference between RqSD on a $+6 \%$ straight segment and $-6 \%$ curved one to $80 \mathrm{~m}(44 \%)$.

- Using numerical examples, the current methods to calculate RqSD in both AASHTO and TAC design guides were found producing very conservative designs. Using 100,000 trials in SDES indicated that a percentage as high as $98.5 \%$ of the driver population will be satisfied following AASHTO (2004)'s recommendations. In addition, TAC (1999) method is even more conservative that all drivers might be satisfied using its guidelines.

- The standard deviation of the RqSD distribution using 100,000 iterations in the simulation reliability technique $(17.85 \mathrm{~m})$ was found fairly close to the value $(16.80$ m) using the analytical formulas presented in CHAPTER 4. However, the analytical formulas are applicable only for constant-grade segments.

- The friction data collected in this research show that dry road surfaces, in both winter and summer seasons, might have a friction distribution with a mean of 0.907 and standard deviation of 0.045 . This distribution would indicate that the friction on dry surface may be sufficient for almost all drivers to decelerate at rates of their choice. In addition, the friction distribution may decrease on wet surfaces to a mean of 0.73 and 
standard deviation of 0.101 , which might be hazardous for speedy drivers with relatively high deceleration rates when they attempt to stop. Moreover, the friction distribution on snowy surfaces is expectedly the lowest with a mean of 0.26 and standard deviation of 0.05 . This distribution indicates insufficient friction for most of the drivers' deceleration rates.

- In AvSD calculations, the use of AASHTO recommendations $\left(h_{1}=1.08 \mathrm{~m}\right.$ and $h_{2}=$ $0.6 \mathrm{~m}$ ) may satisfy over $98.5 \%$ of the driver population based on recent recommendations of the parameters' distributions in the literature. Moreover, the TAC heights of 1.05 and $0.35 \mathrm{~m}$ for $h_{1}$ and $h_{2}$, respectively, could satisfy the entire population. This would re-stress the conservative nature of the current AvSD methods.

- The analytical reliability technique and developed formulas in CHAPTER 5 can be used to predict 2D AvSD distributions on isolated horizontal and vertical curves. The simulation reliability technique, however, remains the only applicable method on combined curves.

- The use of SDES indicated that the current 2D horizontal and vertical approaches in AvSD calculations may overestimate the actual 3D AvSD distribution by more than 7\%. In addition, the standard deviation of the AvSD distribution would increase in relative terms (from $0.46 \mathrm{~m}$ to $1.5 \mathrm{~m}$ ) if the line of sight is obstructed by the pavement surface (e.g., flat surface) rather than a $2 \mathrm{H}: 1 \mathrm{~V}$ side slope. Moreover, the standard deviation of AvSD will intuitively reach zero on vertical-edge lateral obstructions.

- Using two AvSD and RqSD distributions, there are different ways to assess the hazard associated with a certain design. The use of a probability of hazard ( $\mathrm{POH})$, 
however, may suite the nature of the geometric design. The $\mathrm{POH}$ reflects the percentage of those drivers who are operating at risk; with AvSD less than RqSD. Other reliability measures such as reliability indices $(\beta)$ might not be appropriate because no hazard due to insufficient sight distance could be assumed as long as all drivers can see ahead farther than they need to stop.

- Using speed data collected on 20 horizontal curves, a model was developed to predict the mean speed on two-lane roadways in terms of the curve radius $(R)$. The model is significant at $5 \%$ level of significance even though the coefficient of determination of 0.41 is low. In addition, no significant models were found to predict the standard deviation of speed in terms $R$.

- Using geometric data of Highway 61, Ontario, the POH profile was assessed along 6$\mathrm{km}$ segment of the road. Results indicated that most of the road sections had POH values less than $0.0005(0.05 \%)$. However, four sections had relatively high hazard levels between $3.4 \%$ and $7.5 \%$. Road sections with such high POH values could require either increasing AvSD or avoiding any traffic conflicts shortly after, such as signalized intersections.

- In CHAPTER 7, a reliability-based methodology was developed to design lateral clearance on 3D alignments considering a certain $\mathrm{POH}$. The methodology and design aids presented in this chapter indicate that the current design method using the deterministic approach produces very conservative designs besides its rigidity.

\subsection{Recommendations and Future Work}

Based on the work presented in this research, the following can be recommended: 
- In RqSD calculations, the change of longitudinal grade along braking distance and possible loss of friction on horizontal curves are ignored in the current design methods. For analysis on variable-grade segments and/or horizontal curves, the developed method to calculate an exact RqSD presented in CHAPTER 4 should be used simulating a vehicle stopping scenario.

- Using the probabilistic approach to estimate the RqSD distribution, both deceleration rate and friction coefficient have to be considered using the methodology presented in Section 4.4. The approach considers those cases where a road surface condition is unable to accommodate high deceleration rates.

- Accurate and detailed data for weather conditions are necessary for proper estimation of the RqSD distribution and POH. The data are useful to predict a friction distribution that considers different road surface conditions such as dry, wet, and snow.

- In AvSD calculations, the combined effect of the horizontal and vertical alignment could cause a considerable difference between the 2D and 3D AvSD values. Therefore, the three dimensional approach must be considered to avoid underestimating or overestimating the road supply.

- The data used in this research to model speed indicated no significant relationship between the curve radius and standard deviation. It also resulted in a model with a moderate coefficient of determination $\left(R^{2}=0.41\right)$ to predict the mean speed. Further studies in this regard are recommended for a better speed prediction. 
- It was stressed in CHAPTER 6 that a high value of POH is not necessarily leading to a more collision frequency. However, actual collision data could be useful to explicitly reveal the relationship between $\mathrm{POH}$ and safety performance.

- Throughout the literature, there are numerous models that were developed to predict speed in terms of the curve radius. However, very few studies addressed the impact of AvSD on speed. Thus, models could be required to estimate possible speed reductions on limited-AvSD road sections. 


\section{References}

AASHTO. 2004. A Policy on Geometric Design of Highways and Streets. American Association of State Highway and Transportation Officials. Washington, D.C.

McLean, J R and J. Morrall. 1998. Changes in Horizontal Alignment Design Standards in Australia And Canada. Transportation Research Board, $77^{\text {th }}$ Annual meeting.

AASHTO. 2001. A Policy on Geometric Design of Highways and Streets. American Association of State Highway and Transportation Officials. Washington, D.C.

AASHTO. 1984. A Policy on Geometric Design of Highways and Streets. American Association of State Highway and Transportation Officials. Washington, D.C.

AASHO. 1954. A Policy on Geometric Design of Rural Highways and Streets. American Association of State Highway Officials. Washington, D.C.

AASHO. 1940. A Policy on Sight Distance for Highways. American Association of State Highway Officials. Washington, D.C.

Andjus, Vojo and Mihailo Maletin. 1998. Speeds of Cars on Horizontal Curves. Transportation Research Record 1612: 42-47.

Ben-Akiva, Moshe, Moshe Hirshe, and Joseph Prashker. 1985. Probabilistic and Economic Factors in Highway Geometric Design. Transportation Science 19 (1): $38-57$.

Blasco, Ricardo D., Jose M. Prieto, and Jose M. Cornejo. 2003. Accident Probability after Accident Occurrence. Safety Science 41 (6): 481-501. 
Brydia, Robert and Martint Pietrucha. 1994. Analysis of Influence of PerceptionReaction on Case III Intersection Sight Distance. Transportation Research Record 1445: 189-197.

Cafiso, S. 2000. Experimental Survey of Safety Condition on Road Stretches with Alignment Inconsistencies. $2^{\text {nd }}$ International Symposium on Highway Geometric Design, Mainz, Germany, pp. 377-399.

Claret, Pablo Lardelli, Juan de Dios Luna del Castillo, Jose Juan Jimenez Moleon, Aurora Bueno Cavanillas, Miguel Garcia Martin, and Ramon Galvez Vargas. 2003. Age and Sex Differences in the Risk of Causing Vehicle Collisions in Spain, 1990 to 1999. Accident Analysis and Prevention 35 (2): 261-272.

Discetti, Paolo and Gianluca Dell'Acqua. 2008. Influence of Sight Distance on Driver Speed Behaviour for Low-Volume Roads. Transportation Research Board $87^{\text {th }}$ Annual Meeting.

Easa, Said M. 2000. Reliability Approach to Intersection Sight Distance Design. Transportation Research Record 1701: 42-52.

Easa, Said M. 1994. Reliability-Based Design of Sight Distance at Railroad Grade Crossings. Transportation Research-Part A: Policy and Practice 28 (1): 1-15.

Easa, Said M. and Yasser Hassan. 1997. Headlight Sight Distance on Separate Highway Alignment: A New Approach. Canadian Journal of Civil Engineering 24 (6): 1007-1018.

Easa, Said M., Essam Dabbour, and Muhammed Z. A. Ali. 2004. Three-Dimensional Model for Stop-Control Intersection Sight Distance. Journal of Transportation Engineering 130 (2): 261-270. 
Easa, Said M., A. O. Abd El Halim, and Yasser Hassan. 1996. Sight Distance Evaluation on Complex Highway Vertical Alignments. Canadian Journal of Civil Engineering 23 (3): 577-586.

Echaveguren, Tomás, Marcelo Bustos, and Hernán de Solminihac. 2005. Assessment of Horizontal Curves of an Existing Road Using Reliability Analysis Concepts. Canadian Journal of Transportation Engineering 32(6): 1030-1038.

El Khoury, John and Antine Hobeika. 2006. New Approach for Estimating the Passing Sight Distance Requirements. Transportation Research Board $85^{\text {th }}$ Annual Meeting.

Fambro, Daniel B., Kay Fitzpatrick, and Charles W Russell. 2000a. Operating Speed on Crest Vertical Curves with Limited Stopping Sight Distance. Transportation Research Record 1701: 25-31.

Fambro, Daniel B., Kay Fitzpatrick, and Rodger J Koppa. 2000b. New Stopping Sight Distance Model for Use in Highway Geometric Design. Transportation Research Record 1701: 1-8.

Fambro, Daniel B., Rodger J Koppa, Dale L. Picha, and Kay Fitzpatrick. 2000c. Driver Braking Performance in Stopping Sight Distance Situations. Transportation Research Record 1701: 9-16.

Fambro, Daniel B., J. Rodger Koppa, Dale L. Picha, and Kay Fitzpatrick. 1998. Driver Perception-Brake Response in Stopping Sight Distance Situations. Transportation Research Record 1628: 1-7. 
Fambro, D. B., K. Fitzpatrick, and R. J. Koppa. 1997. Determination of Stopping Sight Distances. National Cooperative Highway Research, Report 400. Transportation Research Board.

Fancher, P. S. 1986. Sight Distance Problem Related to Large Trucks. Transportation Research Record 1052:29-35.

Fitzpatrick, Kay, Torsten Lienau, and Danial B Fambro. 1998. Driver Eye and Vehicle Heights for Use in Geometric Design. Transportation Research Record 1612: 1-9.

Freedman, Mark, L. K. Staplin, and Lawrence E. Decina. 1985. Limited Sight Distance Warning for Vertical Curves. Public Roads 49 (2): 46-53.

Gattis, J. L., and J. Duncan. 1995. Geometric Design for Adequate Operational Preview of Road Ahead. Transportation Research Record 1500: 139-145.

General Motors Corporation and Delphi-Delco Electronic Systems. 2005. Automotive Collision Avoidance System Field Operational Test: Warning Cue Implementation Summary Report. Report DOT HS 809462, U.S. Department of Transportation. May 23, 2002.

Glennon, J. C. 1988. New and Improved Model of Passing Sight Distance on Two Lane Highways. Transportation Research Record 1195: 132-137.

Hall, Jerome W and Danial S Turner. 1998. Development and Adaption of Early AASHO Design Criteria. Transportation Research Record 1612: 26-33.

Harr, Milton. 1925. Reliability-Based Design in Civil Engineering. New York: McGrawHill Book Company.

Harwood, Douglas W. and John C. Glennon. 1989. Passing Sight Distance Design for Passenger Cars and Trucks. Transportation Research Record 1208: 59-69. 
Harwood, Douglas W., William D. Glauz, and John M. Mason. 1989. Stopping Sight Distance Design for Large Trucks. Transportation Research Record 1208: 36-46.

Hassan, Yasser. 2004. Three-Dimensional Approach for Roadway Alignment Design Incorporating Driver Perception. Advances in Transportation Studies, an international Journal, Section A 2.

Hassan, Yasser. 1996. Geometric design considerations of combined horizontal and vertical highway alignments. PhD Thesis, Carleton University, Ottawa, Canada.

Hassan, Y. and S. M. Easa. 2003. Effect of Vertical Alignment on Driver Perception of Horizontal Curves. Journal of Transportation Engineering 129 (4): 399-407.

Hassan, Yasser and Tarek Sayed. 2002. Effect of Driver and Road Characteristics on Required Review Sight Distance. Canadian Journal of Civil Engineering 29 (2): 276-288.

Hassan, Yasser and S. M. Easa. 2000. Technical Papers - Modeling of Required Preview Sight Distance. Journal of Transportation Engineering 126 (1): 13-20.

Hassan, Yasser and S. M. Easa. 1998a. Design of Sag Vertical Curves in ThreeDimensional Alignments. Journal of Transportation Engineering 124 (1): 52-58.

Hassan, Yasser and S. M. Easa. 1998b. Transportation Engineering - Sight Distance Red Zones on Combined Horizontal and Sag Vertical Curves. Canadian Journal of Civil Engineering 25 (4): 621-630.

Hassan, Y., T. Sayed, and S. Bidulka. 2002. Influence of Vertical Alignment on Horizontal Curve Perception - Phase II: Modeling Percieved Radius. Transportation Research Record 1796: 24- 34. 44782324 
Hassan, Yasser, S. M. Easa, and A. O. Abd El Halim. 1997. Modeling Headlight Sight Distance on Three-Dimensional Highway Alignments. Transportation Research Record 1579: 79-88.

Hassan, Yasser, S. M. Easa, and A. O. Abd El Halim. 1996. Analytical Model for Sight Distance Analysis on Three-Dimensional Highway Alignments. Transportation Research Record 1523: 1-10.

Hassan, Yasser, S. M. Easa, and A. O. Abd El Halim. 1995. Sight Distance on Horizontal Alignments with Continuous Lateral Obstructions. Transportation Research Record 1500: 31-42.

HSM. ANB25T Task Force on the Development of the Highway Safety Manual. Transportation Research Board. [Cited June 2, 2008] $<$ http://www.wsdot.wa.gov/partners/hsm/public/Home/Home.html $>$.

Jamil, Monther. 2005. Analysis of Friction Characteristics of Newly Resurfaced Roads in Ottawa. Master of Engineering, Carleton University, Ottawa, ON.

Jenkins, Jacqueline M. and Laurence R. Rilett. 2006. Modelling the Interaction Between Passenger Cars and Trucks. Report SWUTC/06/472700-00015-1, Texas Transportation Institute.

Lamm R., B. Psarianos, and T. Mailaender. 1999. Highway Design and Traffic Safety Engineering Handbook. Published by McGraw-Hill Companies.

Lovell, David J. 1999. Automated Calculation of Sight Distance from Horizontal Geometry. Journal of Transportation Engineering 125 (4): 297-304.

Lovell, David J, Jyh-Cherng Jong, and C. Peter. 2001. Improvements to Sight Distance Algorithm. Journal of Transportation Engineering 127 (4): 283-288. 
McGee, Hugh W. 1989. Reevaluation of the Usefulness and Application of Decision Sight Distance. Transportation Research Record 1208: 85-89.

Merritt, David R. 1988. Safe Speeds on Curves: A Historical Perspective of the Ball Bank Indicator. ITE Journal 85(9): 16-19.

Misaghi, P. and Yasser Hassan. 2005. Modeling Operating Speed and Speed Differential on Two-Lane Rural Roads. Journal of Transportation Engineering 131(6): 408417.

Navin, Francis P. D. and Jemay R. Zheng. 1998. Application of Reliability Theory to Highway Geometric Design. Transportation Research Board, $77^{\text {th }}$ Annual meeting.

Nehate, Girish and Malgorzata Rys. 2006. 3D Calculation of Stopping Sight Distance from GPS Data. Journal of Transportation Engineering 132 (9):691-698.

Neuman Timothy R. 1989. New Approach to Design for Stopping Sight Distance. Transportation Research Record 1208: 14-22.

Olson, P. L., D. E. Cleveland, P. S. Fancher. L. P. Kostyniuk, and L. W. Schneider. 1984. Parameters Affecting Stopping Sight Distance. National Cooperative Highway Research, Report 270. Transportation Research Board.

The Ontario Weather Page. [Cited July15, 2008] $<$ http://www.ontarioweather.com/analysis/ontarioarchives.asp $>$.

ORSAR, Ontario Road Safety Annual Report. [Cited February 15, 2008]. <http://www.mto.gov.on.ca/english/safety/orsar/orsar04/chp2_04.htm\#ref_2a>.

Polus, Abishai, Shmuel Borovsky, and Moshe Livneh. 1979. Limited Sight Distance Effect on Speed. Journal of Transportation Engineering 105 (5): 549-560. 
Reagan, Jerry A. 1994. The Interactive Highway Safety Design Model: Designing for Safety by Analyzing Road Geometrics. [Cited February 20, 2006]. $<$ http://www.tfhrc.gov/pubrds/summer94/p94su37.htm>.

Sigbjornsson, Ranger and Jonas Snaebjornsson. 1998. Probabilistic Assessment of Wind Related Accidents of Road Vehicles: A Reliability Approach. Journal of Wind Engineering and Industrial Aerodynamics 74-76: 1079-1090.

Smith, B.L. and Lamm, R. 1994. Coordination of Horizontal and Vertical Alignment with Regard to Highway Aesthetics. Transportation Research Record 1445: 73-85.

TAC. 1999. Geometric Design Guide for Canadian Roads. Transportation Association of Canada, Ottawa, Ontario.

Taiganidis, I. 1998. Aspects of Stopping-Sight Distance on Crest Vertical Curves. Journal of Transportation Engineering 124 (4): 335-342

Taiganidis, I. and George Kanellaidis. 2001. Technical Papers - Required Stopping Sight Distance on Crest Curves. Journal of Transportation Engineering 127 (4): 275282.

Taiganidis, I. and G. Kanellaidis. 1999. Approximate Perspective Design of Roads. Journal of Transportation Engineering 125 (4): 314-323.

Urbanik II, Thomas, Wanda Hinshaw, and Daniel B. Fambro. 1989. Safety Effects of Limited Sight Distance on Crest Vertical Curves. Transportation Research Record 1208: 23-35.

Wang, Yongji and M. P. Cartmell. 1998. Technical Papers - New Model for Passing Sight Distance on Two-Lane Highways. Journal of Transportation Engineering 124 (6): 536-545. 


\section{Additional Bibliography}

Al-Masaeid, H. R., Hamed, M., Aboul-Ela, M., and and Ghannam, A. G. 1994. Consistency of Horizontal Alignment for Different Vehicle Classes. Transportation Research Record 1500: 178-183.

Bidulka, Shaun, Tarek Sayed, and Yasser Hassan. 2002. Influence of Vertical Alignment on Horizontal Curve Perception; Phase I: Examining Curve Perception. Transportation Research Record 1796: 12-23.

Broyles, Robert W., Lutchmie Narine, S. Ross Clarke, and Daryl R. Baker. 2003. Factors Associated with the Likelihood of Injury Resulting from Collisions between FourWheel Drive Vehicles and Passenger Cars. Accident Analysis and Prevention 35 (5): 677-681.

Clarke, Nancy and Robert A Hagge. 2004. Evaluation of Traffic Safety Risk of Drivers Who Use Bioptic Telescopic Lenses. Transportation Research Record 1899: 127132.

Dabbour, Essam, Said M. Easa, and A. O. Abd El Halim. 2004. Radius Requirements for Reverse Horizontal Curves on Three-Dimensional Alignments. Journal of Transportation Engineering 130 (5): 610-620.

Donnell, Eric and John M Mason Jr. 2004. Predicting the Severity of Median-Related Crashes in Pennsylvania by Using Logistic Regression. Transportation Research Record 1897: 55-63. 
Durth, Walter and Markus Bernhard. 2000. Revised Design Parameters for Stopping Sight Distance. Proceeding of the 2nd International Symposium on Highway Geometric Design: 410-421.

Easa, Said M, Tim R. Strauss, Yasser Hassan, and Reginald R. Souleyrette. 2002. ThreeDimensional Transportation Analysis: Planning and Design. Journal of Transportation Engineering 128 (3): 250-258.

Easa, Said M. 1998. Three-Arc Vertical Curve for Constrained Highway Alignments. Journal of Transportation Engineering 124 (2): 163-171.

Easa, Said M. and Essam Dabbour. 2003. Design Requirements for Simple Horizontal Curves on Three-Dimensional Alignments. Canadian Journal of Civil Engineering 30: 1022-1033.

Fambro, Daniel B, John C. Collings, Probert Della Vedova, Joel P. Leisch, and John M. Mason. 2006. Geometric Design: Past, Present, and Future. [Cited August 30, 2008] <http://gulliver.trb.org/publications/millennium/00048.pdf>.

Fink, Kenneth L. and Raymond A. Krammes. 1995. Tangent Length and Sight Distance Effects on Accident Rates at Horizontal Curves on Rural Two-Lane Highways. Transportation Research Record 1500: 162-168.

Fitzpatrick, Key, Daniel B. Fambro, and Angela M. Stoddard. 2000. Safety Effects of Limited Stopping Sight Distance on Crest Vertical Curves. Transportation Research Record 1701: 17-24.

Fwa, T. F., W. T. Chan, and Y. P. Sim. 2002. Optimal Vertical Alignment Analysis for Highway Design. Journal of Transportation Engineering 128 (5): 395-402. 
Gibreel, G. M., A. M. Easa, Y. Hassan, and I. A. El-Dimeery. 1999. State of the Art of Highway Geometric Design Consistency. Journal of Transportation Engineering 125(4): 305-313.

Glennon, John C. 1989. Highway Sight Distance Design Issues: An Overview. Transportation Research Record 1208: 1-3.

Guevara, Felipe Ladron De, Simon p Washington, and Jutaek Oh. 2004. Forecasting Crashes at the Planning Level: Simultaneous Negative Binomial Crash Model Applied in Tucson, Arizona. Transportation Research Record 1897: 191-199.

Haas, Robert, Vaughan Inman, Andrew Dixson, and Davey Warren. 2004. Use of Intelligent Transportation System Data to Determine Driver Deceleration and Acceleration Behavior. Transportation Research Record 1899: 3-10.

Hall, J. W. and D. S. Turner. 1989. Stopping Sight Distance: Can We See Where We Now Stand? Transportation Research Record 1208: 4-13.

Hassan, Yasser and S. M. Easa. 1998. Design Considerations of Sight Distance Red Zones on Crest Curves. Journal of Transportation Engineering 124 (4): 343-352.

Hassan, Yasser and S. M. Easa. 2000. Modeling of Required Preview Sight Distance. Journal of Transportation Engineering 126 (1): 13-20.

Hassan, Yasser, S. M. Easa, and A.O.Abd El Halim. 1998. State-of-the-art of threedimensional highway geometric design. Canadian Journal of Civil Engineering 25: 500-511.

Hassan, Yasser, Said Easa, and A. O. Abd El Halim. 1998. Highway Alignment: ThreeDimensional Problem and Three-Dimensional Solution. Transportation Research Record 1612: 17-25. 
Huang, Ding-Wei and Yu-ping Wu. 2001. Car Accident on a Single-Lane Highway. Physical Review E. 63 (21): 022301-1 - 022301-4.

Jenkins, J. and L. R. Rilett. 2004. Application of Distributed Traffic Simulation for Passing Behavior Study. Transportation Research Record 1899: 11-18.

Jha, Manoj K. 2003. Criteria-Based Decision Support System for Selecting Highway Alignments. Journal of Transportation Engineering 129 (1): 33-41.

Kumara, S. S. P. and H. C. Chin. 2004. Study of Fatal Traffic Accidents in Asia Pacific Countries. Transportation Research Record 1897: 43-47.

Leisch, Joel P. 1989. Horizontal Sight Distance Considerations in Freeway and Interchange Reconstruction. Transportation Research Record 1208: 80-89.

McFadden, John and Lily Elefteriadou. 1998. Validation of Operating Speed-Based Design Consistency Models for Two-Lane Highways. Transportation Research Board, 77th Annual meeting.

Misaghi, P. and Y. Hassan. 2005. Modelling Operating Speed and Speed Differential on Two-Lane Rural Roads. Journal of Transportation Engineering 131 (6): 408-417. MUTCD. 1978. Manual on Uniform Traffic Control Devices for Streets and Highways. FHWA, U.S. Department of Transportation.

NCHRP. 2004. National Cooperative Highway Research Program. Development of a Highway Safety Manual. NCHRP Web Document 62 (Project 17-18[4]): Contractor's Final Report. [Cited April 2006]. $<$ http://gulliver.trb.org/publications/nchrp/nchrp_w62.pdf $>$. 
Polus, Abishai and Caroline Matter-Habib. 2004. New Consistency Model for Rural Highways and Its Relationship to Safety. Journal of Transportation Engineering 130 (3): 286-293.

Raeside, Robert and David White. 2004. Predicting Casualty Numbers in Great Britain. Transportation research Record 1897: 142-147.

Ratrout, Nedal T., Khalaf A. Al-Ofi, and Shoukat Iyaz. 2004. Validation and Improvement of Rear-End Conflict Prediction Model. Transportation Research Record 1897: 206-210.

Rudin-Brown, Christina M. 2004. Vehicle Height Affects Drivers' Speed Perception: Implications for Rollover Risk. Transportation Research Record 1899: 84-89.

Scoping, Project, Jake Kononov, and Bryan K. Allery. 2004. Explicit Consideration of Safety in Transportation Planning. Transportation Research Record 1897: 116125. 